\title{
Applications of ultra performance liquid chromatography (UPLC) and tandem mass spectrometry for the detection and quantification of cocaine, amphetamine, and opiate derivatives in human meconium
}

Joshua Adam Gunn

West Virginia University

Follow this and additional works at: https://researchrepository.wvu.edu/etd

\section{Recommended Citation}

Gunn, Joshua Adam, "Applications of ultra performance liquid chromatography (UPLC) and tandem mass spectrometry for the detection and quantification of cocaine, amphetamine, and opiate derivatives in human meconium" (2009). Graduate Theses, Dissertations, and Problem Reports. 4473.

https://researchrepository.wvu.edu/etd/4473

This Dissertation is protected by copyright and/or related rights. It has been brought to you by the The Research Repository @ WVU with permission from the rights-holder(s). You are free to use this Dissertation in any way that is permitted by the copyright and related rights legislation that applies to your use. For other uses you must obtain permission from the rights-holder(s) directly, unless additional rights are indicated by a Creative Commons license in the record and/ or on the work itself. This Dissertation has been accepted for inclusion in WVU Graduate Theses, Dissertations, and Problem Reports collection by an authorized administrator of The Research Repository @ WVU.

For more information, please contact researchrepository@mail.wvu.edu. 
Applications of Ultra Performance Liquid Chromatography (UPLC) and Tandem Mass Spectrometry for the Detection and Quantification of Cocaine, Amphetamine, and Opiate Derivatives in Human Meconium

By

Joshua Adam Gunn

Dissertation submitted to the College of Arts and Sciences at West Virginia University in partial fulfillment of the requirements for the degree of

Doctor of Philosophy

In

Chemistry

Approved by

Suzanne Bell, Ph.D., Chair

Fred King, Ph.D

Lisa Holland, Ph.D

Patrick Callery, Ph.D

Mary Wimmer, Ph.D

C. Eugene Bennett Department of Chemistry

Morgantown, West Virginia

2009

Keywords: Meconium, UPLC, Tandem mass spectrometry, Supported-liquid extraction, Electrospray, Desorption electrospray

Copyright 2009 Joshua A. Gunn 


\title{
Abstract \\ Applications of Ultra Performance Liquid Chromatography (UPLC) and Tandem Mass Spectrometry for the Detection and Quantification of Cocaine, Amphetamine, and Opiate Derivatives in Human Meconium
}

\author{
By Joshua A. Gunn
}

Development and validation of ultra performance liquid chromatography tandem mass spectrometry (UPLC-MS/MS) methodologies for the purpose of detecting and quantifying common drugs of abuse in human meconium specimens is described. Meconium is the first stool passed by a newborn infant. Meconium formation occurs over several months of gestation and subsequent toxicological analysis of the specimens can be useful for identifying drugs and other xenobiotics indicative of prenatal drug exposure.

Ultra performance liquid chromatography (UPLC) is an emerging analytical technique which draws upon the principles of chromatography to run separations at higher flow rates for increased speed, while simultaneously achieving superior resolution and sensitivity. Tandem mass spectrometry experiments were performed using a triple quadrupole mass spectrometer equipped with an electrospray ionization (ESI) source operating in positive ion mode.

Methodologies were developed and validated to detect and quantify amphetamine, methamphetamine, cocaine, benzoylecgonine, morphine, codeine, hydromorphone, and 6monoacetylmorphine (6-MAM) in authentic meconium specimens.

Analytes were extracted from the meconium matrix using either a mixed mode solid phase extraction (SPE), or a supported-liquid extraction (SLE) employing columns containing a modified form of diatomaceous earth. Data acquisition was performed using multiple reaction monitoring (MRM) and quantitation of each analyte was performed using a working standard 
calibration curve. The analytical methodologies were fully validated for the meconium matrix, where linearity, matrix equivalence, selectivity, accuracy, precision, sensitivity, stability, and recovery were evaluated.

Equivalence studies indicated that in all cases, certified drug free whole blood was a suitable matrix for the preparation of working calibration curves. Blank meconium specimens containing analytes at the LOQ, were spiked with various exogenous interferences and analyzed to assess the selectivity of the analytical method. All methodologies were deemed highly selective for the analyte of interest even in the presence of exogenous compounds commonly encountered in forensic specimens. Accuracy, precision, stability, and recovery were assessed at three different analyte concentrations corresponding to the LOQ, the ULOQ, and a concentration point midway between the two. Mean accuracies ranged from $94.6 \%$ to $99.6 \%$ over the three concentrations for the cocaine/benzoylecgonine methodology while the amphetamine and opiate assays also exhibited high accuracies with mean ranges of $93.6 \%$ to $98.4 \%$ and $93 \%$ to $99.6 \%$, respectively. Inter and intra batch precision data indicated enhanced method precision and reproducibility relative to existing techniques. Intra-batch $\mathrm{CV}$ values ranged from 1.6 to $11.8 \%$ for the cocaine/benzoylecgonine methodology while inter-batch $\mathrm{CV}$ values ranged from $3.9 \%$ to $6.2 \%$. Intra-batch $\mathrm{CV}$ values ranged from $0.7 \%$ to $8.5 \%$ for the amphetamine/methamphetamine methodology while inter-batch $\mathrm{CV}$ values ranged from $1.9 \%$ to $6.2 \%$. The opiate methodology was also highly precise with intra-batch $\mathrm{CV}$ values ranging from $1.2 \%$ to $10.7 \%$ while interbatch CV values ranged from $0.5 \%$ to $6.1 \%$ across the four analytes. While mean analyte recoveries ranged from $9.3 \%$ for benzoylecgonine to $76.3 \%$ for 6-monoacetylmorphine, the consistency and reproducibility of the extraction was acceptable for all analytes. Stability studies indicated that all analytes are stable in the meconium matrix when stored at $4^{\circ} \mathrm{C}$ and subjected to 
multiple freeze-thaw cycles over a 72 hour period. Limits of detection ranged from $250 \mathrm{pg} / \mathrm{mL}$ for methamphetamine, to $2.5 \mathrm{ng} / \mathrm{mL}$ for all four opiate analytes. Linear calibration for the cocaine/benzoylecgonine, amphetamine/methamphetamine, and opiate methodologies was achieved over the range of $10-250 \mathrm{ng} / \mathrm{mL}, 5-500 \mathrm{ng} / \mathrm{mL}$, and $10-500 \mathrm{ng} / \mathrm{mL}$, respectively.

Recent technological advances made in the field of particle chemistry mean that liquid chromatographic separations can be performed at higher flow rates for increased speed without sacrificing resolution or sensitivity. The increased speed, resolution, sensitivity, and separation efficiency afforded by UPLC combined with the inherent selectivity and sensitivity of the tandem mass spectrometer allowed for the accurate quantitation of all 8 analytes in the meconium matrix in a time and cost effective manner. Development and validation of such analytical methodologies will prove beneficial for the identification of prenatal substance abuse which is an ongoing concern across socioeconomic lines. 


\section{Dedication}

To my loving parents (Kathy and Brian), my brother (Luke), my sister (Sarah), my girlfriend (Ashley), and the many members of the Gunn and Jess families, I dedicate this dissertation.

In loving memory of Morris (Digger) \& Eileen Jess and Charlie \& Jean Gunn. 


\section{Acknowledgements}

It is difficult to describe the eternal gratitude I have for my family members whose ongoing love, support, and belief has been the biggest inspiration in my life. To my mother Kathy, my father Brian, my brother Luke, and my sister Sarah, thank you for constantly supporting all of my ventures in life, and believing in me no matter what the task at hand. Your love and support has provided me with the motivation and confidence to continue learning and achieving in times when the distance between us simply seemed too great. You have all played an integral role in my life and your unconditional love, support and willingness to chat at any time of the day or night will never be forgotten nor taken for granted.

I would like to thank my $\mathrm{PhD}$ supervisor Dr Suzanne Bell not only for her support, guidance, and unconditional assistance throughout the course of my doctoral degree, but also for her willingness to assist me in becoming a part of the graduate program at WVU.

Thank you to the individual members of my PhD committee for their ongoing academic assistance throughout the course of my PhD. To Dr Patrick Callery, Dr Lisa Holland, Dr Fred King, and Dr Mary Wimmer, thank you for supporting me not only throughout the final stages of my $\mathrm{PhD}$ but also during the initial coursework, cumulative exams, academic seminars, and oral proposals associated with the PhD program at WVU. I have thoroughly enjoyed my time at WVU and wish to thank each and every one of you for making it the wonderful experience that is has been.

It would be impossible to appropriately thank the individual members of the Bell research group for all that they have done for me during my time at WVU. I am eternally grateful to each and every one of you for continuously picking me up from the airport, driving me to and from 
class, translating American terminology for me, and putting a roof over my head. You are all very special to me and I look forward to working with you in the future.

Thank you to Joe Kennedy and Dr Justin Wiseman of Prosolia for their assistance with all DESI experiments. Your assistance and expertise are greatly appreciated and I look forward to a continued collaboration with your company.

Finally I would like to sincerely thank Dr Michael Evans and Dr Andrea Terrell for allowing me to complete my doctoral research at AIT Laboratories. I have thoroughly enjoyed my time at AIT and am excited at the prospect of remaining a member of the AIT team. 


\section{Table of Contents}

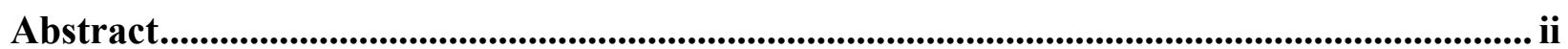

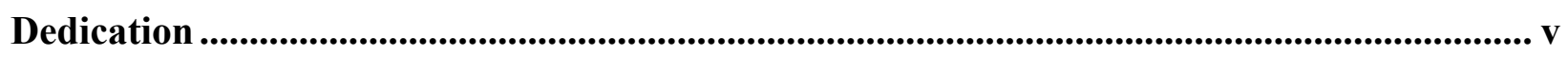

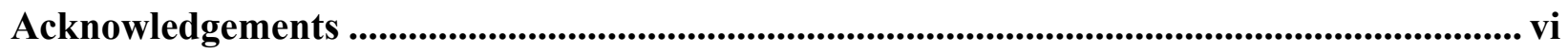

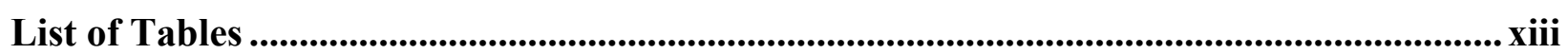

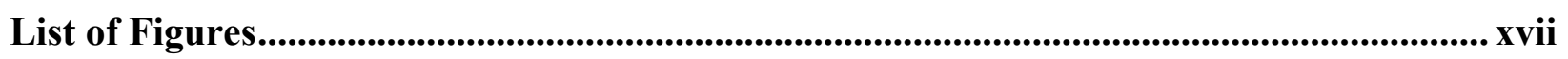

List of Abbreviations, Symbols/Nomenclature.................................................................. xxii

Chapter 1: Introduction to Prenatal Substance Abuse and Its Clinical and Forensic

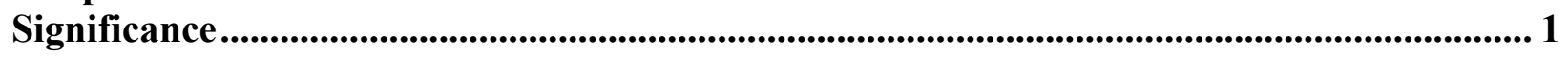

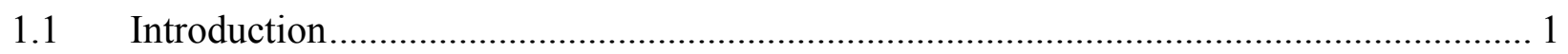

1.2 Drug Transfer in the Maternal-Fetal Complex .................................................... 4

1.3 Traditional Testing Procedures ............................................................................ 6

1.4 History of Meconium Analysis ............................................................................... 10

1.4.1 Instrumental Methodologies Employed for the Analysis of Meconium ............. 17

Chapter 2: Introduction to the Laboratory Analysis of Toxicological Specimens ............... 19

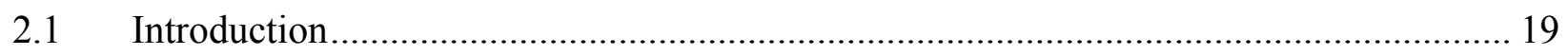

2.2 Sample Preparation Techniques.............................................................................. 23

2.2.1 Liquid - Liquid Extraction (LLE) ..................................................... 24

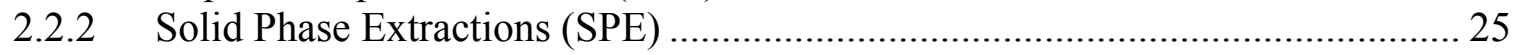

2.3 Chromatographic Separations in Forensic Toxicology.......................................... 27

2.4 Ultra Performance Liquid Chromatography (UPLC) ............................................ 29

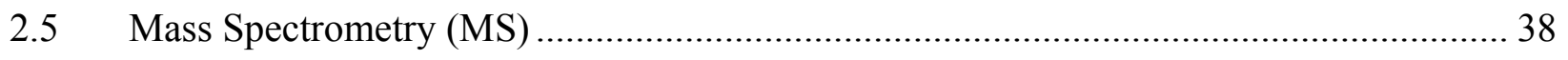

2.6 The Rise of Liquid Chromatography - Mass Spectrometry (LC/MS) ......................... 41

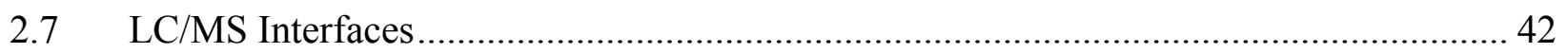

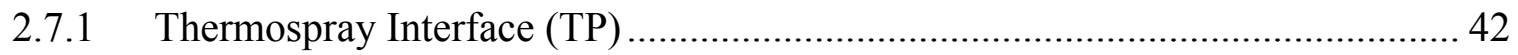

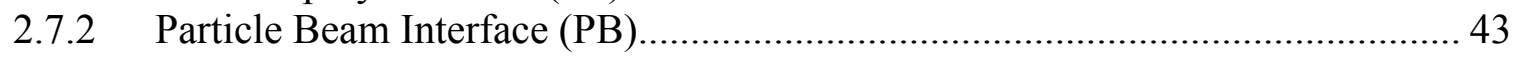


2.7.3 Fast Atom Bombardment Interface (FAB) .................................................... 44

2.7.4 Atmospheric Pressure Ionization (API) Techniques ............................................ 44

2.7.5 Atmospheric Pressure Chemical Ionization (APCI) …………………............. 47

2.7.6 Electrospray Ionization (ESI) .................................................................... 49

2.8 LC/MS/MS and its Applications in Forensic Toxicology …………………………........ 54

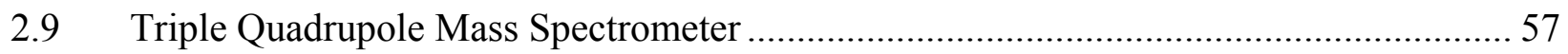

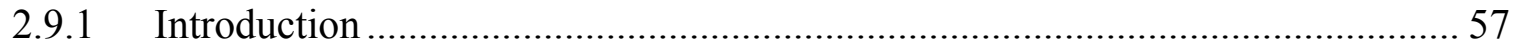

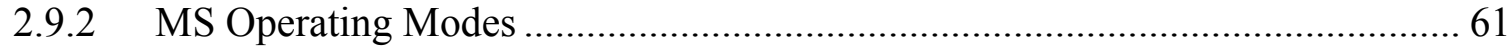

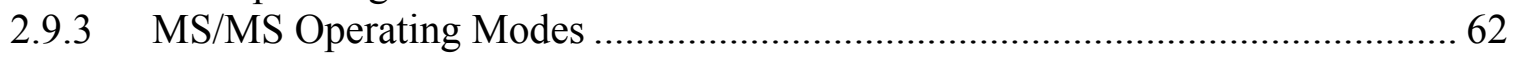

2.10 Mass Spectral Criteria for a Positive Identification......................................................... 67

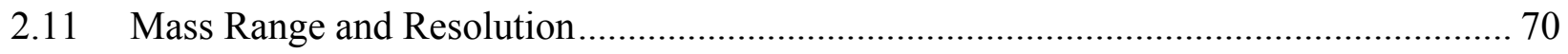

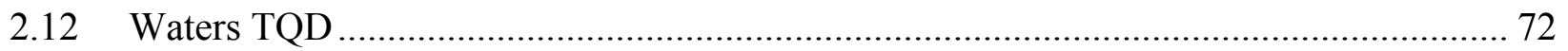

2.13 Application of Tandem Mass Spectrometry in Forensic Toxicology ................................ 74

Chapter 3: Analytical Method Validation ........................................................................................ 84

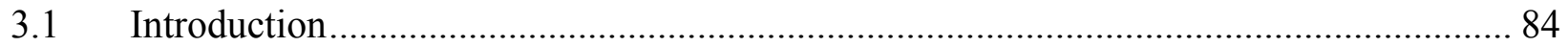

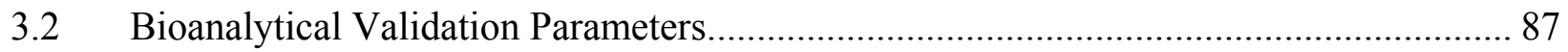

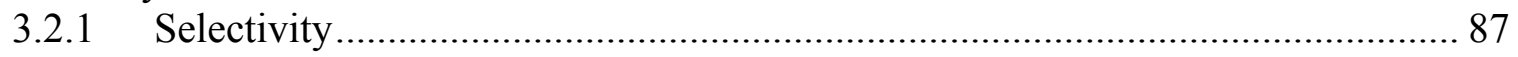

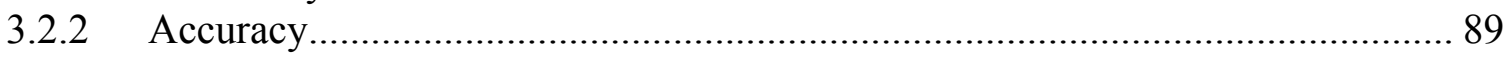

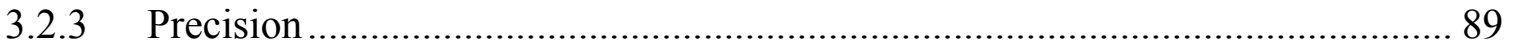

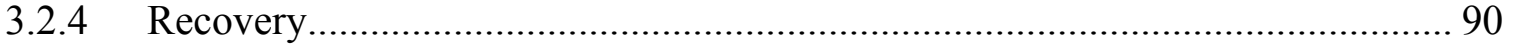

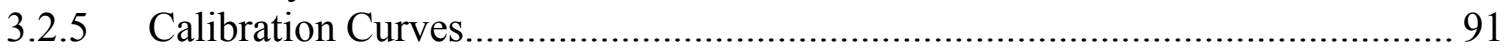

3.2.6 Stability Studies........................................................................................... 91

3.2.7 Limit of Detection (LOD) and Lower Limit of Quantitation (LLOQ) Studies... 92

Chapter 4: Introduction and Overview to the Development and Validation of UPLCMS/MS Methodologies for the Quantitative Determination of Cocaine, Amphetamine, and Opiate Derivatives in Human Meconium. ........................................................................................ 96

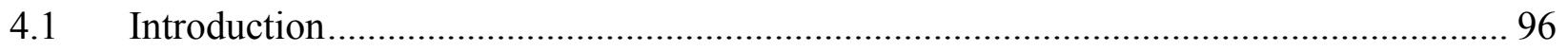

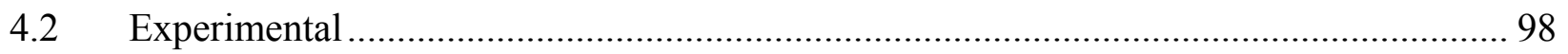

4.2.1 Development and Optimization of MS/MS Parameters...................................... 98

4.2.2 Development of UPLC Separation Conditions …………………...................... 100

4.2.3 Considerations for UPLC-MS/MS Method Development ................................. 100

4.3 Experimental Design for Method Validation................................................................. 105

4.3.1 Equivalence Studies for Calibration Curves ……………………………….... 107

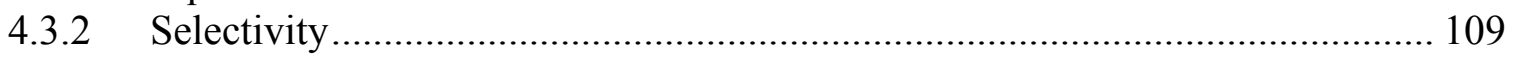




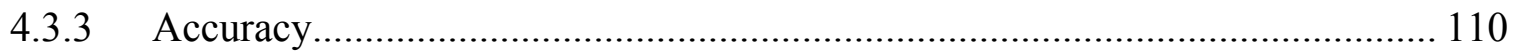

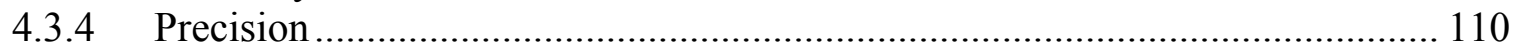

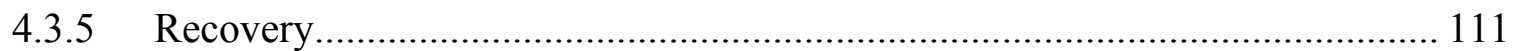

4.3.6 Limit of Detection (LOD) and Limit of Quantitation (LOQ) ........................... 111

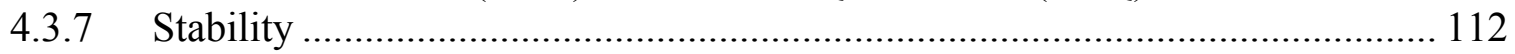

4.3.8 Data Analysis ........................................................................................ 113

Chapter 5: Detection and Quantification of Cocaine and Benzoylecgonine in Meconium Using Solid Phase Extraction and UPLC-MS/MS ................................................................... 114

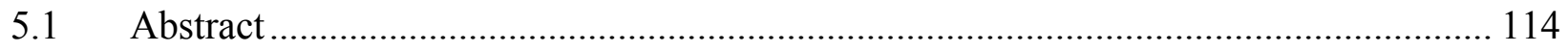

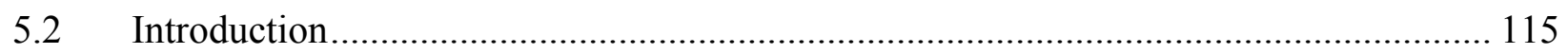

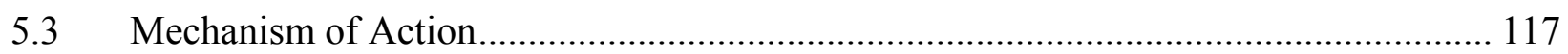

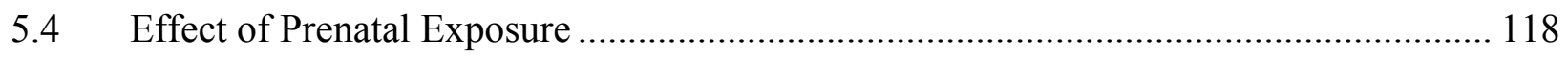

5.5 History of Cocaine and Benzoylecgonine Detection in Meconium Specimens. ............ 118

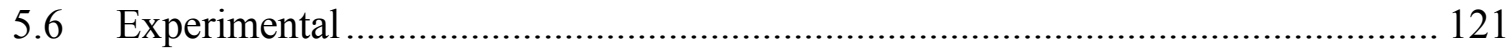

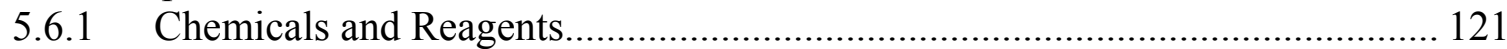

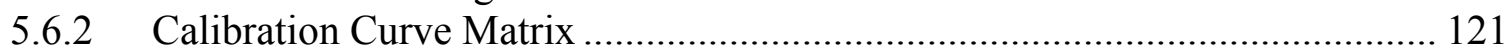

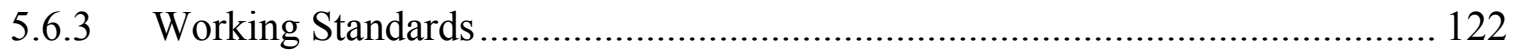

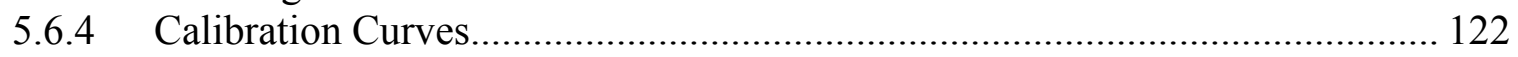

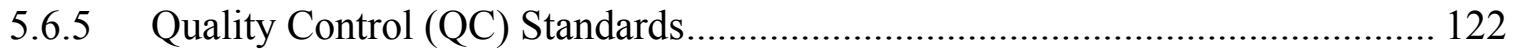

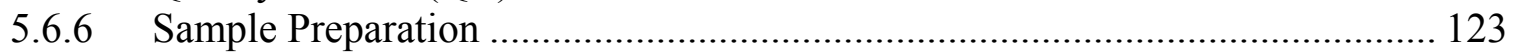

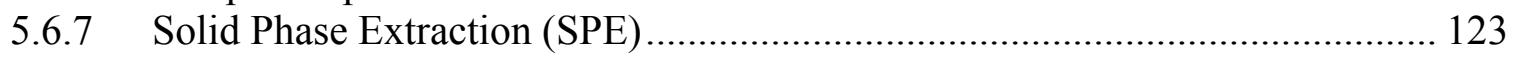

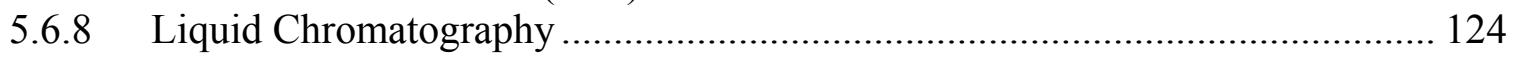

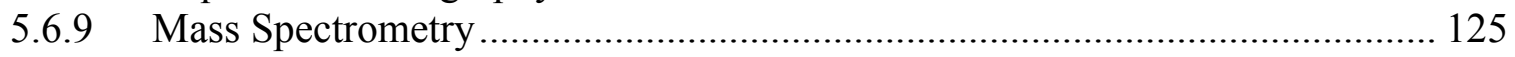

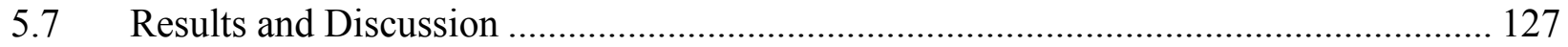

5.7.2 Method Validation................................................................................. 127

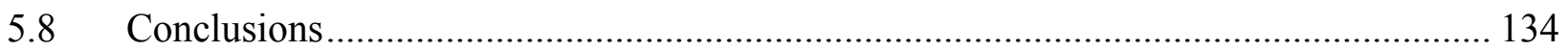

Chapter 6: Detection and Quantification of Codeine, Morphine, Hydromorphone, and 6Monoacetylmorphine (6-MAM) in Meconium using ISOLUTE HM-N Supported-Liquid Extraction Columns and UPLC-MS/MS ...................................................................................... 135

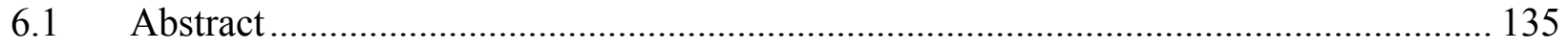

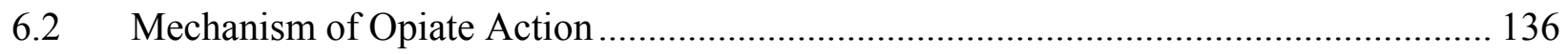

6.3 Introduction to Commonly Abused Opiates ……………............................................... 139

6.4 History of Opiate Detection in Meconium.................................................................... 147 


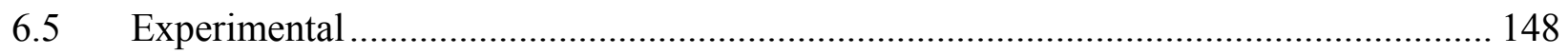

6.5.2 Calibration Curve Matrix .......................................................................... 148

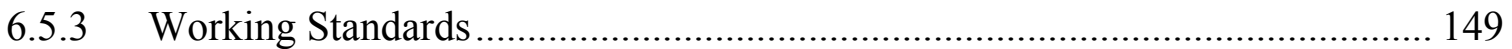

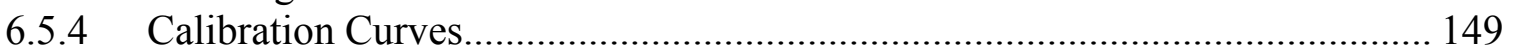

6.5.5 Quality Control (QC) Standards................................................................... 150

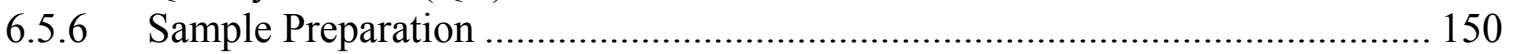

6.5.7 Liquid Chromatography ........................................................................... 151

6.5.8 Mass Spectrometry ................................................................................ 152

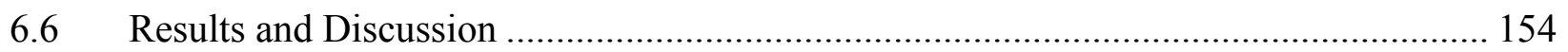

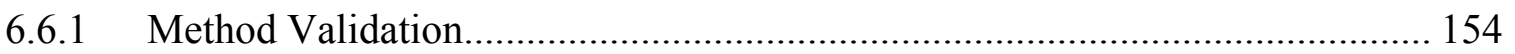

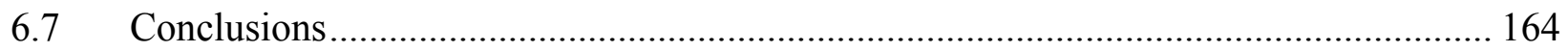

Chapter 7: Simultaneous Quantification of Amphetamine and Methamphetamine in Meconium Using ISOLUTE ${ }^{\circledR}$ HM-N Supported Liquid Extraction Columns and UPLC-

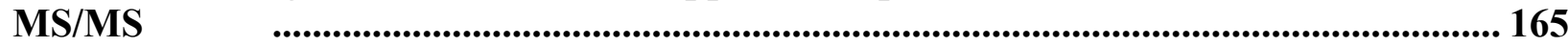

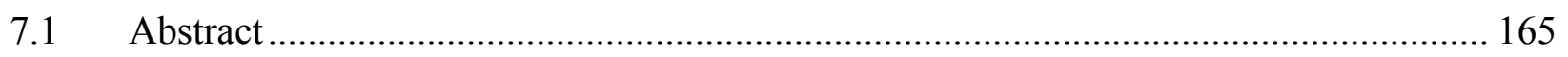

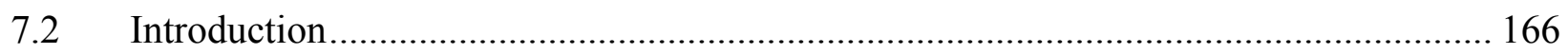

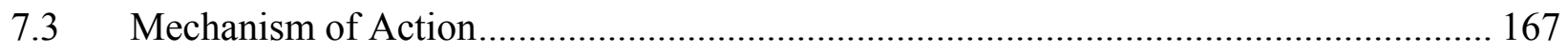

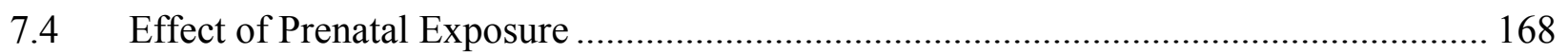

7.5 History of Amphetamine Analysis in Meconium (Literature Review) ........................... 168

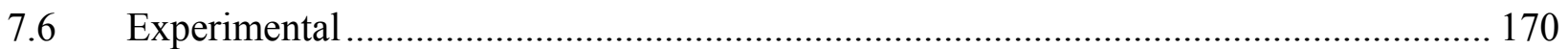

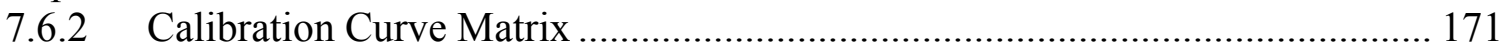

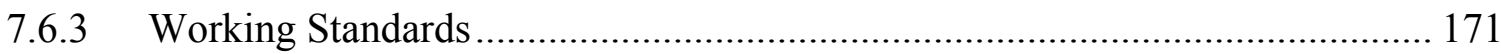

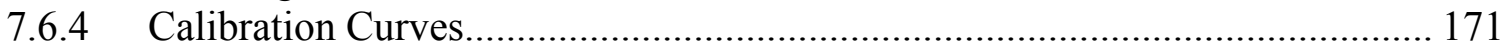

7.6.5 Quality Control (QC) Standards................................................................ 172

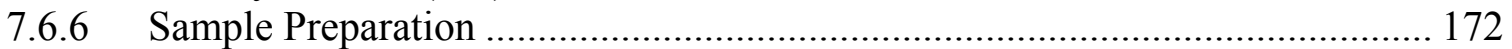

7.6.7 Liquid Chromatography ............................................................................. 173

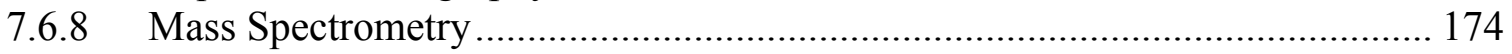

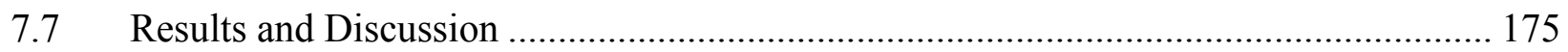

7.7.1 Chromatographic Separation of Amphetamine and Methamphetamine ........... 176

7.7.2 Method Validation................................................................................... 182

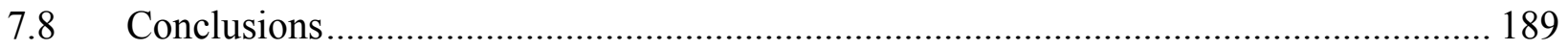

Chapter 8: Investigations into the Application of Desorption Electrospray Ionization (DESI) for the Rapid Screening of Meconium Specimens ............................................................ 191

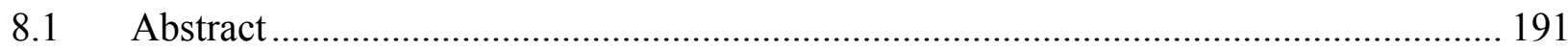




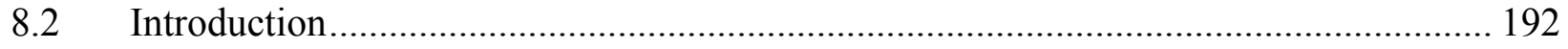

8.2.1 Desorption Electrospray Ionization (DESI) ………........................................ 192

8.2.2 Matrix Assisted Laser Desorption Ionization (MALDI) ................................... 193

8.2.3 History and Background of Meconium Screening............................................ 197

8.2.4 Mass Spectrometry as a Screening Tool ......................................................... 207

8.2.5 Principles of Desorption Electrospray Ionization (DESI) ............................... 215

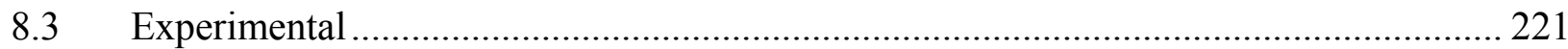

8.3.1 Chemicals and Reagents............................................................................ 221

8.3.2 Electrospray Solvent Preparation.................................................................. 222

8.3.3 DESI Source and Mass Spectrometry Conditions............................................ 222

8.3.4 Sample Preparation …………………………...................................... 222

8.3.5 DESI Analysis of Standards ..................................................................... 222

8.3.6 Direct Analysis of Spiked Meconium Specimens.............................................. 225

8.3.7 Sample Preparation ............................................................................... 225

8.3.8 Preliminary Results ................................................................................. 226

8.3.9 Meconium Sample Preparation Method \#1 ……………................................... 231

8.3.10 Meconium Sample Preparation Method \#2 …………………………………... 235

8.4 Qualitative Determination of Amphetamine, Methamphetamine, Cocaine, and

Benzoylecgonine in Authentic Meconium Specimens using DESI-MS/MS.............................. 250

8.4.1 Experimental Design ............................................................................. 250

8.4.2 Sample Preparation for DESI-MS/MS Screening Experiments........................ 252

8.4.3 Preparation of Positive Quality Control Specimens........................................... 252

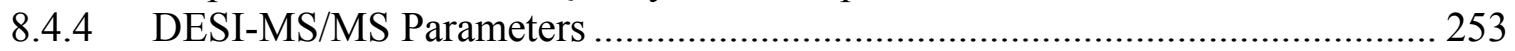

8.4.5 DESI-MS/MS Screening Results ............................................................... 253

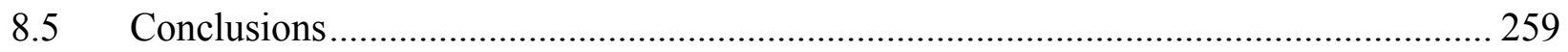

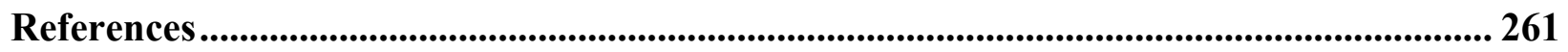




\section{List of Tables}

Table 1: Common analytes detected during 'drugs of abuse' screening and confirmation analysis.

Table 2: Components of the Van deemter equation and their relationship to column and analyte properties

Table 3: MS operating modes using the triple quadrupole mass spectrometer. 62

Table 4: MS/MS operating modes of the triple quadrupole mass spectrometer. 62

Table 5: Select system specifications of the Waters triple quadrupole detector (TQD). 74

Table 6: Overview of bioanalytical method validation terms and definitions. 87

Table 7: Overview of three UPLC-MS/MS methodologies developed and validated for the purpose of detecting and quantifying several cocaine, amphetamine, and opiate derivatives in human meconium. 97

Table 8: Concentrations of standards used in all validation experiments. Concentrations of standards spanned the calibration range of each method. 107

Table 9: Concentrations of all LQC and HQC standards used in preliminary matrix equivalence studies. 108

Table 10: Chemical structures, molecular weights, molecular formulas, and pKa's of cocaine and benzoylecgonine. 117

Table 11: Preparation of cocaine and benzoylecgonine calibration curves. 122

Table 12: MS/MS parameters used for each analyte and deuterated internal standard. 126

Table 13: Cocaine equivalence studies using quantitative values obtained from calibration curves constructed in meconium (MC) and blood (BC). 128

Table 14: Benzoylecgonine equivalence studies using quantitative values obtained from calibration curves constructed in meconium (MC) and blood (BC)...... 128

Table 15: Exogenous compounds included in selectivity quality control standards.

Table 16: Cocaine selectivity studies using blank meconium from five different sources and blank meconium specimens spiked with various exogenous interferences.

Table 17: Benzoylecgonine selectivity studies using blank meconium from five different sources and blank meconium specimens spiked with various exogenous interferences. 129

Table 18: Cocaine accuracy studies. 130 
Table 19: Benzoylecgonine accuracy studies. 130

Table 20: Intra- and inter-batch precision studies for cocaine. 131

Table 21: Intra- and inter-batch precision studies for benzoylecgonine (BE). 131

Table 22: Receptor activity for selected opiates and synthetic derivatives. 138

Table 23: Chemical structures, molecular weights, molecular formulas, and pKa's of morphine, codeine, hydromorphone, and 6-monoacetylmorphine. 146

Table 24: Preparation of codeine, morphine, hydromorphone, and 6-monacetylmorphine calibration curves.

Table 25: MS/MS parameters used for each analyte and their deuterated internal standards. ... 153

Table 26: Codeine equivalence studies using quantitative values obtained from calibration curves constructed in meconium (MC) and blood (BC). 155

Table 27: Morphine equivalence studies using quantitative values obtained from calibration curves constructed in meconium (MC) and blood (BC). 155

Table 28: Hydromorphone equivalence studies using quantitative values obtained from calibration curves constructed in meconium (MC) and blood (BC). 155

Table 29: 6-monoacetylmorphine (6-MAM) equivalence studies using quantitative values obtained from calibration curves constructed in meconium (MC) and blood (BC).................. 156

Table 30: Exogenous compounds included in selectivity quality control standards. .................. 156

Table 31: Codeine selectivity studies using blank meconium from five different sources and blank meconium specimens spiked with various exogenous interferences. 157

Table 32: Morphine selectivity studies using blank meconium from five different sources and blank meconium specimens spiked with various exogenous interferences. 157

Table 33: Hydromorphone selectivity studies using blank meconium from five different sources and blank meconium specimens spiked with various exogenous interferences. 157

Table 34: 6-monoacetylmorphine selectivity studies using blank meconium from five different sources and blank meconium specimens spiked with various exogenous interferences. 157

Table 35: Codeine accuracy over three concentrations spanning the calibration range............. 158

Table 36: Morphine accuracy over three concentrations spanning the calibration range............ 158

Table 37: Hydromorphone accuracy over three concentrations spanning the calibration range. 158 
Table 38: 6-monoacetylmorphine accuracy over three concentrations spanning the calibration range.

Table 39: Intra- and inter-batch precision for codeine............................................................ 160

Table 40: Intra- and inter-batch precision for morphine........................................................... 160

Table 41: Intra- and inter-batch precision for hydromorphone. ............................................... 160

Table 42: Intra- and inter-batch precision for 6-monoacetylmorphine (6-MAM) ....................... 160

Table 43: Chemical structures, molecular weights, molecular formulas and pKa's of

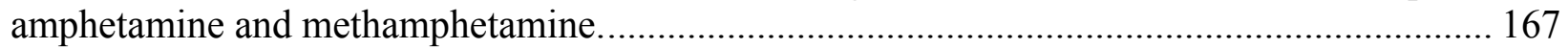

Table 44: Preparation of amphetamine and methamphetamine calibration curves. ................... 172

Table 45: MS/MS parameters used for each analyte and deuterated internal standard.............. 174

Table 46: Amphetamine equivalence studies using quantitative values obtained from calibration

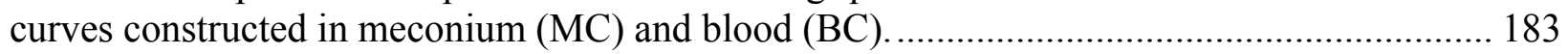

Table 47: Methamphetamine equivalence studies using quantitative values obtained from calibration curves constructed in meconium (MC) and blood (BC) ....................................... 183

Table 48: Exogenous compounds included in selectivity quality control standards. ................. 184

Table 49: Amphetamine selectivity studies using blank meconium from five different sources and blank meconium specimens spiked with various exogenous interferences. ........................ 185

Table 50: Methamphetamine selectivity studies using blank meconium from five different sources and blank meconium specimens spiked with various exogenous interferences. ........... 185

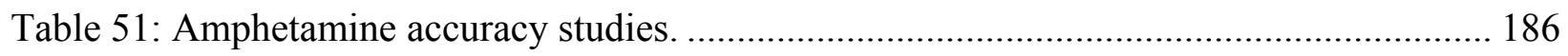

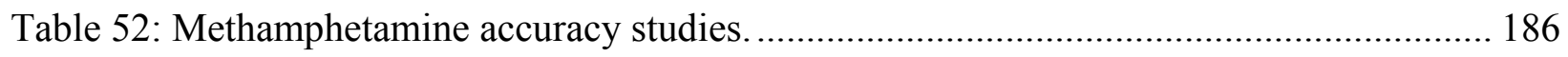

Table 53: Intra- and inter-batch precision studies for amphetamine. ....................................... 186

Table 54: Intra- and inter-batch precision studies for methamphetamine. .................................. 187

Table 55: Optimized MS/MS parameter employed for all DESI experiments........................... 224

Table 56: Analyte concentrations chosen for initial DESI-MS/MS experiments........................ 225

Table 57: Immunoassay screening results for experimental specimens.................................... 251

Table 58: DESI-MS/MS screening results for authentic meconium specimens $(\mathrm{n}=21)$ screened

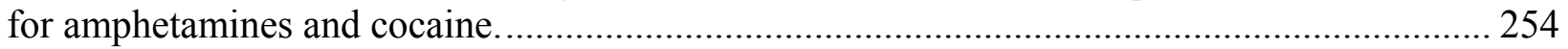


Table 59: Immunoassay (ELISA) screening results for 21 authentic meconium specimens. .... 255

Table 60: DESI-MS/MS screening results for 21 authentic meconium specimens.................. 255 


\section{List of Figures}

Figure 1: Van Deemter plot (Adapted from Waters, 2004) ....................................................... 30

Figure 2: Schematic of an electrospray interface (ESI) (Adapted from Slobodnik 1995)............ 45

Figure 3: Relationship between molecular mass and analyte polarity for analytes suited to GC/MS and different LC/MS interfaces (Adapted from Maurer 1998)..................................... 49

Figure 4: Schematic of electrospray ionization (ESI) showing the three steps of ionization: 1.) Nebulization of a sample solution into electrically charged droplets, 2.) Solvent evaporation, and 3.) Ion ejection from the highly charged droplets (Adapted from Lam 2003) ............................. 50

Figure 5: Schematic of a quadrupole mass analyzer............................................................ 57

Figure 6: Mathieu stability diagram of two masses (A and B) in a quadrupole mass filter. Voltage coordinates corresponding to points under the stability curve represent stable trajectories while all other voltage conditions result in unstable ion trajectory in the quadrupole device................55

Figure 7: Schematic representation of a triple quadrupole mass spectrometer. ……………......... 61

Figure 8: Schematic representation of product (daughter) ion scanning mode. ............................. 63

Figure 9: Schematic representation of precursor ion scanning mode. ............................................ 64

Figure 10: Schematic representation of multiple reaction monitoring (MRM) mode.................... 65

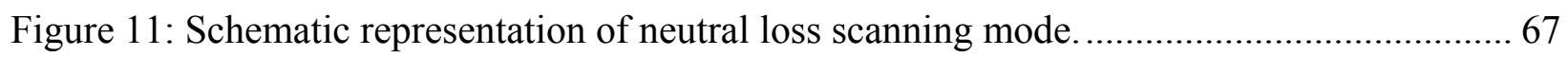

Figure 12: Schematic representation of the Waters TQD ion optics........................................ 72

Figure 13: Number of LC-MS/MS articles appearing in pubmed from 2001 - 2008 for the

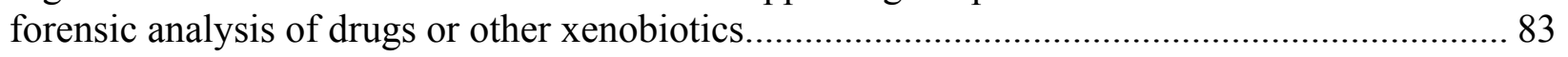

Figure 14: Schematic representation of the 'T-mixing' mode which allows for the simultaneous infusion of analyte solution from reservoir A and mobile phase eluting from the UPLC column.

Figure 15: TIC for the chromatographic separation of eight tricyclic antidepressants on a Waters Acquity TQD. Analytes are not chromatographically resolved but can be quantified using their individual MRM ion traces. 103

Figure 16: Extracted in traces for each analyte. Individual ion traces are acquired in the MRM mode and eliminate the need for chromatographic resolution. 104

Figure 17: TIC from the UPLC separation of cocaine and benzoylecgonine. 125 
Figure 18: Fragmentation pathways for Cocaine and Benzoylecgonine during tandem mass spectrometry experiments[219]........................................................................................ 127

Figure 19: Cocaine recovery at three concentrations over the linear range............................... 132

Figure 20: Benzoylecgonine recovery at three concentrations over the linear range................. 133

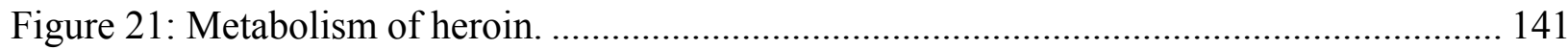

Figure 22: Biotransformation and conjugation of morphine. .................................................. 143

Figure 23: Metabolism of hydrocodone to hydromorphone........................................................ 145

Figure 24: TIC from the UPLC separation of morphine, hydromorphone, codeine, and 6monoacetylmorphine (6-MAM)......................................................................................... 152

Figure 25: Fragmentation pathways for morphine, codeine, hydromorphone, and 6monoacetylmorphine during tandem mass spectrometry experiments[244] ............................. 154

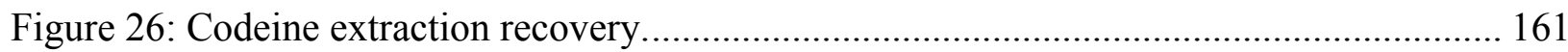

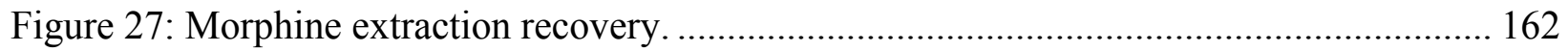

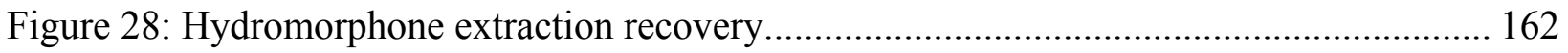

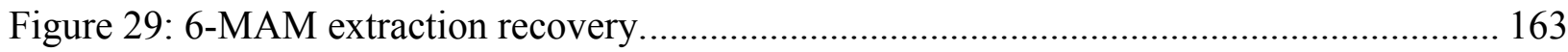

Figure 30: Fragmentation pathways for amphetamine and methamphetamine during tandem mass spectrometry experiments[261]. 175

Figure 31: TIC of UPLC separation \#1. Elution gradient failed to resolve amphetamine (Amp) and methamphetamine (Mamp).

Figure 32: TIC of UPLC separation \#2. Elution gradient resulted in the co-elution of amphetamine (Amp) and methamphetamine (Mamp)......................................................... 178

Figure 33: TIC of UPLC separation \#3. Elution gradient was unable to resolve amphetamine (Amp) and methamphetamine (Mamp). 179

Figure 34: TIC of UPLC separation \#4. Elution gradient was unable to resolve amphetamine (Amp) and methamphetamine (Mamp). 180

Figure 35: TIC of UPLC separation \#5. Elution gradient resulted in significant peak broadening. 181

Figure 36: TIC of UPLC separation \#6. Elution gradient allowed for the baseline resolution of amphetamine (Amp) and methamphetamine (Mamp) 182

Figure 37: Amphetamine recovery. 188 
Figure 38: Methamphetamine recovery. 188

Figure 39: Schematic representation of desorption electrospray ionization (Adapted from Takats 2004). 192

Figure 40: Schematic representation of MALDI ionization region (Adapted from www.magnet.fsu.edu/.../ionization_maldi.html). 194

Figure 41: Proposed procedure for the preparation of meconium extracts for immunoassay analysis (Adapted from ElSohly et al., 1999). 205

Figure 42: Extraction flow chart for a broad based GC-MS screen. 209

Figure 43: Schematic representation of the DESI ion source illustrating geometric parameters $\alpha$ (incident angle), $\beta$ (collection angle), d1 (tip-to-surface distance), and d2 (MS inlet-to-surface distance) (Adapted from Takats et al. 2005)..... 220

Figure 44: Mass spectral responses for all seven analytes using DESI-MS/MS. 224

Figure 45: Analyte signals following direct analysis of spiked meconium samples. 226

Figure 46: Effect of meconium matrix on morphine, hydromorphone, and codeine signal. Mass spectral signals for neat drug standards prepared at $500 \mathrm{ng} / \mathrm{mL}$ can be seen in channels $1-4$ while analyte signals for spiked meconium standards containing $500 \mathrm{ng} / \mathrm{mL}$ of each analyte can be seen in channels 5-8. 228

Figure 47: Effect of meconium matrix on amphetamine and methamphetamine signal. Mass spectral signals for neat drug standards prepared at $200 \mathrm{ng} / \mathrm{mL}$ can be seen in channels 1-4 while analyte signals for spiked meconium standards containing $200 \mathrm{ng} / \mathrm{mL}$ of each analyte can be seen in channels $5-8$. 229

Figure 48: Effect of meconium matrix on cocaine and benzoylecgonine signal. Mass spectral signals for neat drug standards prepared at $200 \mathrm{ng} / \mathrm{mL}$ can be seen in channels $1-4$ while analyte signals for spiked meconium standards containing $200 \mathrm{ng} / \mathrm{mL}$ of each analyte can be seen in channels 5-8. 230

Figure 49: DESI-MS/MS signal for the amphetamines following limited sample preparation according to sample preparation method $\# 1$. 232

Figure 50: DESI-MS/MS signal for cocaine and benzoylecgonine following limited sample preparation. 233

Figure 51: Previously prepared positive quality control (QC) samples were spotted in the first two sample channels to assist with identifying positive authentic samples spotted in channels 3-6. 236 
Figure 52: Sample channels 1-2 represent analyte responses for a cocaine and benzoylecgonine quality control (QC) standard (50 ng/mL) while sample channels 3-6 were spotted with spiked meconium samples $(250 \mathrm{ng} / \mathrm{mL})$. 237

Figure 53: Mass spectral responses for a cocaine/benzoylecgonine positive control $(50 \mathrm{ng} / \mathrm{mL})$ in channels 1-3 and analyte responses for a blank meconium specimen spotted in channels 4-6. . 239

Figure 54: Mass spectral response of an amphetamine/methamphetamine positive control sample (50 ng/mL) spotted in channels 1-2 and analyte responses for a meconium specimen spiked with amphetamine and methamphetamine $(500 \mathrm{ng} / \mathrm{mL})$ in channels 3-6. 240

Figure 55: Mass spectral response of an amphetamine/methamphetamine positive control sample $(50 \mathrm{ng} / \mathrm{mL})$ spotted in channels $1-2$ and analyte responses for a blank meconium specimen spotted in channels 3-6.

Figure 56: Mass spectral response of an opiate positive control sample $(500 \mathrm{ng} / \mathrm{mL})$ spotted in channels 1-2 and analyte responses for a meconium specimen spiked with opiates $(500 \mathrm{ng} / \mathrm{mL})$ and spotted in channels 3-6.

Figure 57: Mass spectral responses of amphetamine and methamphetamine standards (a-b) as well as benzoylecgonine and cocaine standards (c-d) spotted in channel 2. Mass spectral responses in channels 4-6 represent meconium samples which had been spiked with amphetamine and methamphetamine $(500 \mathrm{ng} / \mathrm{mL})$ and prepared according to sample preparation method \#1. Mass spectral responses in channels 7-9 represent meconium specimens which had been spiked with amphetamine and methamphetamine $(500 \mathrm{ng} / \mathrm{mL})$ and prepared according to sample preparation method $\# 2$. 244

Figure 58: Mass spectral responses for amphetamine and methamphetamine with the amphetamine/methamphetamine specific MS/MS method containing 2 MRM transitions. Sample channel 2 contains a positive quality control specimen prepared at $25 \mathrm{ng} / \mathrm{mL}$. Sample channels $4-$ 6 contain meconium matrix spiked with amphetamine and methamphetamine $(500 \mathrm{ng} / \mathrm{mL})$ and prepared according to sample preparation method \#1. Sample channels 7-9 contain meconium matrix spiked with amphetamine and methamphetamine $(500 \mathrm{ng} / \mathrm{mL})$ and prepared according to sample preparation method \#2. 246

Figure 59: Mass spectral responses of amphetamine and methamphetamine standards (a-b) as well as benzoylecgonine and cocaine standards (c-d) spotted in channel 2. Mass spectral responses in channels 4-6 represent meconium samples which had been spiked with cocaine and benzoylecgonine $(250 \mathrm{ng} / \mathrm{mL})$ and prepared according to sample preparation method \#1. Mass spectral responses in channels 7-9 represent meconium specimens which had been spiked with cocaine and benzoylecgonine $(250 \mathrm{ng} / \mathrm{mL})$ and prepared according to sample preparation method \#2. 247

Figure 60: Mass spectral responses of benzoylecgonine and cocaine standards (a-b) spotted in channel 2. Mass spectral responses in channels 4-6 represent unfiltered meconium samples which had been spiked with cocaine and benzoylecgonine and analyzed using the cocaine/BE specific MS/MS method consisting of one MRM transition for each analyte. Mass spectral 
responses in sample channels 7-9 represent filtered meconium specimens analyzed by the same method...... 248

Figure 61: Positive quality control specimens were spotted in channel 2 and analyzed first to aid with positive identifications. Authentic meconium specimens were then spotted in every second sample channel to reduce the possibility of sample redistribution.

Figure 62: Show the mass spectral responses for amphetamine (a) and methamphetamine (b) in the positive quality control and four negative specimens. Signal intensities for the positive controls were not dissimilar to those of negative specimens. 257

Figure 63: Shows the mass spectral responses for benzoylecgonine (a) and cocaine (b) in a positive quality control specimen, three negative specimens $(1,2$, and 3$)$ and a true positive specimen (4). Intense analyte signals in all true positives allowed for easy identification. ....... 258 


\section{List of Abbreviations, Symbols/Nomenclature}

$\mu$ - Mean

$\mu$ - Mobile flow velocity

$\mu \mathrm{g}$ - Microgram

$\mu \mathrm{L}$ - Microliter

6-MAM - 6-monoacetylmorphine

AAFS - American Academy of Forensic

Sciences

APcI - Atmospheric pressure chemical ionization

API - Atmospheric pressure ionization

BC - Blood calibration curve

$\mathrm{BEH}$ - Bridged ethyl hybrid

CAN - Acetonitrile

CI - Chemical ionization

CID - Collision induced dissociation

CNS - Central nervous system

CV - Coefficient of variation

d1 - Tip-to-surface distance

d2 - MS inlet-to-surface distance

DATs - Dopamine reuptake transporters

DAWN - Drug Abuse Warning Network

DC - Direct current

DESI - Desorption electrospray ionization

DESI-MS/MS - Desorption electrospray

ionization-Tandem mass spectrometry

DFSA - Drug facilitated sexual assault

dp - Particle size

ED - Emergency department

EDDP - 2-ethylidene-1,5-dimethyl-3,3-

diphenylpyrrolidine

EI - Electron ionization

ELISA - Enzyme-linked immunosorbent

assay

EME - Ecgonine methyl ester

EMIT - Enzyme-multiplied immunoassay technique

EPI - Enhanced product ion scan

ESCI - Electrospray chemical ionization

ESI - Electrospray ionization

FAB - Fast atom bombardment ionization

FDA - Food and Drug Administration
FIA - Flow injection analysis

Fopt - Optimum flow rate

FPIA - Fluorescent polarization

immunoassay

g- Gram

GC - Gas Chromatography

GC/MS - Gas Chromatography-Mass

Spectrometry

GCP - Glycerol phosphatidylcholine

phospholipids

$\mathrm{H}$ - Height equivalent to an effective theoretical plate

$\mathrm{H}_{2} \mathrm{O}$ - Water

HETP - Height equivalent to a theoretical

plate

HPLC - High Performance Liquid

Chromatography

HQC - High quality control

ICR - Ion cyclotron resonance

IDA - Information-dependant acquisition

$\mathrm{kV}-$ Kilovolts

L - Column length

LACCMEC - Los Angeles County Office of

Chief Medical Examiner-Coroner

LC - Liquid Chromatography

LC/MS - Liquid Chromatography-Mass

Spectrometry

LC/MS/MS - Liquid Chromatography-

Tandem Mass Spectrometry

LLE - Liquid-liquid extraction

LLOQ - Lower limit of quantitation

LOD - Limit of detection

LQC - Low quality control

LSD - Lysergic acid diethylamide

$\mathrm{m} / \mathrm{z}$ - Mass to Charge Ratio

MALDI - Matrix assisted laser desorption

MAO - Monoamine oxidase

$\mathrm{MC}$ - Meconium calibration curve

MCSHC - Maternal and children's special health care

MDA - Methylenedioxyamphetamine 
MDMA -

Methylenedioxymethamphetamine

$\mathrm{MeOH}$ - Methanol

mg - Milligram

$\mathrm{mL}$ - Milliliter

MRM - Multiple reaction monitoring

MS - Mass Spectrometry

MS/MS - Tandem Mass Spectrometry

$\mathrm{N}$ - Number of theoretical plates

$\mathrm{NaOH}$ - Sodium hydroxide

ng - Nanogram

NIDA - National Institute on Drug Abuse

NSDUH - National survey on drug use and health

OTC - Over the counter

PB - Particle beam ionization

PDA - Phase doppler anemometry

PDA - Photodiode array

QC - Quality control

QTOF - Quadrupole-Time of flight mass

spectrometer

RE - Relative error

RF - Radiofrequency

RIA - Radioimmunoassay

Rs - Resolution

RSD - Relative standard deviation

$\mathrm{S} / \mathrm{N}$ - Signal to noise ratio

SD - Standard deviation

SIDS - Sudden infant death syndrome

SIM - Single ion monitoring

SIMS - Secondary ions mass spectrometry

SLE - Supported-liquid extraction

SNaRIs - Serotonin noradrenergic reuptake inhibitors

SOFT - Society of Forensic Toxicologists

SPE - Solid phase extraction

SRIG - Stacked ring ion guide

SRM - Selected reaction monitoring

SSRIs - Selective serotonin reuptake

inhibitors

TIC - Total ion chromatogram

TMF - 3-methylfentanyl
TOF - Time-of-flight

TP - Thermospray ionization

TQD - Triple quadrupole detector

tR - Retention time

T-Wave ${ }^{\mathrm{TM}}$ - Travelling Wave ${ }^{\mathrm{TM}}$

TWIG - Travelling Wave ${ }^{\mathrm{TM}}$ ion guide

UCT - United Chemical Technologies

ULOQ - Upper limit of quantitation

UPLC- Ultra Performance Liquid

Chromatography

UPLC-MS/MS - Ultra Performance Liquid

Chromatography-Tandem Mass

Spectrometry

UV - Ultra violet

$\mathrm{V}-$ Volts

$\alpha$ - Incident angle

$\beta$ - Collection angle

$\sigma$ - Standard deviation 


\section{Chapter 1: Introduction to Prenatal Substance Abuse and Its Clinical and Forensic Significance}

\subsection{Introduction}

Prenatal substance abuse is an ongoing concern due to the characteristic physical and mental developmental problems that result from drug abuse during pregnancy. Illicit drug consumption continues to increase across geographical, social, and cultural groups worldwide and in 2000 The World Drug Report estimated 180 million people, or 3\% of the worlds inhabitants to be drug users[1]. Substance abuse in the United States has reached epidemic proportions during the past two decades and in 2006 an estimated 20.4 million Americans aged 12 years or older were users of illicit drugs[2, 3]. This estimation represents $8.3 \%$ of the American population aged 12 years or older[2]. Females constitute approximately $30 \%$ of the substance addicted population in the United States and most are of childbearing age[4]. Among pregnant women aged 15 to 44 years who participated in the 2006 National Survey on Drug Use and Health (NSDUH), 4.0\% reported having used illicit drugs within 1 month of the survey[2]. National findings from the 2006 survey indicate that the rate of illicit drug use among pregnant women aged 15 to 44 years has remained steady since 2003[2].

Estimating the prevalence of drug use among pregnant women based on maternal history or broad scale surveys often proves unreliable due to guilt, embarrassment, fear of reprisal, or of loss of custody[5]. As a result, identification of the drug exposed neonate is a difficult task and even in the case of maternal admission, information regarding the type and/or extent of drug use is often inaccurate[6]. One survey based on maternal report estimated that the prevalence of drug abuse among pregnant women ranged between $0.4 \%$ and $27 \%[6,7]$. A survey conducted on 
infants delivered at a tertiary perinatal center illustrated the inaccuracy of estimations based on maternal history. While only $10.5 \%$ of infants were birthed to mothers suspected of drug use based on maternal history, subsequent drug testing revealed that $42 \%$ of infants had been exposed to illicit drugs[8]. Another study of 3000 infants illustrated the inaccuracies associated with maternal self-report. Of the 3000 infants monitored in the study, $43 \%$ were found to be positive for illicit substances through toxicological analysis, while only $11 \%$ of these were reported by the mother[9].

A study conducted on the prevalence of cocaine use during pregnancy in the early 1990s reported that 37 out of $600(6.25 \%)$ infants born across three metropolitan hospital nurseries in the Toronto area tested positive for cocaine[10]. These findings were consistent with estimations made by Birchfield et al who concluded that rates of infants exposed prenatally to cocaine range between $2.6 \%$ and $11 \%$ of all live births[11]. A nationwide survey carried out in the early $1990 \mathrm{~s}$ at urban teaching hospitals indicated that $10-45 \%$ of the women cared for at those hospitals use cocaine during pregnancy[12]. A separate survey of 36 hospitals reported that $11 \%$ of the women studied had used illicit drugs during pregnancy[7]. A follow up report by the same authors in 1992 estimated that between 500,000 and 750,000 newborns are exposed to illicit drugs each year[13].

Various neonatal health and developmental problems are thought to be directly related to fetal exposure to drugs, alcohol, chemical agents, or other xenobiotics [6, 14-16]. Despite extensive research and continued evidence of fetal and neonatal health risks, a large number of pregnant women are involved in illicit drug use[5]. Drug use during gestation is associated with higher risks for poor obstetrical outcomes, including placental abruption, premature labor, low 
birth weight, microcephaly, congenital anomalies, necrotizing enterocolitis, neonatal withdrawal, neurobehavioral effects, subarachnoid and intracerebral hemorrhage, and fetal death[17-25].

Due to the detrimental effects resulting from prenatal exposure to illicit substances, correct diagnosis of drug use during pregnancy is essential. Correct diagnosis and early intervention will allow the child to receive the specialized treatment and the care required to ensure that their development is not further compromised. Successful diagnosis of drug abuse during pregnancy will also assist in preventing the same mother from giving birth to subsequent drug-exposed children[26]. It is extremely important to ensure that the early diagnosis of drug abuse during pregnancy not only brings about beneficial changes in the environment of the infant, but also aids in the successful rehabilitation of substance addicted mothers[27]. Although the early identification of prenatal substance abuse will aid in the long term wellbeing of the infant, without the implementation of a well defined intervention road map, such diagnosis also have the potential to cause harm to mothers, children, and families alike[28]. Careful consideration of the circumstances surrounding each and every positive result will ensure that a mother who has used drugs or alcohol at some time during pregnancy is not mislabeled as a substance abuser in the absence of true abuse. Review of positive results with mothers suspected of substance abuse will help to ensure that infants are not separated from their mothers and placed in living situations that offer no benefit[28].

Traditional identification of neonatal drug exposure is accomplished using a combination of maternal history, newborn clinical symptoms and laboratory toxicology testing of the mother and the infant[27]. Generally, toxicological testing of the mother and infant will only occur if the consulting physician has reason to believe that prenatal substance abuse may be an issue based 
on maternal history, or if the infant's physical features meet certain criteria which are commonly associated with prenatal exposure to drugs.

Many states mandate toxicology testing of all mothers and infants regardless of maternal history or certain physical features of the infant. Indiana's Maternal \& Children's Special Health Care (MCSHC) department mandates laboratory testing of meconium specimens to detect the presence of controlled substances for those infants born in Indiana who meet selected criteria at

birth. Toxicology testing is performed on high-risk newborns (1) whose weight is less than 2500 grams and whose head circumference is smaller than the 10th percentile for the infant's gestational age when there is no other medical explanation for these conditions; or (2) when there is maternal history of current or past drug use; or; (3) mother had no or inconsistent prenatal care (frequently missed appointments, hospital hopping); or (4) infant shows signs/symptoms suggestive of drug effects or withdrawal; or (5) unexpected abruption placentae[29]. Approximately 1,600 newborn infants meet one or more of these criteria in Indiana alone and many additional meconium specimens are submitted for analysis based on recommendations from the consulting physician[29].

\subsection{Drug Transfer in the Maternal-Fetal Complex}

The placenta is a specialized organ which not only plays a lead role in fetal protection during intrauterine life but also continuously adapts itself to meet developmental and nutritional requirements of the fetus while in the uterus[30]. The role of the placenta as a transport organ is crucial for fetal development as it is responsible for transferring oxygen and nutrients to the fetus while simultaneously allowing for the efficient removal of fetal metabolism products by the mother[30, 31]. Essential nutrients such as amino acids, vitamins, phosphate, iron, mono- and 
dicarboxylates, and glucose among others are transferred to the fetus throughout intrauterine life via very specific transport mechanisms present within in the placenta[31].

The developing fetus is not only provided with essential nutrients and oxygen via specific placental transport mechanisms but is also effectively protected through the placentas ability to prevent the entry of various xenobiotics from the maternal blood supply[31]. Excluding drugs of large molecular weight, such as heparin or insulin, most drugs are thought to cross the placental membrane during pregnancy and are associated with varying degrees of fetal exposure[32]. In general, un-ionized molecules with low molecular weights and high lipid solubility will diffuse across the placental membrane more readily than large hydrophilic drug molecules[33]. Drugs that are highly bound to proteins or contain ionized functional groups in plasma $(\mathrm{pH} 7.4)$ will exhibit a much lower degree of placental diffusion than unionized, unbound drugs.

The placenta also facilitates the passage of various xenobiotics form the fetus to the mother for elimination should they initially permeate the placental membrane. It is well documented that various licit and illicit drugs can indeed cross the placental membrane which represents the primary physiological link between mother and fetus [34-38].

While most xenobiotics cross the placental membrane and enter the fetal bloodstream via passive diffusion, other potential transport mechanisms include facilitated diffusion, active transport, and filtration. The mechanism by which certain drugs permeate the placental membrane and enter the fetal bloodstream is dependent on the physicochemical properties of the compounds such as molecular size, polarity, and $\mathrm{pKa}$, as well as lipid solubility and protein binding ability[32]. Maternal pharmacokinetics including the volume of distribution, rate of metabolism and excretion, and the effect of haemodynamic changes in the mother during pregnancy all impact the degree and severity of in utero drug exposure[32]. Maternal blood $\mathrm{pH}$, 
which dictates the state of ionizable functional groups, will also play a significant role in the drugs ability to cross the placenta[34].

Numerous transport systems which recognize a wide variety of pharmacologically active drugs as substrates have been identified in the placenta, along with several additional transport systems whose role in the placenta remains an enigma[31]. There is strong evidence to suggest

that several placental transport systems are direct targets for common drugs of abuse including cocaine, amphetamines, nicotine, and cannabinoids[31]. Interference of drugs of abuse with the transport function of the placenta can occur following a direct interaction of the drug with specific transporters, or through an indirect modification of cell signaling pathways or transmembrane ion gradients which subsequently influences transporter function[31].

Accompanying the recognition that drugs of abuse significantly affect placental transport is the realization that maternal use of such drugs compromises the normal physiological function of the placenta. Unnatural changes in placental physiology resulting from maternal drug use produces deleterious effects in the mother as well as in the developing fetus[31].

\subsection{Traditional Testing Procedures}

Toxicological analysis of maternal and neonatal specimens is an objective means of identifying prenatal drug exposure. Analysis of biological specimens obtained from the neonate is necessary to document proof of the infant's exposure to illicit drugs, even if the mother admits to the use of illicit substances[39]. Positive toxicology testing in the infant facilitates court action in the cases where authorities have recommended the child be removed from the mothers care and placed in foster care[39].

Traditional toxicological analyses aimed at identifying prenatal exposure to illicit substances have utilized various maternal and neonatal specimens over the years. Maternal blood 
is routinely analyzed for the presence of illicit substances in the mother. However, due to the relatively short retention time of most drugs in blood, and the invasiveness of sample collection, blood analysis is somewhat limited. Toxicological analysis of neonatal blood is rarely performed as serum drug levels in the infant largely depend on the time interval between the mother's last drug intake and the subsequent collection of the neonatal blood. This time interval can be substantial and as a result drug levels in the neonatal blood will likely fall below detection limits for common screening techniques[39].

Neonatal urine is the most widely analyzed biological fluid for the determination of in utero drug exposure and offers several distinct advantages over blood. Drug concentrations in neonatal urine will be higher than those in corresponding blood (serum) specimens due to the concentrating ability of the kidneys[40]. Neonatal urine also offers a slightly longer window of detection than blood and should indicate the presence of any substances that the neonate was exposed to in the last 3-7 days prior to delivery[41]. Urine also offers a larger volume for collection than blood and from a toxicological standpoint it is easier to analyze than blood because it is devoid of protein and cellular constituents that can complicate extraction and/or analysis techniques[40].

Although neonate urine has been the most widely analyzed biological fluid for the identification of in utero exposure to drugs, it does have several disadvantages which can complicate collection and subsequent analyses. Neonatal urine collection is difficult, invasive and must be performed as close to birth as possible as appreciable levels of drugs and/or metabolites are expected to be present in the first specimen only[39, 41]. Drugs present in the infant's urine represent recent drug use by the mother and depending on the physicochemical properties of the drug, the urine may test negative if the mother is an infrequent user or abstained 
from use in the days leading up to delivery[39, 41]. Another common drawback to neonatal urine analysis is the fact that most laboratories adopt pre-existing urine-based methodologies to screen the infant's urine for drugs of abuse. Unfortunately, neonatal urine is far from an ideal specimen for such analyses. Pre-existing urine-based methodologies have been widely developed and validated for use in workplace or forensic drug analysis[42]. Such screening techniques adopt cutoff levels deemed suitable for their intended purpose and will most likely be too high for the drug concentrations in clinical samples such as neonatal urine. Drug concentrations in neonatal urine following in utero exposure would be expected to be lower than concentrations seen in positive workplace or forensic samples due to maternal-fetal transfer kinetics, maternal abstinence or infrequent use, and compromised sample collection at the time of birth. As a result of these factors, several workplace drug testing thresholds have been shown to be too high for clinical samples. The consequence of such false negatives is an underestimation of drug exposure in the clinical setting. Multiple studies indicated that adopting lower cutoff levels for such assays can dramatically improve detection rates[43, 44]. Due to the relative short detection window offered by neonatal urine and a lack of recommended cutoff levels which take into account the pharmacokinetics of drug transfer and elimination, the incidence of false-negative results in neonatal urine analysis is high and can range from $32 \%-63 \%[41,45]$.

Recently, meconium has become the specimen of choice for the detection of prenatal exposure to several drugs of abuse[26, 46]. Meconium is the first blackish tarlike material passed from the rectum by the newborn and is not fully evacuated until 125 hours post natal [47-49]. Meconium is a dark-green mass of water, epithelial cells, mucopolysaccharides, bile pigments, and other lipids which begins to form between the $12^{\text {th }}$ and $16^{\text {th }}$ week of gestation and accumulate until birth. Formation of meconium occurs in the fetal gut and results from 
swallowed amniotic fluid and sloughed gastrointestinal epithelial cells[50]. Accumulation of drugs and other xenobiotics occurs in the meconium as a result of fetal swallowing. This phenomenon occurs when the fetus releases urine, containing drugs and metabolites, into the amniotic fluid where it is subsequently swallowed and deposited into the meconium. Subsequent exposure, excretion and reabsorption through fetal swallowing, combined with maternal metabolism and elimination, and placental transfer results in the concentration of drugs and their metabolites in meconium[51,52]. At approximately the $16^{\text {th }}$ week of gestation, the fetus possesses fully functional liver enzymes capable of metabolizing drugs, allowing for excretion into the bile and urine. Meconium is one of the most sensitive matrices for the detection of prenatal drug exposure due to the accumulation of substances over several months of gestation.

The usefulness of meconium as an alternative toxicological specimen was first demonstrated by Ostrea et al[53] in the late 1980's, and its popularity as a tool for the identification of prenatal exposure has continued to increase over the past two decades for several reasons. The first reason is the relatively simple and non-invasive procedure used to collect meconium samples, making it more successful than urine collection[54]. Meconium analysis also extends the window of drug detection to approximately the last 20 weeks of gestation as well as extending the window for specimen collection.

Meconium analysis has become an extremely important tool for the identification of prenatal exposure to drugs of abuse. While the analysis of maternal and newborn blood or urine can be useful for identifying recent (1-4 days) drug use, mothers who have abstained from use in previous days will likely go undetected using these traditional matrices. However, due to nature of meconium formation, which occurs over several months of gestation, isolated drug use during the second and third trimesters will still be detectable through meconium analysis long after 
drugs have been metabolized and excreted from the blood and urine. While blood and/or urine analysis proves useful for the identification of recent or chronic drug use, the extended detection window afforded by meconium analysis aids in identifying infants exposed to drugs through more isolated or recreational drug use which has previously gone undetected.

\subsection{History of Meconium Analysis}

The biochemical analysis of meconium for medical, clinical, and forensic purposes has been widely reported in the scientific literature for over a century. While this review will focus on the analysis of meconium for the purpose of identifying prenatal exposure to xenobiotics, various scientific reports have utilized meconium analysis for alternative purposes. Meconium analysis has been reported for the purpose of determining its metallic content[55], elucidating its chemical composition in relation to blood-group-specific polysaccharides and abnormalities in cases of meconium ileus[56], determining its tryptic activity in cases of congenital intestinal obstruction[57], and isolating and preparing blood group substances for the purposes of immunization studies[58].

One of the first reported uses of meconium as an alternate toxicological specimen was published in 1956 by Kinsella and coworkers who wished to study the enteric excretion of metabolites of steroid hormones in the human[59]. Although the estrogenic activity of meconium had been previously reported by Gsell-Busse and coworkers[60], this was the first report of the successful isolation and detection of estrogens in meconium. The authors theorized that the isolation and detection of estriol should be possible due to the fact that meconium constitutes a concentrate and a record of enteric excretion of metabolites throughout the fetal life. Results indicated that estriol is present in the human intestinal content both as a free compound and as a glucuronide conjugate, and its isolation and detection from meconium is feasible. The authors 
conclude that meconium should prove to be a fruitful biological material not only for the further investigation of steroid hormone metabolism but for other compounds of pharmacological interest[59].

Four years later, the same authors applied a similar methodology for the isolation and determination of dehydroepiandrosterone from meconium[61]. While the determination of dehydroepiandrosterone supported the authors earlier theory that meconium constitutes a record of enteric excretion over the fetal lifetime, the absence of androsterone, a subsequent metabolite of dehydroepiandrosterone, indicated that vast differences may exist between fetal and adult intermediary metabolism.

The potential value of meconium, not only as an alternate biological material for metabolic investigations, but also as a diagnostic tool to better understand the endocrinology of the fetus was being realized by the early 1960s thanks largely in part to the work of Kinsella et al. Following this work, several reports appeared in the scientific literature describing analytical methodologies for the isolation and determination of steroid hormones and their conjugates in human meconium for the purpose of endocrinology research [62-65]. Green and Shwachman proposed the use of presumptive testing for the purpose of identifying cystic fibrosis on the basis of high serum protein in meconium specimens[66]. The authors highlight the ongoing use of protein analysis for the identification of meconium ileus and suggest that abnormal serum protein levels in meconium may be a useful biomarker of cystic fibrosis in patients who did not have the complication of meconium ileus[66].

Meconium was also utilized for the successful determination of listeria monocytogenes for the purpose of identifying listeriosis in the newborn infant[67]. In 1968, Miettinen and coworkers published one of the first studies on the application of gas chromatography and mass 
spectrometry for the detection of sterols in several fetal specimens including vernix caseosa, amniotic fluid, and meconium[68].

The first reported use of meconium as an alternative toxicological specimen for the detection of drug of abuse was published in 1987 by Ostrea et al who successfully detected heroin, cocaine, and cannabinoid metabolites in the stools of infants born to drug dependant mothers[69]. In the following year, Ostrea and coworkers described a method for the rapid isolation and detection of morphine and benzoylecgonine in meconium using a simple liquid extraction followed by radioimmunoassay screening and suggested that such a technique would prove superior for the detection of drug metabolites in meconium due to the low incidence of false positive results which tend to be common in urine analysis[41, 53].

Ostrea continued to publish methodologies for the detection of drugs of abuse in meconium as alternatives to the more traditional urine analysis and in 1989 he and coworkers analyzed 20 meconium specimens obtained from drug dependant mothers to compare the analytical results with those obtained by urine analysis[45]. Of the 20 meconium specimens analyzed in the study, all were positive for at least one drug metabolite using radioimmunoassay (RIA) techniques. The widespread popularity of cocaine in the late 1980 s was evident with $80 \%$ of the specimens screening positive for benzoylecgonine while $55 \%$ of the specimens were positive for morphine and $60 \%$ were indicative of cannabinoid use. Meconium specimens were analyzed over the first three days of evacuation and while drug concentrations were highest during the first two days of collection, positive determinations were still reported on the third day of collection. Analysis of the urine only yielded positive results for $37 \%$ of the infants using fluorescent polarization immunoassay (FPIA) and when paired urine and meconium specimens were analyzed using RIA, higher concentrations of drug metabolites were detected in meconium. 
Interestingly, eight of the urine samples screened negative despite having a positive meconium result indicating that meconium constitutes a very useful matrix for drug screening in the neonate[45].

Maynard and Amoruso also investigated the usefulness of meconium as a matrix for identifying prenatal exposure to drugs in abuse in 28 specimens collected from neonates born to women suspected of drug abuse[70]. Urine collected from the newborn, the mother, or both, along with meconium specimens were analyzed for the presence of cocaine, morphine, codeine and marijuana. Results obtained through the analysis of meconium specimens were concordant with urine analysis in $86 \%$ of the cases and in three cases meconium analysis yielded positive results for cocaine while the newborn urine specimens were negative. The authors conclude that due to the ease and reliable nature of meconium collection and the compatibility of this type of screen testing with high throughput commercial laboratories, meconium is a useful specimen for the detection of drugs in newborns[70].

In 1991, Ostrea and Welch reviewed current analytical methodologies for the identification of maternal drug abuse and highlighted the importance of utilizing alternate specimens such as meconium or hair as recent literature suggested that urine analysis may seriously under diagnose the prevalence of fetal exposure to various drugs[71]. In the following year, Ostrea and coworkers published the first large-scale, prospective, epidemiological study into the prevalence and characteristics of maternal drug use in a high-risk, urban population by analyzing the meconium from more than 3000 neonates delivered in a single perinatal center over a ten month period[8]. Meconium specimens were analyzed were cocaine, morphine and cannabinoids using RIA. Of the 3010 subjects, $44 \%$ were positive for one or more of the drug classes while only 335 (11\%) of the mothers actually admitted to maternal drug use. Of the 335 
mothers who admitted drug use, $52 \%$ of their newborns returned positive urine samples while $88 \%$ of the infants tested positive through meconium analysis. Due to the often normal appearance of infants who have been prenatally exposed to drugs and whose mothers deny such use, there is a growing need to more effectively identify the drug exposed child. The authors conclude that improved identification of exposed newborns is possible with meconium drug analysis[8].

Callahan and coworkers evaluated the sensitivity of meconium analysis compared directly to hair and urine analysis for the detection of gestational exposure to cocaine[47]. The meconium, hair, and urine of 59 infants who had been prenatally exposed to cocaine were collected and analyzed using GC/MS, RIA and immunoassay, respectively. The authors determined the most sensitive matrices for the identification of prenatal drug exposure to be meconium or hair as traditional immunoassay analysis of the infants urine failed to identify $60 \%$ of the cocaine-exposed infants. GC/MS analysis of the meconium or RIA analysis of the infants hair appears to be capable of providing sufficient sensitivity required to identify fetal cocaine exposure which occurred during the last two trimesters of pregnancy[47].

In 1992, Dahlem and colleagues published the results of yet another comparative study aimed at assessing the suitability of meconium relative to infant urine for the identification of in utero drug exposure[72]. The suitability of meconium relative to urine was assessed to identify the most accurate way of determining maternal drug use as maternal admissions often prove unreliable. Meconium and urine specimens were collected from twenty infants born to drug dependant mothers and analyzed using RIA. Nineteen of the twenty infants (95\%) tested positive for drugs in meconium while only thirteen (65\%) tested positive through immunoassay analysis of the urine. Urine analysis failed to detect the presence of morphine in three specimens, cocaine 
in two specimens, and cannabinoids in three specimens. Results indicated that meconium is not only easier and less invasive to collect, but it also constitutes a reliable alternate matrix to urine for the detection of drugs in the neonate[72].

Yawn and coworkers employed meconium screening in combination with urine analysis for the purpose of assessing substance use in rural Midwestern pregnant women[73]. Meconium specimens screened positive for cannabinoids in $1.1 \%$ of subjects, opiates in $0.6 \%$ of subjects, and cocaine in $0.1 \%$ of samples indicating that while substance use was relatively low in this particular rural area, meconium analysis provides a reliable technique for the detection of such use[73].

By the mid 1990s, meconium was widely recognized as a sensitive matrix for the determination of prenatal drug exposure. The toxicological analysis of meconium specimens became more common in studies aimed at determining rates of maternal substance use rather than simply assessing the suitability of meconium for such analysis. In 1993, Dusick and coworkers employed meconium analysis to determine the association between prenatal cocaine exposure and intracranial ultrasonographic abnormalities, among other perinatal outcomes[74]. HPLC and GC/MS analysis of meconium specimens collected from 323 consecutively born very low birth weight infants enabled the researchers to assign infants to either a cocaine-exposed group, or a cocaine-nonexposed group to further study the relationship between prenatal substance abuse and adverse perinatal outcomes. Studies indicated that while cocaine does not appear to increase the incidence or severity of intracranial hemorrhage or periventricular leukomalacia, it does increase the risk of abruptio placentae, surgical ligation of a patent ductus arteriosus and seizures in very low birth weight infants. Meconium analysis will prove instrumental in identifying infants predisposed to such outcomes[74]. 
Rosengren and colleagues utilized meconium analysis to determine the prevalence of cocaine use and assess the perceptions and pitfalls associated with current predictors of maternal use[75]. Results indicated that meconium analysis is necessary to positively identify cocaine exposed infants as the more traditional predictors, such as the opinion of the nurse, can be frequently inaccurate. Lombarderoa and colleagues reported a sensitive GC/MS methodology for the detection of cocaine, benzoylecgonine and ecgonine methyl ester (EME) in meconium extracts taken directly from diapers of exposed infants[76]. The authors report a LOD of 11 $\mathrm{ng} / \mathrm{mL}$ and conclude that $\mathrm{GC} / \mathrm{MS}$ analysis of meconium provides an attractive alternative to urine for the detection of cocaine and its metabolites as meconium collection is less invasive and less discomforting for the neonate[76].

In 1994, following the publication of several methodologies for the detection of drugs in meconium specimens, Wingert and colleagues conducted a large scale study aimed at comparing meconium, maternal urine, and neonatal urine as matrices for the detection of maternal drug use during pregnancy[77]. Meconium, maternal urine and neonatal urine were collected from 423 consecutive deliveries at a large, metropolitan obstetric hospital. Specimens were screened for cocaine, cannabinoids, codeine, morphine, and methadone using EMIT and positive screens were confirmed using GC/MS. Of the three matrices under investigation, meconium proved more reliable than maternal or neonatal urine for the detection of benzoylecgonine and was equally effective for the determination of codeine, morphine, cannabinoids and methadone[77]. Similar publications highlighting the usefulness of meconium for the sensitive detection of drugs of abuse lead to the widespread application of meconium analysis in both academic and health institutions worldwide [44, 46, 78-88]. 
Many early methodologies employed GC/MS for the determination of analytes in meconium. This was largely due to the complex nature of the meconium matrix which meant that chromatographic separation was required to isolate the analytes and mass spectral information was required for unequivocal identification. Chromatographic techniques coupled to mass spectrometric detectors have continued to find widespread use in forensic toxicology laboratories for the detection of drugs of abuse in meconium.

\subsubsection{Instrumental Methodologies Employed for the Analysis of Meconium}

A large majority of analytical methodologies reported in the literature describing the detection of xenobiotics in meconium have utilized GC/MS. Recent advances in analytical technologies have allowed for the application of even more sensitive and selective assays to the problem of identifying prenatal drug exposure in the neonate. In 2003, Pichini et al described a sensitive methodology for the detection of arecoline in meconium using high performance liquid chromatography (HPLC) coupled to a quadrupole mass spectrometer which was equipped with an electrospray ionization (ESI) source[89]. Chromatographic separation was achieved using a reversed phase column and the mass spectrometer was operated in single ion monitoring (SIM) mode allowing for a lower limit of quantitation (LLOQ) of $5 \mathrm{ng} / \mathrm{g}$.

One of the earliest applications of tandem mass spectrometry for the detection of xenobiotics was published in 2005 by Choo and coworkers who employed LC-APCI-MS/MS for the quantification of methadone and its metabolites following solid phase extraction (SPE) from human meconium[90]. The authors report a LLOQ of $5 \mathrm{ng} / \mathrm{g}$, highlighting the enhanced sensitivity of the tandem mass spectrometer. 
In 2006, Kato and colleagues reported a HPLC-MS/MS methodology employing isotope dilution and an on-line SPE for the sensitive determination of phthalate metabolites in meconium[91]. The efficiency of the solid phase extraction combined with the inherent sensitivity of tandem mass spectrometry enabled the authors to achieve LLOQs of between 0.2 and $0.7 \mathrm{ng} / \mathrm{g}$. Since the appearance of these early studies in the scientific literature, tandem mass spectrometry has allowed for the low level detection of various analytes of forensic interest such as codeine, morphine, hydrocodone, hydromorphone, oxycodone, 6-acetylmorphine[92], amphetamine, 4hydroxy-3-methoxyamphetamine, 4-hydroxy-3-methoxymethamphetamine, methamphetamine, 3,4-methylenedioxyamphetamine, 3,4-methylenedioxyethylamphetamine, 3,4methylenedioxymethamphetamine, norephedrine, $p$-hydroxyamphetamine, $p$ hydroxymethamphetamine[93], alpha-hydroxyalprazolam, alpha-hydroxyethylflurazepam, alphahydroxytriazolam, alprazolam, desalkylflurazepam, diazepam, lorazepam, midazolam, nordiazepam, oxazepam, temazepam, clonazepam, 7-aminoclonazepam[94], nicotine, cotinine, trans-3'-hydroxycotinine, nornicotine, norcotinine[95], buprenorphine, and norbuprenorphine[96].

While meconium analysis is today performed largely for the purpose of identifying prenatal drug exposure, it also finds widespread application in the early identification of cystic fibrosis, identifying prenatal exposure to neurotoxicants such as organic pesticides, detection of cytomegalovirus, and as an indicator of the mineral nutritional history of the fetus [66, 97-99]. 


\section{Chapter 2: Introduction to the Laboratory Analysis of Toxicological Specimens}

\subsection{Introduction}

The analytical methodology employed for the toxicological analysis of biological specimens depends largely on whether the testing aims to provide qualitative or quantitative information. Although the specifics of testing procedures may vary slightly from laboratory to laboratory, the process of measurement is usually divided into an initial screening test followed by a confirmation test. Most laboratories perform an initial screening test when asked to analyze any specimen for the presence of a certain drug or drug class. Screening tests provide the analyst with a relatively quick and cost effective way to determine whether a drug or drug class is likely to be present in a given specimen. Screening tests may be designed to detect the presence of a certain drug class, such as benzodiazepines, or may be a broad drug screen performed on a GC/MS which enables the analyst to screen for many drugs based on retention times and mass spectral data. Following a positive screen result, the presence of a drug or drug class should be confirmed using a second technique which draws on a different chemical principle. Confirmatory tests should be more selective for the target analyte than the initial screen.

Forensic toxicology laboratories routinely offer specific testing panels aimed at detecting and quantifying specific drugs such as 'drugs of abuse', 'analgesic medications', 'drug facilitated sexual assault (DFSA) drugs', or 'drugs capable of causing impairment'. Obviously the total number of drugs encountered in any one forensic case can be large and while certain drugs will provide important toxicological information, many of the drugs will not be relevant to the case at hand. By offering panels consisting of selected analytes, forensic toxicology laboratories can 
provide the most relevant information without the cost of testing for additional analytes with little significant to the case.

Confirmation assays are designed to identify and quantify only target analytes. The number of analytes included in each confirmation assay is often less than the number of compounds capable of generating a positive screen. Confirmation assays are generally designed to identify and quantify all analytes included in a specific panel. While positive screening results are possible due to cross reacting interferences, positive confirmations only arise when one or more target analytes are present in the sample. Table 1 outlines the common analytes and testing procedures for a typical 'drugs of abuse' testing panel. 
Table 1: Common analytes detected during 'drugs of abuse' screening and confirmation analysis.

\begin{tabular}{|c|c|c|}
\hline Drug class & Analytes detected in screen testing & $\begin{array}{l}\text { Analytes detected in } \\
\text { confirmation testing }\end{array}$ \\
\hline Amphetamines & $\begin{array}{l}\text { - Amphetamine derivatives } \\
\text { - Cross reactants (ranitidine, bupropion) }\end{array}$ & $\begin{array}{l}\text { - Amphetamine } \\
\text { - Methamphetamine } \\
\text { - MDMA }\end{array}$ \\
\hline Cannabinoids & $\begin{array}{l}\text { - Cannabinoids (THC and metabolites) } \\
\text { - Cross reactants (pantoprazole, omeprazole) }\end{array}$ & $\begin{array}{l}\cdot \text { TCH } \\
\cdot \text { THC-COOH } \\
\end{array}$ \\
\hline Cocaine & $\begin{array}{l}\text { - Cocaine and metabolites } \\
\text { - Cross reactants }\end{array}$ & $\begin{array}{l}- \text { - Cocaine } \\
\text { - Benzoylecgonine } \\
\text { - Cocaethylene }\end{array}$ \\
\hline Methadone & $\begin{array}{l}\text { - Methadone and metabolites } \\
\text { - Cross reactants (doxylamine) }\end{array}$ & $\begin{array}{l}\cdot \text { Methadone } \\
\cdot \text { EDDP }\end{array}$ \\
\hline Barbiturates & $\begin{array}{l}\text { - Barbiturates and metabolites } \\
\text { - Cross reactants (Phenytoin) }\end{array}$ & $\begin{array}{l}\text { - Butalbital } \\
\text { - Phenobarbital } \\
\text { - Pentobarbital } \\
\text { - Amobarbital } \\
\text { - Butabarbital }\end{array}$ \\
\hline Benzodiazepines & $\begin{array}{l}\text { - Benzodiazepines and metabolites } \\
\text { - Cross reactants (oxaprozin, sertraline) }\end{array}$ & $\begin{array}{l}\text { - Diazepam } \\
\text { - Nordiazepam } \\
\text { - Clonazepam } \\
\text { - Temazepam } \\
\text { - Alprazolam }\end{array}$ \\
\hline Opiates & $\begin{array}{l}\text { - Opiate derivatives } \\
\text { - Cross reactants (fluoroquinolones, ofloxacin) }\end{array}$ & $\begin{array}{l}\text { - Morphine } \\
\text { - Codeine } \\
\text { - Hydrocodone } \\
\text { - Hydromorphone } \\
\text { - Oxycodone } \\
\text { - Oxymorphone } \\
\text { - 6-monoacetylmorphine }\end{array}$ \\
\hline
\end{tabular}

Mass spectrometry (MS) has long been the recommended technique for confirmatory testing and should be employed where possible and practical. Mass spectrometry provides the analyst with unique structural information making the unequivocal identification of individual drugs possible, even in the presence of chemically similar compounds. Confirmation analysis employing mass spectrometry is generally performed following a positive immunoassay screen. Both instrumentation and sample preparation procedures required for mass spectrometric analysis can be cost ineffective. Initial presumptive screening employing more cost effective 
immunoassay techniques identifies a large majority of negative samples. This reduces the number of required confirmations and therefore the overall cost of analysis.

Mass spectrometers coupled to either gas chromatographic (GC/MS) or liquid chromatographic (LC/MS) systems are the most commonly employed analytical techniques for confirmatory testing in the toxicology laboratory. Gas chromatography coupled with mass spectrometry has long been the most commonly employed confirmation technique in the toxicology laboratory. In recent years however, the coupling of liquid chromatography with mass spectrometry has found widespread use due to its decreased sample preparation requirements and increased sensitivity. Liquid chromatography also alleviates thermal complications associated with GC analysis. Many compounds do not naturally lend themselves to GC analysis as they are either too polar or insufficiently volatile. By chemically derivatizing the target analyte to either increase its overall volatility or decrease its polarity, it is possible to increase the suitability of a certain compound for GC analysis. While chemical derivatization alleviates some of the thermal complications associated with GC analysis, sample preparation requirements and long run times can prove time and cost ineffective in high throughput toxicology laboratories. Thermal stability also presents a problem when analyzing small drug molecules, as thermally labile compounds may undergo degradation in the injection port of the GC.

Liquid chromatography offers the analyst the advantage of introducing samples in aqueous solvents and eliminates the need for chemical derivatization, thus greatly reducing sample preparation time and cost. Liquid chromatography employing tandem mass spectrometry has recently become the preferred technique for toxicological analyses due to its superior selectivity and sensitivity which enables the analyst to quantify lower levels of analyte in more complex matrices with reduced sample preparation. 


\subsection{Sample Preparation Techniques}

Sample preparation refers to a series of steps which are performed to transform a sample into a form that is suitable for analysis. There are numerous ways to prepare samples for chemical analysis and the method of choice depends on the nature of the sample, the method of chemical analysis that will follow preparation, and whether the testing is presumptive or confirmatory. Sample analysis in the forensic toxicology laboratory is typically chromatographic which requires more extensive sample cleanup than immunoassay or spectrophotometric based techniques.

Due to the complex nature of biological specimens and the low concentrations of drug commonly encountered in forensic analysis, analytes of interest are often isolated or extracted from the sample matrix and concentrated prior to instrumental analysis[100]. Direct chromatographic analysis of biological specimens is not practical due to the large number of naturally occurring compounds present in specimens such as whole blood, urine, and meconium. Biological specimens are so rich in endogenous interfering species that any attempts to identify or quantify low levels of xenobiotics without prior isolation would prove inaccurate. Isolation and concentration of an analyte from a biological matrix prior to chromatographic analysis allows for a more accurate identification/quantitation and ensures instrument longevity.

Chemical extractions, for the purpose of isolating and concentrating an analyte of interest from a biological matrix, are most commonly achieved using either liquid-liquid extractions (LLE) or solid-phase extractions (SPE). 


\subsubsection{Liquid - Liquid Extraction (LLE)}

Liquid-liquid extractions utilize differences in the $\mathrm{pH}$ and solubility characteristics of an analyte to effectively remove the compound from an aqueous matrix such as blood or urine. Liquid-liquid extractions are achieved by introducing an immiscible organic solvent to an appropriately buffered aqueous specimen. Compounds in a non-ionized form will prefer the lipophilic environment of the organic solvent while ionized species will remain the aqueous environment of the biological sample. By appropriately buffering the biological sample according the $\mathrm{pH}$ characteristics of the analyte, and introducing an immiscible organic solvent, un-ionized drug molecules will transfer to the organic layer while many of the biological components such as proteins remain in the aqueous layer. It is on this basis that liquid-liquid extractions allow for the effective removal of analytes from the surrounding biological matrix[100].

Developing an efficient LLE requires knowledge of the physicochemical properties of the target analyte. Basic compounds are routinely extracted into organic solvents by first buffering the sample with an appropriate base or basic buffer solution. Under alkaline conditions, basic compounds will be unionized and will readily transfer into the organic environment of solvents such as hexane, toluene, dichloromethane or chloroform. Similarly, acidic analytes may be extracted into such solvents by first buffering the aqueous sample with an acid or acidic buffer solution to ensure that the target analyte is present in an un-ionized form. Target analytes are generally extracted into organic solvents with densities less than that of the aqueous layer to allow for easy transfer and subsequent concentration of the analyte through simple dry down and reconstitution steps. 


\subsubsection{Solid Phase Extractions (SPE)}

Solid phase extractions also aim to isolate and concentrate target analytes by removing sample impurities. Solid-phase extractions (SPE) were originally developed for the purpose of alleviating some of the time-related problems associated with multi-step liquid-liquid extractions[100]. In SPE, appropriately buffered samples are applied to a silica gel based packing material which is contained within a syringe barrel cartridge. Extractions are based on the physicochemical interaction between the packing material and the target analyte.

Sample preparation using SPE usually consists of four basic steps; which include column conditioning, sample loading, wash steps, and analyte elution. The first step of a solid phase extraction is referred to as column conditioning which aims to make the subsequent extraction easier and more efficient. Conditioning the column with a non-polar solvent not only removes any impurities in the cartridge but also ensures maximum contact of the liquid and solid phases. Conditioning of the column with a solvent similar in nature to the sample solvent ensures that the sample will experience similar $\mathrm{pH}$ conditions when loaded onto the column. Failure to condition the column can result in poor recoveries and irreproducible results[101].

Following column conditioning, the sample is loaded onto the SPE column in a solvent that is weak for the sorbent being used[101]. If the solvent is too strong for the analyte of interest, the analyte will not be retained on the sorbent and will simply pass through the column into waste. Once the sample has been loaded onto the column, several wash steps are generally performed to wash all remaining sample onto the sorbent and remove any undesirable sample components. Wash steps are generally performed with a weak solvent similar in nature to the loading solvent to ensure that no analyte is eluted during the wash process. Analyte elution is the final step of SPE and is achieved using a solvent that is strong for the sorbent being used. 
While all SPE are performed using a similar sequence of events as the ones described above, the nature of the packing material may be varied to provide different retention mechanisms. Normal phase SPE involves the use of a solid phase consisting of polar functionally bonded silica with short carbon chains. In normal phase SPE, the polar sorbent acts to retain polar analytes while less polar components are washed through the sorbent into waste. Analyte elution is then achieved with a highly polar solvent. Reversed phase SPE utilizes a non-polar solid sorbent which generally consists of silicon derivatized with hydrocarbon chains. Retention is based on the hydrophobic effect and only non-polar or weakly polar analytes will retain on the sorbent surface. Analyte elution is achieved using non-polar organic solvents which disrupt the hydrophobic interaction between the sorbent and the analyte.

While normal phase and reversed phase SPE separate analytes based on polarity, ionexchange SPE separates analyte based on electrostatic interaction existing between a charged or ionized analyte and a charged sorbent. Ion-exchange SPE requires that both the analyte of interest and the functional groups bonded to the solid phase are charged. Special consideration must therefore be given to the solvents of choice and their respective $\mathrm{pH}$.

Anion exchange sorbents are derivatized with positively charged functional groups and act to retain negatively charged analytes. Anion exchange SPE is well suited for the extraction of acidic compounds following buffering with a base to ensure that the analyte is negatively charged. Sample elution is performed through the addition of an organic solvent containing a concentrated acid $(1-2 \% \mathrm{v} / \mathrm{v})$ which acts to neutralize the molecule and increase its solubility in the organic elution solvent. 
Cation exchange sorbents are derivatized with negatively charged functional groups and act to retain positively charged analytes. Cation exchange SPE is well suited for the extraction of basic compounds following buffering with an acid to ensure that the analyte is positively charged. Sample elution is performed through the addition of an organic solvent containing a concentrated base $(1-2 \% \mathrm{v} / \mathrm{v})$ which acts to neutralize the molecule and increase its solubility in the organic elution solvent.

\subsection{Chromatographic Separations in Forensic Toxicology}

The term chromatography defines the analytical separation technique in which the components of a mixture are carried through a stationary phase by the flow of a mobile phase. Subsequent separation of the individual components is based on the differences in migration rates among the sample components[102]. The two general categories of chromatography most commonly employed in forensic toxicology are liquid chromatography (LC) and gas chromatography (GC). In the former, the mobile phase is a liquid; in the latter it is a gas. Chromatographic separations play an integral role in forensic toxicology as traditional toxicological specimens commonly encountered in post mortem analysis require extensive sample separation to isolate the compound of interest from matrix interferences and other drugs.

Gas chromatographic separations are performed by vaporizing and injecting a small volume of sample (typically $1 \mu \mathrm{L}$ ) onto the head of a chromatographic column. The sample components are swept along the stationary phase by an inert carrier gas such as helium and the differing degrees of interaction between the gaseous analytes and the stationary phase causes the individual sample components to elute at different times. Stationary phases are comprised of a microscopic layer of liquid or polymer coated onto an inert solid support and contained within a metal capillary column maintained in a temperature-regulated oven[103]. Following injection of 
a sample mixture onto the chromatographic column, each component partitions between the stationary phase and the mobile phase as it is continuously swept toward the detector. Sample components that have a high affinity for the stationary phase spend more time in that phase and take longer to reach the detector. As the individual components begin to elute from the chromatographic column, the detector produces a signal that is proportional to the amount of substance that passed through it[103]. Individual components that elute from the column are identified by a characteristic retention time which is a reproducible measure of the time interval between sample injection onto the column and peak detector response. Gas chromatographs are commonly interfaced with mass spectrometers because the low flow rates employed for capillary GC allow the column eluent to be directly fed into the ionization chamber of the mass spectrometer making for easy coupling.

Liquid chromatographic separations are achieved by injecting a small volume of sample onto a column which is tightly packed with stationary phase particles. Sample components are carried through the column by a continuous flow of a liquid mobile phase solution. Individual compounds pass through the column at different rates due to the differences in their partitioning behavior between the mobile liquid phase and the stationary phase. Unlike gas chromatographic separations where there is no direct interaction between the mobile phase (carrier gas) and the analyte, each sample component experiences differing degrees of both chemical and physical interactions with the stationary phase and mobile phase as it traverses the LC column. Analytes are identified by a characteristic retention time which is an accurate measure of the time interval between sample injection onto the column and peak detector response.

Before the development and implementation of sophisticated LC/MS interfaces, high performance liquid chromatography (HPLC) was the fastest growing and most widely employed 
analytical separation technique due to its suitability for separating nonvolatile or thermally labile species not amendable to GC. Traditional HPLC systems were coupled to one of several detection systems that proved compatible with the liquid phase separation technique such as refractive index detectors, UV/VIS (fixed wavelength, variable wavelength, diode array) detectors, fluorescence detectors, and electrochemical detectors. The successful coupling of liquid chromatography to mass spectrometry required the development of sophisticated interfaces capable of combining the liquid phase separation technique, with the gas phase mass spectrometry technique carried out under vacuum.

\subsection{Ultra Performance Liquid Chromatography (UPLC)}

Ultra performance liquid chromatography (UPLC) is an emerging analytical technique which draws upon the principles of chromatography to run separations at higher flow rates for increased speed, while simultaneously achieving superior resolution and sensitivity. Ultra performance liquid chromatography was developed through the recognition that a reduction in the stationary phase particle size will have the greatest benefit to any chromatographic process[104-106]. While the increased efficiency of small particle chromatography has long been recognized, development of techniques utilizing this science is complicated by the large increases in system backpressures encountered when pumping mobile phase through sub $2 \mu \mathrm{m}$ particles. Traditional HPLC systems are unable to operate at backpressures typically afforded by small particle chromatography. While there is no single separation parameter that distinguishes between 'high performance' and 'ultra performance' liquid chromatography, UPLC refers to chromatographic separations employing sub $2 \mu \mathrm{m}$ stationary phase particles of high mechanical strength. 
The evolution of packing materials designed and employed to directly affect chromatographic separations has been based on the underlying principles of the van Deemter equation (Equation 1)[104] [107].

$$
\mathrm{H}=\mathrm{A}+\mathrm{B} / \mu+\mathrm{C} \mu
$$

Equation 1

The van Deemter equation is an empirical formula that describes the relationship between mobile linear velocity $(\mu)$ and column efficiency (HETP or H) (Equation 1)[104]. The van Deemter plot (Figure 1) illustrates the principles of the van Deemter equation and is used to predict and determine the mobile phase flow rate where column efficiencies will be maximized[108].

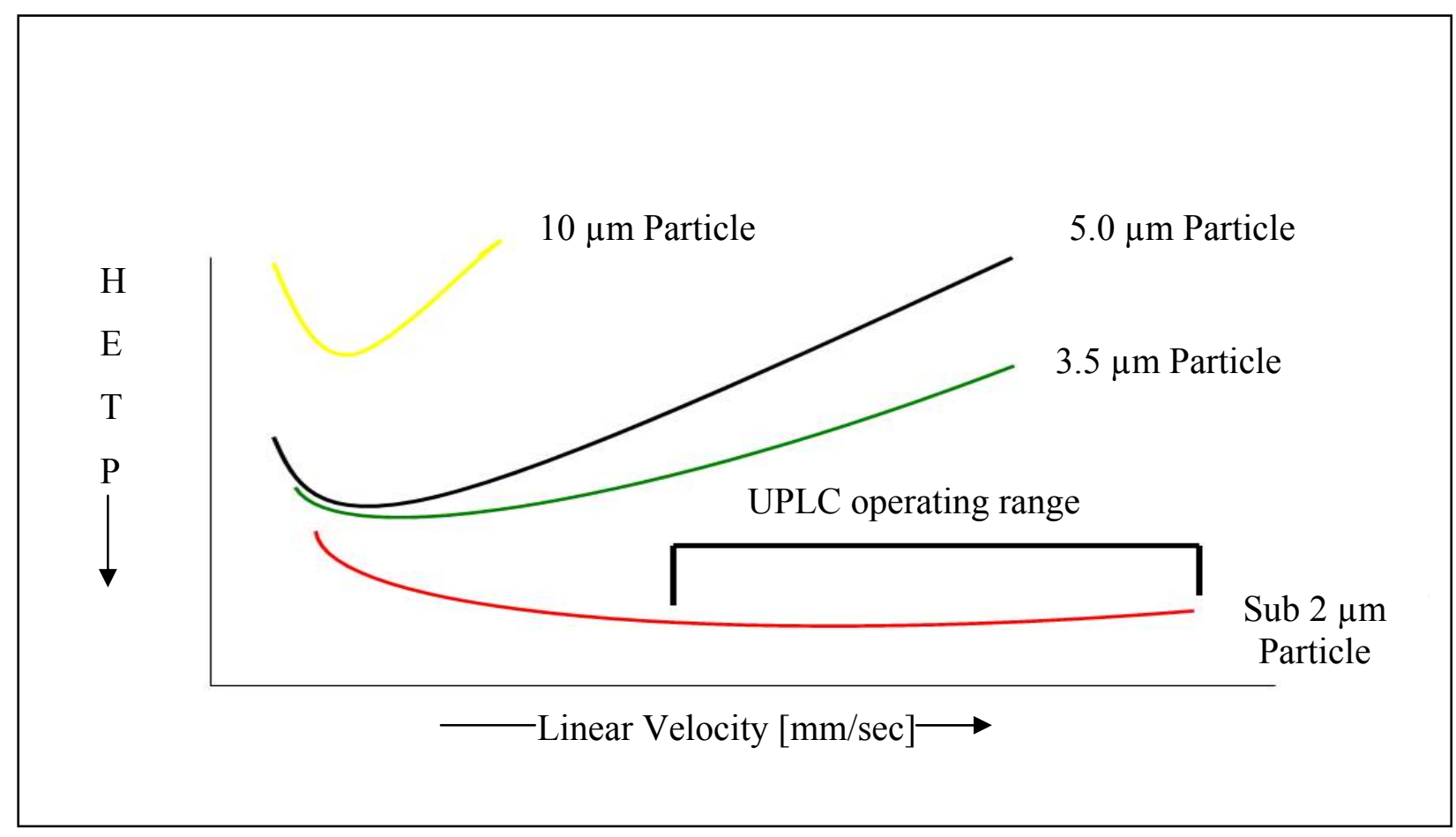

Figure 1: Van Deemter plot (Adapted from Waters, 2004). 
Figure 1 illustrates how particle diameter can significantly reduce the HETP resulting in higher separation efficiencies. The extended minimum of the sub $2 \mu \mathrm{m}$ particles seen in Figure 1 indicates that increases in mobile phase flow rates do not have the same negative influences on separation efficiency as seen with the larger particles. This means that increased efficiencies are available over a much wider range of flow rates and the speed of analysis can be increased without sacrificing efficiency or resolution[104, 106].

Column efficiency is used to evaluate the performance of a stationary phase through its ability to accomplish particular separations. Such evaluation entails how well the column is packed and its kinetic performance. Column efficiency is commonly measured by the number of theoretical plates (N) in a stationary phase. This measure of column efficiency is easily calculated by obtaining an analytes retention time (tR) and peak standard deviation $(\sigma)$ from a chromatogram and substituting the values into Equation 2.

$$
\mathrm{N}=(\mathrm{tR} / \sigma)^{2}
$$

Equation 2

The more theoretical plates a column has, the more efficient it is deemed. Although plate number is still an accurate way to determine column efficiency, a more appropriate parameter for measuring efficiency is the plate height or the height equivalent to a theoretical plate (HETP) which is calculated from the column length (L) and the number of theoretical plates $(\mathrm{N})$ (Equation 3).

$$
\mathrm{H}=\mathrm{L} / \mathrm{N}
$$
Equation 3

Measuring column efficiency based on the height equivalent to a theoretical plate (HETP) allows for the normalization of plate number for columns of different lengths. Plate height is traditionally measured in millimeters and as plate height decreases, column efficiency increases 
as there will be more equilibrations along the column length, leading to a more efficient separation.

Efficiency is the primary separation parameter behind UPLC since it relies on the same selectivity and retentivity as HPLC[107]. Review of the fundamental resolution (Rs) equation (Equation 4) reveals that chromatographic resolution is directly proportional to the square root of column efficiency.

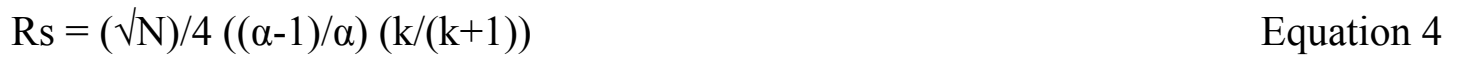

Increasing column efficiency will not only result in greater resolving power but also gives rise to narrower peaks due to the fact that column efficiency is inversely proportional to the square root of the peak width (Equation 5).

$$
\mathrm{N} \alpha 1 / \mathrm{w}^{2}
$$

Equation 5

Narrower peak width would be expected to contribute to increased resolving power because narrower peaks are easier to separate[107]. Increased efficiency also leads to an increase in sensitivity, according to Equation 6, which states that peak height, and therefore sensitivity, is inversely proportional to peak width (Equation 6).

$\mathrm{H} \alpha 1 / \mathrm{w}$ Equation 6

Yet another benefit to the chromatographer is the concomitant increase in peak capacity per unit time in gradient separations that accompanies the taller, narrower peaks[105]. Recognition that increases in column efficiency lead to subsequent increases in resolution and sensitivity has long existed and as a result analysts commonly employ various methods to maximize column efficiency. According to Equations 2 and 3, gains in column efficiency can be 
achieved by increasing the number of theoretical plates or by reducing the height of the theoretical plates. Increasing the number of theoretical plates through the employment of longer columns may not always be desirable as this leads to increases in retention time and band broadening. This especially holds true for high throughput forensic toxicological analysis where sample run time must be minimized to minimize sample turnaround time, and losses in sensitivity through band broadening are not affordable due to the already low concentrations of analyte present in typical samples. As a result, increases in column efficiency are more commonly achieved through the reduction of theoretical plate height.

The van Deemter equation describes the relationship between linear flow velocity $(\mu)$ and column efficiency $(\mathrm{H})$, where $\mathrm{A}, \mathrm{B}$, and $\mathrm{C}$ are constants related to the mechanistic components of band broadening (Equation 1). Maximizing separation efficiencies through the reduction of theoretical plate height $(\mathrm{H})$ requires a thorough understanding of the van Deemter equation and its individual components which all contribute to band broadening.

Eddie diffusion, also known as multiple flow path diffusion, is a phenomenon which contributes to zone spreading and is represented by the constant ' $\mathrm{A}$ ' in the van Deemter equation. When a mobile phase moves through a porous medium such as a packed column, parts of the stream take a more tortuous path than others. Zone spreading is the result because some solute molecules take longer paths and lag behind the average, while others take shorter paths and move ahead of the average[109]. Longitudinal diffusion is another phenomenon which contributes to band broadening and can is represented by the constant ' $\mathrm{B}$ ' in the van Deemter equation. When solutes are contained in a fluid such as a mobile phase stream, they will naturally diffuse and spread based on the concentration gradient that exists. Regardless of how discrete the solute band, once in the mobile phase, solutes will diffuse from the center of the band to more dilute 
regions resulting in zone spreading. Due to the fact that diffusion coefficients of mobile phases are orders of magnitude smaller in liquids than they are in gases, the longitudinal diffusion constant $(\mathrm{B} / \mu)$ approaches zero in the van Deemter equation as it is directly proportional to the mobile phase diffusion coefficient. Mass transfer processes that occur in the mobile phase also result in zone broadening and this phenomenon is represented by the constant ' $\mathrm{C}$ ' in the van Deemter equation. Such broadening occurs because both the flowing stream of the mobile phase, and the stationary phase particles have finite widths and time is required for solute molecules to diffuse from the interior of these phases to the interfaces which is where transfer can occur. As a result, analyte molecules at the front of the band are swept ahead before they have time to equilibrate with the stationary phase and molecules at the trailing edge of the band are left behind by fast moving mobile phase[109]. Table 2 outlines the components of the Van deemter equation and their relationship to column and analyte properties.

Table 2: Components of the Van deemter equation and their relationship to column and analyte properties

\begin{tabular}{|c|c|c|}
\hline Process & Term in equation & $\begin{array}{c}\text { Relationship to column and analyte } \\
\text { properties }\end{array}$ \\
\hline Eddie diffusion & $\mathrm{A}$ & $\mathrm{A}=2 \lambda \mathrm{dp}$ \\
\hline Longitudinal diffusion & $\mathrm{B} / \mu$ & $\mathrm{B} / \mu=\left(2 \gamma \mathrm{D}_{\mathrm{M}}\right) / \mu$ \\
\hline Mass transfer in mobile phase & $\mathrm{C}_{\mathrm{M}} \mu$ & $\mathrm{C}_{\mathrm{M}} \mu=\left(\mathrm{f}_{\mathrm{M}}\left(\mathrm{k}^{\prime}\right) \mathrm{d}^{2}{ }_{\mathrm{p}} \mu\right) / \mathrm{D}_{\mathrm{M}}$ \\
\hline
\end{tabular}

Careful examination of the relationships existing between the three diffusion phenomena and the column properties, leads to the conclusion that a reduction in the diameter of stationary phase particles will have the greatest impact on theoretical plate height, and therefore separation efficiency. Zone spreading, as a result of Eddie diffusion and mass transfer effects, is directly proportional to stationary phase particle diameter. Reducing the diameter of the stationary phase 
particle will maximize separation efficiency through the reduction of Eddy diffusion and diffusion resulting from mass transfer effects. Although stationary phase particle diameter does not directly affect the phenomena of longitudinal diffusion, the higher linear velocities affordable with smaller particles (see below) act to minimize longitudinal diffusion effects and further maximize separation efficiency.

Reducing stationary phase particle size has been exploited as the driving force for increasing separation efficiencies for more than forty years, however until the introduction of UPLC, the scope of this technique was somewhat limited. Not only are traditional HPLC system designs unable to operate at the high backpressures associated with small particle chromatography, but traditional detection systems lack the speed required to generate sufficient data when working with peaks that may only be a few seconds wide[107].

Examination of some fundamental chromatographic equations helps to illustrate the new levels of speed, sensitivity and resolution provided by the three fold decrease in particle size from $5 \mu \mathrm{m}$ (HPLC scale) to $1.7 \mu \mathrm{m}$ (UPLC scale). According to the van Deemter plot, (Figure 1) column efficiency $(\mathrm{N})$ is inversely proportional to particle size (dp) (Equation 7) due to decreased zone broadening with smaller particles.

$\mathrm{N} \propto 1 / \mathrm{dp}$ Equation 7

As a result, a decrease in particle size from $5 \mu \mathrm{m}$ to $1.7 \mu \mathrm{m}$ will result in a threefold increase in efficiency. The fundamental resolution equation (Equation 4) tells us that resolution is proportional to the square root of efficiency meaning that the threefold increase in efficiency afforded by the $1.7 \mu \mathrm{m}$ particles also provides a $70 \%$ increase in resolution. Efficiency is also inversely proportional to the square root of peak width (Equation 5) meaning that peak widths 
will be $70 \%$ narrower as a result of the reduced particle size. A $70 \%$ reduction in peak width results in a 70\% increase in peak height and therefore sensitivity. Reduced peak width also provide the chromatographer with a greater peak capacity per unit time in gradient separations[107]. Smaller particles also enable extraordinary increases in analysis speed without sacrificing resolution[110]. According to Equation 8 column length can be reduced proportionally as particle size is decreased without losing efficiency[110].

$$
\mathrm{N}=\mathrm{L} / \mathrm{dp}
$$

Equation 8

This enables the chromatographer to perform separations in one third of the analysis time. Moreover, van Deemter theory states that the optimum flow rate (Fopt), corresponding to maximum separation efficiencies, increases as particle size decreases, according to Equation 9.

Fopt $\alpha 1 / \mathrm{dp}$ Equation 9

As can be seen from the van Deemter plot (Figure 1), as particle size decreases, the corresponding HETP also decreases, resulting in higher efficiencies. It is also evident from the van Deemter plot that the highest efficiencies are available over a much wider range of flow rates with smaller particles than with larger particles.

The separation efficiency of HPLC increased as the particle size of column packing decreased from $10 \mu \mathrm{m}$ in the $1970 \mathrm{~s}$ down to $3.5 \mu \mathrm{m}$ in the $1990 \mathrm{~s}[111]$. One consistent observation with all of these particles sizes, as well as the $2.5 \mu \mathrm{m}$ particles used in the early 2000s was that HETP decreased to a minimum value and then increased with increasing flow rate[111] (Figure 1). When employing $1.7 \mu \mathrm{m}$ stationary phase particles such as those used in UPLC, the resulting van Deemter plot not only exhibits a decreased HETP relative to the larger particles but also offers an extended minimum over a wider range of linear velocities[104, 111]. 
As a result, flow rate or speed of analysis can be optimized without sacrificing resolution. This means that when transitioning from $5 \mu \mathrm{m}$ to $1.7 \mu \mathrm{m}$ particles, not only can column length be reduced by a factor of 3 , but the separation can be run at three times the flow rate. This translates to a nine fold increase in throughput with no loss in efficiency or resolution[110].

Since backpressure is proportional to flow rate, achieving small particle, high peak capacity separations requires fully redesigned HPLC systems capable of operating at backpressures beyond the capabilities of today's system designs[112]. To take full advantage of the increased speed, superior resolution and sensitivity afforded by smaller particles, instrument technology had to be fully redesigned[112].

To fully realize the potential speed, sensitivity and resolution of UPLC separations, new pressure - tolerant reversed phase particle had to be developed. Production of extremely small, efficient particles with high mechanical strength would allow the analyst to surpass the performance standards of current HPLC column technology. Early investigations into the use of sub $2 \mu \mathrm{m}$ particles for ultrahigh-pressure reversed phase chromatography were reported by MacNair and coworkers who employed $1.5 \mu \mathrm{m}$ nonporous octadecyl-silane-modified silica particles to achieve theoretical plate counts as high as 300,000 for lightly retained compounds[113]. In more recent years, Waters Corporation has utilized a bridged ethylsiloxane/silica hybrid (BEH) structure with a narrow particle size distribution, produced by the condensation of 1,2-bis(triethoxysilyl)ethane and tetraethoxysilane[114]. This new hybrid material was developed in a $1.7 \mu \mathrm{m}$ particle to improve efficiency, ruggedness, $\mathrm{pH}$ range, peak shape and loading capacity, as well as the ability to run at elevated backpressures and temperatures $[112,114]$. The interconnection of silica atoms with ethyl groups whilst maintaining a silica backbone has proven to be a key success factor for Waters UPLC columns because it 
means maintaining the strength of silica, while achieving reduced silanol activity and improved resistance to alkaline conditions[114]. Peak shape is further optimized using trifunctional C18 bonding chemistry and a proprietary end-capping procedure[114].

High efficiency separations employing $1.7 \mu \mathrm{m}$ solid phase particles routinely produce peaks with a half-height width of less than 1 second which poses significant challenges for the detection system[110]. One major concern when operating with very narrow peaks is the ability of the mass spectrometer to obtain a sufficient number of data points across a peak to perform peak integration and data-dependant MS/MS analysis[115]. To accurately and reproducibly quantify analyte peaks with half-height widths of less than one second, detection systems capable of rapid data acquisition are required to ensure that sampling rates are high enough to capture sufficient data points across such narrow peaks.

\subsection{Mass Spectrometry (MS)}

Mass spectrometry describes the analytical technique in which components of a sample or mixture are converted into rapidly moving gaseous ions and subsequently separated and identified based on their mass-to-charge ratios. Mass spectrometry provides both qualitative (structural) and quantitative (molecular mass or concentration) information about analyte molecules following their conversion into gaseous ions[116]. Mass spectrometry is typically preceded by a chromatographic separation such as gas chromatography (GC) or liquid chromatography (LC) to resolve the molecules of interest from possible matrix interferences. Following elution from the chromatographic column, molecules of interest are introduced into the ionization source of the mass spectrometer. 
Ionization can be achieved using a variety of mechanisms which are referred to as either "hard" or "soft" ionization techniques as described my McLafferty et al[117]. Hard ionization techniques produce ions which possess appreciable internal energies and tend to undergo fragmentation, whereas soft ionization techniques produce ions with low internal energy which reduces the propensity for fragmentation. Efficient ionization produces either positive or negative ions of interest which then travel through the mass analyzer where they are separated and/or filtered according to their mass-to-charge $(\mathrm{m} / \mathrm{z})$ ratio. Following mass analysis, stable ions make contact with the detector where a signal is generated describing the relative abundance of each signal according to the mass-to-charge ratio[116].

Mass spectrometry is recommended as the confirmatory technique in forensic toxicological analysis because of its ability to generate a unique mass spectrum for most compounds which can be used to characterize the sample[118]. Strong recommendations for the use of mass spectrometry exist because of its ability to both determine molecular weight and elucidate structural information[119].

Although direct mass spectrometric analysis is feasible through injection without prior chromatographic separation, traditional toxicological analyses have utilized mass spectrometers coupled to gas chromatographs. Chromatographic separations are often required prior to mass spectrometric analysis due to the complex biological matrices typically encountered in the forensic toxicology laboratory. Due to the ease at which gas chromatographic systems are interfaced with mass spectrometers, GC/MS analysis has become the most common technique for toxicological screening and confirmation in the forensic and clinical industries. 
Electron ionization (EI) is a hard ionization technique commonly employed in GC/MS experiments. EI induces significant fragmentation of the analyte giving rise to characteristic fragments which are subsequently used for structural information. The masses of these fragments are characteristic of the original analyte molecule and the way in which it fragmented under EI conditions. Confirmation analysis by GC/MS has traditionally offered superior sensitivity, selectivity, speed and precision and remains the method of choice for the detection and quantification of drugs and poisons volatile under GC/MS conditions[120]. GC/MS is still widely employed in many toxicology laboratories for a number of reason including; (1) instrumentation is readily available and reasonably inexpensive, (2) laboratory staff have extensive working knowledge of both the hardware and software, (3) electron impact ionization (EI) provides structural information through 'hard' ionization, (4) extensive searchable mass spectral libraries exist to assist with identification of unknowns, and (5) GC/MS interfaces are relatively simple and easy to maintain[121].

While gas chromatographs represent an almost ideal inlet device for a mass spectrometer, the chromatographic separation itself is not suited to all molecules and hence limits both the capabilities and applications of GC/MS in the toxicology laboratory. In order for a compound to be amendable to GC analysis it must possess sufficient volatility so that its molecules exist in the gas or vapor phase at temperatures at or below $400^{\circ} \mathrm{C}$. Gaseous analyte molecules need also be thermally stable to avoid sample degradation in the injection port of the gas chromatograph. Many drugs of forensic importance such as the benzodiazepines, tricyclic antidepressants, and acetaminophen are not naturally amendable to $\mathrm{GC}$ as a result of their high polarity, thermal lability or low volatility[122]. As a result LC has acquired a role of growing importance due to its separation capabilities for such compounds[123]. 
Early in development, conventional detection systems for LC did not include mass spectrometry. This was because solutes eluting from an LC column are dissolved in a liquid mobile phase at atmospheric pressure, and mass spectrometers are configured to detect gas phase ions under vacuum. Due to the incompatibility of LC with MS detection, unequivocal identification of analytes was not always possible due to the possibility of co-elution and falsepositives associated with ultra violet (UV) detection. While the potential capabilities and advantages of coupling LC with mass spectrometry (LC/MS) were well recognized, design and development of sophisticated interfaces for devices having different strategies proved both time consuming and expensive.

\subsection{The Rise of Liquid Chromatography - Mass Spectrometry (LC/MS)}

Research and long-term development over the last 35 years has produced seven major interfacing techniques that have made LC/MS experiments both possible and suitable for the field of analytical and forensic toxicology[124]. In 1969 Tal'roze and coworkers employed a capillary inlet interface in one of the earliest reported attempts at developing an LC/MS experiment[125]. Since the early work of Tal'roze et al., various interfaces have been designed to achieve both chromatographic solvent evaporation and analyte ionization. Elimination of the chromatographic solvent prior to MS detection, flow rate incompatibilities, the inability of mass spectrometers to handle non-volatile buffers, and achieving efficient and reproducible ionization

of non-volatile and/or thermally labile analytes all arose as problematic complications during the development of LC/MS interfaces. Several interfaces, each providing a variety of technological solutions to the problems listed above, have transformed LC/MS into a robust, widely applicable and wide-spread analytical technique[126]. 


\subsection{LC/MS Interfaces}

Recent reviews have indicated that of the 25 different LC/MS interfaces that have been described in the literature over the past 20 years, only 5 are actively used today and even fewer are the subject of any further developmental research efforts[127, 128]. Interfaces most commonly employed today include thermospray (TS), particle beam (PB), fast atom bombardment (FAB), atmospheric pressure chemical ionization (APCI), and electrospray ionization (ESI).

\subsubsection{Thermospray Interface (TP)}

The original thermospray interface introduced by Vestal and co-workers in 1983 used a heated vaporizer tube to generate a jet of vapor and small droplets from the LC column effluent[129, 130]. The pressurized solution flows through the heated capillary at flow rates approaching $2 \mathrm{~mL} / \mathrm{min}$ where it undergoes almost complete volatilization. It is then nebulized into an expansion chamber where it is ionized at low pressure by the solvent buffer (solventmediated chemical ionization), a filament, or by a discharge electrode[124].

Following ionization and complete desolvation, analyte ions are transferred to the mass spectrometer by means of a repeller electrode which can have varying voltages applied to it to enhance fragmentation of the analyte. Application of a higher voltage to the repeller electrode increases the acceleration of the analyte ions so that subsequent collisions with residual solvent molecules will result in fragmentation.

Mass spectra resulting from thermospray ionization usually yield little structural information and prove less useful for the identification of unknown compounds. Because of this, TS ionization is generally employed for target compound analysis[130]. A review in 1997 
highlighted the widespread use of the thermospray interface in the early 1990s, in particular, for the sensitive detection of steroids, drugs of abuse, thiourea pesticides, mycotoxins, indolic compounds and tricyclic antidepressants[124]. The introduction of more sensitive and robust approaches to mass spectrometric analyses based on atmospheric-pressure ionization techniques have largely replaced thermospray applications[131].

\subsubsection{Particle Beam Interface (PB)}

The original particle beam (PB) interface was introduced as the 'mono-disperse aerosol generating interface for chromatography' (MAGIC-LC-MS) in 1984 by Willoughby and Browner[132]. In PB ionization experiments, the chromatography effluent is nebulized either pneumatically or by thermospray nebulization at atmospheric pressure into a slightly heated desolvation chamber. The desolvation chamber is connected to a momentum separator which is the principle component of the PB interface. Following desolvation, analytes are transferred into the low-pressure ion source through a two-stage jet separator where the high mass analytes are preferentially transferred to the MS ion source while the low mass solvent molecules are essentially pumped away[126]. Subsequent ionization occurs via EI, CI, or fast atom bombardment (FAB). The PB interface offers the advantage of compatible coupling to any mass spectrometer with minor (if any) modification. Such compatibility means that the PB interface enables the coupling of a wide range of LC separations to conventional CI or EI MS procedures.

Offsetting the advantage of accommodating EI for structural information are the disadvantages of low sensitivity, non-linear responses due to matrix effects, and possible discrimination of low mass, volatile samples in the jet-separating region [124, 130, 132]. Although the PB interface has been sparsely used for the confirmation of ivermectin in bovine milk and liver[133] and human metabolites of nicotine in serum and serum[134], its 
susceptibility to matrix effects significantly limits the useful linear dynamic range of the technique[131].

\subsubsection{Fast Atom Bombardment Interface (FAB)}

In a continuous flow or dynamic fast-atom bombardment (CF-FAB) interface, a small liquid stream $(5-15 \mu \mathrm{L} / \mathrm{min})$ is mixed with a FAB matrix solvent such as glycerol, sulfolane, diethanolamine, or triethanolamine and introduced into the low-pressure ion source of the mass spectrometer through an open-ended or frit-terminated (frit-FAB) capillary[124]. Ions are generated by bombardment of the resulting liquid film with accelerated atoms of an inert gas

such as argon or neon. Generated ions are extracted by an electrode and subsequently transferred to the mass analyzer.

FAB is a relatively soft ionization technique primarily producing intact molecular ions. Dynamic FAB alleviates many of the problems associated with static FAB such as differing responses for hydrophobic and hydrophilic analytes and low detection limits due to background signal from matrix related ions[126]. Secondary ions mass spectrometry (SIMS) experiments can also be performed using the same type of interface when using ions rather than atoms for bombardment ionization[124]. Although easy to implement, FAB interfaces are finding limited use in modern literature, primarily due to the introduction of atmospheric pressure ionization (API) techniques.

\subsubsection{Atmospheric Pressure Ionization (API) Techniques}

Atmospheric pressure ionization techniques have quickly become the most widely employed LC/MS interfaces for the analysis of a variety of analytes in forensic and clinical toxicology since their introduction in the mid 1980s. The two most common API techniques 
include atmospheric pressure chemical ionization (APCI) and electrospray ionization (ESI). API interfaces consist of five principle components (Figure 2): (1) the liquid introduction device or spray probe, (2) the atmospheric-pressure ion source region, where ions are generated by means of electrospray ionization (ESI), or atmospheric-pressure chemical ionization (APCI), (3) an ion sampling aperture, (4) an atmospheric-pressure to vacuum interface, and (5) an ion optical system which is responsible for transferring the generated ions into the mass analyzer[135, 136].

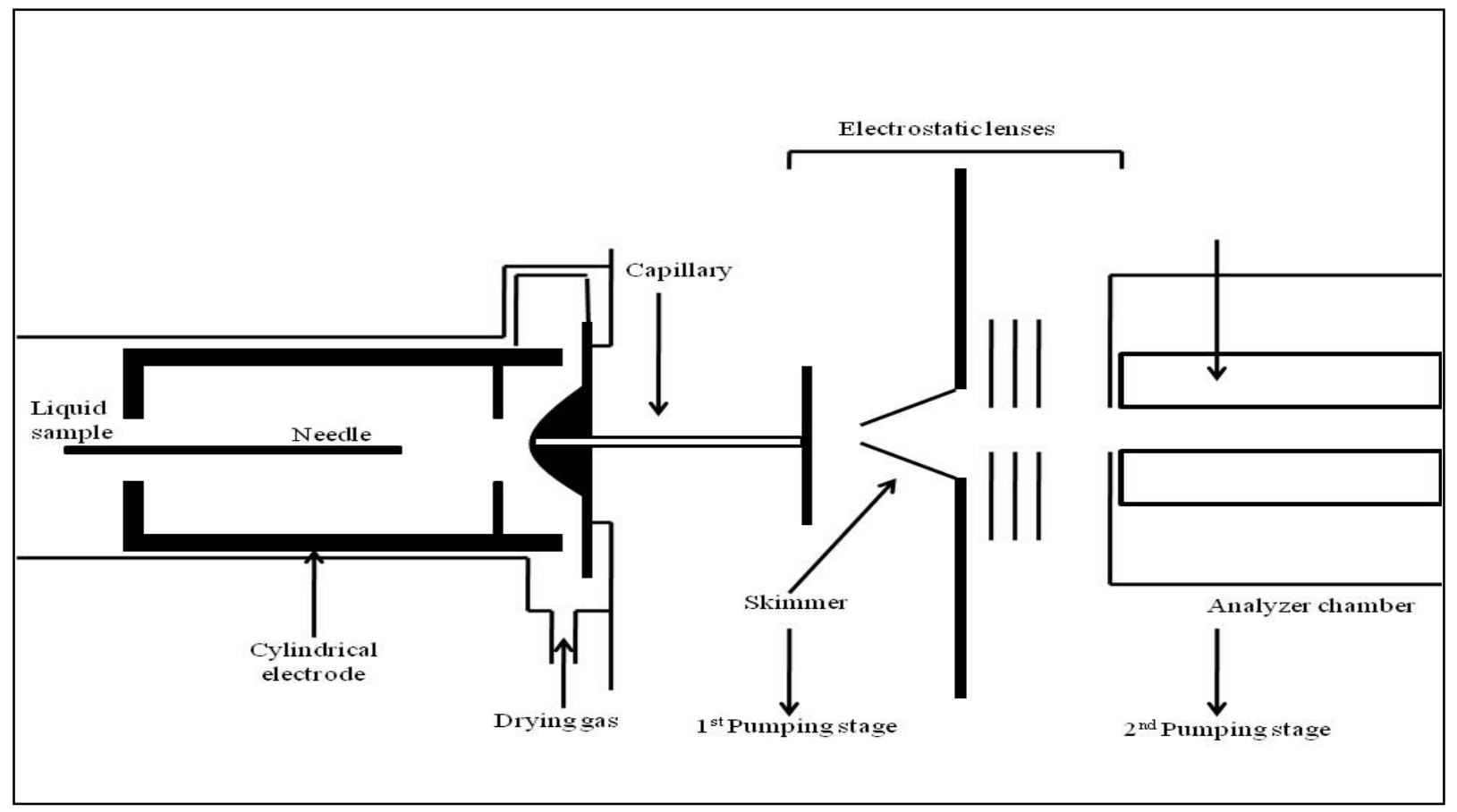

Figure 2: Schematic of an electrospray interface (ESI) (Adapted from Slobodnik 1995).

Specialized design of the last two components is crucial as they determine whether or not the analyst achieves the high ionization efficiencies that are possible with such techniques. Ion losses during transfer from the atmospheric ionization region to the high vacuum region of the mass spectrometer are inevitable, however, proper design of the vacuum interface and ion optics will act to minimize these losses and ensure optimal sensitivity[135]. 
Both ESI and APCI operate by first generating a fine, continuous spray from the liquid chromatographic effluent. Nebulization into the atmospheric-pressure ion source region is achieved pneumatically (APCI), by the action potential of strong electrical field (ESI), or by a combination of the two (pneumatically assisted ESI)[135]. Following generation of the aerosol, gas phase ions are formed by one of several mechanisms explained in more detail below (see sections 2.7.5 and 2.7.6), and these ions, along with solvent vapor and nitrogen bath gas are sampled into a first pumping stage by an ion sampling device. As the mixture of analyte ions, solvent vapors and gases supersonically expand into this first low pressure region, the ions of interest along with other high molecular mass molecules populate the core of this expansion. By employing an appropriate skimmer to sample these core components, analyte ions are transferred into a second pumping stage which is equipped with an ion focusing device and transfer optics which subsequently transfer the ions of interest to the mass analyzer region of the mass spectrometer (pressure $<10^{-3} \mathrm{~Pa}$ )[136].

API techniques such as APCI and ESI offer several advantages over the earlier LC/MS interfaces, four of which were noted by Voyksner as being: (1) Techniques such as APCI and ESI can handle liquid flow-rates that are typically used in LC because the sampling orifice actually acts as the restrictor between the atmospheric-pressure region and the first pumping stage, (2) API techniques are suited to the analysis of highly polar, non-volatile, and thermally labile species typically separated in the liquid phase, (3) API-MS techniques exhibit high sensitivities due to the soft nature and high efficiency of the ionization mechanisms, (4) API-MS techniques are comparatively robust and relative easy to use[137]. LC/MS interfaces relying on the formation of ions at atmospheric-pressure also prevents the problem of vaporized solvent entering the vacuum system of the mass spectrometer[123]. 
APCI and ESI are both soft ionization techniques giving rise to ions which possess minimal internal energies and thus undergo little fragmentation[138]. Ionization of analytes using either APCI or ESI results in the formation of primarily singly-charged ions ("quasi molecular ions") through the addition or subtraction of a proton giving rise to $[\mathrm{M}+\mathrm{H}]^{+}$and $[\mathrm{M}-$ $\mathrm{H}]^{-}$ions respectively[139]. While the formation of singly charged molecular species using API techniques provides little structural information in comparison to hard ionization techniques such as EI, the ease of identification based on, or information pertaining to, the molecular weight of the analyte is maximized.

\subsubsection{Atmospheric Pressure Chemical Ionization (APCI)}

APCI describes the chemical ionization of compounds in an ion source operated at atmospheric-pressure conditions[140, 141]. A typical APCI source requires the following components for successful operation:

- A capillary out of which the chromatographic effluent is sprayed by means of a concentric nebulizer gas.

- A heated vaporizer tube which desolvates the analyte molecules.

- A corona discharge needle which ionizes the molecular mist exiting the vaporizer tube.

- Ion focusing and entrance optics for ion transfer into an area of intermediate vacuum, followed by further focusing elements and skimmers which transport the ions into the high vacuum mass analyzer[142].

Nebulization of the LC effluent into the atmospheric-pressure ion source is performed by a heated nebulizer with pneumatic assistance. Nebulizer temperatures of up to $500^{\circ} \mathrm{C}$ induce solvent evaporation and ions are generated through subsequent gas-phase ion-molecule reactions. 
Reagent ions responsible for the ionization of analyte species are normally created by means of a corona discharge and the subsequent ionization mechanisms are the same as those encountered in traditional medium-pressure chemical ionization $(\mathrm{CI})[126,135]$. Positive ion formation takes place following proton transfer, adduct formation or charge exchange reactions while negative ion formation is achieved by proton abstraction, anion attachment, or electron-capture reactions[126].

APCI is widely employed for the analysis of low to moderately polar compounds. Unlike ESI, solvent evaporation in APCI experiments occurs prior to analyte ionization and the two processes are separate. Solvent evaporation during APCI experiments is supported by the application of high temperatures to the nebulizer while a discharge electrode subsequently acts to ionize solvent molecules, which, after several ion molecule interactions, transfer a charge to the analyte molecule resulting in ionization[124, 139]. APCI is slightly harder than ESI and can induce some degree fragmentation of the molecular species due primarily to the high temperatures employed for the purpose of solvent evaporation[139]. APCI allows for the sensitive determination of analytes possessing moderate polarity and molecular mass (Figure 3) and exhibits high sensitivity for large lipophilic molecules which are not amendable to ionization by other techniques[124, 142]. 


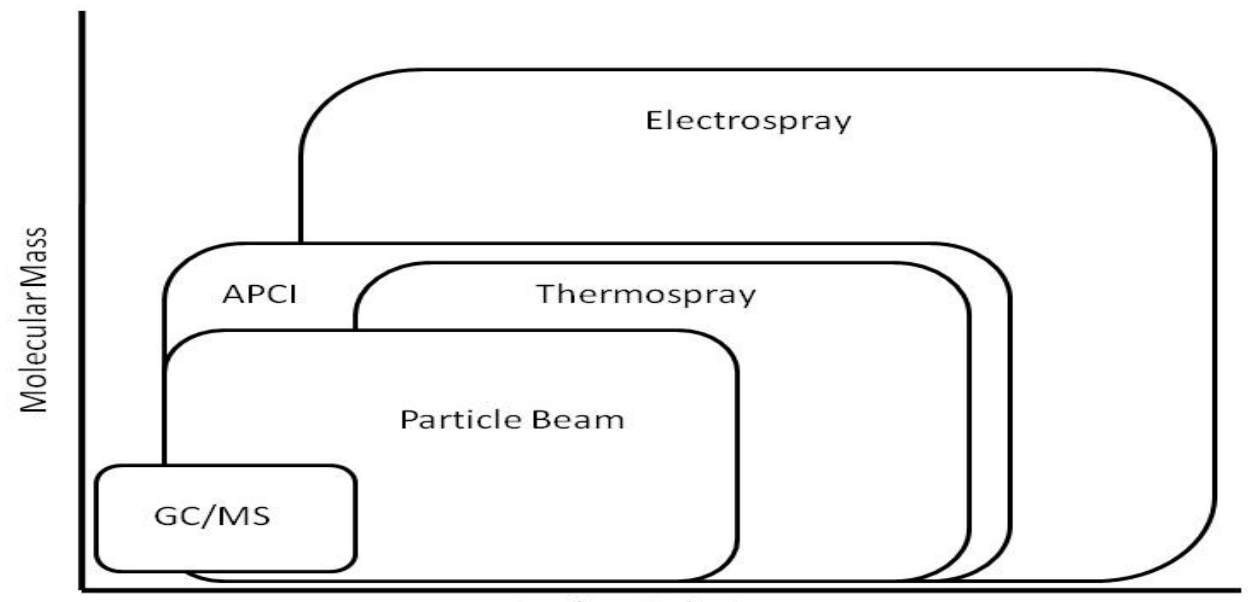

Analyte Polarity

Figure 3: Relationship between molecular mass and analyte polarity for analytes suited to GC/MS and different LC/MS interfaces (Adapted from Maurer 1998).

\subsubsection{Electrospray Ionization (ESI)}

Electrospray ionization (ESI) has quickly become one of the most important and widely employed ionization techniques for the on-line coupling of liquid phase separation methodologies with mass spectrometry (MS). Such coupling has paved the way for numerous electrospray ionization mass spectrometric (ESI-MS) methodologies which have revolutionized the capabilities of clinical and forensic laboratories to detect low levels of analytes in complex biological matrices. Coupling of electrospray ionization with modern mass analyzers provides the analyst with a sensitive, robust and reliable tool for the analysis of femto-mole quantities of non-volatile and thermally labile biomolecules which are not amendable to analysis by traditional techniques[116]. 
The electrospray ionization process employs electrical energy to assist the transfer of ionic species from solution into the gas phase before they are subjected to mass spectrometric analysis (Figure 4). The process of transferring ionic species from a solution into gas phase ions can be described by a three step process:

1. Nebulization of a sample solution into electrically charged droplets (Figure 4).

2. Solvent evaporation (Figure 4).

3. Ion ejection from the highly charged droplets (Figure 4)[116].

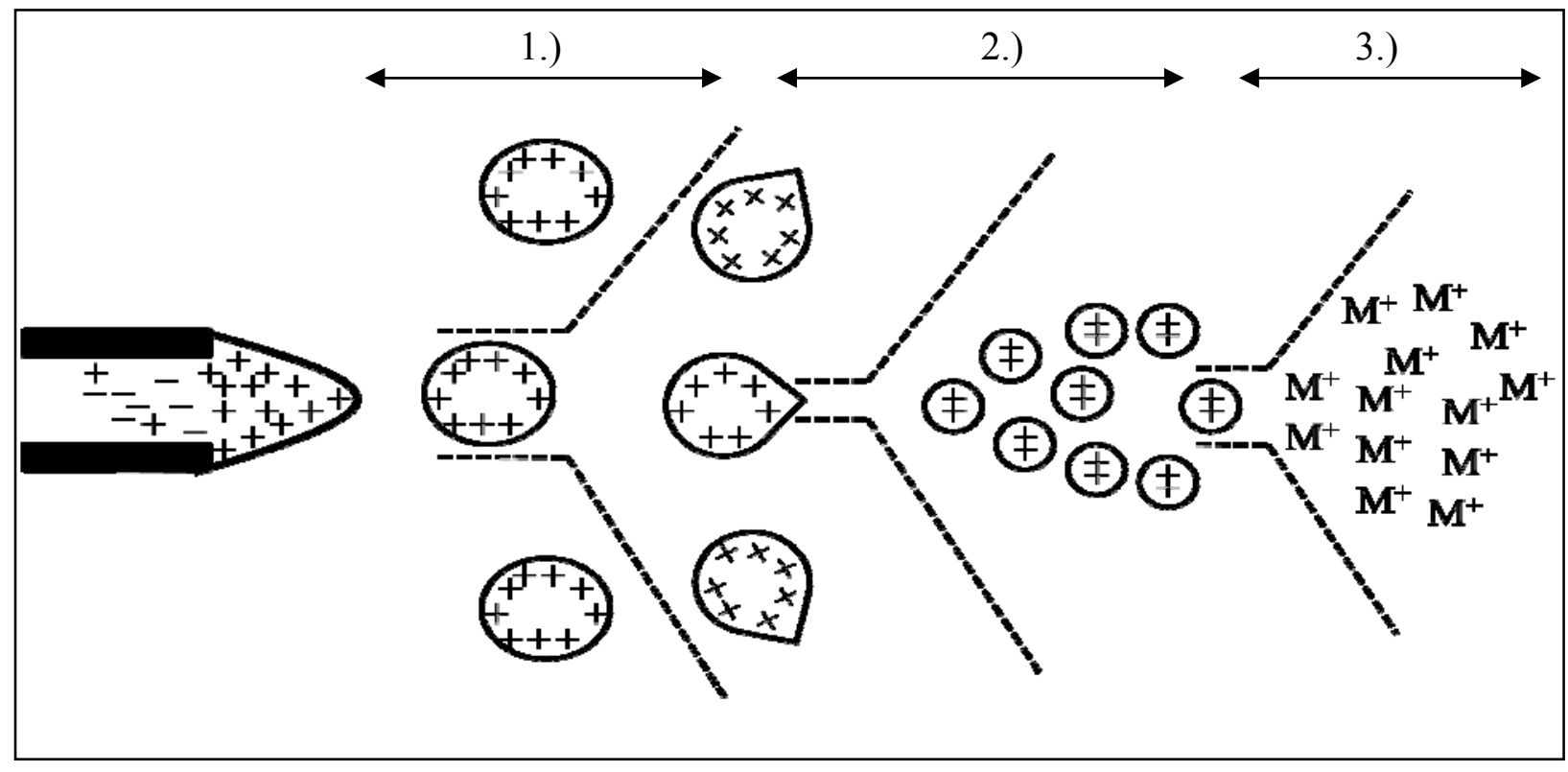

Figure 4: Schematic of electrospray ionization (ESI) showing the three steps of ionization: 1.) Nebulization of a sample solution into electrically charged droplets, 2.) Solvent evaporation, and 3.) Ion ejection from the highly charged droplets (Adapted from Lam 2003).

The initial formation and dispersion of a fine mist of charged droplets is achieved through electrospray nebulization. When a liquid is exposed to a high electrical field, a mist of highly charged droplets is formed in a process termed electrospray nebulization. Electrospray nebulization is achieved by pumping a continuous sample solution through a stainless steel or 
quartz silica capillary tube at a very low flow rate $(0.1-10 \mu \mathrm{L} / \mathrm{min})$. A high voltage $(2-5 \mathrm{kV})$ is applied to the capillary relative to the wall of the surrounding chamber[116]. The high electrical field at the tip of the capillary tube acts to pull like charge toward the liquid front resulting in charge deposition on the surface of the emerging liquid. As charge accumulates at the liquid front, electrostatic repulsions at the exit of the electrospray tip also increase. When the electrostatic repulsion becomes stronger than the surface tension, a highly charged droplet with the same polarity as the capillary voltage is generated $[116,143]$. The point at which droplets emerge from the liquid solution is referred to as the Rayleigh limit and is defined as the point at which coulombic repulsion of the surface charge is equal to the surface tension of the solution[144].

Electrically charged droplets generated at the tip of the capillary tube through electrospray nebulization then pass through the surrounding gas, down a potential gradient, to the counter-electrode. Positively charged droplets are generated when the capillary is held at a more positive potential than the counter-electrode causing positive charge to accumulate at the capillary tip. A reversal of the electric field will result in the formation of negatively charged droplets travelling down a potential gradient to a counter-electrode being held at a more positive potential. The formation of micrometer-sized droplets depends on the liquid's flow rate, surface tension and electrolyte concentration[143]. A significant increase in any one of the three aforementioned variables can greatly decrease the ability of the electric field to generate the desired aerosol required for ion transfer to the mass spectrometer. Small increases in a liquid's flow rate, surface tension or electrolyte concentration may be overcome by increasing the electrical field at the tip of the capillary however care must be taken to ensure that the increase in electric field energy does not give rise to an electrical discharge[143]. 
The second step in the process of transferring condensed phase ion into gas phase ions suitable for mass spectrometric analysis involves solvent evaporation. Electrospray nebulization produces electrically charged droplets when charge repulsion exceeds the cohesive force that holds the liquid together. Electrically charged droplets then traverse down a potential gradient toward the entrance to the mass spectrometer, and generate analyte ions by one of several proposed mechanisms.

The coulomb fission mechanism proposes that the increased charge density due to solvent evaporation causes large droplets to undergo size reduction into successively smaller droplets which eventually consist of a single analyte ion[145]. Solvent evaporation is aided by elevated source temperatures and/or the introduction of a stream of nitrogen drying gas. The reduction in droplet size through solvent evaporation results in an increase in charge density at the surface of the droplet. Further deformation of the droplet occurs as a result of the shear forces experienced by the droplet during its flight through a dense desolvation gas toward the counterelectrode[143]. As the droplets evaporate, the increasing coulomb forces exceed the surface tension causing the droplet to undergo fission (Figure 4). Successive fissions ultimately give rise to droplets containing a single analyte molecule possessing and retaining the droplet charge as the remaining solvent evaporates[146]. A second mechanism for the formation of single analyte ions assumes that the increase in charge density resulting from solvent evaporation eventually results in coulombic repulsions that exceed the liquid's surface tension and as a result, ions are released from the droplet surfaces[147]. This mechanism is termed ion evaporation and theorizes that microdroplets with an approximate radius of $10 \mathrm{~nm}$ resulting from successive fissions, do not experience further disintegration to smaller droplets but rather emit ions from their surface in order to alleviate charge repulsion[116, 143]. 
While ionization of analytes during ESI experiments occurs predominantly through condensed phase equilibrium or charge transfer complexation in solution, gas phase interactions, occurring after the analyte has been released from solution also exist[148]. Gas phase interactions occur following droplet emission and prior to the analyte reaching the mass analyzer. As a result, such reactions can have a significant effect on MS response[149]. Gas phase interactions which result in analyte charging during ESI experiments occur through gas-phase proton-transfer reactions. Such a phenomenon exists primarily because the gas phase basicity, and therefore proton affinity, of certain molecules can be markedly different to their basicity in solution. Initial charging of molecules during ESI experiments occurs in the condensed phase through equilibrium and charge transfer according to the molecules solution-phase basicity. Following electrospray nebulization into the gas phase, molecules that were protonated in solution can yield their protons to solvents or analytes with greater gas-phase basicity[149]. Such gas-phase proton transfer reactions take place when there is an inversion in the order of basicity among a series of molecules proceeding from solution into the gas phase[150]. Analyte molecules which are sprayed and ejected as neutrals can become positively charged through gasphase interactions with solvent ions which exhibit a higher basicity in solution but possess inferior gas-phase basicity. As a result of this basicity inversion, the protonated solvent molecule yields its proton to the more basic gas-phase analyte resulting in gas-phase charging. Increased signal through gas-phase analyte charging can greatly increase sensitivity in ESI-MS experiments however analyte response may also be completely suppressed in situations where the electrospray solvent has a higher gas-phase proton affinity than the analyte[151]. It is therefore imperative to select an electrospray solvent which possesses a lower gas-phase proton affinity than the analyte of interest. 
Of the above interface technologies, most research and further development efforts are focused around the atmospheric pressure ionization (API) techniques, specifically, atmospheric pressure chemical ionization (APCI) and electrospray ionization (ESI).

\subsection{LC/MS/MS and its Applications in Forensic Toxicology}

The vast majority of LC/MS instrumentation employs APCI or ESI due to the large number of compounds which are amendable to soft ionization techniques, regardless of their polarity or molecular weight (Figure 3). API techniques produce characteristic ions depending on the ion mode in which the spectra are obtained. In positive-ion mode the base peak is normally the protonated molecular ion $[\mathrm{M}+\mathrm{H}]^{+}$which is often accompanied by less abundant metal adducts such as $[\mathrm{M}+\mathrm{Na}]^{+}$and $[\mathrm{M}+\mathrm{K}]^{+}[152,153]$. When spectra is obtained in negative-ion mode, the deprotonated molecular species $[\mathrm{M}-\mathrm{H}]^{-}$generally constitutes the base peak[152]. While soft ionization techniques such as APCI and ESI provide abundant ions of the molecular species, leading to increases in sensitivity, they yield very few, if any, fragments and therefore

provide relatively little structural information and limited selectivity[154]. To order to increase the selectivity of experiments employing soft ionization techniques, tandem mass spectrometry (MS/MS) is applied to induce fragmentation of the molecular species and give rise to structurally significant product ions[155].

Tandem mass spectrometry employs two stages of mass analysis to examine the fragmentation of analyte ions within a mixture of ions. The increased selectivity afforded by tandem mass spectrometry has been widely utilized in the fields of forensic and clinical toxicology where analyte identification can prove challenging due to the complex nature of biological matrices[154]. Tandem mass spectrometers provide an added dimension of mass spectral information by creating ionic species from a sample, mass selecting a precursor ion, 
inducing fragmentation of that selected ion and obtaining the mass spectrum of the structurally significant fragments[156]. The goal of tandem mass spectrometry is to provide the analyst with additional structural information not normally yielded by soft ionization techniques, while simultaneously retaining the sensitivity afforded by the soft ionization. By employing two or more mass spectrometers with a collision cell positioned in between, tandem mass spectrometry ensures increases in selectivity by providing characteristic molecular fragments generated by collision-induced dissociation (CID)[157]. Tandem mass spectrometers allow for the selective determination of multiple analytes within a single run by monitoring multiple ions or fragmentation transitions characteristic to individual analytes. In addition, the baseline separation of analytes from matrix interferences, which is a must for HPLC employing UV or MS detection, is not required in MS/MS experiments because of its inherent selectivity[157].

Tandem mass spectrometry is achieved using a variety of instruments which can be classified into two categories of tandem mass spectrometers. The first category is made up of instruments in which two mass spectrometers are assembled in tandem such tandem massanalyzing quadrupoles, tandem time-of-flight analyzers or a combination of the two (QTOF). The coupling of several different analyzers for the purpose of MS/MS experiments have been described in the literature. The sequential combination of mass analyzers is generally intersected by a collision cell which is designed to induce dissociation of the precursor ions through collisions with an inert gas such as argon. Product ions resulting for CID are then analyzed in the second quadrupole. Experiments performed using the sequential combinations of mass analyzers are referred to as tandem-in-space mass spectrometry. Tandem mass spectrometry experiments can also be performed using another category of instruments which are characterized by their ability to store ions. Mass analyzers which are capable of storing ions for subsequent analysis 
include the ion cyclotron resonance (ICR) and the quadrupole ion trap mass[154]. Mass analysis is performed by creating precursor ions and storing them in a radio frequency (rf) trapping field. Following ion production, separation and storage, precursor ions can be excited and caused to fragment during a selected time period by the application of additional rf waveforms. Fragmentation of the precursor ions leads to the generation of characteristic product ions which can be observed in a mass spectrum by utilizing the highly efficient ion manipulation capabilities of such analyzers $[154,158]$. This process may be repeated to obtain the mass spectrum of fragments over several generations without the need to transfer ions from one vacuum chamber to the next, rather, a single chamber utilizing a quadrupole rf field is used for all analyses[154, 158]. Experiments performed using mass analyzers capable of ion storage are referred to as tandem-in-time mass spectrometry. Tandem mass spectrometry combined with the appropriate chemical separation technique such as liquid chromatography or gas chromatography provide analysts with impressive selectivity and sensitivity by eliminating a large majority of chemical noise, resulting in superior selectivity and limits of detection relative to single mass spectrometry experiments[158]. The majority of tandem mass spectrometric experiments performed in recent times have employed a "tandem-in-space" approach and the most widely used tandem mass spectrometer of this category is the triple quadrupole. 


\subsection{Triple Quadrupole Mass Spectrometer}

\subsubsection{Introduction}

The triple quadrupole assembly was first introduced by Yost and Enke in 1978 for the purpose of mixture analysis and structural elucidation[156]. A quadrupole is a mass analyzer consisting of four co-linear rods, set parallel to each other (Figure 5).

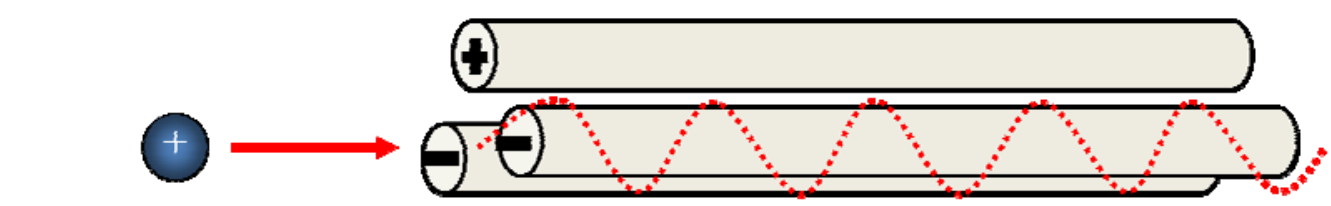

From ion source

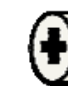

To detector

Figure 5: Schematic of a quadrupole mass analyzer.

Each opposing pair of rods is connected electrically and an RF voltage is applied between the two rod pairs. A DC voltage is then superimposed onto the RF voltage and it is the ratio of these two voltages that enables the quadrupole to perform mass analysis. Depending on the exact potential applied to the rods, only ions with a certain $\mathrm{m} / \mathrm{z}$ ratio will pass through the quadrupole and reach the detector because the applied voltages affect the trajectories of ions travelling down the flight path which is centered between the two sets of rods. Ions with unstable trajectories in the oscillating electric fields will collide with the rods and exit the system through vacuum. For a given voltage, only ions with a certain $\mathrm{m} / \mathrm{z}$ ratio will pass through the quadrupole and reach the detector while all other ions are essentially filtered out. Quadrupole mass analyzers were employed in the first desktop LC/MS systems and are still the most commonly employed and least expensive mass analyzers used in LC/MS instruments[159]. 
The stability and motion of ions in a quadrupole is significantly different from those observed in field-free regions and in magnetic and electrostatic sectors[160]. Ion trajectories in dynamic instruments such as quadrupole devices are influenced by time-dependant forces which complicate their mathematical prediction relative to sector instruments. The motion of ions in a quadrupole field can be mathematically described by solutions to the second-order linear differential Mathieu equation (Equation 10).

$\left(\left(d^{2} u\right) /\left(d \zeta^{2}\right)\right)+\left(a_{u}-2 q_{u} \cos 2 \zeta\right) u=0$

Equation 10

Mathieu was able to describe solutions in terms of regions of stability and regions of instability while investigating the mathematics of vibrating stretched animal skins[160]. The Mathieu equation can be applied to describe the regions of stability and instability during ion trajectory in a quadrupole device. The Mathieu mass stability diagram shown in Figure 6 is derived from the Mathieu equation and illustrates regions of stable and unstable trajectory for two ion masses (A and B) entering a quadrupole at the same time. 


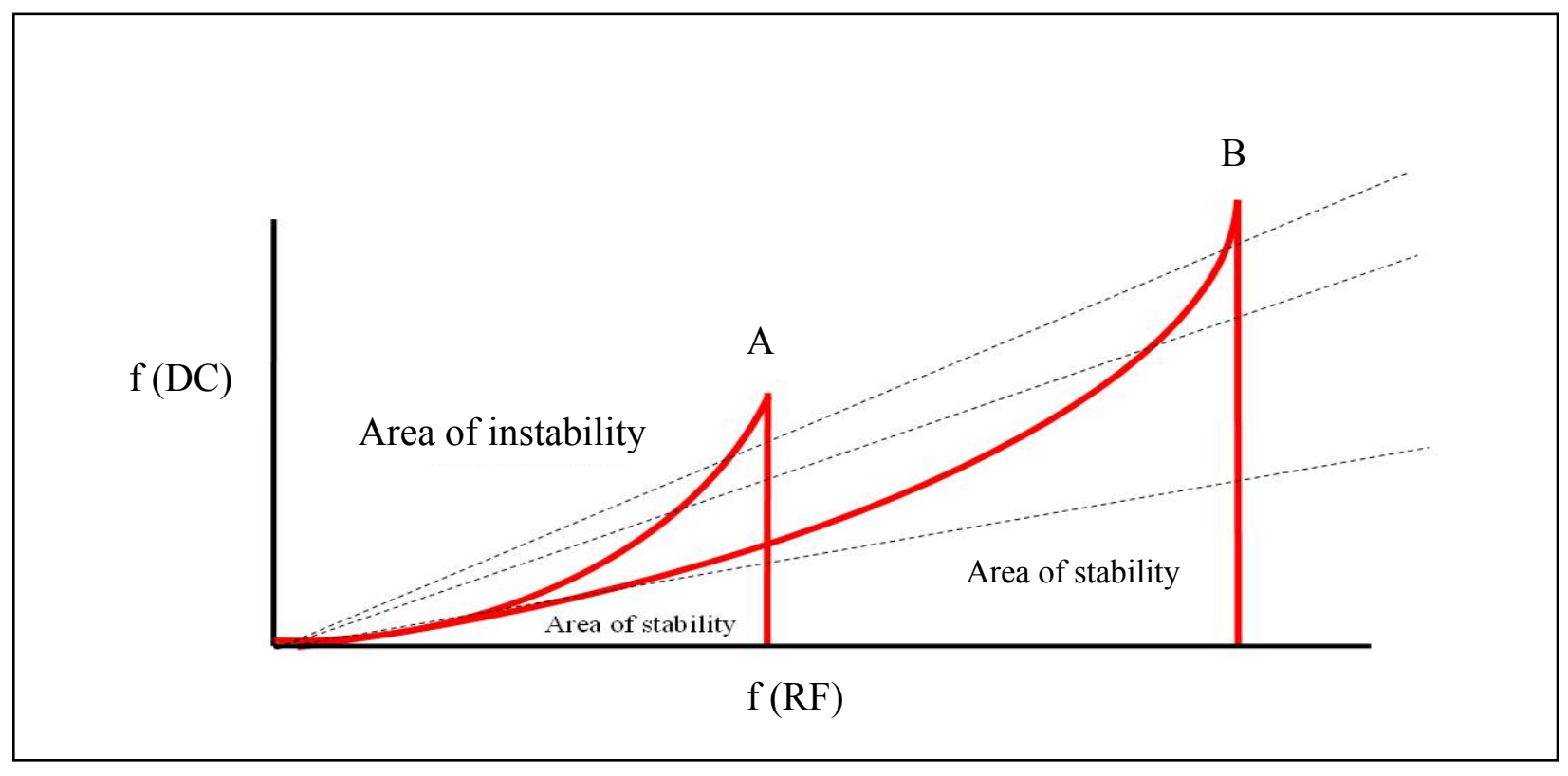

Figure 6: Mathieu stability diagram of two masses (A and B) in a quadrupole mass filter. Voltage coordinates corresponding to points under the stability curve represent stable trajectories while all other voltage conditions result in unstable ion trajectory in the quadrupole device.

Any of the RF/DC conditions under the stability curve represent conditions of stable trajectory during which that mass will pass through the quadrupole to the detector. RF/DC conditions appearing only under the left hand curve will allow only mass A to pass through the quadrupole while mass B will experience unstable trajectory and be removed from the quadrupole through vacuum. Similarly, any of the RF/DC condition appearing only under the right hand curve will only allow mass B to pass through the quadrupole. Figure 6 shows three dotted lines which represent typical quadrupole scan lines which pass through the stable regions of each curve under different RF/DC conditions. If the slope of the RF/DC scan is steep, similar to the top line in Figure 6 the spectral peaks will be narrow and well separated due to the smaller regions of stability encountered by those scan conditions. If the slope of the RF/DC scan is shallow, as represented by the middle line in Figure 6, spectral peaks will be wider and poorly 
separated leading to less resolving power. If the slope of the RF/DC scan is too shallow, as represented by the bottom line in Figure 6, masses A and B will pass through the quadrupole without being separated due to significant overlap in regions of stability[161].

Understanding the principles of the Mathieu equation and corresponding stability plots enables the analyst to monitor the presence and abundance of a particular mass or scan the mass range by varying the $\mathrm{rf}$ and dc voltages applied across the rods and obtaining the corresponding mass spectrum. Only the first (Q1) and third (Q3) quadrupoles are operated using a combination of $\mathrm{rf}$ and dc voltages necessary for mass analysis, while the second quadrupole (Q2) is operated with a fixed rf voltage only and therefore allows ions of all masses to pass through it. Q1 and Q3 both have the ability to perform mass analysis while Q2 is employed as a collision cell with ion focusing properties in which precursor ions, having already passed through Q1, undergo collision induced dissociation (CID) into characteristic product ions which can then be scanned or transmitted using Q3 (Figure 7)[154]. Collision induced dissociation is achieved by selecting a parent mass to pass through Q1 and into the quadrupole collision cell where it collides with atoms of an inert gas, usually argon, and acquires energy which leads to its subsequent decomposition into characteristic product or daughter ions[118]. CID is widely employed to obtain structurally characteristic fragmentation patterns which are used for the unequivocal identification of analytes in complex matrices[162]. The reproducibility of CID is dependent upon the chosen collision gas, collision energies, and collision gas flow. While specific parameters may be optimal and constant on one instrument, significant variation is common between instruments meaning that CID product ions may also be instrument and laboratory dependant. Variations in CID parameters, and therefore product ions, between laboratories 
complicate the development of reference spectral libraries. For this reason LC-MS/MS does not currently possess the same discriminative power as GC/MS employing EI.
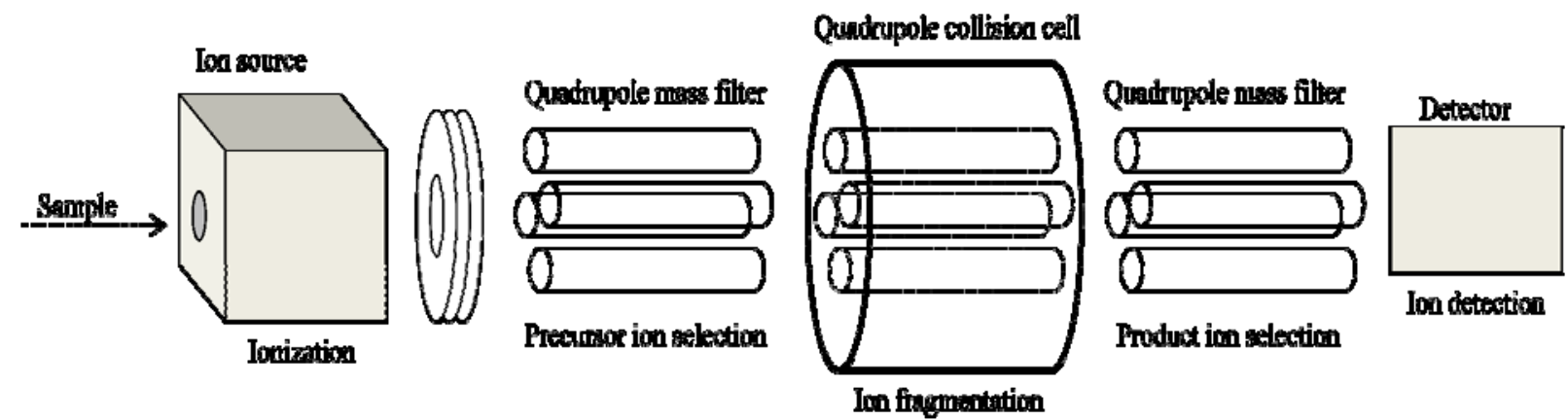

Figure 7: Schematic representation of a triple quadrupole mass spectrometer.

\subsubsection{MS Operating Modes}

Mass analysis can be performed utilizing one or both of the mass analyzers when employing triple quadrupole instruments as they have the ability to operate in the single mass spectrometry (MS) mode or tandem mass spectrometry (MS/MS) mode. Single mass spectrometry experiments are performed using one of three main MS scan modes (Table 3). The most widely employed MS mode is the MS1 mode in which the first quadrupole (MS1) is employed as a mass filter while the collision cell and MS2 allow all masses exiting MS1 to pass through to the detector. MS1 mode is the most sensitive method for performing MS analysis and it is directly analogous to experiments employing a single-quadrupole mass spectrometer for the purposes of scanning a mass range[163]. The MS2 mode of operation is also employed for MS experiments however it provides optimum performance with scan speeds greater than 2000 $\mathrm{Da} / \mathrm{s}[163]$. MS2 is achieved by allowing ions of all masses to pass through MS1 and Q2 while employing the fast MS scanning capabilities of MS2. The SIR (selected ion recording) mode of operation is employed as a quantitative tool for MS analysis when no suitable or reproducible fragmentation pattern of the parent molecule can be identified to allow for a more selective 
MS/MS analysis[163]. SIR (or SIM) is directly analogous to single quadrupole mass

spectrometry experiments in which a single ion of interest is pre-selected to oscillate through the quadrupole.

Table 3: MS operating modes using the triple quadrupole mass spectrometer.

\begin{tabular}{|c|c|c|c|}
\hline Operating mode & MS1 & Collision cell & \multicolumn{1}{c|}{ MS2 } \\
\hline MS1 & Resolving (scanning) & \multicolumn{2}{c|}{ Pass all masses } \\
\hline MS2 & \multicolumn{2}{|c|}{ Pass all masses } & Resolving (scanning) \\
\hline SIR & Resolving (static) & \multicolumn{2}{r|}{ Pass all masses } \\
\hline
\end{tabular}

\subsubsection{MS/MS Operating Modes}

By operating in tandem mass spectrometry (MS/MS) mode by utilizing the mass analysis capabilities of Q1 and Q3, an additional dimension of mass spectral information is provided. The combination of two mass analyzers provides the analyst with the option to acquire information using additional operating modes not amendable to traditional single-quadrupole MS analysis. Because both analyzers can be operated in the scan or selected-ion mode (SIR), the number of possible combinations and therefore operating modes is extended to four (Table 4)[120].

Table 4: MS/MS operating modes of the triple quadrupole mass spectrometer.

\begin{tabular}{|c|c|c|c|}
\hline Operating mode & MS1 & Collision cell & MS2 \\
\hline Product & Static & Pass all masses & Scanning \\
\hline Precursor & Scanning & Pass all masses & Static \\
\hline MRM & Static & Pass all masses & Static \\
\hline Constant neutral loss & Scanning & Pass all masses & Scanning \\
\hline
\end{tabular}

When operating in product (daughter) ion scan mode, the first quadrupole is static and allows only ions with a specific $\mathrm{m} / \mathrm{z}$ (precursor ions) to pass through into the collision cell. The collision 
cell operates as normal by inducing fragmentation of the precursor ions through energetic collisions with atoms of an inert gas such as argon. Fragmentation produces characteristic product ions which are then scanned by the third quadrupole which is set to scan over an appropriate mass range. A product ion spectrum (formerly referred to as a daughter ion spectrum) is obtained when operating in product ion scanning mode. (Figure 8)[163]. Product ion scanning is most commonly employed in method development to identify fragmentation patterns of a specific analyte. Optimization of CID conditions is achieved using product scanning by identifying collision parameters which maximize the yield of specific product ions passing through Q3 and reaching the detector. Product scanning is also employed for structural elucidation as product ions are characteristic of precursor ions. Product ion scanning is widely employed for the unequivocal identification of drugs and their metabolites in complex biological matrices. Chemical separation of the analyte from the complex matrix is achieved by the chromatographic separation and the first quadrupole, while fragmentation of the analyte in the second quadrupole provides structural information[120,163]. A product ion spectrum displays the ions produced by fragmentation of a known parent ion[118].

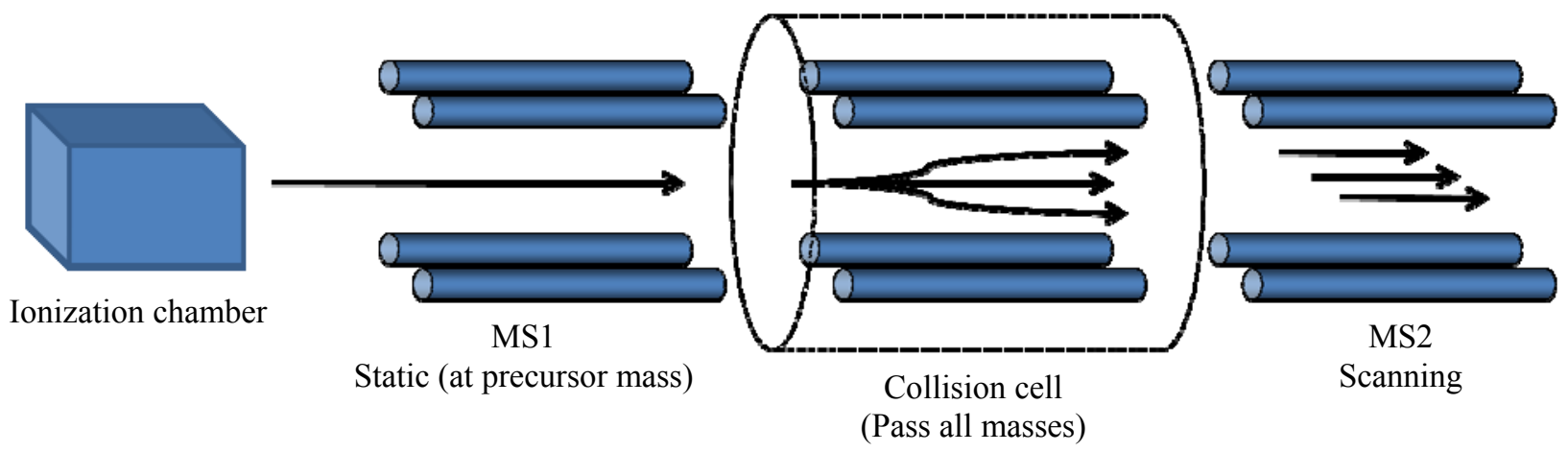

Figure 8: Schematic representation of product (daughter) ion scanning mode. 
Precursor ion scanning is achieved by scanning the first quadrupole over a chosen mass range while the third quadrupole is set to pass only ions with a particular $\mathrm{m} / \mathrm{z}$ (product ions) (Figure 9). Precursor scanning allows all ions to pass through Q1 and undergo CID in the second quadrupole however only desired product ions will be allowed to pass through the third quadrupole. By constantly scanning the first quadrupole, precursor ions which fragment into the desired product ions will be identified. Ions which pass through the first quadrupole will only be detected if they produce the pre-selected product ions through fragmentation in the collision cell[154]. Precursor scanning is typically employed to corroborate information obtained from product scans during structural elucidation experiments. Precursor scanning is also employed to identify possible precursors of a common product ion[163].

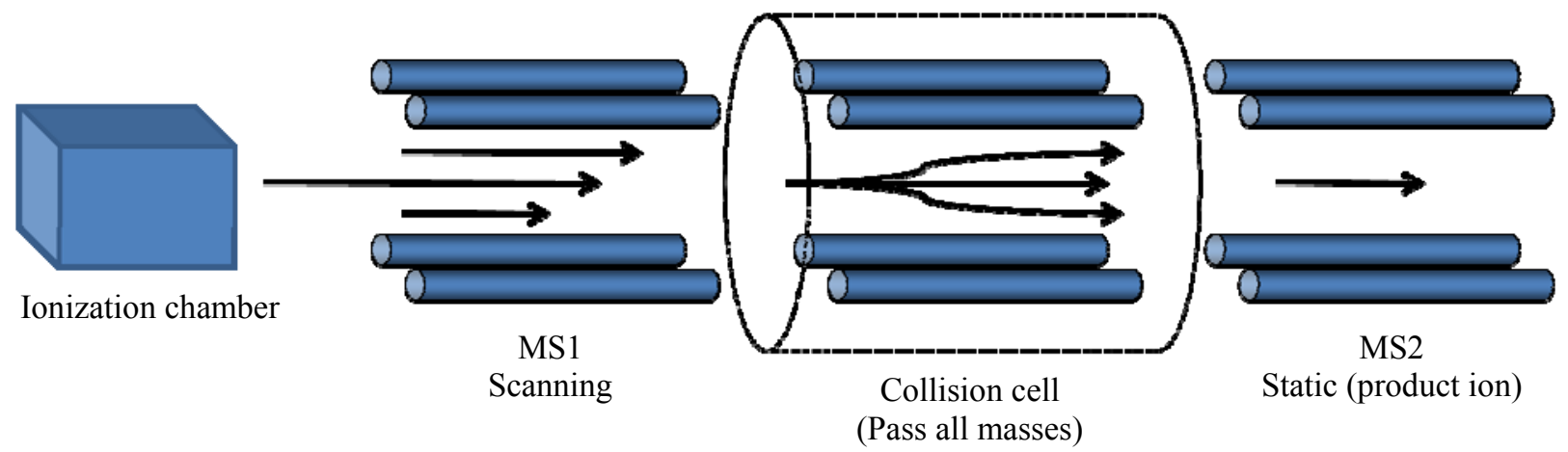

Figure 9: Schematic representation of precursor ion scanning mode.

Multiple reaction, or selected reaction monitoring (MRM or SRM) mode is a highly selective tandem mass spectrometry equivalent of SIR. MRM experiments are performed by setting Q1 static to only transmit ions with specific $\mathrm{m} / \mathrm{z}$ corresponding to the precursor ion of interest. Following fragmentation in the collision cell, only characteristic product ions of the precursor are transmitted through Q3 which is also held static (Figure 10). MRM experiments can only be performed when fragmentation patterns for the analyte have been determined. Once 
characteristic product ions have been identified and CID parameters have been optimized, Q3 can be programmed to allow only product ions with certain $\mathrm{m} / \mathrm{z}$ values through to the detector. MRM mode is widely used for quantitative analysis is MS/MS experiments. Dwell times can be maximized because both Q1 and Q3 are held static to only allow through ions with pre-selected $\mathrm{m} / \mathrm{z}$ values (precursor and product ions respectively), meaning that enhanced sensitivity is achieved compared to single mass spectrometry experiments performed in the scanningmode[163]. MRM is most commonly employed to quantify target analytes in complex matrices such as drugs and /or metabolites in biological samples. Because individual transitions are monitored in both SIR and MRM the product of such scans is not a mass spectrum, rather a chromatogram[163]. While the number of MRM transitions required for an unequivocal identification is still largely debated, most laboratories monitor two transitions and require product ions to be present at a predetermined ratio. Ion ratios are generally determined using quality control standards which are analyzed prior to patient samples. Increasing the number of MRM transitions enhances selectivity at the cost of sensitivity due to decreased dwell times.

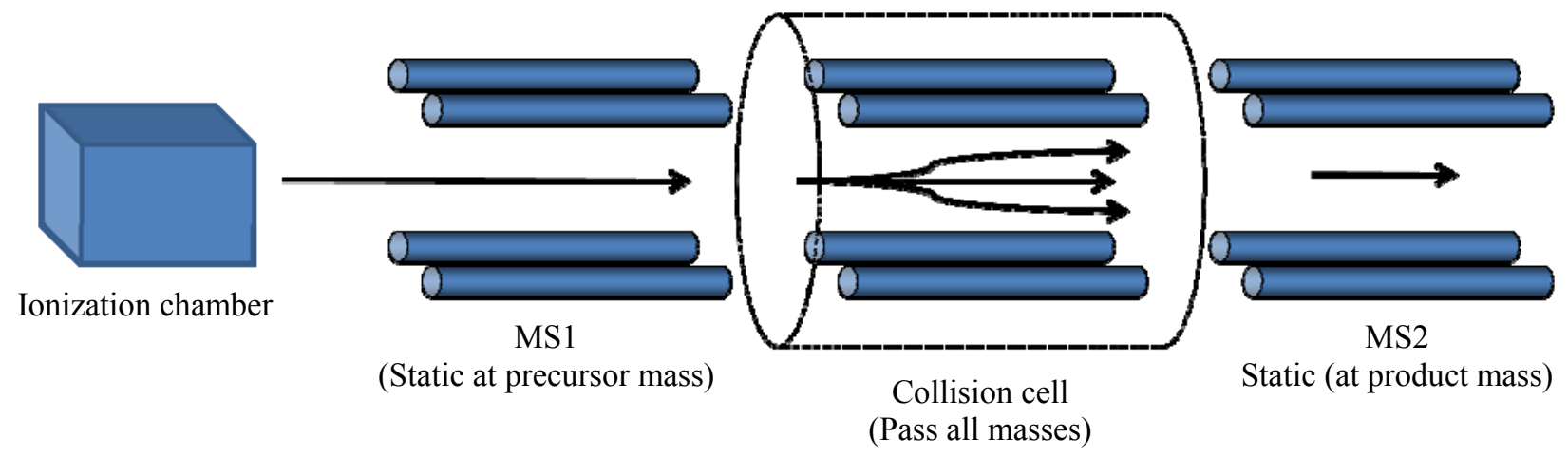

Figure 10: Schematic representation of multiple reaction monitoring (MRM) mode.

Neutral loss tandem mass spectrometry experiments identify the loss of a specific neutral fragment (eg. expulsion of $\mathrm{H}_{2} \mathrm{O}$ ) or functional group from an unspecified precursor (Figure 
11)[163]. Neutral loss scanning requires that both Q1 and Q3 are scanned simultaneously with a constant $\mathrm{m} / \mathrm{z}$ difference between them corresponding to the mass of the lost fragment or functional group[154]. When the scanning of Q1 and Q3 is synchronized, Q3 determines whether precursor ions transmitted through Q1 have lost a fragment corresponding to the mass difference between the two scanning spectrometers. If Q3 detects a fragment loss corresponding to the mass of the neutral fragment of interest, the loss registers at the detector and the mass spectrum displays all precursor ions that experienced a mass loss corresponding to the fragment of interest[164]. Neutral loss scan mode is most commonly employed to screen for a certain class of compounds by identifying all ions which undergo the loss of a given neutral fragment through fragmentation[154, 164]. Screening for a certain class of compounds which are known to follow a characteristic fragmentation pathway, involving the loss of a certain neutral fragment or functional group, could be achieved by employing a neutral loss scan. Neutral loss scanning is commonly employed in early drug discovery for the identification of drug classes. An example is the identification of glycerol phosphatidylcholine phospholipids (GCP) using a constant-neutralloss scan of $\mathrm{m} / \mathrm{z}$ 59. Under certain CID conditions the dominant fragmentation of GCP's is the loss of trimethylamine (m/z 59) from the polar head group. Detection of a neutral loss of mass 59 Da at Q3 would indicate the presence of a GCP and allow for differentiation of this class from other phospholipid classes present in complex mixtures[165]. 


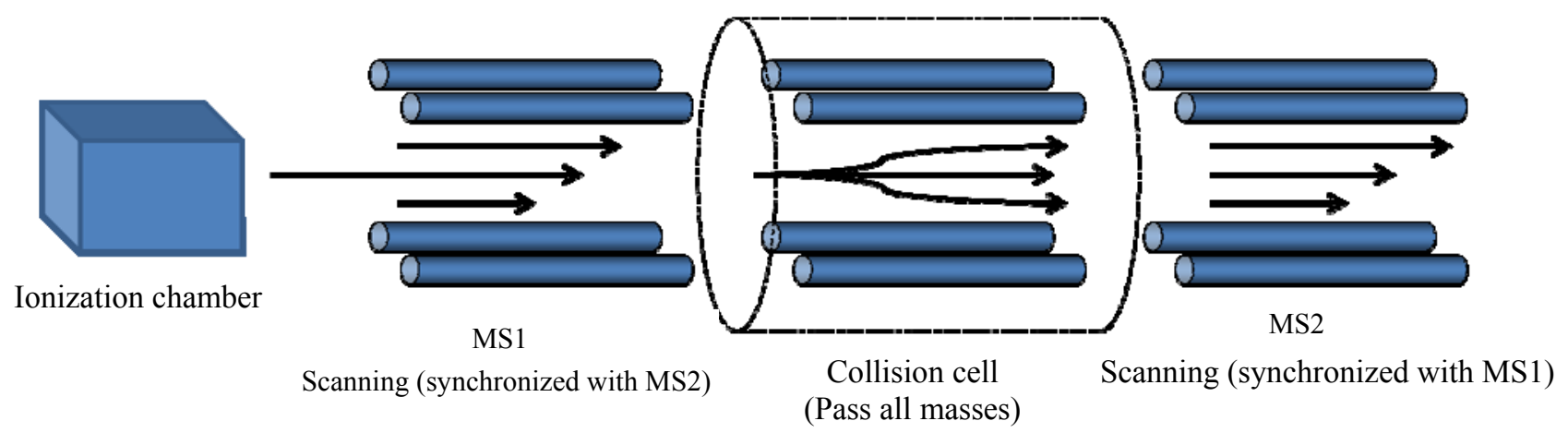

Figure 11: Schematic representation of neutral loss scanning mode.

\subsection{Mass Spectral Criteria for a Positive Identification}

Prior to the introduction of atmospheric pressure ionization techniques such as ESI, and APCI, a large majority of toxicological analyses were performed using GC/MS employing EI. Electron ionization (EI) yields fragment ions which are very characteristic of, and therefore provide significant information pertaining to, molecular structure. This is due primarily to the ability of EI to produce odd electron molecular ions. Dissociation of odd electron molecular ions produces fragment ions which are significantly more characteristic of molecular structure than are even electron molecular ions[117]. The extensive fragmentation of odd electron molecular ions into structurally significant fragment ions under EI conditions provides the analyst with an EI mass spectrum possessing an unusually high content of structural information. This additional structural information afforded by EI mass spectra has allowed for the development of EI reference spectral libraries. Identification of unknown compounds is achieved by 'library matching' which involves matching the EI spectrum of an unknown compound with one or several contained within the library database. Library matches are made by comparing molecular ions, relative abundances of fragment ions, and isotope patterns of the unknown compound with reference spectra contained in the library. EI spectral libraries are readily available and can be 
installed into instrument software to allow for automatic library matching following data acquisition of an unknown compound. For this reason, EI remains the method of choice for the routine, efficient, identification of unknown compounds at trace levels[166].

Identification of unknown compounds using API techniques is achieved using a different approach due to the relatively small amount of structural information afforded by soft ionization. While the protonated molecular ions produced during API experiments assist in molecular weight determinations, little information is provided to assist with structural elucidation. As a result, ESI or APCI spectral libraries are not practical and do not currently exist to aid with unknown identification. Due to the lack of structural information provided by soft ionization, API techniques are not commonly employed for broad based, general unknown screening. General unknown screening in complex biological matrices employing API techniques would prove impractical as identifications would be based solely on the presence or absence of protonated molecular ions. For this reason, GC/MS employing EI remains the most commonly employed technique for broad based drug screening. Identifications are based on full EI spectra containing characteristic information that can be automatically matched with reference spectra.

While API techniques do not naturally lend themselves to general unknown screening applications, several tandem mass spectrometric screening techniques employing soft ionization have been reported in the literature [167-170]. The identification of analytes using APILC/MS/MS is normally achieved using retention times and by monitoring the presence of 2-3 multiple reaction monitoring (MRM) ion channels. Unlike GC/MS screening in which identifications are made based on pre-existing spectral libraries, API-MS/MS methods must be developed through the analysis of standards. A reference standard of each analyte must first be infused into the mass spectrometer to obtain ionization, and CID fragmentation information. 
Following optimization of the mass spectral conditions, standards must be injected onto the LC to determine individual retention times. The screening method is then built by designating each analyte a characteristic retention time and a minimum of one characteristic MRM transition which is monitored during the analysis of unknowns. Screening methods based on monitoring MRM transitions present additional problems as the number of analytes increase. Monitoring the presence of analytes based on MRM transitions requires the mass spectrometer to continuously scan the appropriate mass range. As the number of analytes increase, dwell times and therefore sensitivity decreases. Additional screening selectivity may be achieved by monitoring more than one MRM transitions for each analyte, however, the addition of more MRM transitions to the MS/MS method further limits dwell times and decreases overall method sensitivity. Care must be taken when optimizing dwell times to ensure that sufficient data points are obtained across each chromatographic peak. Many forensic samples contain very low concentrations of analytes and attempts to increase screening sensitivity by increasing dwell times may result in an inability to accurately characterize chromatographic peaks as a result of insufficient data points.

Due to the nature of data acquisition, API-MS/MS techniques are better suited to, and more commonly employed for confirmation analysis. Confirmation methods employing APIMS/MS are generally employed to confirm the presence of, and quantitate a specific drug. These confirmation methods may be designed to detect and quantitate a single analyte or several members of a certain family of drugs, such as the opiates. Specific LC-MS/MS methods are again developed through the analysis of standards and positive identification criteria normally include retention times, and the presence of 2-3 MRM transitions in the correct abundance. During the analysis of reference standards, the most abundant MRM transition for each analyte is routinely employed at the quantifying ion, while the second most abundant transition is 
employed for qualitative identification purposes. Confirmation methods are rarely designed to detect and quantify more than a few drugs at any one time meaning that dwell times, and therefore sensitivity can be maximized. Confirmation methods employing API-MS/MS combine the increased sensitivity afforded by soft ionization with the inherent selectivity of tandem mass spectrometry.

\subsection{Mass Range and Resolution}

The mass range of any mass spectrometer refers to the range of $\mathrm{m} / \mathrm{z}$ values that can be characterized with sufficient resolution to characterize adjacent peaks. Mass resolution refers to the ability of the mass spectrometer to distinguish between two peaks of differing $\mathrm{m} / \mathrm{z}$ values within a single mass spectrum. A numerical expression of resolution can be obtained from the ratio $\mathrm{m} / \Delta \mathrm{m}$, where ' $\mathrm{m}$ ' is the nominal $\mathrm{m} / \mathrm{z}$ value of one of the compounds and ' $\Delta \mathrm{m}$ ' is the difference in $\mathrm{m} / \mathrm{z}$ values between the two peaks. If two peaks at a nominal $\mathrm{m} / \mathrm{z}$ of 100 are observed on separate channels of an oscilloscope sweep and offset by 0.100 mass units then the resolving power of the mass spectrometer would be 1,000 (100/0.1)[118]. Measuring peak separation can be done using the peak width definition method or the valley width definition method. According to the peak width definition, the value of $\Delta \mathrm{m}$ is the width of the peak measured at a designated peak height level and $\mathrm{m}$ is again the $\mathrm{m} / \mathrm{z}$ value of the peak.

The valley width definition method evaluates resolution by calculating the degree of overlap between two peaks. This method defines $\Delta \mathrm{m}$ as the closest possible spacing of two peaks of equal intensity where the height of the valley between them is less than a certain specified fraction of the peak height. When calculated using this method, resolution is generally reported as a certain resolving power with a percentage valley definition. If we take the previous example of a mass spectrometer where $\mathrm{m} / \Delta \mathrm{m}$ is equal to 1,000 and we find that the height of the valley 
between the two peaks is $10 \%$ of the peak height, the mass spectrometer has a resolving power of 1,000 with a $10 \%$ valley definition[118].

The ability of a quadrupole device to resolve different masses depends on several factors including the shape and diameter of the rods, the applied RF/DC voltages and the kinetic energy of ions entering and exiting the quadrupole[161]. Figure 6 in section 2.9.1 shows a Mathieu stability diagram of two masses entering a quadrupole at the same time. The three dotted lines passing through the plot represent RF/DC scan rates and while all three lines pass through regions of stable trajectory for both masses, resulting signals and ion abundances will be significantly different for each scan. The top line represents a high resolution scan as resulting spectral peaks will be narrow and well separated. If the mass range is scanned according to the middle line, peaks will be much wider and poorly separated resulting in decreased resolving power. Finally, if the mass range is scanned according to the bottom line, the spectral peaks will overlap and pass through the quadrupole without being separated. This results in inadequate resolution due to significant overlap of the spectral peaks[161].

It can also be seen in Figure 6 that the slope of the RF/DC scan rate can have a significant effect on sensitivity. The sensitivity of a certain scan rate for an ion of interest depends on how many of the variable RF/DC conditions fall within the regions of stability for that ion. If we again use Figure 6 as an example, it can be seen that while the top scan line represents a high resolution scan, the total number of ions that reach the detector will be quite low due to the minimal number of RF/DC combinations corresponding to stable trajectory. Conversely, the $\mathrm{RF} / \mathrm{DC}$ scan slope represented by the bottom line will provide greater sensitivity as more area of the stable trajectory curve is covered by the scan, however, spectral peaks will be wide and 
poorly separated. Because of this phenomenon, increased resolution is always accompanied by a sacrifice in sensitivity when using quadrupole mass filters.

\subsection{Waters TQD}

The Waters TQ Detector is a tandem quadrupole, atmospheric pressure ionization (API) mass spectrometer designed specifically for tandem mass spectrometric analyses following ultra performance liquid chromatographic (UPLC) separations[163]. The TQ Detector has been designed for compatibility with the ACQUITY UPLC ${ }^{\text {TM }}$ system and can be employed for both qualitative and quantitative analyses. The Waters TQD utilizes technological advances made in ion source configuration, T-Wave $\mathrm{TM}^{\mathrm{TM}}$ collision cell technology and data acquisition speed to take full advantage of the UPLC separation. The TQ detector utilizes a Z-Spray dual orthogonal ion source which acts to protect critical source and analyzer elements from the harmful effects of contamination by non-ionic species in complex biological matrices such as meconium (Figure 12)[171].

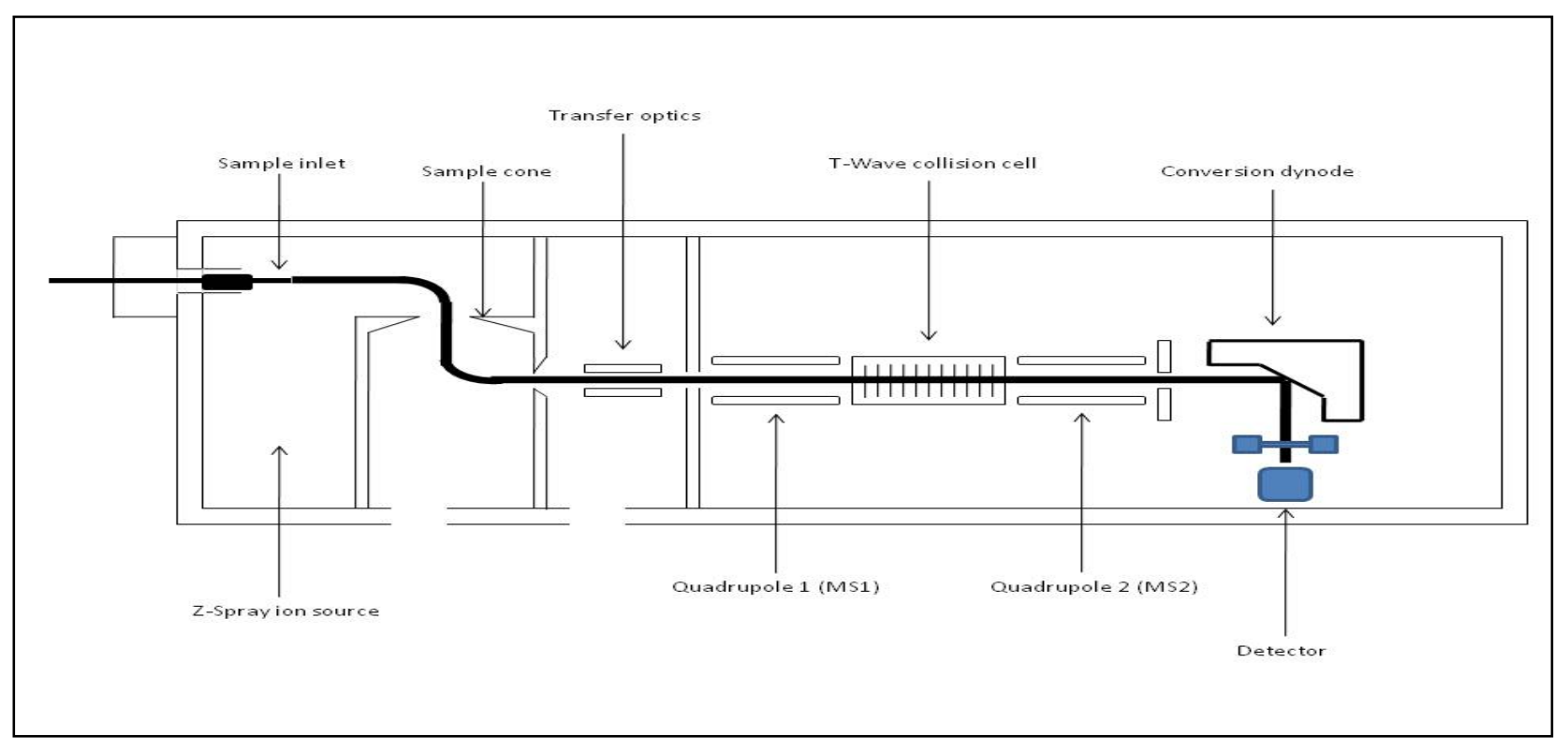

Figure 12: Schematic representation of the Waters TQD ion optics. 
Dual orthogonal sampling achieved with the Z-spray technique utilizes the same principles as conventional electrospray sources except that the trajectory of ions entering the analyzer is not a straight line, rather an approximate Z-shape[172](Figure 12). This trajectory deflects many neutral molecules which can build up on the orifice of skimmers in conventional sources. The final skimmer in the Z-spray ion source is set off to one side eliminating much of this material build up[172]. Protection of the source from matrix contamination through dual orthogonal sampling provides inherent sensitivity by not only decreasing background noise but also allowing for an increase in cone orifice size. Decreased background noise is achieved by the more effective removal of non-ionized contaminants by the dual orthogonal sampling technique. Due to the more effective removal of matrix contaminants, the sampling size of the cone orifice can be increased to allow more analyte ions to pass through to the mass spectrometer. By decreasing the background noise and increasing the number of analyte ions reaching the mass spectrometer, the Z-Spray dual orthogonal ion source ensures inherent sensitivity. The detection system has also been redesigned to accommodate the narrow ( $2-3$ seconds) peaks typical of UPLC separations. Accurate quantitation of such narrow peaks demands fast acquisition speeds to ensure that sufficient data points are obtained across each chromatographic peak. Failure to couple the UPLC with a detection system capable of rapid acquisition would result in the inability to accurately characterize chromatographic peaks. Table 5 outlines select system specifications of the TQD. 
Table 5: Select system specifications of the Waters triple quadrupole detector (TQD).

\begin{tabular}{|l|l|}
\hline \multicolumn{1}{|c|}{ System specification } & \multicolumn{1}{c|}{ Details } \\
\hline Acquisition modes & $\begin{array}{l}\text { Full scan; Selected ion recording (SIR); Product ion scan; } \\
\text { Precursor ion scan; Constant neutral loss/gain; Multiple reaction } \\
\text { monitoring (MRM) }\end{array}$ \\
\hline Mass range & 2 to $2000 \mathrm{~m} / \mathrm{z}$ \\
\hline Scan speed & Up to $10,000 \mathrm{Da} / \mathrm{s}$ \\
\hline MRM acquisition cycle time & Minimum dwell time of 5 ms per channel \\
\hline Mass resolution & $\begin{array}{l}\text { Valley between 2034.63 Da and 2035.63 Da peaks is }<12 \% \text { of } \\
\text { the average height of the two peaks }\end{array}$ \\
\hline
\end{tabular}

\subsection{Application of Tandem Mass Spectrometry in Forensic Toxicology}

The fields of forensic and clinical toxicology require confirmatory testing techniques which are capable of providing the most reliable and accurate information possible. The importance of superior selectivity, sensitivity, precision, robustness and speed may be more pronounced in the fields of forensic and clinical toxicology than any other. Analytical methodologies employed for the purposes of identifying and quantifying analytes in these fields need offer the highest selectivity and sensitivity as analyses commonly involve the determination of trace level xenobiotics in complex biological matrices rich in interferences.

The hyphenation of gas chromatography with mass spectrometry (GC/MS) has been widely employed in forensic and clinical laboratories worldwide since its introduction in the mid-twentieth century. The separation power, speed and cost effectiveness of gas chromatography, combined with the sensitivity and selectivity of mass spectrometry alleviated many of the problems associated with the detection of xenobiotics in complex samples of biological origin. GC/MS has long been the most widely employed technique for the selective determination and accurate quantification of drugs and their metabolites in biological 
samples[120]. Review of the scientific literature indicates a significant shift in the popularity and employment of confirmation techniques from largely GC/MS based methodologies to LC-MS and LC-MS/MS assays.

Early studies into the applications of tandem mass spectrometry were published by pioneering research groups such as Cooks and colleagues at Purdue University[173], Hunt and colleagues at the University of Virginia[174], and Enke et al at Michigan State University[175], however much of the earlier work utilized the selectivity and sensitivity of MS/MS without prior chromatographic separation. Reports of liquid chromatography-tandem mass spectrometry methodologies for the selective and sensitive determination of drugs in biological matrices consistently began to appear in the literature in the mid 1990s as a direct result of advances made in the design and commercial availability of sophisticated LC-MS interfaces.

Verweij and colleagues described a quantitative LC-MS/MS methodology for the determination of some analgesics and tranquilizers of the methadone, butyrophenone, or diphenylbutylpiperidine groups in whole blood. The authors employed a SRM mass spectrometric technique to monitor daughter ions produced by CID following thermospray (TS) ionization[176]. The same authors described a LC-MS/MS technique employing thermospray ionization for the quantitative analysis of several drugs with hypnotic, sedative and tranquillizing properties in whole blood in the following year. Quantitation was performed using a triple quadrupole mass spectrometer operating in the SRM mode following HPLC separation. The authors report detection limits in the range of $0.05-0.5 \mathrm{ng} / \mathrm{mL}$ for most analytes[177].

The potential of the atmospheric pressure ionization techniques was realized from the beginning and the mid 1990s saw a large increase in the number of published applications while alternative ionization techniques such as thermospray, fast atom bombardment, and particle 
beam ionization began to disappear from the literature[131].Cai and Henion employed a triple quadrupole mass spectrometer interfaced with an electrospray ionization source for the trace analysis of LSD and analogues in human urine following on-line immunoaffinity extraction. Coupling of the on-line extraction to the tandem mass spectrometric analysis provided the Cai and Henion with detection limits as low as $2.5 \mathrm{ppt}$ which represented a 20 fold increase in sensitivity relative to their previous solid phase extraction technique[178]. Cai and Henion also describe a technique for the elucidation of LSD metabolism pathways in vitro by employing the precursor and neutral loss scanning modes of the triple quadrupole mass spectrometer (see section 2.9.4) following separation by LC and capillary electrophoresis. Although the precursor and neutral loss scanning modes employed here are seldom used for the quantitation of target drugs, Cai and Henion were able to positively identify two new in vitro metabolites of the hallucinogen whose structures were unequivocally established through comparisons with reference standards[179]. Constanzer and colleagues employed a triple quadrupole mass spectrometer interacted with positive ion APCI for the determination of cyclobenzaprine in human plasma and urine. The sensitivity of the LC-MS/MS method was compared to a previously established GC/MS method and a HPLC separation employing UV detection. The authors concluded that the LC-MS/MS technique allowed for a ten-fold increase in sensitivity relative to the GC method and a five-fold increase in sensitivity relative to the HPLC-UV methodology[180]. Kleinschnitz and co-workers also employed APCI interfaced with a triple quadrupole mass spectrometer for the analysis of 1,4-benzodiazepines in serum and urine allowing detection quantification limits of $2 \mathrm{ng} / \mathrm{mL}[181]$.

In the years following these early studies, reported applications of LC-MS/MS, in particular, liquid chromatography coupled to triple quadrupole instruments, for the determination 
of drugs or toxins in sample of biological origin increased exponentially. The current role of LCMS/MS in forensic and clinical toxicology has been the focus of several reviews $[120,131,182]$. Several LC-MS/MS techniques with forensic significance are reviewed here, focusing primarily on the detection and/or quantification of drugs of abuse in biological samples.

Hegstad and co-workers validated a triple quadrupole mass spectrometry method employing positive ion mode ESI for the purpose of screening nicotine, cotinine, morphine, 6monoacetylmorphine, codeine, amphetamine, methamphetamine, 3,4-methylenedioxymethamphetamine, cocaine, benzoylecgonine, 7-aminonitrazepam, 7-aminoclonazepam, 7aminoflunitrazepam, oxazepam, diazepam, alprazolam, zopiclone, zolpidem, carisoprodol, meprobamate, buprenorphine, and methadone in hair samples. Following validation, the ESIMS/MS method was implemented for the analysis of authentic samples[183]. Concheiro et al recently employed positive mode electrospray chemical ionization (ESCI) interfaced with a triple quadrupole mass spectrometer for the determination of morphine, codeine, 6monoacetylmorphine, methadone, amphetamine, methamphetamine, 3,4methylenedioxyamphetamine, 3,4-methylenedioxymethamphetamine, 3,4-methylenedioxy-Nethylamphetamine, benzoylecgonine, cocaine, delta-9-tetrahydrocannabinol, zolpidem, zopiclone, alprazolam, clonazepam, oxazepam, nordiazepam, lorazepam, flunitrazepam, diazepam, diphenhydramine and amitriptyline in preserved oral fluid specimens. Detection limits of $0.5 \mu \mathrm{g} / \mathrm{L}$ were achieved using the tandem-in-space mass spectrometry method[184]. Gaulier et al reported a tandem mass spectrometry method for the sensitive determination of acepromazine for the purpose of identifying drug facilitated sexual assault (DFSA) crimes. The authors employed a TSQ tandem mass spectrometer equipped with an orthogonal electrospray ionization 
source and determined the presence of acepromazine in a sample of the victims hair at a concentration of $31 \mathrm{pg} / \mathrm{mg}[185]$.

Ojanpera and co-workers reported a sensitive tandem mass spectrometry technique for the detection of the highly potent opioid designer drug 3-methylfentanyl (TMF) in post-mortem specimens. Analysis was performed using a QTrap LC-MS/MS operated in triple quadrupole mode allowing for the unequivocal identification of the drug at lethal levels in 117 cases over a two year period[186]. Roman et al investigated the potential of tandem mass spectrometry following liquid chromatographic separation for the detection of seven antipsychotic drugs commonly dosed at low levels. Analysis was performed on a triple quadrupole instrument equipped with an electrospray interface and the authors conclude that the enhanced sensitivity and selectivity afforded by LC-MS/MS is fast becoming essential for the determination of such drugs to properly assess their role in post-mortem cases[187].

Liu et al describe a method employing the fast scanning capabilities of a triple quadrupole mass spectrometer operating in the MRM mode for the purpose of screening 22 poisonous alkaloids in human blood. Positive ion ESI followed by MS/MS analysis enabled the authors to selectively identify 22 compounds in human blood based on two fragmentation transitions for each analyte. The authors achieved detection limits in the range of $0.1 \mathrm{ng} / \mathrm{mL}$ to 20ng/mL[188]. Concheiro and co-workers utilized a triple quadrupole mass spectrometer equipped with a Z-spray ion source for the simultaneous determination of ecgonine methyl ester, benzoylecgonine, morphine, codeine, 6-acetylmorphine, amphetamine, methamphetamine, 3,4methylenedioxyamphetamine (MDA), 3,4-methylenedioxymethamphetamine (MDMA), methadone, 2-ethylidene-1,5-dimethyl-3,3-diphenylpyrrolidine (EDDP), and d-lysergic acid diethylamide (LSD) in human urine and highlighted the importance of ion ratios in the 
identification criteria. The authors suggest that additional research efforts should be focused on determining satisfactory variations in ion ratios following fragmentation by the softer LCMS/MS ionization techniques[189].

Castaing and co-workers capitalized on the sensitivity, selectivity and multi-analyte detection capabilities of a triple quadrupole mass spectrometer equipped with an ESI source to simultaneously screen and quantify selective serotonin reuptake inhibitors (SSRIs) (fluoxetine, paroxetine, sertraline, fluvoxamine, and citalopram), serotonin noradrenergic reuptake inhibitors (SNaRIs) (milnacipram and venlafaxine), mirtazapine and five of their active metabolites in whole blood. The authors describe the first method in the literature for the simultaneous screening and quantification of all aforementioned drugs using LC-MS/MS and highlight that the more traditional analytical techniques lack the capability to detect and quantify drugs from the SSRI, SNaRI and related classes simultaneously[190].

Castro and co-workers successfully combined a solid phase extraction and LC separation into a single system to allow for the direct injection of plasma samples for the simultaneous determination of 14 antidepressants and their metabolites by positive ion ESI and tandem mass spectrometry. The authors' state that the minimal sample preparation required for LC-MS/MS analysis, its high selectivity and sensitivity in combination with good precision and accuracy over a wide dynamic range, allow for the development of much more efficient and rapid analytical methodologies. In particular the authors state that the application of LC-MS/MS for the separation and detection of multiple antidepressants in a single sample eliminates the need for lengthy sample preparation steps such as fluorescence derivatization which is required for the more traditional HPLC with fluorescence detection approach[191]. 
The enhanced sensitivity of tandem mass spectrometry was employed by Quintela et al for the detection of low concentrations of 21 benzodiazepine, metabolites and analogues in urine using a triple quadrupole mass spectrometer equipped with an ESI interface. Successful detection was achieved by monitoring two fragmentation transitions for each analyte in the MRM mode. The authors conclude that the increased sensitivity afforded by tandem mass spectrometry assists in the detection of low concentrations of benzodiazepines used in drug facilitated sexual assault crimes[192].

Almost 15 years on from the first reported applications of API techniques for the ionization of analytes prior to tandem mass spectrometric analysis, ESI and APCI find widespread use in routine toxicological analysis worldwide[120, 131, 182]. Just as API techniques have become common practice in toxicological analysis, triple quadrupole mass spectrometers have been the focus of the vast majority of studies involving the detection and quantitation of xenobiotics in toxicological specimens. Tandem mass spectrometry employing hybrid mass analyzers for the purpose of qualitative and/or quantitative determination of xenobiotics in toxicological specimens became the focus of many research groups in the early twenty first century.

Ballard and co-authors described a novel analytical strategy for the analysis of quaternary ammonium neuromuscular blocking agents in a wide variety of biological specimens using a tandem quadrupole orthogonal acceleration time of flight (Q-TOF) instrument. The authors exploit the qualitative power of the modern quadrupole/time-of-flight (Q-TOF) mass spectrometer by using the full scan product-ion spectra to unequivocally identify several neuromuscular blocking agents at levels as low as $2-10 \mathrm{ng} / \mathrm{g}$ in various biological matrices. Concluding remarks highlight the superiority of the modern Q-TOF instrument relative to the 
commonly employed tandem quadrupole for forensic analyses in which qualitative findings are of the utmost importance[193].

Frison et al recently employed an ion trap mass spectrometer equipped with an electrospray ionization source (ESI) for the sensitive determination of citalopram and its (S) enantiomer, escitalopram, along with their demethylated metabolites in neonatal hair samples. The authors reported a quantification limit of $25 \mathrm{pg} / \mathrm{mg}$ for all analytes using the tandem-in-time mass spectrometry approach[194].

Deviation from conventional tandem quadrupole mass spectrometers for forensic screening and confirmation was also demonstrated by Sauvage and co-workers who developed a comprehensive screening method for 1250 exogenous compounds in human blood and urine using a triple quadrupole linear ion trap mass spectrometer. The authors developed a general unknown screening procedure based on linear ion trap MS/MS using advanced computer programming capabilities for the analysis of clinical and forensic samples. By storing product ions in the linear ion trap following CID, the authors demonstrated superior identification capabilities in a direct comparison with GC/MS and HPLC-DAD[167]. Favretto et al employed an ion trap mass spectrometer for the sensitive determination of buprenorphine and norbuprenorphine in urine, blood and hair samples and comparatively investigated the use of an ion trap for the purposes of collision induced dissociation rather than the in-source fragmentation techniques previously reported in the literature. The authors report a much higher CID efficiency through the use of an ion trap which appears to generate not only more product ions but also a higher abundance of each. Collective data suggests that ion trap MS/MS experiments for the determination of buprenorphine and its active metabolite are not only superior to the previously reported LC/MS experiments employing in-source collisions and a single quadrupole, but also 
offer enhanced sensitivity relative to triple quadrupole mass spectrometers employing a CID cell. Variations in sensitivity between the two instruments were attributed to the high collision energies required to produce any appreciable fragmentation in the triple quadrupole CID, combined with the losses in sensitivity associated with ion transfer through tandem-in-space instruments[195].

In 2005, Herrin and co-workers utilized the large database of multiple reaction monitoring transition ions for drugs amendable to LC/MS/MS to create a tandem mass spectrometry technique for the screening of drugs in post mortem specimens. Herrin et al employed a QTrap LC-MS/MS for the purpose of unequivocally identifying over 100 drugs, many of which are not amendable to enzyme immunoassay (EIA) screening techniques. Qualitative determinations were made based on the detection of MRM transition ions and the enhanced product ion (EPI) scans produced by the linear ion trap. Enhanced product ion scans are produced when Q3 is employed as a linear ion trap while simultaneously performing the role of Q3 for the MRM experiments. Identifications based on the detection of one MRM transition ion and a matching EPI scan enabled the authors to unequivocally identify over 100 drugs in post-mortem specimens using dwell times in the range of 1-25 ms[168].

Prior to the studies of Herrin et al, several groups published methodologies describing the application of quadrupole-linear ion-trap mass spectrometry for the broad based screening of forensically significant drugs in post-mortem specimens[167, 169, 196, 197]. The number of published methodologies describing the use of liquid chromatography coupled to tandem mass spectrometry for the forensic analysis of drug or other xenobiotics continues to increase each year as the instrumentation capable of performing LC-MS/MS analysis continues to become more affordable and intuitive (Figure 13). 


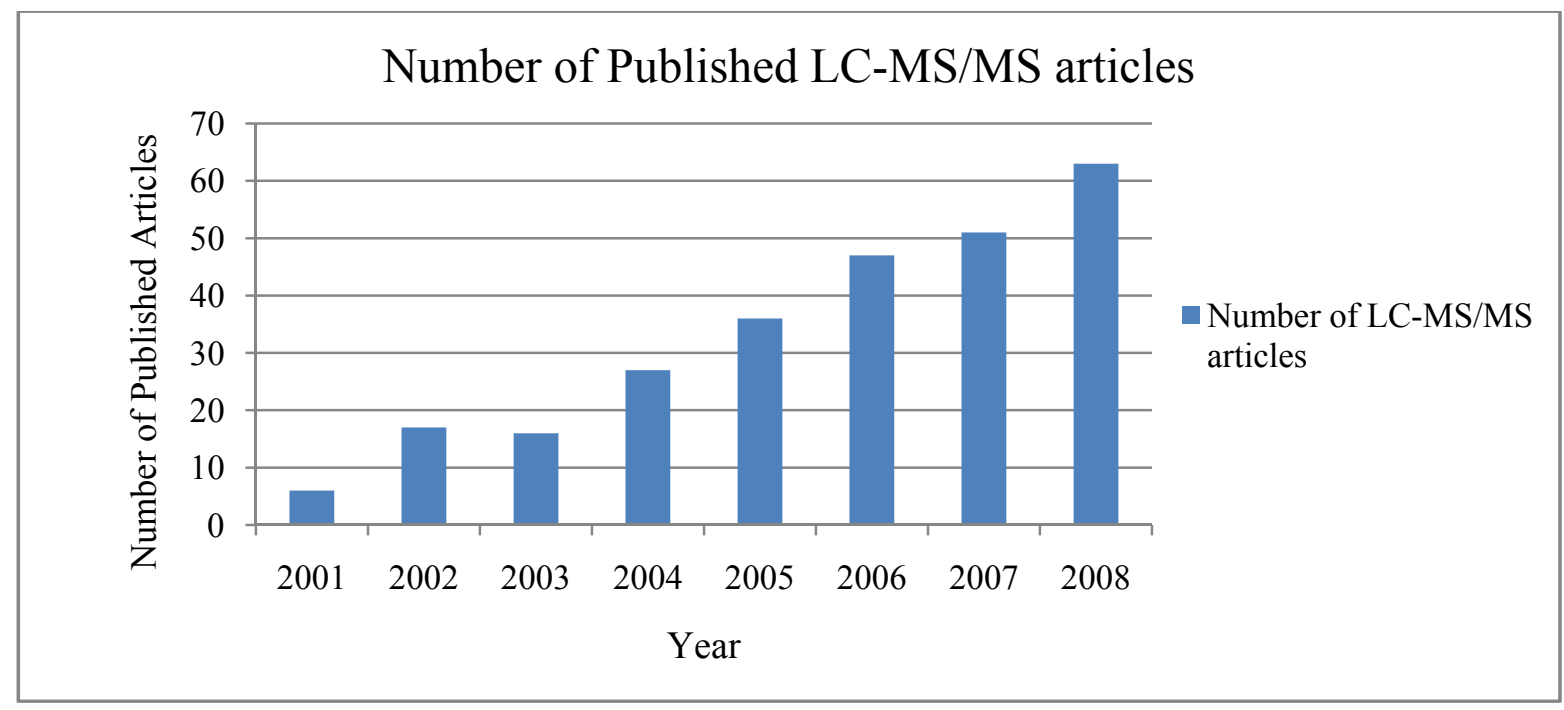

Figure 13: Number of LC-MS/MS articles appearing in pubmed from 2001 - 2008 for the forensic analysis of drugs or other xenobiotics. 


\section{Chapter 3: Analytical Method Validation}

\subsection{Introduction}

Analytical methodologies play an integral role in many fields of the life sciences and as a result, produce data that is subsequently interpreted to provide pivotal information which is then used to make medical, legal, clinical, and environmental decisions. Correct interpretation and evaluation of analytical data is obviously a crucial component in the decision making process, however, preliminary procedures must also be implemented by analytical chemists to ensure that such methodologies are generating reliable analytical data at all times. Analytical data of high quality and integrity can only be produced by an analytical method which has demonstrated both suitability and reliability for its intended purpose. Careful method development followed by a thorough bioanalytical method validation is required for analytical methodologies whose intended use is to quantify the concentration of an analyte in a particular biological matrix for the purpose of forensic and/or clinical toxicology analysis[198].

Bioanalytical method validation for analytical techniques intended for forensic toxicology analysis is of extreme importance as it objectively demonstrates the inherent quality of the analytical method for its intended purpose by fulfilling minimum performance and acceptance criteria[198]. Failure to validate a bioanalytical methodology for its intended purpose may unknowingly generate unreliable data which will not only be questioned in a court of law, but may result in unjustified legal consequences or the inappropriate treatment of patients[198]. Unreliable data generated during analytical studies to assess the pharmacological efficacy of a new drug or patient compliance in pain management toxicology can have detrimental effects on both the patient and the toxicology laboratory responsible for generating the data. All appropriate 
steps must be taken to ensure that the data generated by bioanalytical methodologies in the forensic toxicology laboratory is both accurate and reliable.

The first step in validating a bioanalytical method is to identify and define its intended purpose as this will largely determine which method parameters require validation. As there is no one single guideline on bioanalytical method validation, it is routinely customized by individual laboratories by the identification and validation of only select performance criteria which have been chosen based largely on the intended purpose of the test. Selection of relevant performance criteria is made easier by first identifying the intended purpose or application of the method. For example, certain validation parameters such as precision and accuracy are vital performance criteria which need be addressed during the validation of a quantitative bioanalytical method whereas validation of certain qualitative assays may not incorporate the assessment of such parameters. Bioanalytical method validation procedures show significant variation from laboratory to laboratory and although there is no single guideline describing the processes for full method validation, several guidance documents addressing the bioanalytical analysis of drugs and poisons in biological samples exist in the literature [119, 198-202]. In the present study, analytical methodologies were developed for the purpose of quantifying drugs of abuse in meconium. All analytical method validation protocols were designed and followed according to validation guidelines set out in documents such as the bioanalytical method validation guidance for industry document published by the US Food and Drug Administration in 2001, and the Forensic Toxicology laboratory guidelines published by the Society of Forensic Toxicologist (SOFT) and American Academy of Forensic Sciences (AAFS).

Full bioanalytical validations should be completed when developing and implementing a method for the first time or if the method is intended for the detection of drugs and/or 
metabolites in novel matrices. Guidance presented in these documents applies to the validation of bioanalytical procedures such as gas chromatography (GC), high-pressure liquid chromatography (LC), combined GC and LC mass spectrometric procedures such as LC-MS, LC-MS/MS, GCMS, and GC-MS/MS performed primarily for the quantitative determination of drugs and their metabolites in various biological matrices such as blood, serum, plasma, or urine.

The present study describes the development of three UPLC-MS/MS methodologies for the quantitative determination of several drugs of abuse in human meconium. Implementation of this technique for the intended purpose has not occurred previously and because of this, all three UPLC-MS/MS methodologies were subjected to full analytical method validation procedures.

Fundamental parameters for a full bioanalytical method validation include accuracy, precision, selectivity, sensitivity, reproducibility, and stability. Method validation was performed for each confirmation assay by determining and assessing (1) selectivity, (2) accuracy, (3) precision, recovery, (3) calibration curve, (4) Limit of detection, and (5) stability. Validation of each analytical method involved documenting the performance of each of these criteria during laboratory experiments designed specifically for that purpose. Each performance parameter was designated certain acceptance criteria which had to be satisfied in order to deem the method suitable and reliable for its intended purpose. Table 6 provides an overview of bioanalytical method validation terms, abbreviations, and definitions. 
Table 6: Overview of bioanalytical method validation terms and definitions.

\begin{tabular}{|l|l|}
\hline \multicolumn{1}{|c|}{ Term } & \multicolumn{1}{|c|}{ Definition } \\
\hline Selectivity & $\begin{array}{l}\text { The ability of an analytical method to differentiate and quantify the } \\
\text { analyte of interest in the presence of other components in the sample }\end{array}$ \\
\hline Accuracy & $\begin{array}{l}\text { The closeness of mean test results obtained by the method to the true } \\
\text { concentration of the analyte }\end{array}$ \\
\hline Precision & $\begin{array}{l}\text { The closeness of individual measures (degree of scatter) of an analyte } \\
\text { concentration when the procedure is applied repeatedly to multiple } \\
\text { aliquots of a single standard }\end{array}$ \\
\hline Recovery & $\begin{array}{l}\text { Recovery pertains to the extraction efficiency of an analytical method } \\
\text { within the limits of variability }\end{array}$ \\
\hline Calibration curve & $\begin{array}{l}\text { Represent the relationship between instrument response and known } \\
\text { concentrations of an analyte }\end{array}$ \\
\hline Stability & $\begin{array}{l}\text { Pertains to the stability of an analyte in a biological matrix during sample } \\
\text { collection, and storage }\end{array}$ \\
\hline Limit of detection (LOD) & $\begin{array}{l}\text { The lowest concentration or amount of analyte in a sample that can be } \\
\text { unequivocally identified but not necessarily quantified }\end{array}$ \\
\hline Lower limit of quantitation (LLOQ) & $\begin{array}{l}\text { The lowest concentration of an analyte in a sample that can be quantitated } \\
\text { with suitable accuracy and precision }\end{array}$ \\
\hline
\end{tabular}

\subsection{Bioanalytical Validation Parameters}

\subsubsection{Selectivity}

Selectivity is the ability of an analytical method to differentiate and quantify the analyte of interest in the presence of other components in the sample[199]. Selectivity is an extremely important validation parameter in forensic toxicology due to the complex nature of biological specimens such as blood, urine, tissue, and meconium. Complex matrices such as meconium are expected to be rich in interfering species of both endogenous and exogenous origin. This means that bioanalytical methods intended to quantify small amounts of analyte, must be capable of unequivocal identification and accurate quantification in the presence of additional sample 
components such as xenobiotics, metabolites, impurities, degradants, and endogenous interferences[198].

Chemical extractions are routinely employed in forensic toxicology to aid in the removal of interfering species from the biological matrix prior to instrumental analysis. While various chemical extractions (SPE, LLE, protein precipitations) have been shown to selectively extract common drugs of abuse while simultaneously removing matrix components, many of these techniques are validated only for blood or urine. Meconium presents a significantly different matrix of biological origin and the application of pre-existing extraction techniques may not necessarily provide extracts of the same purity.

Selectivity studies for alternate matrices such meconium, are important for determining whether or not the chosen chemical extraction is suitable and sufficient. To determine the extent of sample cleanup, and therefore the suitability of the extraction technique, multiple ( $\mathrm{n}=5$ ) blank matrices should be subject to chemical extraction and analyzed for the analyte(s) of interest. Any analyte response generated by endogenous compounds in the blank matrix should correspond to an analyte concentration below the LLOQ. Five blank biological matrices should be subject to chemical extraction and analyzed for the analyte of interest to ensure that any analyte response generated by endogenous compounds could not result in a confirmed positive result.

Selectivity of the instrumental detection system is also paramount to ensure that the analyte(s) of interest are accurately identified in the presence of chemically similar exogenous interferences. Chemical extractions are often sufficient for the removal of endogenous compounds which are chemically and physically distinct from the analyte(s) of interest, however, xenobiotics with similar physicochemical properties will likely not be removed during chemical extraction. For this reason, detection systems must be capable of accurate and unequivocal 
analyte identification in the presence of chemically similar compounds. Mass spectral characteristics of chemically similar compounds commonly encountered in forensic specimens should be studied to ensure that they are distinguishable from the analyte of interest. In order to evaluate the selectivity of the methodology, several blank matrices should be spiked with the analyte of interest at a concentration corresponding to the LLOQ and also with various exogenous interferences. Samples should be analyzed to ensure that the methodology is capable of accurately identifying and quantifying the analyte of interest in the presence of chemically similar compounds.

\subsubsection{Accuracy}

The accuracy of an analytical methodology describes the closeness of mean test results obtained by the method to the true concentration of the analyte[199]. The accuracy of an analytical methodology should be determined by preparing and analyzing five replicate standards in the matrix under investigation at three concentrations spanning the calibration range of the method. Five standards containing a known amount of the analyte should be prepared at concentrations representing the upper limit of quantitation (ULOQ), the LOQ, and a concentration point midway between the two. Replicate analysis of the standards should produce mean values within $15 \%$ of the expected nominal value, except at the LLOQ where the mean value should be within $20 \%$ of the expected nominal value. The degree of deviation of mean values from the true values serves as the measure of method accuracy[199].

\subsubsection{Precision}

The precision of an analytical method describes the closeness of individual measures (degree of scatter) of an analyte concentration when the procedure is applied repeatedly to multiple aliquots of a single standard[199]. The precision of an analytical methodology should be 
determined by preparing and analyzing five replicate standards in the matrix under investigation at three concentrations spanning the calibration range of the method. Precision of the analytical method should not exceed $15 \%$ of the coefficient of variation (CV) except at the LLOQ where is should not exceed $20 \%$ of the CV. Precision studies can be further subdivided into intra-batch precision and inter-batch precision. Intra-batch precision evaluates the method precision during a single analytical run or single batch. Inter-batch precision evaluates the method precision over multiple batches and provides an indication of method repeatability with time. Inter-batch precision experiments may be as simple as analyzing replicates under identical conditions over several days or may involve using different analysts, instruments, reagents, or laboratories to determine the robustness of the methodology. Mathematically determining the precision of replicate analysis at any one concentration involves calculating the standard deviation of the replicate concentrations and dividing by their mean.

\subsubsection{Recovery}

Recovery of an analytical methodology should be evaluated to determine the extraction efficiencies for each analyte from the matrix of interest. Recovery pertains to the extraction efficiency of an analytical method within the limits of variability. Recovery is not among the validation parameters regarded as essential for quantitative assays unless the sensitivity of the assay is poor[198].

Analyte recovery pertains to the detector response obtained from a known amount of analyte added to and then extracted from a biological matrix, compared to the detector response obtained for the same concentration analyzed as a pure authentic standard[199]. While the recovery of an analyte and its respective internal standard need not be $100 \%$, the extent of recovery should be reproducible at concentrations spanning the calibration range. 


\subsubsection{Calibration Curves}

Calibration curves consist of a number of standards prepared at known concentrations in the same matrix as the unknown specimens. Following accurate preparation, calibration standards are analyzed to determine how the instrumental response changes with analyte concentration. Once this relationship has been established, unknown samples can be analyzed and quantified by measuring the analyte response and interpolating to find the concentration of the analyte. Calibration curves are the most commonly employed internal standard method for forensic toxicological analysis. Preparation of calibration curve standards in appropriate matrices for the purpose of quantifying unknown analytes is typical across toxicology laboratories. Calibration curves should be generated and assessed for each analyte encompassed in the analytical method. A sufficient number of calibrator concentrations should be employed to cover the entire calibration range and should be evenly spaced to accurately and adequately define the relationship between analyte concentration and detector response[119, 198, 199].

Calibration standards used to construct calibration curves should be constructed from blank biological matrices which have been spiked with a known amount of analyte and internal standard. Detector responses are generally calculated as the area ratio of analyte versus internal standard for bioanalytical methods[198].

\subsubsection{Stability Studies}

Analyte stability in a biological matrix is a function of the storage conditions, the physicochemical properties of the drug itself, and the matrix in which the drug is present[199]. Stability studies should be performed to assess the stability of each analyte in the matrix of interest and in the storage container to be used. Stability studies are specific to the matrix, storage container, storage conditions, and the analyte investigated during validation studies and 
results should not be extrapolated or applied to other matrices or conditions. Stability studies should be designed to evaluate the stability of each analyte during collection, pre-testing storage, analysis procedures, and post-testing storage. Studies should also be designed to investigate analyte stability during and after several freeze and thaw cycles. Stability of the analyte following several freeze-thaw cycles is important to establish as samples are routinely subject to such conditions during retesting. Investigations into analyte stability should be performed by spiking the appropriate blank matrix with each analyte at concentrations within the expected range. Samples prepared at a high and low concentration relative to the calibration curve are most appropriate. Spiked samples should then be subject to various conditions representative of sample collection, storage and preparation. Samples should also be subject to several freeze-thaw cycles and analyzed following each cycle to ensure stability over the time course. Mean values obtained after subsequent freeze-thaw cycles should be within $20 \%$ of the expected nominal values if the analytes are indeed stable in the matrix of interest. The stability of analytes and internal standards during and prior to analysis, but following extraction may also be investigated. These studies are important for high throughput production laboratories as samples are often batched and loaded into sample organizers for subsequent analysis. If multiple batches are to be analyzed over a significant period of time, prepared extracts may experience significant residence time in the autosampler. Stability of the analyte in such an environment should be established in such cases.

\subsubsection{Limit of Detection (LOD) and Lower Limit of Quantitation (LLOQ) Studies}

The limit of detection (LOD) for an analytical methodology is defined as the lowest concentration or amount of analyte in a sample that can be unequivocally identified but not necessarily quantified. It corresponds to the lowest concentration of analyte that the analytical 
methodology can reliably differentiate from background noise[201]. The LOD can be determined either by a statistical approach based on measuring replicate blank (negative matrix) samples or by an empirical approach which consists analyzing progressively more dilute concentrations of analyte until reliable identification can no longer be made[203]. Statistical determination of the LOD is achieved by analyzing a series of blank samples (appropriate matrix with no analyte) and obtaining the mean blank value $(\mu)$ and the standard deviation $(\sigma)$ of those values. The LOD is then determined using Equation 11.

$\mathrm{LOD}=\mu+3 \sigma$

Equation 11

The statistical approach assumes that the standard deviation of the blank is roughly equivalent to the standard deviation of the concentration that actually corresponds to the LOD[203]. Similar to the statistical approach is the method for determining the LOD based on the signal to noise $(\mathrm{S} / \mathrm{N})$ ratio. The signal to noise is defined as the height of the analyte peak (signal) and the amplitude between the highest and lowest point of the baseline (noise)[198]. The LOD is normally accepted as the concentration at which the $\mathrm{S} / \mathrm{N}$ ratio is equal to, or greater than 3 for triplicate analyses. The empirical or experimental approach to determining the LOD involves analyzing serially dilute samples of analyte until the LOD is determined. The LOD corresponds to the lowest concentration of sample at which the identification of the analyte still satisfies predetermined acceptance criteria[203]. Concentrations below the LOD do not satisfy such criteria and identification of the analyte would not be justified. The limit of detection was determined empirically for all confirmation methodologies by analyzing serially dilute samples until the methodology was unable to reliably identify the analyte as a result of shifts in retention times or 
inaccurate ion ratios. Acceptance criteria for retention times were within $2 \%$ of calibrators and ion ratios were required to be within $20 \%$ of calibrators to positively identify the analyte. Evaluation and determination of the LOD for instrumental methods designed for forensic analysis may not always be necessary. While it is good practice to understand instrument limitations, forensic laboratories rarely report positive results at concentrations corresponding to the LOD. This is because most chromatographic methodologies employed in the toxicology laboratory are confirmatory assays aimed at quantitating an unknown analyte. As quantitation at the LOD is not feasible, many laboratories do not consider it a vital validation parameter. A more important validation parameter in the forensic toxicology industry is the lower limit of quantitation.

The lower limit of quantitation (LLOQ) is defined as the lowest concentration of an analyte in a sample which can be accurately and precisely quantitated[199]. The LLOQ is an important parameter in toxicology as all chromatographic assays are designed to accurately quantitate an unknown analyte. As accurate quantitation below the LLOQ is not feasible, any sample that contains an analyte below the established LLOQ is generally considered negative in the toxicology laboratory. There are several approaches to determining the LLOQ and the most appropriate depends largely on the intended purpose of the analytical methodology. The LLOQ can be determined statistically using the same technique as the LOD method and substituting $\mu$ and $\sigma$ into Equation 12.

$\operatorname{LLOQ}=\mu+10 \sigma$

Equation 12 
Similar to this approach is the method of determining the LLOQ based on the signal to noise $(\mathrm{S} / \mathrm{N})$ ratio. The signal to noise is defined as the height of the analyte peak (signal) and the amplitude between the highest and lowest point of the baseline (noise)[198]. The LLOQ normally corresponds to the concentration at which the $\mathrm{S} / \mathrm{N}$ ratio is equal or greater than 10 . Laboratory guidelines published by SOFT/AAFS state that for chromatographic assays, the LLOQ may be defined as the concentration of the lowest calibration standard. The LLOQ is the concentration at which the analytical methodology can still quantify the analyte with acceptable accuracy and precision. 


\section{Chapter 4: Introduction and Overview to the Development and Validation of UPLC-MS/MS Methodologies for the Quantitative Determination of Cocaine, Amphetamine, and Opiate Derivatives in Human Meconium.}

\subsection{Introduction}

Cocaine, amphetamines, and opiates continue to find widespread abuse among users worldwide. Due to the detrimental effects of prenatal drug exposure, there is a growing need for methodologies capable of assisting with the identification of maternal drug use. Traditional methods for identifying maternal drug use have relied on the analysis of maternal and/or infant blood and urine. Due to the relatively short retention time of drugs in blood and urine, these analyses aid only in the identification of recent maternal drug use. Development of sensitive and selective methodologies capable of detecting drugs of abuse in human meconium will aid in identifying prenatal drug exposure occurring during the second and third trimesters which has previously gone undetected. Implementation of such methodologies will ensure that the correct intervention and treatment is made available to both the mother and the newborn.

Development and full analytical method validation of three UPLC-MS/MS methodologies for the quantitative determination of cocaine, amphetamine, and opiate derivatives in human meconium is described. Methodologies were developed for the purpose of selectively determining and accurately quantifying cocaine, benzoylecgonine, amphetamine, methamphetamine, codeine, morphine, hydromorphone, and 6-monoacetylated morphine in meconium specimens. All experiments were performed using the Waters TQD triple quadrupole mass spectrometer equipped with an electrospray ionization (ESI) source operating in the positive ion mode. Chromatographic separations prior to mass spectrometric detection were achieved using the Waters ACQUITY UPLC ${ }^{\text {TM }}$ chromatograph. Methodologies were validated 
according to full analytical method validation guidelines published in the 'Bioanalytical Method Validation Guidance for Industry' document published by the US Food and Drug Administration in 2001, and the 'Forensic Toxicology Laboratory Guidelines' published by the Society of Forensic Toxicologist (SOFT) and American Academy of Forensic Sciences (AAFS)[119, 199]. Tables 7 provides an overview of the three analytical methodologies including the number of analytes detected by each methodology, sample preparation method, and figures of merit such as the calibration range, the limit of detection (LOD), and the lower limit of quantitation (LLOQ).

Table 7: Overview of three UPLC-MS/MS methodologies developed and validated for the purpose of detecting and quantifying several cocaine, amphetamine, and opiate derivatives in human meconium.

\begin{tabular}{|c|c|c|c|c|c|}
\hline $\begin{array}{c}\text { UPLC } \\
\text { methodology }\end{array}$ & Analyte & Sample preparation & $\begin{array}{l}\text { Calibration } \\
\text { range }\end{array}$ & $\overline{\text { LOD }}$ & LLOQ \\
\hline \multirow[t]{2}{*}{$\begin{array}{c}\text { Methodology } \\
\# 1 . \\
\text { Chapter } 5\end{array}$} & 1. Cocaine & $\begin{array}{l}\text { 1.Protein precipitation } \\
\text { 2. SPE }\end{array}$ & $10-250 \mathrm{ng} / \mathrm{mL}$ & $1 \mathrm{ng} / \mathrm{mL}$ & $10 \mathrm{ng} / \mathrm{mL}$ \\
\hline & 2. Benzoylecgonine & $\begin{array}{l}\text { 1.Protein precipitation } \\
\text { 2. SPE }\end{array}$ & $10-250 \mathrm{ng} / \mathrm{mL}$ & $1 \mathrm{ng} / \mathrm{mL}$ & $10 \mathrm{ng} / \mathrm{mL}$ \\
\hline \multirow{4}{*}{$\begin{array}{c}\text { Methodology } \\
\# 2 . \\
\text { Chapter } 6\end{array}$} & 1. Morphine & $\begin{array}{l}\text { Supported-liquid } \\
\text { extraction (SLE) }\end{array}$ & $10-500 \mathrm{ng} / \mathrm{mL}$ & $2.5 \mathrm{ng} / \mathrm{mL}$ & $10 \mathrm{ng} / \mathrm{mL}$ \\
\hline & 2. Codeine & $\begin{array}{l}\text { Supported liquid } \\
\text { extraction (SLE) }\end{array}$ & $10-500 \mathrm{ng} / \mathrm{mL}$ & $2.5 \mathrm{ng} / \mathrm{mL}$ & $10 \mathrm{ng} / \mathrm{mL}$ \\
\hline & 3. Hydromorphone & $\begin{array}{l}\text { Supported liquid } \\
\text { extraction (SLE) }\end{array}$ & $10-500 \mathrm{ng} / \mathrm{mL}$ & $2.5 \mathrm{ng} / \mathrm{mL}$ & $10 \mathrm{ng} / \mathrm{mL}$ \\
\hline & 4. 6-monoacetylmorphine & $\begin{array}{l}\text { Supported liquid } \\
\text { extraction (SLE) }\end{array}$ & $10-500 \mathrm{ng} / \mathrm{mL}$ & $2.5 \mathrm{ng} / \mathrm{mL}$ & $10 \mathrm{ng} / \mathrm{mL}$ \\
\hline \multirow{2}{*}{$\begin{array}{c}\text { Methodology } \\
\text { \#3. } \\
\text { Chapter } 7\end{array}$} & 1. Amphetamine & $\begin{array}{l}\text { Supported liquid } \\
\text { extraction (SLE) }\end{array}$ & $5-500 \mathrm{ng} / \mathrm{mL}$ & $1 \mathrm{ng} / \mathrm{mL}$ & $5 \mathrm{ng} / \mathrm{mL}$ \\
\hline & 2. Methamphetamine & $\begin{array}{l}\text { Supported liquid } \\
\text { extraction (SLE) }\end{array}$ & $5-500 \mathrm{ng} / \mathrm{mL}$ & $250 \mathrm{pg} / \mathrm{mL}$ & $5 \mathrm{ng} / \mathrm{mL}$ \\
\hline
\end{tabular}




\subsection{Experimental}

Experimental sections 4.2.1 \& 4.2.2 describe method development steps common to all three UPLC-MS/MS methodologies. These sections are designed to provide the reader with an overview of the steps taken and processes followed during preliminary method development. Pease refer to individual experimental sections in chapters 5, 6 and 7 for specific instrumental conditions and detailed experimental designs. Section 4.3 describes the details of method validation experiments for each of the three UPLC-MS/MS methodologies. Please refer to chapter 3 for definitions of validation parameters and generalized experimental approaches. Please refer to the individual results and discussion sections in chapters 5, 6, and 7 for discussion and interpretation of individual validation experiments.

\subsubsection{Development and Optimization of MS/MS Parameters}

Mass spectrometric detection was performed using a Waters TQD triple quadrupole mass spectrometer (Waters Corp., Milford, MA, USA) equipped with an electrospray ionization (ESI) source operating in positive ion mode. Method development was initiated by identifying appropriate quantifier and qualifier mass transitions for each analyte. Concentrated $(10 \mu \mathrm{g} / \mathrm{mL})$ solutions each analyte were directly infused into the mass spectrometer ionization source at a flow rate of $20 \mu \mathrm{L} / \mathrm{min}$. The flow paths of the concentrated analyte solutions were modified with a T-mixer to allow mixing of the solution with mobile phase (Figure 14). This allowed for the simultaneous infusion of analyte solution and initial mobile phase into the mass spectrometer. Simultaneous infusion of the sample with the initial mobile phase ensured that any subsequently optimized tune page parameters were compatible with the initial mobile phase. Optimizing the tune page parameters under these conditions also ensured that analytes were being introduced 
into the ESI source in an environment that was representative of an authentic sample. This ensured the most accurate optimization of ionization parameters specific to both the sample and the mobile phase.

Figure 14: Schematic representation of the 'T-mixing' mode which allows for the simultaneous infusion of analyte solution from reservoir A and mobile phase eluting from the UPLC column.

Concentrated $(10 \mu \mathrm{g} / \mathrm{mL})$ solutions of each analyte were concomitantly infused with the initial mobile phase composition at a flow rate of $0.6 \mathrm{~mL} / \mathrm{min}$. During infusion of each analyte, the collision gas was turned off to allow the protonated molecular ion of each compound to reach the detector and produce a recordable signal. Following identification of the molecular ion signal, an auto tune was completed for each analyte which involved adjusting the capillary voltages, cone voltages, and collision energies to maximize the signal for both the precursor ions and the product ions generated in the collision cell. Auto tuning of the protonated molecular ion of each compound yielded information necessary to collect data in the MRM mode. The mass transition 
from the protonated molecular ion $[\mathrm{M}+\mathrm{H}]^{+}$to the most abundant product ion was designated the quantifying ion transition while the second most abundant mass transition was designated as the qualifying ion transition for each analyte. The most abundant product ion for each deuterated internal standard was also monitored and used to calculate the response ratio between internal standards and analytes for all experiments. Following auto tuning of each analyte and internal standard, the optimized parameters were used to construct the MS/MS method which was then used to acquire data in the MRM mode.

\subsubsection{Development of UPLC Separation Conditions}

Following development of the MS/MS detection method, analytes were individually injected onto the UPLC to optimize chromatographic separation conditions. Analytes were individually injected onto the column to obtain and record characteristic retention times and to optimize chromatographic conditions. Chromatographic conditions were optimized to ensure that all peak shapes were Gaussian in nature and baseline resolution was achieved, allowing for accurate and reliable identification. All liquid chromatographic separations were performed on a Waters ACQUITY ${ }^{\mathrm{TM}}$ ultra performance liquid chromatograph (UPLC ${ }^{\mathrm{TM}}$ ) (Waters Corp., Milford, MA, USA). Separations were achieved on one of several ACQUITY UPLCTM columns (see individual experimental sections for column dimensions). All mobile phases consisted of deionized water containing $0.1 \%$ formic acid (solvent $\mathrm{A}$ ), and acetonitrile containing $0.1 \%$ formic acid (solvent B). Analytes elution was achieved using step-wise binary elution gradients and all flow was directed into the ESI source of the mass spectrometer.

\subsubsection{Considerations for UPLC-MS/MS Method Development}

Analytical separation and detection of xenobiotics in complex biological matrices can prove difficult when employing detection systems that lack the selectivity of mass spectrometry. 
Traditional techniques such as HPLC equipped with UV detection require baseline separation of analytes from any endogenous interferences, xenobiotics, or sample contaminants with similar UV absorbance. UV detection systems measure the UV absorbance of column eluates at a particular wavelength or over a range of wavelengths. Compounds with similar chromatographic properties to the analyte of interest, which absorb UV radiation at the chosen wavelength may co-elute with the analyte making any subsequent attempts at integration and quantification inaccurate and unreliable. The problem of co-elution can be overcome by modifying sample extraction techniques, reconstitution solvents, mobile phase components, flow rates, chromatographic columns or detection wavelengths.

Depending on the complexity of the biological extract, lengthy, complex chromatographic separations employing various mobile phase additives may be required to successfully resolve the analyte of interest from sample interferences. Mass spectrometry, in particular tandem mass spectrometry, offers the analyst enhances selectivity for the identification of low levels of analyte in complex biological matrices rich in interferences. According to the SOFT/AAFS forensic laboratory guidelines, the detection or initial identification of drugs or toxins should be confirmed whenever possible by a second testing technique based on a different chemical principle. Due to its superior sensitivity, mass spectrometry is the recommended confirmatory technique, where possible and practical[119].

Confirmation assays in the forensic toxicology laboratory which employ mass spectrometry are generally performed in the single ion monitoring (SIM) mode for MS analysis and in the multiple reaction monitoring (MRM) mode MS/MS analysis. This is the general occurrence due to the sequence of testing procedures performed in the routine toxicological analysis of blood, urine, or alternate specimens. Confirmation techniques employing mass 
spectrometry are generally developed and optimized to identify individual components of a drug class whose presence was indicated by the presumptive screen test.

Due to the finite number of analytes monitored during a confirmation assay, data acquisition can be performed in either the SIM mode for single mass spectrometry experiments or MRM for tandem mass spectrometry techniques. SIM and MRM scanning during confirmation analysis provides the analyst with inherent selectivity for the analyte/s of interest and due to the reduction in background signal, sensitivity is also enhanced. Tandem mass spectrometry experiments employing the MRM mode do not produce mass spectrums, rather chromatograms as the data output is reflective of only one mass transition corresponding to the analyte of interest rather than a complete mass spectrum obtained by scanning a certain mass range. While chromatographic separations still play an integral role in LC-MS/MS assays, baseline chromatographic resolution is often not required when employing mass spectrometry as a detection technique because of its ability to monitor mass transitions specific to an individual analyte. When operating in MRM mode, mass spectrometers are constantly acquiring data for one or more mass transitions. Although co-elution may exist between transitions on the total ion chromatogram (TIC), data pertaining to each transition is being acquired individually and extraction of the individual trace allow for the accurate and reliable quantification of the analyte corresponding to that transition. Figure 15 shows the TIC for the chromatographic separation of eight tricyclic antidepressants on a Waters Acquity TQD. It can be seen from the TIC, which only contains five peaks, that the individual analytes are not chromatographically resolved. When operating in MRM mode however, triple quadrupole instruments are constantly acquiring data for individual ion traces. This information can be extracted from the TIC and used to quantify each analyte. Figure 16 shows the extracted ion traces for each analyte. While 
chromatographic resolution was not achieved, all eight analytes can be accurately quantified using individual MRM ion traces (Figure 16).

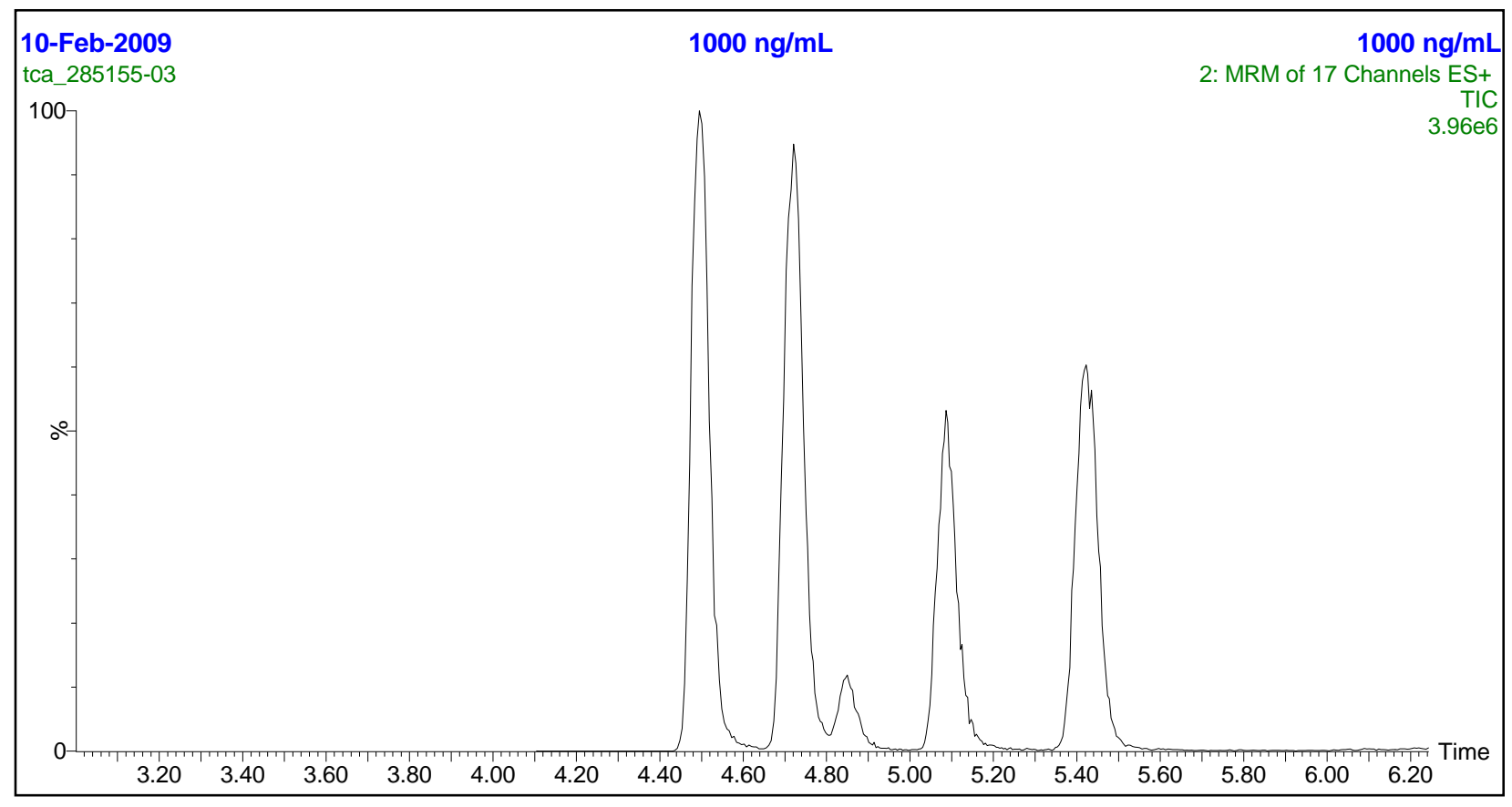

Figure 15: TIC for the chromatographic separation of eight tricyclic antidepressants on a Waters Acquity TQD. Analytes are not chromatographically resolved but can be quantified using their individual MRM ion traces. 


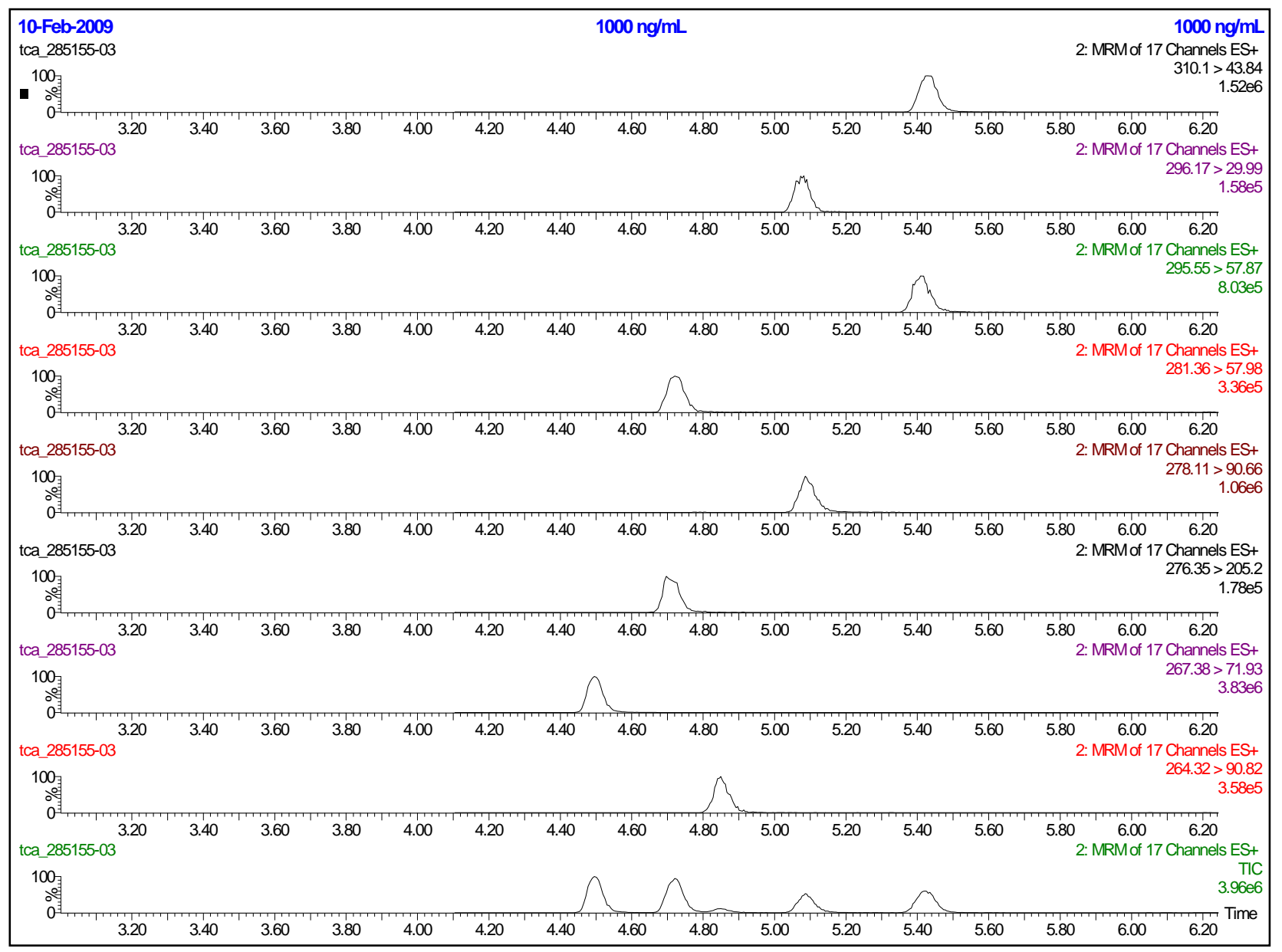

Figure 16: Extracted in traces for each analyte. Individual ion traces are acquired in the MRM mode and eliminate the need for chromatographic resolution.

There are however, circumstances in which baseline resolution is required for tandem mass spectrometric detection to enable accurate and reliable quantitative results. One such instance arises when the detection of two or more co-eluting compounds is performed by monitoring the abundance of product ions with identical masses. Chromatographic resolution is required in such cases to eliminate the detrimental effects of 'cross-talk' which is caused by the slow removal of ions from the collision cell. Cross-talk may occur when two co-eluting compounds with identical product ion masses are monitored by successive transition scans[196]. Cross-talk occurs when fragment ions from a certain mass transition scan event have not fully 
cleared the collision cell before a second scan event takes place in which the same fragment ion is monitored. Residual fragment ions from the first scan event contributing to the analyte response for the second scan event lead to signal artifacts which impact the quantitation of the co-eluting analytes.

The effects of cross talk can be minimized by introducing an inter-scan delay between the two transitions to ensure optimal clearance of the collision cell, however this increases the method 'dead time' and significantly affects the scan cycle time. Increasing the scan cycle time by introducing an inter-scan delay, decreases the number of data points obtained over a chromatographic peak which can also have a detrimental effect of quantitation accuracy[196]. In such cases, chromatographic resolution is the preferred option for alleviating the problems associated with ion cross-talk. Chromatographic resolution may not always be feasible for methods designed to detect a large number of analytes in a relatively short chromatographic run[196, 197] and in such cases, the effects of cross-talk on quantitation accuracy should be assessed during validation experiments.

\subsection{Experimental Design for Method Validation}

Preliminary method development experiments described in section 4.2 involved developing and optimizing a chromatographic separation which allowed for the accurate and reliable identification of each analyte and deuterated internal standard based on retention times. Experiments also involved developing and optimizing a mass spectral method capable of obtaining data in the multiple reaction monitoring (MRM) mode for each analyte and deuterated internal standard. Following the development and optimization of UPLC and MS/MS conditions using concentrated drug standards, each UPLC-MS/MS methodology was subject to a full 
analytical method validation. Validation experiments were designed and carried out to determine the suitability of each method for its intended purpose.

Scientific validation of the UPLC-MS/MS methodologies included performance studies assessing matrix equivalence, linearity, selectivity, accuracy, intra- and inter-run precision, recovery, stability and limit of detection (LOD). Method validation studies were performed over a ten day time period and methodologies were deemed suitable for the analysis of authentic samples if, and only if, validation studies had were completed and acceptance criteria were met for each validation parameter. Several validation experiments required the replicate analysis of standards prepared at concentrations spanning the concentration range for that specific analyte. With the exception of matrix equivalence experiments (see section 4.3.1) standards were prepared at the LLOQ, the ULOQ, and a concentration midway between the two. Table 8 lists the concentrations of all standards used during validation experiments. 
Table 8: Concentrations of standards used in all validation experiments. Concentrations of standards spanned the calibration range of each method.

\begin{tabular}{|c|l|c|c|c|}
\hline Method & \multicolumn{1}{|c|}{ Analyte } & $\begin{array}{c}\text { Conc of Std. 1 } \\
\text { (LLOQ) }\end{array}$ & $\begin{array}{c}\text { Conc of Std. 2 } \\
\text { (midway point) }\end{array}$ & $\begin{array}{c}\text { Conc of Std. 3 } \\
\text { (ULOQ) }\end{array}$ \\
\hline $\begin{array}{c}\text { Methodology } \# 1 \\
\text { Chapter 5 }\end{array}$ & Cocaine & $10 \mathrm{ng} / \mathrm{mL}$ & $50 \mathrm{ng} / \mathrm{mL}$ & $250 \mathrm{ng} / \mathrm{mL}$ \\
\cline { 2 - 5 } & Benzoylecgonine & $10 \mathrm{ng} / \mathrm{mL}$ & $50 \mathrm{ng} / \mathrm{mL}$ & $250 \mathrm{ng} / \mathrm{mL}$ \\
\hline \multirow{3}{*}{$\begin{array}{c}\text { Methodology } \# 2 \\
\text { Chapter 6 }\end{array}$} & Codeine & $10 \mathrm{ng} / \mathrm{mL}$ & $50 \mathrm{ng} / \mathrm{mL}$ & $500 \mathrm{ng} / \mathrm{mL}$ \\
\cline { 2 - 5 } & Hydromorphone & $10 \mathrm{ng} / \mathrm{mL}$ & $50 \mathrm{ng} / \mathrm{mL}$ & $500 \mathrm{ng} / \mathrm{mL}$ \\
\cline { 2 - 5 } & 6-monoacetylmorphine & $10 \mathrm{ng} / \mathrm{mL}$ & $50 \mathrm{ng} / \mathrm{mL}$ & $500 \mathrm{ng} / \mathrm{mL}$ \\
& & $10 \mathrm{ng} / \mathrm{mL}$ & $50 \mathrm{ng} / \mathrm{mL}$ & $500 \mathrm{ng} / \mathrm{mL}$ \\
\hline \multirow{2}{*}{$\begin{array}{c}\text { Methodology } \# 3 \\
\text { Chapter 7 }\end{array}$} & Amphetamine & $5 \mathrm{ng} / \mathrm{mL}$ & $50 \mathrm{ng} / \mathrm{mL}$ & $500 \mathrm{ng} / \mathrm{mL}$ \\
\cline { 2 - 5 } & Methamphetamine & $5 \mathrm{ng} / \mathrm{mL}$ & $50 \mathrm{ng} / \mathrm{mL}$ & $500 \mathrm{ng} / \mathrm{mL}$ \\
\hline
\end{tabular}

\subsubsection{Equivalence Studies for Calibration Curves}

Certified drug free meconium cannot be purchased for the purpose of constructing calibration curves. In order to alleviate this problem, initial equivalence studies were performed to determine the suitability of using calibration curves constructed in blood for the quantitation of meconium specimens.

Experiments were performed by quantitating five high quality control (HQC) standards and five low quality control (LQC) standards using a calibration curve constructed in blood and a calibration curve constructed in meconium which had previously screened negative at AIT Laboratories employing a cutoff of $50 \mathrm{ng} / \mathrm{g}$. Equivalence studies were performed to ensure that calibration curves prepared in blood were able to accurately quantify quality control standards, 
and therefore authentic samples, in meconium. Quantitative values obtained for QC standards using the calibration curve constructed in blood were required to be within $15 \%$ relative error $(\% \mathrm{RE})$ of the quantitative values obtained using the calibration curve constructed in meconium. If equivalence studies indicated that calibration curves constructed in blood were capable of accurately quantitating QC standards prepared in meconium, certified drug free whole blood would be employed for the remainder of validation for the purpose of constructing calibration curves.

Preliminary equivalence studies were performed on the first day of validation to assess the suitability of calibration curves constructed in certified drug-free blood for the purpose of quantitating meconium specimens. High and low quality control specimens were prepared according the calibration range of each assay. The concentration of standards used in matrix equivalence studies varies slightly from those used in the remainder of validation experiments. Table 9 lists the concentration of all standards used for equivalence studies.

Table 9: Concentrations of all LQC and HQC standards used in preliminary matrix equivalence studies.

\begin{tabular}{|l|c|c|}
\hline \multicolumn{1}{|c|}{ Analyte } & LQC used for equivalence studies & HQC used for equivalence studies \\
\hline Cocaine & $15 \mathrm{ng} / \mathrm{mL}$ & $125 \mathrm{ng} / \mathrm{mL}$ \\
\hline Benzoylecgonine & $15 \mathrm{ng} / \mathrm{mL}$ & $125 \mathrm{ng} / \mathrm{mL}$ \\
\hline Morphine & $10 \mathrm{ng} / \mathrm{mL}$ & $500 \mathrm{ng} / \mathrm{mL}$ \\
\hline Codeine & $10 \mathrm{ng} / \mathrm{mL}$ & $500 \mathrm{ng} / \mathrm{mL}$ \\
\hline Hydromorphone & $10 \mathrm{ng} / \mathrm{mL}$ & $500 \mathrm{ng} / \mathrm{mL}$ \\
\hline 6-monoacetylmorphine & $10 \mathrm{ng} / \mathrm{mL}$ & $500 \mathrm{ng} / \mathrm{mL}$ \\
\hline Amphetamine & $10 \mathrm{ng} / \mathrm{mL}$ & $500 \mathrm{ng} / \mathrm{mL}$ \\
\hline Methamphetamine & $10 \mathrm{ng} / \mathrm{mL}$ & $500 \mathrm{ng} / \mathrm{mL}$ \\
\hline
\end{tabular}

Quantitative values obtained for QC standards using a calibration curve constructed in blood were required to be within $15 \%$ relative error $(\% \mathrm{RE})$ of the quantitative values obtained using a 
calibration curve constructed in negative meconium. Relative error was calculated according to Equation 13.

$\% \mathrm{RE}=[($ value from blood curve/value from meconium curve $)-1] \times 100 \% \quad$ Equation 13

\subsubsection{Selectivity}

The selectivity of each LC-MS/MS assay was assessed by analyzing five blank meconium samples which had previously screened negative at AIT Laboratories using a cutoff of $50 \mathrm{ng} / \mathrm{g}$. Negative meconium samples were analyzed as blanks because certified drug free meconium is not commercially available. Blank samples $(n=5)$ were analyzed using each of the three confirmation methods employing appropriate internal standards, calibration curves and quality control standards to ensure that the method would be selective for the analytes of interest in the presence of any endogenous interferences. Methods were deemed selective if, and only if, the blank matrices did not generate an analyte response in excess of the LLOQ (see Table 8 for method LLOQs). Following the analysis of blank sample matrices for the presence of possible interferences, selectivity was established at the LLOQ for each analyte. In order to deem the methodology selective, negative meconium specimens were spiked with the analyte/s of interest at a concentration corresponding to the LLOQ. Specimens were then spiked with varying concentrations of potential interfering species to ensure that the method was selective for the analyte/s of interest in the presence of other endogenous matrix components. Samples were again analyzed by each of the confirmation methods using appropriate internal standards, calibration curves and quality control standards. Individual methodologies were deemed selective if, and only if, they were capable of quantifying the analyte/s of interest within $+/-20 \%$ of the expected nominal value for $80 \%$ of the prepared samples. 


\subsubsection{Accuracy}

Accuracy of the analytical methodology was assessed by preparing and analyzing five replicate samples prepared in negative meconium at concentrations covering the calibration range of the method. Samples prepared at the LLOQ, and ULOQ and a concentration midway between the two were analyzed to assess the accuracy of the quantitation. The method was deemed accurate if, and only if, the values obtained for each specimen were within $15 \%$ of the expected nominal values, except at the LLOQ where values were expected to be within $20 \%$ of the expected nominal value. Table 8 lists the concentration of all standards used for accuracy experiments.

\subsubsection{Precision}

The precision of each confirmation assay was assessed by analyzing five replicates at three different analyte concentrations corresponding to the LLOQ, the ULOQ and a concentration point midway between the LLOQ and the ULOQ. Methodologies were deemed precise if, and only if, the relative standard deviation (RSD) did not exceed $15 \%$, except for the LLOQ, where the RSD was required to be less than $20 \%$. Intra batch precision was assessed by analyzing five replicates at three different concentrations within a single analytical run and inter batch precision was assessed by analyzing replicates over a four day/four batch period. Inter batch precision was assessed to determine the methods precision with time, and to provide an indication of method robustness. Intra batch precision was calculated by determining the standard deviation $(\sigma)$ and the mean $(\mu)$ for each the five replicate analyses at each concentration. The absolute value of the coefficient of variation or the relative standard deviation (RSD) was then calculated according to Equation 14. 
Inter batch precision was calculated in the same manner using standard deviation $(\sigma)$ and mean ( $\mu$ ) values calculated from values obtained over the four day period for each concentration.

\subsubsection{Recovery}

Recovery was evaluated to determine extraction efficiencies of each analyte from the meconium matrix. Recovery was determined by directly comparing the detector response (mean peak area) for extracted samples prepared and analyzed in triplicate at concentrations corresponding to the LLOQ, the ULOQ and a concentration midway between the two, with detector responses for unextracted samples at the same concentrations which represented $100 \%$ recovery[199]. Unextracted samples were prepared directly in mobile phase and injected.

Recoveries were determined as a percentage (Equation 15) and although a high percent recovery was not necessary for validation, percent recoveries were required to be consistent and reproducible within triplicate samples and over the three concentrations studied.

$\%$ Recovery $=($ response for extracted $) /($ response for unextracted $) \times 100 \% \quad$ Equation 15

\subsubsection{Limit of Detection (LOD) and Limit of Quantitation (LOQ)}

Limit of detection studies were performed by analyzing serially dilute standards of each analyte until retention time shifts or inaccurate ion ratios prevented accurate and unequivocal identification. A series of blank meconium specimens were spiked with each analyte at serially dilute concentrations beginning with the LLOQ. Samples were analyzed by the appropriate confirmation method using internal standards, calibration curves and quality control standards. The LOD for each methodology was determined by evaluating analyte responses, retention time 
accuracy, and ion ratio accuracy for each analyte at each concentration. The limit of detection was identified as the lowest concentration of analyte which could be accurately identified but not necessarily quantified. The increased sensitivity of the UPLC-MS/MS methodology meant that analyte responses were observed at concentrations lower than the LOD, however, retention time shifts and inaccurate ion ratios meant that unequivocal identifications could not be made. The limit of detection was identified as the lowest concentration of analyte which could be identified using established retention time windows and ion ratio abundances.

In order for a methodology to be considered accurate and precise, quantifications at the LLOQ are generally required to exhibit precision within $20 \%$ of the coefficient of variation (CV) RSD and accuracy within $+/-20 \%$ of the expected nominal concentration [198]. The lowest point of each calibration curve was subject to these exact criteria for acceptance during all validation experiments and as a result, the LLOQ was subsequently defined as the concentration of the lowest calibration standard for each confirmation assay.

\subsubsection{Stability}

In order to assess the stability of each analyte in meconium, freshly made stock solutions of each analyte were prepared and used to spike blank meconium specimens which were then subject to several freeze and thaw cycles and subsequently analyzed. Blank meconium specimens which had previously screened negative at AIT Laboratories using a cutoff of $50 \mathrm{ng} / \mathrm{g}$ were spiked with analyte at concentrations corresponding to the LLOQ and the ULOQ (Table 8). Specimens were then frozen for 24 and subsequently thawed unassisted at room temperature. Once thawed, the specimens were again frozen under the same conditions for another 24 hours after which they were thawed again at room temperature. This cycle was completed a total of three times and specimens were analyzed upon thawing on the third freeze thaw cycle. Each 
analyte was deemed stable during storage if, and only if, the quantitative values obtained were within $20 \%$ of the expected nominal concentration.

\subsubsection{Data Analysis}

Analytical data was analyzed using Masslynx version 4.1 software. Criteria for a positive result included accurate chromatographic retention time, presence of both the qualifying product ion and the quantifying product ion, and product ion ratios within acceptable limits[119, 199]. Chromatographic retention times are initially established for each analyte and deuterated internal standard during method validation through the analysis of reference standards. Retention times are updated following the analysis of calibration standards in each batch to account for minor drifts in daily retention times. Retention times for each analyte of interest were required to be within $5 \%$ of those determined with control samples. The ratio of the quantifying product ion peak area to the qualifying product ion peak area was required to be within $+/-20 \%$ of the ion ratio determined for calibrators. Quantitation was performed using a working standard calibration curve and comparing the ratio of quantifying ion peak area to internal standard peak area. Authentic samples were required to exhibit sufficient recovery of the internal standard. Internal standard response was required to be between $10 \%$ and $200 \%$ of the calibrator/control average. Calibration curves were required to comprise at least $50 \%$ of the original curve points and any specimens with quantitative values above greater than the upper calibrator were required to be rerun at an appropriate dilution. The analytical run is considered acceptable if the calculated concentrations of analyte/s in control samples are within $20 \%$ of the expected nominal value. 


\section{Chapter 5: Detection and Quantification of Cocaine and Benzoylecgonine in Meconium Using Solid Phase Extraction and UPLC-MS/MS}

\subsection{Abstract}

A methodology for the selective determination and quantification of cocaine and its major metabolite benzoylecgonine in meconium using ultra performance liquid chromatographytandem mass spectrometry (UPLC-MS/MS) is described. Past studies indicate that up to $40 \%$ of neonates dying within two days of birth with no apparent cause of death have cocaine and/or benzoylecgonine in their blood, and rates of infants exposed to cocaine prenatally has been estimated to be between $2.6 \%$ and $11 \%$ of all live births. Ultra performance liquid chromatography (UPLC) is an emerging analytical technique which draws upon the principles of chromatography to run separations at higher flow rates for increased speed, while simultaneously achieving superior resolution and sensitivity. Extraction of both analytes was achieved using a preliminary protein precipitation followed by solid-phase extraction (SPE). Limits of detection for both analytes were $4 \mathrm{ng} / \mathrm{g}$ and the lower limit of quantitation (LLOQ) was $40 \mathrm{ng} / \mathrm{g}$. The working calibration range was $40-1000 \mathrm{ng} / \mathrm{g}$. The methodology exhibited high intra-run precision with $\mathrm{CV}$ values ranging from $1.6-9.2 \%$ for cocaine and 5.3-11.8\% for benzoylecgonine. Inter run precision was evaluated and experiments produced $\mathrm{CV}$ values ranging from $3.9-5.0 \%$ for cocaine and 4.4-6.2\% for benzoylecgonine. The increased speed and separation efficiency offered by UPLC, allowed for the separation and subsequent quantification of both analytes in less than 2 minutes. Dramatic increases in separation speed such as those afforded by UPLC translate into increased samples per unit time in high throughput toxicology laboratories. Development of sensitive analytical methodologies capable of detecting low levels of such drugs in meconium will prove beneficial for the identification of prenatal substance abuse. 


\subsection{Introduction}

Cocaine belongs to the tropane alkaloid family and is obtained from the leaves of the plant Erythroxylon coca. Although the leaves of Erythroxylon coca and other related species have been used by the Peruvian Indians for centuries to increase endurance and improve wellbeing, the active ingredient cocaine, was not isolated until the mid nineteenth century. Albert Niemann, a graduate student at the University of Gottingen was the first person to devise and report a technique for the isolation of cocaine in 1860[204]. In 1884, 24 years after the first reported isolation of cocaine, Dr Karl Koller discovered that cocaine was an effective local anesthetic. In the years following Koller's discovery, physicians around the world were employing cocaine as an anesthetic in ophthalmologic, dental, and general surgical procedures. Although analogues of cocaine such as procaine and lidocaine are still employed as anesthetic agents, the free distribution of cocaine was banned by the Harrison Narcotics Act in 1914. By the mid twentieth century the recreational use of cocaine had become a significant concern across socioeconomic lines.

Cocaine is available on the street in either the base form (crack, free base) or as the hydrochloride salt. Although both forms of the drug are available in high purity for a similar street value, the free-base 'crack' cocaine is predominately used for smoking while cocaine hydrochloride is mainly employed for intravenous injection and nasal insufflation.

The prevalence of cocaine use has increased substantially over the past two decades and in 1989 it was estimated that 50 million Americans has used cocaine at least once and approximately 8 million people were regular users of the drug[205]. More recent findings from the 2006 National survey on drug use and health (NSDUH) estimates 2,700 initiates to cocaine use per day based on the 977,000 persons aged 12 years or older who admitted to using cocaine 
for the first time within the past twelve months[2]. The NSDUH estimates that in addition to the 2.4 million frequent (i.e., at least twice weekly) users of cocaine, there are approximately 4.6 million occasional users (i.e., once a month or less) of the drug, excluding individuals already in prison[2].

Although it is difficult to accurately determine the number of women who use cocaine during pregnancy, there is evidence to suggest that cocaine represents a significant proportion of the illicit substances which $4 \%$ of pregnant women admitted using during pregnancy in the 2006 NSDUH $[2,8,11,206]$. One such study, performed at a high-risk tertiary perinatal, reported that $38 \%$ of infants tested positive for drugs, and $90 \%$ of those 773 infants were positive for cocaine[207]. In 1991 the Los Angeles County Office of Chief Medical Examiner-Coroner (LAC CMEC) reported that $40 \%$ of neonates dying within two days with no apparent cause of death had cocaine and/or benzoylecgonine in their blood[208]. Rates of infants exposed to cocaine prenatally has been estimated to be between $2.6 \%$ and $11 \%$ of all live births[11].

Chemical extraction of benzoylecgonine from biological matrices is complicated by the amphoteric nature of the compound. While cocaine exists as a basic molecule and is easily extracted into organic solvent under alkaline conditions, benzoylecgonine exists as a zwitterion making it difficult to extract. Extraction of target analytes from biological matrices generally occurs following neutralization with an appropriate acid or base depending on the properties of the drug. Benzoylecgonine possesses two ionizable centers, one with acidic properties and one with basic properties making its neutralization difficult. Extraction design is further complicated by the need to simultaneously extract cocaine for analysis meaning that the method of choice must provide satisfactory recovery of both analytes. 
Table 10 provides the chemical structures of cocaine and its primary metabolite, benzoylecgonine and describes certain physicochemical properties of each compound including molecular weights, molecular formulas, and pKa's.

Table 10: Chemical structures, molecular weights, molecular formulas, and pKa's of cocaine and benzoylecgonine.

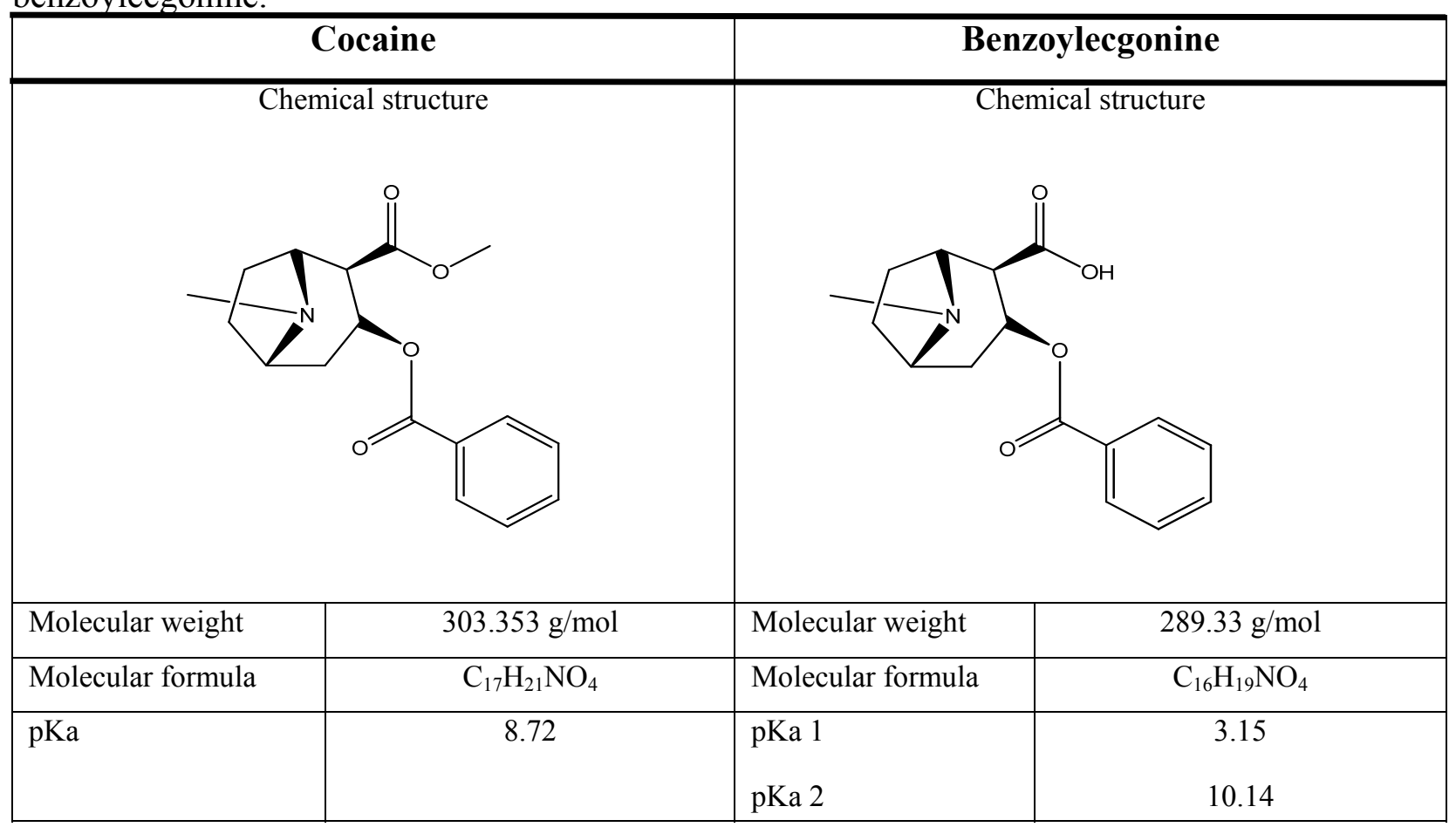

\subsection{Mechanism of Action}

Cocaine is a naturally-occurring central nervous system (CNS) stimulant that interferes with the actions of dopamine, norepinephrine and serotonin in functioning nerves. Cocaine is classed as a sympathomimetic agent due to its ability to activate the sympathetic nervous system both centrally and peripherally[209]. Central stimulation of the sympathetic nervous system results from cocaine's ability to selectively bind dopamine reuptake transporters (DATs) in the brain[204]. Clearance of dopamine at the synapse and subsequent termination of dopaminergic neurotransmission is achieved through reuptake into the presynaptic neuron which is mediated by DATs[210]. By binding to DATs, cocaine impairs the reuptake of dopamine into the 
presynaptic neuron resulting in elevated dopamine levels in the central nervous system. Direct stimulation of the central nervous system by cocaine and other sympathomimetic agents also results in increased norepinephrine release from peripheral synapses. Cocaine not only facilitates increased release of norepinephrine peripherally it also acts to inhibit its reuptake at the synapses causing it to remain in the synaptic cleft for a prolonged period of time. As a result of cocaine's ability to inhibit the reuptake of dopamine centrally and norepinephrine peripherally, the natural effect of these neurotransmitters is amplified. Excessive levels of CNS dopamine and peripheral norepinephrine account for the feelings of euphoria and increased alertness associated with cocaine use[209].

\subsection{Effect of Prenatal Exposure}

Due to the popularity and prevalence of cocaine use in the USA, the vast majority of research conducted into the effects of maternal drug use has focused on cocaine. Prenatal cocaine use has been associated with premature labor, placental abruption, low birth parameters (weight, head circumference, length), microcephaly, congenital malformations, increased risk of sudden infant death syndrome (SIDS), spontaneous abortion, acute hypoxic-ischemic encephalopathy, cerebral hemorrhage or infarction, abnormal neonatal behavior, limb deformities[75, 83, 211, 212].

\subsection{History of Cocaine and Benzoylecgonine Detection in Meconium Specimens.}

The detrimental outcomes of prenatal cocaine exposure on developing children have long been realized. As a result of the widespread use of cocaine during pregnancy, many analytical methodologies describing the detection of cocaine and its inactive metabolite, benzoylecgonine, in meconium have appeared in the literature. 
Browne and colleagues described a method for the detection of cocaine and its metabolites in meconium using HPLC and GC-MS. First-day meconium samples were subject to solid phase extraction followed by chromatographic separation and detection by either UV or MS. Although the authors reported the presence cocaine at concentrations ranging from $0.1 \mu \mathrm{g} / \mathrm{g}$ to $0.78 \mu \mathrm{g} / \mathrm{g}$, no benzoylecgonine, ecgonine or ecgonine methyl ester was detected, prompting the authors to speculate that the metabolizing capabilities of the neonate may be limited[52]. Callahan and co-workers employed GC/MS for the identification of gestational cocaine exposure in the infants of 59 women who had been previously identified as cocaine users through hair analysis. The authors concluded that GC/MS analysis of meconium proved more sensitive than immunoassay analysis of urine and can detect fetal cocaine exposure which occurred in the last two trimesters of pregnancy[213].

Clark et al employed solid phase extraction and GC/MS for the determination of cocaine and benzoylecgonine in methanolic extracts of meconium which had been screened using fluorescence polarization immunoassay (FPIA). The enhanced sensitivity of GC/MS enabled the authors to identify cocaine at concentrations as low as $0.25 \mu \mathrm{g} / \mathrm{g}$ and benzoylecgonine at concentrations of $0.5 \mu \mathrm{g} / \mathrm{g}[214]$. Murphey and co-workers employed solid phase extraction columns with both cation exchange and hydrophobic properties for the simultaneous extraction of cocaine, benzoylnorecgonine, benzoylecgonine and norcocaine from meconium. Following extraction, analytes were identified using HPLC and limits of detection were 50ng/g for all analytes[215].

Many of the reported methodologies for the determination of cocaine and its metabolites in meconium utilized solid phase extraction followed by GC/MS analysis. Solid phase extraction is generally required due to the complex nature of the meconium matrix. Liquid-liquid 
extractions fail to remove many of the endogenous interferants present in the sample. GC/MS analysis was routinely utilized following SPE throughout the 1990s due to the separating power of gas chromatography and the unequivocal mass spectrometric identifications afforded my MS. Early reports of LC-MS/MS methods for the determination of cocaine and its metabolites in meconium began to appear in the literature at the turn of the century. Xia and colleagues employed a triple quadrupole mass spectrometer equipped with an electrospray ionization source for the determination of cocaine and several of its metabolites following LC separation. The authors conclude that the utilization of MS-MS both increases the selectivity of the assay and reduces sample preparation time as several metabolites of cocaine which are directly amendable to LC separation require additional derivatization for GC analysis[216]. Pichini and co-workers employed an ESI-LC/MS assay operating in SIM mode for the simultaneous determination of cocaine and opiates in meconium. Analytes were extracted using an initial liquid extraction into methanol or ammonium hydrogen carbonate buffer followed by a solid phase extraction for subsequent sample clean-up. Method validation included a linear dynamic range of $0.005-$ $1.00 \mu \mathrm{g} / \mathrm{g}[217]$. The same authors re-applied the validated LC-MS method two years later for the determination of two additional cocaine metabolites in meconium[218].

To the best of the author's knowledge, this is the first report of the application of UPLCMS/MS for the determination of cocaine and benzoylecgonine in meconium.

Validation and implementation of UPLC-MS/MS methodologies capable of detecting low levels of common drugs of abuse in meconium will assist in providing more accurate information pertaining to the prevalence of prenatal drug exposure. The increased separation efficiency of UPLC provides the analyst with maximal separation power necessary to resolve endogenous sample components from target analytes. Increases in sensitivity, afforded by taller, 
narrower peaks are also achieved with UPLC separations. As a result, low levels of target analyte, which would likely go undetected using traditional HPLC, will be detectable in the complex meconium matrix. The introduction of tandem mass spectrometry detection into routine meconium analysis provides obvious advantages such as enhanced selectivity relative to UV or single mass spectrometric detection. Increases in selectivity ensure that low concentrations of target analytes, which would normally be indistinguishable from endogenous interferences using HLPC-UV or GC/MS, are accurately identified.

\subsection{Experimental}

\subsubsection{Chemicals and Reagents}

Cocaine, benzoylecgonine, cocaine- $\mathrm{d}_{3}$, and benzoylecgonine- $\mathrm{d}_{3}$ standards $(1 \mathrm{mg} / \mathrm{mL}$ in methanol) were obtained from Cerilliant (Round Rock, TX). ISOLUTE ${ }^{\circledR}$ HM-N supported liquid-liquid extraction columns were purchased from Biotage (Charlottesville, VA). All solvents were HPLC grade and obtained from Fisher Scientific (Pittsburgh PA).

\subsubsection{Calibration Curve Matrix}

Meconium specimens which had previously screened negative for cocaine using a 50 $\mathrm{ng} / \mathrm{g}$ cutoff at AIT laboratories (Indianapolis, IN) were collected and spiked with both cocaine and benzoylecgonine to give concentrations of $15 \mathrm{ng} / \mathrm{mL}(\mathrm{n}=5)$ and $125 \mathrm{ng} / \mathrm{mL}(\mathrm{n}=5)$. Spiked meconium was then quantified using a calibration curve constructed in negative meconium and a calibration curve constructed in certified drug free whole blood. Quantitative results obtained using the meconium calibration curve showed excellent correlation $(<15 \% \mathrm{CV})$ with those obtained using the calibration curve made up in negative blood and as a result, all subsequent 
method validation experiments were performed using calibration curves prepared in certified negative blood.

\subsubsection{Working Standards}

Calibration curves were constructed using seven calibration standards prepared in certified drug free whole blood and all sample volumes were $1 \mathrm{~mL}$. A working standard (500 $\mathrm{ng} / \mathrm{mL}$ ) used to prepare calibration standards, was prepared by combining $250 \mu \mathrm{L}$ of a stock solution $(10 \mu \mathrm{g} / \mathrm{mL})$ of cocaine and benzoylecgonine with $4.75 \mathrm{~mL}$ of deionized water. An internal standard solution $(500 \mathrm{ng} / \mathrm{mL})$ was prepared using $1 \mathrm{mg} / \mathrm{mL}$ standards of cocaine- $\mathrm{d}_{3}$ and benzoylecgonine- $\mathrm{d}_{3}$.

\subsubsection{Calibration Curves}

Calibration curves for all experiments were prepared according to Table 11.

Table 11: Preparation of cocaine and benzoylecgonine calibration curves.

\begin{tabular}{|c|c|c|}
\hline Standard Concentration & Volume of Working Standard & Volume of Deionized water \\
\hline 250 & 500 & 500 \\
\hline 100 & 200 & 800 \\
\hline 50 & 100 & 900 \\
\hline 25 & 50 & 950 \\
\hline 10 & 20 & 980 \\
\hline 5 & 10 & 990 \\
\hline Negative & 0 & 1000 \\
\hline
\end{tabular}

$100 \mu \mathrm{L}$ of internal standard solution $(500 \mathrm{ng} / \mathrm{mL})$ was added to each sample including the blank and all samples were then vortexed for 30 seconds.

\subsubsection{Quality Control (QC) Standards}

Quality control standards (QC's) were prepared and analyzed with every batch to ensure the accurate identification and quantitation of validation specimens. A low QC (LQC) was 
prepared at a concentration of $10 \mathrm{ng} / \mathrm{mL}$ and a high QC (HQC) at a concentration of $250 \mathrm{ng} / \mathrm{mL}$. Quality control standards were injected immediately following the final point of the calibration curve and quantitation of each QC was required to be accurate within $20 \%$ of the expected nominal value to proceed with the analysis of validation specimens.

\subsubsection{Sample Preparation}

Meconium samples were accurately weighed and then diluted by a factor of $3(\mathrm{w} / \mathrm{v})$ with 50:50 methanol/water to assist with the sonication procedure. Samples were shaken and sonicated for 10-15 minutes. Following sonication, $1 \mathrm{~mL}$ of the meconium sample was added to appropriately labeled culture tubes. Cocaine, benzoylecgonine and deuterated internal standards were added to samples which were then block vortexed for 5 minutes. Analytes were extracted by first adding $2 \mathrm{~mL}$ of cold acetonitrile while simultaneously vortexing each sample. Samples were then centrifuged for 10 minutes at $3000 \mathrm{rpm}$. Following centrifugation, the organic layer

was transferred to a clean labeled large screw top test tube. $3 \mathrm{~mL}$ of $0.1 \mathrm{M}$ phosphate buffer $(\mathrm{pH}$ 6.0) was added to each sample followed by the addition of $1 \mathrm{~mL}$ of concentrated ammonium hydroxide. Samples were then vortexed by hand for 10-15 seconds followed by a 5 minute centrifugation at $3000 \mathrm{rpm}$.

\subsubsection{Solid Phase Extraction (SPE)}

Analytes were selectively extracted using a solid phase extraction employing UCT cleanscreen ZSDAU020 columns with reversed phase and ion-exchange retention mechanisms. Columns were first conditioned with sequential washes of methanol $(3 \mathrm{~mL})$, deionized water (3 $\mathrm{mL})$ and $0.1 \mathrm{M}$ phosphate buffer, $\mathrm{pH} 6.0(1 \mathrm{~mL})$. All solvents were allowed to drip through the columns slowly under gravity and waste containers were interchanged accordingly. Following column conditioning, samples were poured onto the columns and allowed to drip through 
unassisted for 15 minutes before any pressure was applied. Following sample loading, sequential wash steps were performed using deionized water $(1 \mathrm{~mL}), 1.0 \mathrm{M} \mathrm{HCl}(1 \mathrm{~mL})$, and methanol (3 $\mathrm{mL}$ ). All wash solvents were allowed to drip through the columns unassisted for 5-10 minutes after which time any remaining solvent was assisted through the columns using a positive pressure manifold. Following column wash steps, positive pressure was applied for 5-10 minutes to ensure complete elution of wash solvents. Waste containers were then exchanged for small, labeled elution test tubes and analytes were eluted with $3 \mathrm{~mL}$ of 78:20:2 (dichloromethane: 2propanol: ammonium hydroxide) elution solvent which was made fresh daily. Samples were dried down under a gentle steam of nitrogen and reconstituted in $200 \mu \mathrm{L}$ of DI water:ACN (75:25). Samples were transferred to appropriately labeled plastic vials and injected for analysis.

\subsubsection{Liquid Chromatography}

Liquid chromatographic separations were performed on a Waters ACQUITY ${ }^{\mathrm{TM}}$ ultra performance liquid chromatograph (UPLC ${ }^{\mathrm{TM}}$ ) (Waters Corp., Milford, MA, USA). Separations were achieved on an ACQUITY UPLC ${ }^{\text {TM }}$ HSS T3 column (2.1x 50mm) packed with $1.8 \mu \mathrm{m}$ bridged ethyl hybrid (BEH) particles and maintained at $35^{\circ} \mathrm{C}$. The mobile phase consisted of deionized water containing $0.1 \%$ formic acid (solvent $\mathrm{A}$ ), and acetonitrile containing $0.1 \%$ formic acid (solvent B). Analytes elution was achieved using the following step-wise binary elution gradient: Initial mobile phase composition was 75:25 $\left(\mathrm{H}_{2} \mathrm{O}: \mathrm{ACN}\right)$. Initial conditions were held constant for 0.5 mins after which the composition of solvent B was linearly increased to $50 \%$ over 1.5 mins, finally conditions were returned to their initial composition of 75:25 (H2O:ACN) over the next $0.01 \mathrm{mins}$ and held for $0.49 \mathrm{~min}$ to equilibrate the column before the next injection in the sequence. The total run time was 2.50 mins (Figure 17). Samples were maintained at $7.5^{\circ} \mathrm{C}$ in the sample organizer and sample injection volumes were $5 \mu \mathrm{L}$ for all 
analyses. Flow rates were maintained at $0.5 \mathrm{~mL} / \mathrm{min}$ for the first 0.50 mins after which they were increased to $0.6 \mathrm{~mL} / \mathrm{min}$ for the remainder of the chromatographic separation. All flow was directed into the ESI source of the mass spectrometer.

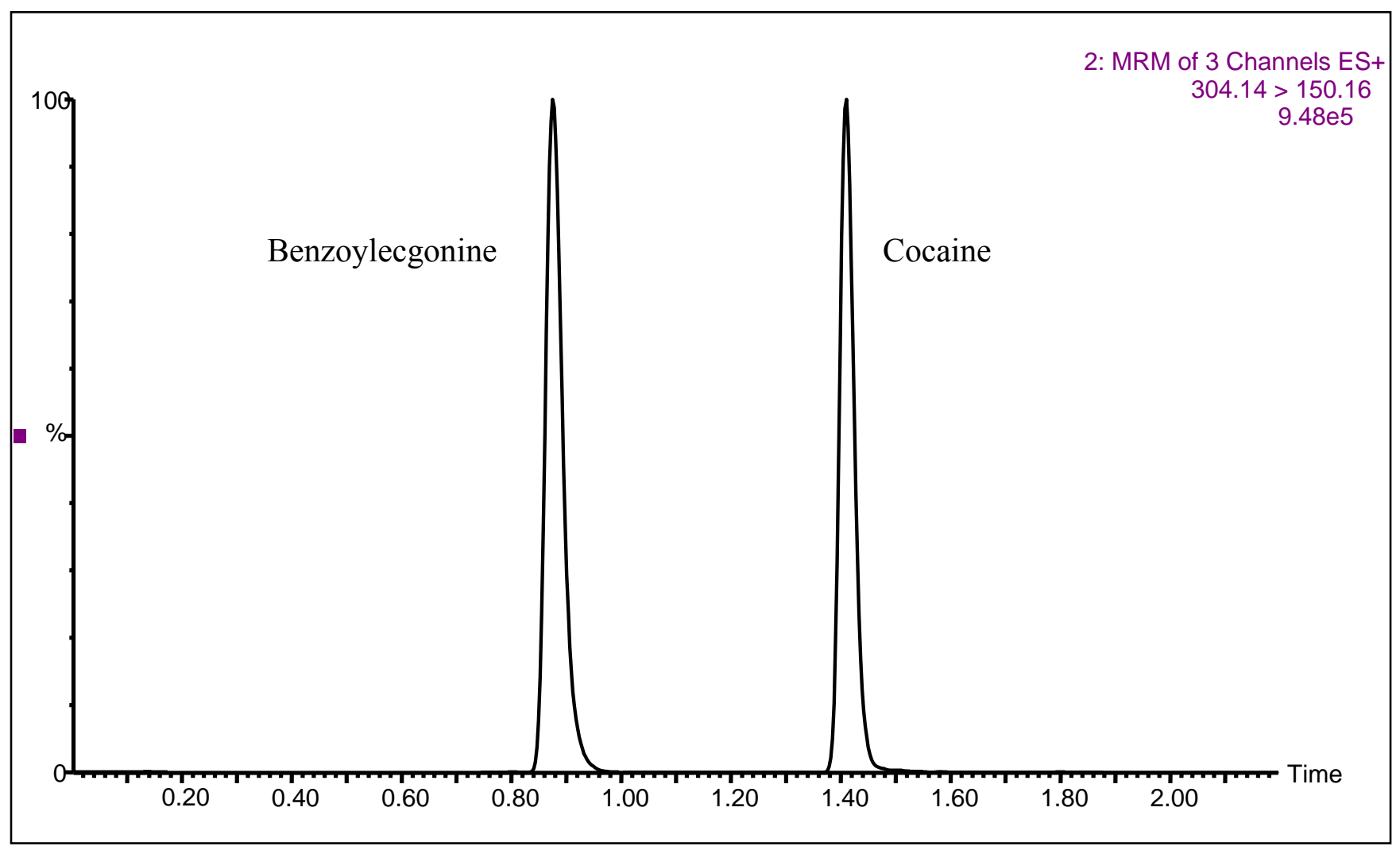

Figure 17: TIC from the UPLC separation of cocaine and benzoylecgonine.

\subsubsection{Mass Spectrometry}

Mass spectrometry conditions were as follows: capillary voltage $0.80 \mathrm{kV}$, cone voltage $20 \mathrm{~V}$, extractor voltage $3.0 \mathrm{~V}, \mathrm{RF}$ lens voltage $0 \mathrm{~V}$. The source temperature was $120^{\circ} \mathrm{C}$ while the desolvation temperature was set at $350^{\circ} \mathrm{C}$. Cone gas was set at a flow of $100 \mathrm{~L} / \mathrm{Hr}$ while the desolvation gas flow was $900 \mathrm{~L} / \mathrm{Hr}$. The collision gas flow was set to $0.10 \mathrm{~mL} / \mathrm{min}$. Nitrogen (99.995\% purity) was used as the desolvation gas, and ultra-pure argon (99.999\% purity) was 
used as the collision gas. Table 12 reports the mass transitions, dwell times, cone voltages, and collision energies for each of the analytes and their deuterated internal standards.

Table 12: MS/MS parameters used for each analyte and deuterated internal standard.

\begin{tabular}{|l|l|l|c|c|c|}
\hline Compound & Mass transition & Purpose & Cone (V) & Collision (V) & Dwell (secs) \\
\hline Cocaine & $304.14>182.10$ & Quantifying ion & 40 & 20 & 0.01 \\
\hline Cocaine & $304.14>150.16$ & Qualifying ion & 40 & 20 & 0.01 \\
\hline Cocaine-d3 & $307.15>184.96$ & Quantifying ion & 30 & 20 & 0.01 \\
\hline Benzoylecgonine & $290.08>168.24$ & Quantifying ion & 40 & 20 & 0.01 \\
\hline Benzoylecgonine & $290.08>104.78$ & Qualifying ion & 40 & 40 & 0.01 \\
\hline Benzoylecgonine-d3 & $293.11>170.98$ & Quantifying ion & 40 & 20 & 0.01 \\
\hline
\end{tabular}

Figure 18 illustrates the fragmentation pathways for cocaine and benzoylecgonine under ESI conditions and the resulting product ions used for analyte quantitation. 


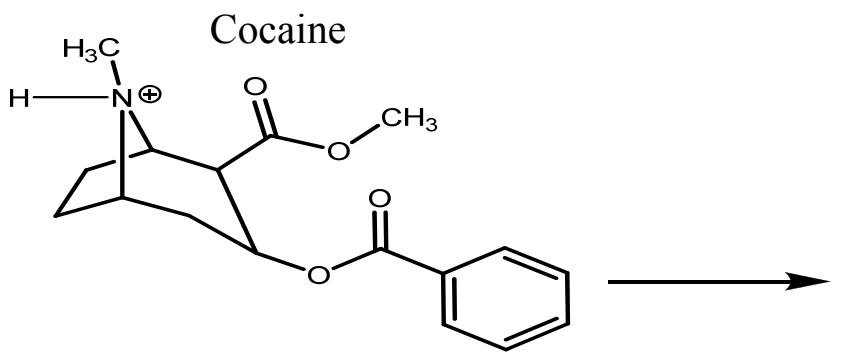

Chemical Formula: $\mathrm{C}_{17} \mathrm{H}_{22} \mathrm{NO}_{4}{ }^{+}$

Molecular Weight: 304.36

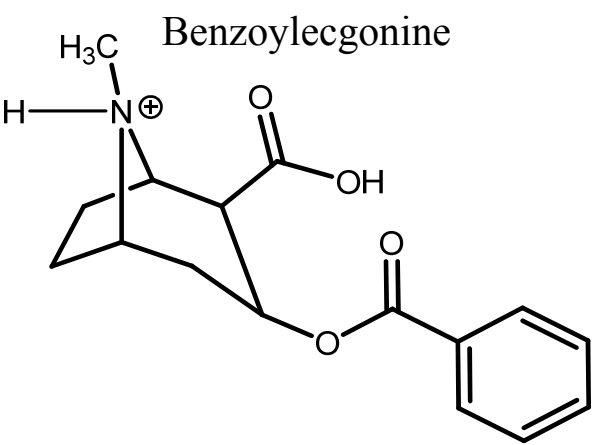

Chemical Formula: $\mathrm{C}_{16} \mathrm{H}_{20} \mathrm{NO}_{4}^{+}$

Molecular Weight: 290.33

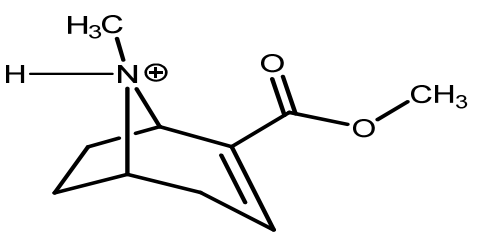

Chemical Formula: $\mathrm{C}_{10} \mathrm{H}_{16} \mathrm{NO}_{2}{ }^{+}$ Molecular Weight: 182.24

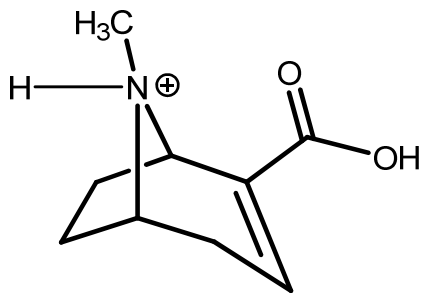

Chemical Formula: $\mathrm{C}_{9} \mathrm{H}_{14} \mathrm{NO}_{2}{ }^{+}$

Molecular Weight: 168.21

Figure 18: Fragmentation pathways for Cocaine and Benzoylecgonine during tandem mass spectrometry experiments[219].

\subsection{Results and Discussion}

\subsubsection{Method Validation}

Preliminary equivalence studies indicated that calibration curves prepared in certified drug-free blood were suitable for the accurate quantification of cocaine and benzoylecgonine in the meconium matrix (Table13 \& 14). The percent relative error (\%RE) calculated for the 5 HQCs and 5 LQCs was less than 1.6\% for cocaine and 7.2\% for benzoylecgonine, indicating a high degree of correlation between the two curves at both the high and low end of the calibration range. Mean relative error values for low quality control specimens were $1.4 \%$, and $3.0 \%$ for 
cocaine and benzoylecgonine, respectively. Mean relative error values for high quality control specimens were $1.3 \%$, and $7.0 \%$ for cocaine and benzoylecgonine, respectively.

Table 13: Cocaine equivalence studies using quantitative values obtained from calibration curves constructed in meconium (MC) and blood (BC).

\begin{tabular}{|c|c|c|c|}
\hline Cocaine Std & *BC (ng/mL) & ${ }^{*} \mathrm{MC}(\mathrm{ng} / \mathrm{mL})$ & $\%$ RE \\
\hline LQC & 14.1 & 14.3 & $1.40 \%$ \\
\hline $\mathrm{HQC}$ & 120.1 & 118.6 & $1.30 \%$ \\
\hline NEG(B) & 0 & 0.2 & N/A \\
\hline NEG(M) & 0.1 & 0.2 & N/A \\
\hline
\end{tabular}

*LQC and HQC concentrations represent mean values of 5 replicate analyses.

Table 14: Benzoylecgonine equivalence studies using quantitative values obtained from calibration curves constructed in meconium (MC) and blood (BC).

\begin{tabular}{|c|c|c|c|}
\hline Benzoylecgonine Std & $*$ BC (ng/mL) & $*$ MC (ng/mL) & \%RE \\
\hline LQC & 15.9 & 16.4 & $3.0 \%$ \\
\hline HQC & 134.7 & 125.9 & $7.0 \%$ \\
\hline NEG(B) & 0 & 0.4 & N/A \\
\hline NEG(M) & 0.1 & 0 & N/A \\
\hline
\end{tabular}

*LQC and HQC concentrations represent mean values of 5 replicate analyses.

The analytical methodology exhibited sufficient selectivity throughout studies using blank meconium specimens and QC standards which had been spiked with various exogenous interferences. Blank meconium specimens were analyzed to ensure that any response generated by the matrix alone corresponded to a concentration less than the LLOQ, while spiked samples were analyzed to assess the ability of the methodology to accurately and precisely quantitate the analyte in the presence of possible exogenous interferences (Table 15). Three blank meconium specimens were spiked with selectivity quality control standards (QCs) which had been previously prepared using various analytes commonly encountered in forensic specimens. The two remaining blank meconium specimens were spiked with opiates (codeine, morphine, hydrocodone, hydromorphone, oxycodone, oxymorphone, and 6-monoacetylmorphine) and THC respectively, at concentrations corresponding to HQCs. The selectivity of the analytical 
methodology in the presence of opiates and THC was investigated as these drugs are commonly encountered in forensic specimens at appreciable concentrations.

Table 15: Exogenous compounds included in selectivity quality control standards.

\begin{tabular}{|c|l|}
\hline Standard & \multicolumn{1}{|c|}{ Contents } \\
\hline Selectivity QC 1 & $\begin{array}{l}\text { Carbamazepine, carbamazepine epoxide, felbamate, gabapentin, lamotrigine, } \\
\text { levetiracetam, mephobarbital, naproxen, oxcarbazepine, phenytoin, primidone, } \\
\text { topiramate, valproic acid, zonisamide }\end{array}$ \\
\hline Selectivity QC 2 & $\begin{array}{l}\text { Amitriptyline, bupropion, clomipramine, desipramine, doxepin, fluoxetine, imipramine, } \\
\text { norclomipramine, nordoxepin, norfluoxetine, norsertraline, nortriptyline, norvenlafaxine, } \\
\text { paroxetine, sertraline, venlafaxine }\end{array}$ \\
\hline Selectivity QC 3 & $\begin{array}{l}\text { Alprazolam, chlorpheniramine, citalopram, clonazepam, cyclobenzaprine, } \\
\text { dextromethorphan, duloxetine, fentanyl, flunitrazepam, haloperidol, mirtazapine, } \\
\text { olanzapine, strychnine, zolpidem }\end{array}$ \\
\hline
\end{tabular}

Blank meconium specimens generated minimal detector responses corresponding to mean analyte concentrations $0.4 \mathrm{ng} / \mathrm{mL}$ for cocaine (Table 16), and $1.5 \mathrm{ng} / \mathrm{mL}$ for benzoylecgonine (Table 17) which were below the LLOQ of $10 \mathrm{ng} / \mathrm{mL}$. Analysis of spiked standards prepared at the LLOQ, indicate that the methodology is selective for the analytes of interest, even in the presence of various exogenous interferences (Table $16 \& 17$ ).

Table 16: Cocaine selectivity studies using blank meconium from five different sources and blank meconium specimens spiked with various exogenous interferences.

\begin{tabular}{|c|c|c|c|c|}
\hline Cocaine Std & Std Conc $(\mathbf{n g} / \mathbf{m L})$ & $\begin{array}{c}* \text { Measured conc } \\
(\mathbf{n g} / \mathbf{m L})\end{array}$ & Acceptable range & Pass/Fail \\
\hline Blank samples & 0 & 0.4 & $<10 \mathrm{ng} / \mathrm{mL}$ & Pass \\
\hline Spiked samples & 10 & 10.3 & $10+/-20 \%$ & Pass \\
\hline
\end{tabular}

*Reported concentrations are mean values of 5 replicate analyses.

Table 17: Benzoylecgonine selectivity studies using blank meconium from five different sources and blank meconium specimens spiked with various exogenous interferences.

\begin{tabular}{|c|c|c|c|c|}
\hline Benzoylecgonine Std & Std Conc (ng/mL) & $\begin{array}{c}{ }^{*} \text { Measured conc } \\
(\mathbf{n g} / \mathbf{m L})\end{array}$ & Acceptable range & Pass/Fail \\
\hline Blank samples & 0 & 1.5 & $<10 \mathrm{ng} / \mathrm{mL}$ & Pass \\
\hline Spiked samples & 10 & 11.6 & $10+/-20 \%$ & Pass \\
\hline
\end{tabular}

*Reported concentrations are mean values of 5 replicate analyses. 
Accuracy studies performed by analyzing five replicates at three different concentrations spanning the calibration range yielded mean values of $254.9 \mathrm{ng} / \mathrm{mL}, 52.7 \mathrm{ng} / \mathrm{mL}$, and 10.5 $\mathrm{ng} / \mathrm{mL}$ reflecting accuracies of $98 \%, 94.6 \%$, and $95 \%$, respectively for cocaine and $245.7 \mathrm{ng} / \mathrm{mL}$, $50.2 \mathrm{ng} / \mathrm{mL}$, and $10.5 \mathrm{ng} / \mathrm{mL}$ reflecting accuracies of $98.3 \%, 99.6 \%$, and $95 \%$, respectively for benzoylecgonine (Table 18 \& 19). Quantitation accuracies were greater than $94 \%$ for both analytes over the entire calibration range.

Table 18: Cocaine accuracy studies.

\begin{tabular}{|c|c|c|c|}
\hline Cocaine Std & Std Conc & "Measured value & \%RE \\
\hline High point & 250 & 254.9 & $2.0 \%$ \\
\hline Midpoint & 50 & 52.7 & $5.4 \%$ \\
\hline LLOQ & 10 & 10.5 & $5.0 \%$ \\
\hline
\end{tabular}

*Reported concentrations are mean values of 5 replicate analyses.

Table 19: Benzoylecgonine accuracy studies.

\begin{tabular}{|c|c|c|c|}
\hline Benzoylecgonine Std & Std Conc & $*$ Measured value & \%RE \\
\hline High point & 250 & 245.7 & $1.7 \%$ \\
\hline Midpoint & 50 & 50.2 & $0.4 \%$ \\
\hline LLOQ & 10 & 10.5 & $5.0 \%$ \\
\hline
\end{tabular}

*Reported concentrations are mean values of 5 replicate analyses.

Both intra-batch and inter-batch precision studies indicated a high degree of precision over the three concentrations investigated with intra-batch CVs ranging from $1.6-9.2 \%$ with the mean being $4.5 \%$ for cocaine (Table 20), while CVs for benzoylecgonine ranged from 5.3-11.8\% with a mean value of 7.4\% (Table 21). Inter-batch precision produced $\mathrm{CV}$ values ranging from $3.9-5.0 \%$ over the three concentration ranges for cocaine and from 4.4-6.2\% over the three concentrations for benzoylecgonine. 
Table 20: Intra- and inter-batch precision studies for cocaine.

\begin{tabular}{|c|c|c|c|c|c|c|}
\hline Cocaine Std & *Day 1 value & *Day 2 value & *Day 3 value & *Day 4 value & 4 day mean & 4 day CV \\
\hline Highpoint & 254.9 & 258.1 & 275.9 & 255.0 & 261.0 & $3.9 \%$ \\
\hline CV & $2.0 \%$ & $1.6 \%$ & $3.5 \%$ & $2.9 \%$ & & \\
\hline Midpoint & 52.7 & 48.3 & 48.8 & 49.6 & 49.9 & $4.0 \%$ \\
\hline CV & $5.2 \%$ & $4.6 \%$ & $4.3 \%$ & $2.4 \%$ & & \\
\hline LLOQ & 10.5 & 10.6 & 10.0 & 9.5 & 10.2 & $4.9 \%$ \\
\hline CV & $9.2 \%$ & $6.8 \%$ & $6.6 \%$ & $4.4 \%$ & & \\
\hline
\end{tabular}

*Reported concentrations are mean values of 5 replicate analyses.

Table 21: Intra- and inter-batch precision studies for benzoylecgonine (BE).

\begin{tabular}{|c|c|c|c|c|c|c|}
\hline BE Std & "Day 1 value & "Day 2 value & *Day 3 value & *Day 4 value & 4 day mean & 4 day CV \\
\hline Highpoint & 245.7 & 264.4 & 245.5 & 230.8 & 246.6 & $5.6 \%$ \\
\hline CV & $6.3 \%$ & $7.9 \%$ & $7.1 \%$ & $5.9 \%$ & & \\
\hline Midpoint & 50.2 & 49.4 & 52.4 & 47.0 & 49.8 & $4.4 \%$ \\
\hline CV & $11.8 \%$ & $5.5 \%$ & $5.9 \%$ & $7.2 \%$ & & \\
\hline LLOQ & 10.5 & 10.5 & 10.9 & 9.4 & 10.3 & $6.2 \%$ \\
\hline CV & $9.5 \%$ & $8.6 \%$ & $7.3 \%$ & $5.3 \%$ & & \\
\hline
\end{tabular}

*Reported concentrations are mean values of 5 replicate analyses.

Analyte recovery was investigated over three concentrations which spanned the calibration range for each analyte and was found to have a mean value of $71.7 \%$ for cocaine (Figure 19) and $10.2 \%$ for benzoylecgonine (Figure 20). Mean recoveries from triplicate analysis at the three concentration ranges investigated ranged from $68.3-77 \%$ for cocaine and $9.3-10.7 \%$ for benzoylecgonine representing good consistency and reproducibility even though the overall recovery of benzoylecgonine was poor. While poor recoveries for benzoylecgonine may be attributable to analyte loss during the solid phase extraction, further studies aimed at determining the degree of recovery during the initial protein precipitation would prove beneficial. 


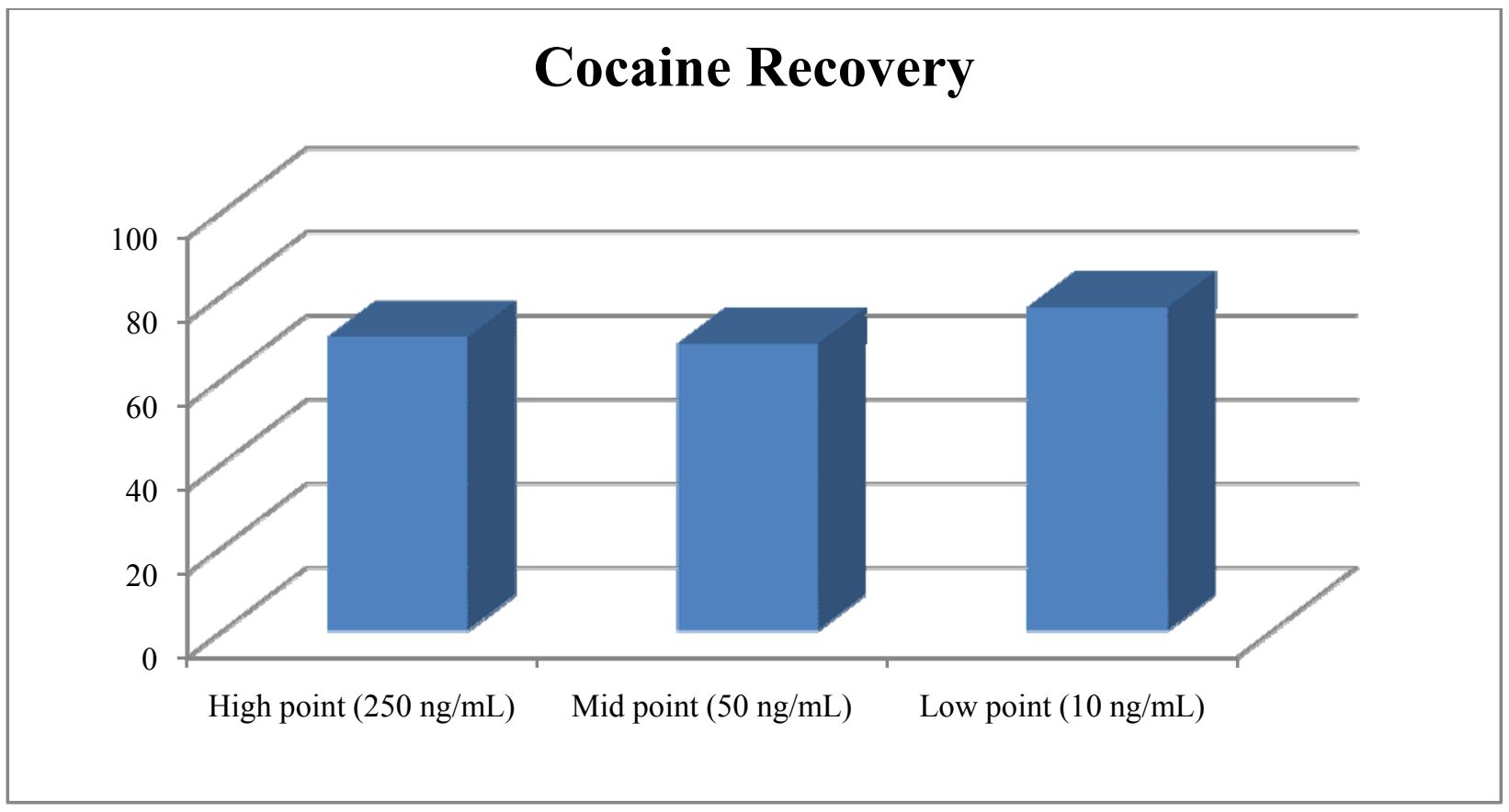

Figure 19: Cocaine recovery at three concentrations over the linear range. 


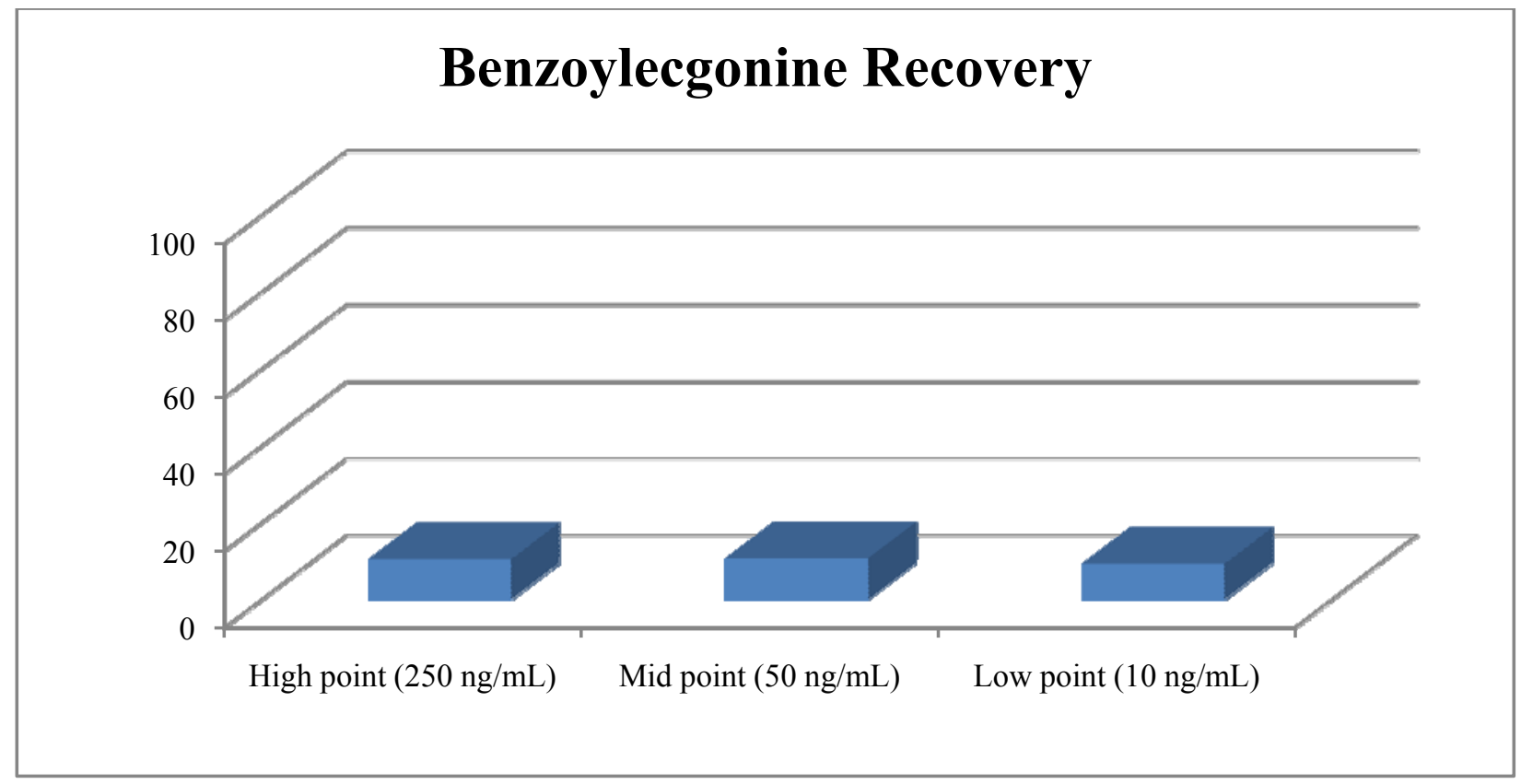

Figure 20: Benzoylecgonine recovery at three concentrations over the linear range.

The limit of detection was determined to be $1 \mathrm{ng} / \mathrm{mL}$ for both cocaine and benzoylecgonine corresponding to a concentration of $4 \mathrm{ng} / \mathrm{g}$ in the meconium specimen prior to sonication. Unequivocal identification of analytes was not feasible at concentrations below $1 \mathrm{ng} / \mathrm{mL}$ due to significant fluctuation and inaccuracies in calculated ion ratios. Both cocaine and benzoylecgonine appear to be quite stable in the meconium matrix over the investigated calibration range when stored at $4^{\circ} \mathrm{C}$. Quantitation accuracies during stability studies were greater than $84 \%$ and $93 \%$ for cocaine HQC and LQCs respectively, and greater than $95 \%$ and 99\% for benzoylecgonine HQC and LQCs respectively. 


\subsection{Conclusions}

Development and validation of an accurate, selective, precise, and sensitive UPLC-ESIMS/MS method for the identification and quantification of cocaine and benzoylecgonine in meconium was achieved. For the purpose of improved selectivity, two MRM transitions were monitored for each analyte and quantifications was achieved using deuterated internal standards and a six point calibration curve. Injection volumes of $5 \mu \mathrm{L}$ allowed for the accurate quantification of both analytes over the range $10-250 \mathrm{ng} / \mathrm{mL}$, corresponding to concentrations of $40-1000 \mathrm{ng} / \mathrm{g}$ in the original meconium specimens. Significant gains in sample throughput are achieved with ultra performance liquid chromatography through rapid chromatographic separations made possible by sub $2 \mu \mathrm{m}$ packing materials. This increased speed of analysis alleviates problems associated with extensive sample preparation and allows working toxicology laboratories to increase sample throughput without sacrificing the quality of the data. To the best of the author's knowledge, this is the first report of a validated UPLC-ESI-MS/MS method for the quantitative analysis of cocaine and benzoylecgonine in meconium specimens. The validated methodology was implemented at AIT Laboratories (Indianapolis, IN) for the purpose of confirming the presence of cocaine and benzoylecgonine in authentic meconium specimens which had previously screened positive. From 07/01/08 - 03/06/2009 a total of 4036 meconium specimens were screened for cocaine/benzoylecgonine. Of those samples, 303 screened positive

representing $7.5 \%$ of the total specimens. 71 of the 303 specimens were confirmed positive using the UPLC-MS/MS method. 


\section{Chapter 6: Detection and Quantification of Codeine, Morphine, Hydromorphone, and 6-Monoacetylmorphine (6-MAM) in Meconium using ISOLUTE HM-N Supported-Liquid Extraction Columns and UPLC-MS/MS}

\subsection{Abstract}

A method for the extraction and quantification of codeine, morphine, hydromorphone, and 6-monoacetylmoprhine (6-MAM) from meconium specimens using supported-liquid extraction (SLE) columns and ultra performance liquid chromatography coupled to tandem mass spectrometry (UPLC-MS/MS) is described.

Deaths resulting from heroin (diacetylmorphine) use have long been considered an epidemic and continue to plague society due to the addictive nature and abuse potential of the drug. While heroin remains a popular drug of abuse in today's society, large increases in the prescriptive medical use of several opiate analgesics over the past ten years have been accompanied by increases in morbidity due to abuse, addiction, and diversion. Popular opiate analgesics commonly prescribed for the treatment of pain include morphine, codeine, hydrocodone, and hydromorphone.

Meconium specimens were sonicated to maximize homogeneity of the sample and analytes were subsequently extracted from the matrix using ISOLUTE HM-N supported-liquid extraction (SLE) columns. Quantitation was performed using a Waters UPLC ${ }^{\mathrm{TM}}$ chromatograph coupled to a triple quadrupole mass spectrometer equipped with an electrospray ionization (ESI) source. Chromatographic separation was achieved in less than 5 minutes, data was acquired in the MRM mode and quantitation was achieved using multi point calibration curves. 
The analytical methodology was deemed selective for the analyte of interest in the presence of exogenous interferences. Quantitation accuracy ranged from $90-100 \%$ for all four analytes over three concentrations spanning the calibration range. Intra-batch precision studies indicated high quantitative precision with $\mathrm{CV}$ values ranging from $1.2-10.7 \%$ for all analytes. Inter-batch precision was high over the entire calibration range with $\mathrm{CV}$ values ranging from 0.5 $-6.1 \%$. Analyte recovery ranged from $52.3-76.3 \%$ for all four analytes representing good extraction efficiencies. The limit of detection for all four analytes was $2.5 \mathrm{ng} / \mathrm{mL}$, corresponding to a concentration of $10 \mathrm{ng} / \mathrm{g}$ prior to homogenization.

By employing ISOLUTE HM-N supported-liquid extractions for the isolation of analytes in meconium, sample preparation time is greatly reduced without sacrificing analyte recovery. Ultra performance liquid chromatography allowed for the rapid chromatographic separation of all four analytes in less than 5 minutes and tandem mass spectrometric detection ensured increased sensitivity and selectivity relative to traditional techniques such as HPLC-UV and GC/MS. Development of analytical techniques requiring minimal sample preparation for the determination of opiates in meconium specimens will aid in the identification of infants prenatally exposed to drugs such as heroin, morphine, codeine, hydrocodone, and hydromorphone.

\subsection{Mechanism of Opiate Action}

Members of the opiate family mimic the effects of endogenous analgesics termed 'endogenous opioid peptides,' which include $\beta$-endorphin, leu-enkephalin, met-enkephalin, dynorhpins, and the neoendorphins[220]. Such peptides are released by the body to regulate pain and it is this action which is mimicked by members of the opiate family which bind to specific receptors in the spinal cord and prevent the release of excitatory neurotransmitters[220]. In 
combination to directly blocking the release of excitatory neurotransmitter in the spinal cord, opiates also act to inhibit the release of neurotransmitters in certain nerve fibers which are responsible for relaying pain signals to the spinal cord. The combination of central and peripheral inhibition results in a powerful analgesic effect common to all members of the drug class[220].

The identification of specific receptors at which opiates bind was not reported until 1976 when Martin and coworkers performed a detailed analysis of the neurophysiological and behavioral properties of several opiate compounds looking specifically for cross tolerance[221]. Studies suggested the presence of three types of opioid receptors which were subsequently named after the drugs used in the studies. The $\mu(\mathrm{mu})$ receptor was named after morphine, while the $\kappa$ (kappa) receptor was named after ketocyclazocine and the $\sigma$ (sigma) receptor after Nallylnormetazocine. The $\sigma$ receptor is no longer considered an opiate receptor. In 1977, Kosterlitz and coworkers identified the $\delta$ (delta) opiate receptor when they noticed that action of enkephalins on the vas deferens was relatively insensitive to naloxone[222].

The existence of such receptors has always been theorized due mainly to the stereoselectivity of the opiates, the existence of antagonists and the differences in potencies throughout the opiate family. As already mentioned, opiates act by binding to one or multiple of the opiate receptors thereby preventing the transmission of second messenger signals associated with pain. Although the actual biochemical mechanism associated with this process is not fully understood, it is thought to involve changes in the coupling of a G-protein to the regulatory enzyme adenylate cyclase which in turn inhibits the actions of adenylate cyclase. Inhibition of the adenylate cyclase enzyme facilitates the opening of potassium $\left(\mathrm{K}^{+}\right)$ion channels and the simultaneous closing of calcium $\left(\mathrm{Ca}^{++}\right)$channels which results in the hyperpolarization of 
neurons and the overall regulation of pain[223]. Most of the commonly prescribed opiates are full agonists at the mu receptor and many members of the opiate family act on all three receptors (Table 22).

Table 22: Receptor activity for selected opiates and synthetic derivatives.

\begin{tabular}{|c|c|c|c|c|}
\hline Opiate & Overall relationship & $\begin{array}{c}\boldsymbol{\mu}(\mathbf{m u}) \text { receptor } \\
\text { action }\end{array}$ & $\begin{array}{c}\boldsymbol{\delta} \text { (delta) receptor } \\
\text { action }\end{array}$ & $\begin{array}{c}\boldsymbol{\kappa} \text { (kappa) receptor } \\
\text { action }\end{array}$ \\
\hline Morphine & full agonist & strong agonist & weak agonist & weak agonist \\
\hline Codeine & full agonist & weak agonist & weak agonist & no activity \\
\hline Methadone & full agonist & strong agonist & weak agonist & weak agonist \\
\hline Fentanyl & full agonist & strong agonist & weak agonist & no activity \\
\hline Buprenorphine & partial agonist & partial agonist & no activity & antagonist \\
\hline Pentazocine & partial agonist & antagonist & weak agonist & moderate agonist \\
\hline Naloxone & antagonist & antagonist & antagonist & antagonist \\
\hline
\end{tabular}

Agonists for any of the three opiate receptors will possess some degree of analgesic inducing properties however additional side effects will vary somewhat depending on the individual receptor. Mu-specific agonists will cause powerful analgesia along with the euphoria responsible for the widespread abuse of opiates. The mu receptor is also responsible for many of the unwanted effects of opiates such as respiratory depression which is considered to be the main side effect of opiates associated with most overdoses. Drug induced respiratory depression occurs when the brain is unaware of accumulating levels of $\mathrm{CO}_{2}$ and subsequently understimulates the lung musculature[224]. The brain is unaware of such accumulation due to desensitization of the medulla which is responsible for determining the need to breathe deeper, faster, and more rhythmically in times of $\mathrm{CO}_{2}$ accumulation[224]. In addition to inducing respiratory depression, agonists at the mu receptor also produces miosis, reduction in motility of the GI system, and physiological dependence[220]. Agonists at the kappa receptor will induce 
spinal analgesia, dysphoria, and sedation while opiate agonists at the delta receptor will mediate analgesia, additional respiratory depression, slight euphoria and dependence[220].

\subsection{Introduction to Commonly Abused Opiates}

The opiates are a large family of drugs which are structurally and pharmacologically related to morphine. Morphine is the central member of the opiate family and is obtained from raw opium, which is the dried sap of the seed capsule in the opium poppy (Papaver somniferum). Raw opium is initially purified to refined opium which is a sticky gum exudate containing many alkaloids including morphine $(\sim 10 \%)$, codeine $(\sim 0.5 \%)$ and thebaine $(\sim 0.4 \%)$ which are subsequently extracted and prepared for medicinal purposes[220, 225]. Members of the opiate family include several semi-synthetic compounds such as codeine, oxycodone, dihydrocodeine, hydrocodone, and hydromorphone, which are derived from the morphine molecule through chemical modification[220].

Several synthetic opiates, known more commonly as opioids, such as fentanyl, methadone, meperidine, propoxyphene and buprenorphine are also commonly prescribed for their analgesic properties. Although these compounds appear structurally unique relative to the morphine molecule, their three dimensional interaction with opioid receptors is very similar. Although the exact mechanism by which opiates act on the central nervous system (CNS) is still largely speculative, their pharmacological effect has been documented and sought after for over two hundred years[226].

The interaction of opiates with specific receptors in the CNS is responsible for their central effects such as analgesia, drowsiness, mental dullness, changes in mood, and euphoria[220, 225, 227]. The pharmacological activity of opiates mimics that of endogenous 
opioid peptides which are produced naturally in the body and interact with receptors throughout the nervous system and GI tract to alter the body's response to painful stimuli[227]. Because of their ability to modulate pain and reduce suffering, opiates are an extremely important and widely prescribed class of drug throughout many fields of medicine. The ability of opiates and certain illicit derivatives (eg. heroin) to produce feelings of euphoria, also means that they are one of the most widely abused drug classes in today's society.

In 1999, the federally sponsored Drug Abuse Warning Network (DAWN) program indicated that heroin toxicity accounted for almost half (42\%) of all narcotic-related deaths and $41.3 \%$ of all reported drug-related deaths[204]. In 2006, a National Vital Statistics Report published by the US Department of Health and Human Services indicated that heroin related deaths were continuing to increase and that a total of 2,101 deaths were attributed to heroin overdose in 2002, representing a $17 \%$ increase from the previous year[228, 229]. As opiate related deaths continue to increase throughout the world, heroin is still considered the most prevalent opiate of interest in post mortem toxicology due to its widespread availability and ability to induce life threatening respiratory depression[230]. Following administration, heroin is rapidly converted via deacetylation to 6-monoacetylmorphine (6-MAM) and then to morphine (Figure 21)[204]. 


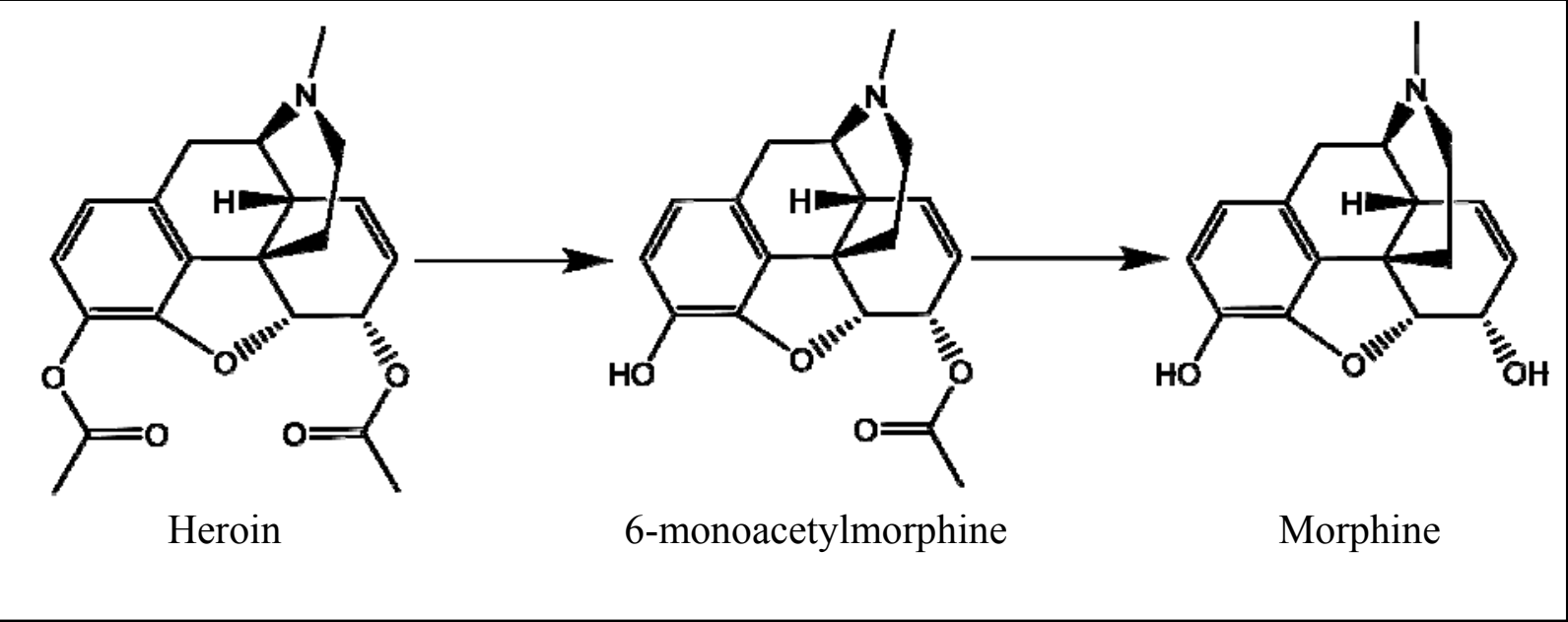

Figure 21: Metabolism of heroin.

The conversion of heroin to 6-monoacetlymorphine is complete within 10-15 minutes while the total conversion of heroin to morphine is completed within a few hours [204]. Due to its rapid biochemical transformation following administration, heroin has a similar excretion profile to morphine, although small amounts of 6-MAM are found in the urine of most heroin users[220]. The presence of 6-MAM in the urine distinguishes heroin use from morphine use and although the presence of trace levels of codeine are also consistent with heroin use (due to the presence of codeine in the heroin as an impurity), only the presence of 6-MAM can unequivocally identify the source of morphine as heroin. 6-MAM has also been shown to cross the placental membrane and accumulate in the meconium of infants exposed to heroin prenatally, and similar to urine, its identification in meconium provides definitive proof of maternal heroin use[92]. The detection of 6-MAM in the meconium of newborn infants is vital to distinguish morphine use from heroin use.

Morphine is administered to pregnant women for several reasons such as the induction of spinal anesthesia[231], for the treatment of acute pancreatitis[232], and to provide improved labor and postcesarean analgesia[233] and while most sources of morphine will be administered 
only hours prior to birth and will likely not present in the meconium of the newborn, the presence of 6-MAM will distinguish heroin use from any morphine use, licit or otherwise.

Morphine (MS Contin, Kadian, Avinza) is one of the most widely prescribed opiates for the treatment of chronic pain due to its highly potent analgesic effects. Morphine is likely the most effective opiate for the treatment of severe pain and while its analgesic effects are well utilized in the field of pain management, its potent agonist activity at the mu receptor also induces euphoric effects which are sought after by addicts worldwide.

Joranson and coworkers investigated trends in the medical use of morphine for the treatment of severe pain over a six year period. In 1990, approximately 2.2 million grams of morphine were prescribed to patients suffering from severe pain. Over the next six years, this number increased by 59\% and by 1996, 3.5 million grams of morphine were being actively prescribed to patients across north America[234]. Although the authors conclude that the increasing use of medical morphine did not appear to contribute to the increasing health consequences of opiate analgesic abuse, a separate study published in the Journal of Addictive Diseases reports that the rate of morphine prescription (number of morphine prescriptions per 10,000 patient encounters) increased 2.64 fold over the time period 1995-2004, while the rate of emergency department (ED) mentions for morphine simultaneously increased 1.16 fold[235].

Since 1995, there has been an overall $86 \%$ increase in morphine use across the United States[235]. Morphine is largely metabolized by glucuronidation at the 3- and 6-positions to form morphine-3-glucuronide and morphine-6-glucuronide respectively, the latter of which has a similar pharmacological activity to morphine (Figure 22). 


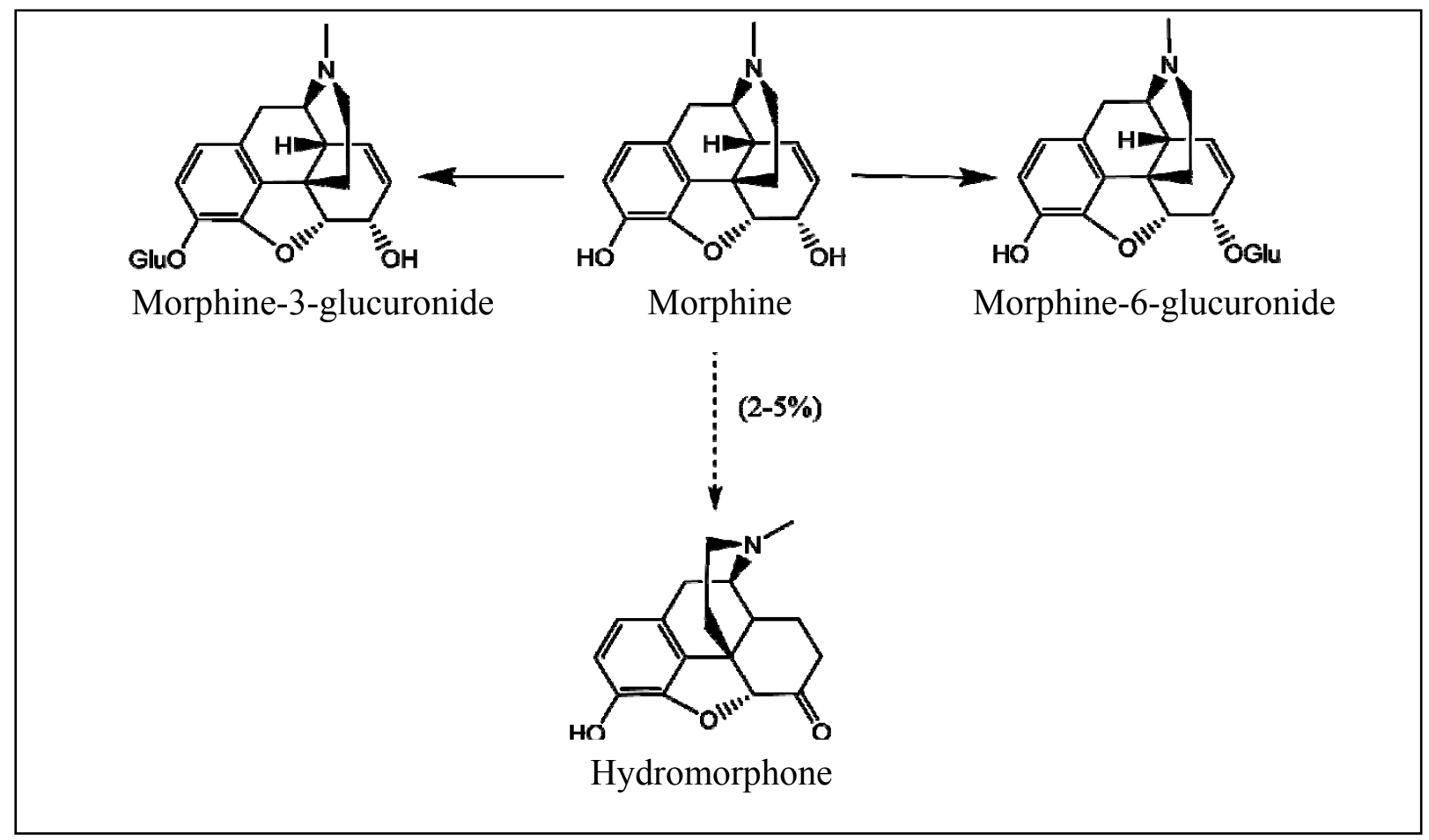

Figure 22: Biotransformation and conjugation of morphine.

Both free and conjugated forms of morphine have been shown to cross the placental barrier and accumulate in the meconium of prenatally exposed newborns[92]. Recent studies also suggest that morphine undergoes a minor metabolic transformation to hydromorphone in the cases of high dose, steady state patients (Figure 22)[236, 237]. Hydromorphone is considered a secondary metabolite of morphine that will not necessarily be detectable in the case of an acute overdose but may be present in the urine of patients taking large doses over significant periods of time at concentrations corresponding to $2-5 \%$ of the morphine. Hydromorphone has also been shown to cross the placental barrier and accumulate in the meconium of infants prenatally exposed to the drug or one of its metabolic precursors such as hydrocodone and morphine[92].

Hydrocodone (Vicodin, Lortab, Lorcet) is a popular, short acting, opiate analgesic prescribed for the treatment of mild pain and often formulated with non-opiate analgesics such as 
acetaminophen or salicylates. Such formulations provide increased analgesia via drug synergy and also to deter patients from overdosing by formulating the opiate with NSAIDS which have unpleasant side effects when taken in high doses.

Wisniewski et al. investigated the relationship between the prescribing trends for hydrocodone products and the aggregate indicators of non-medical abuse such drug induced and drug-related emergency department (ED) visits, over an eight year time period[235]. Studies indicated that the number of prescriptions written per 10,000 patients increased 2.03 fold over the eight year period while the number of hydrocodone-related ED visits increased 1.60 fold[235]. The rate of hydrocodone use has consistently increased since 1999 and in 2005, more prescriptions were written for hydrocodone/acetaminophen combination products than any other medication including drugs such as amoxicillin and atorvastatin[238].

Hydrocodone is metabolized in man by $\mathrm{O}-$ and $\mathrm{N}$-demethylation and reduction of the 6keto group[239]. Hydrocodone, like many drugs is metabolized by a large family of enzymes know as the cytochrome P450 (or CYP450) enzymes. The 2D6 isozyme of the CYP450 family, known as CYP2D6 is responsible for the O-demethylation of hydrocodone to hydromorphone, which is also a potent opiate analgesic prescribed for the treatment of pain (Figure 23). 


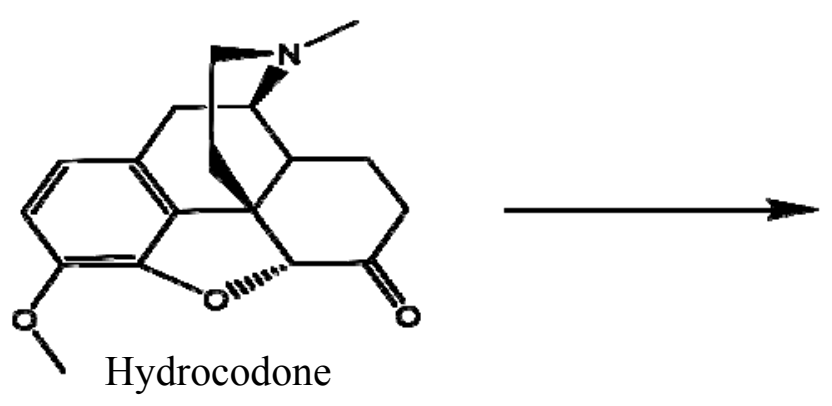

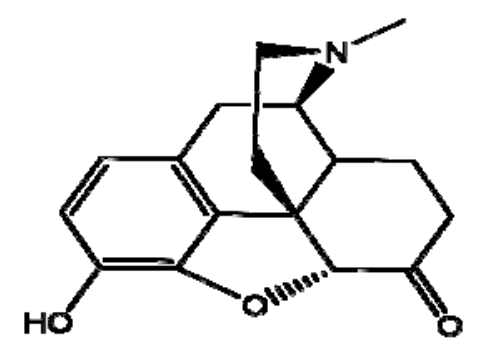

Hydromorphone

Figure 23: Metabolism of hydrocodone to hydromorphone.

Rapid metabolizers have been shown to produce and excrete significantly more of a dose as hydromorphone than poor metabolizers[239].

Hydromorphone (Dilaudid) is a strong opiate analgesic prescribed for the treatment of chronic pain. Hydromorphone is also the active metabolite of hydrocodone which undergoes Odemethylation by CYP2D6 to form the active metabolite. Joranson and coworkers reported a $19 \%$ increase in the medical use of hydromorphone from 1900 to 1996 at which time 141,325 grams of the drug were being prescribed across the united states[234]. Hydromorphone is a potent opiate which has been responsible for fatalities at concentrations as low as $51 \mathrm{ng} / \mathrm{mL}$ and a particular review of hydromorphone related deaths in Ontario from 1985 to 2003 revealed that deaths attributed to hydromorphone toxicity involved concentrations ranging from 77 to 2684 $\mathrm{ng} / \mathrm{mL}[240]$. Hydromorphone abuse is increasing in today's society and its detection in meconium is of additional importance as it will also assist in identifying maternal hydrocodone use.

Prenatal exposure to opiates has been associated with the following symptoms in infants: colic pains, agitation, irritability, high pitched cries, and long term withdrawal symptoms known as prenatal opiate withdrawal[241, 242]. 
Table 23 provides the chemical structures of morphine, codeine, hydromorphone, and 6monacetylmorphine along with certain physicochemical properties such as molecular weights, and pKa's.

Table 23: Chemical structures, molecular weights, molecular formulas, and pKa's of morphine, codeine, hydromorphone, and 6-monoacetylmorphine.

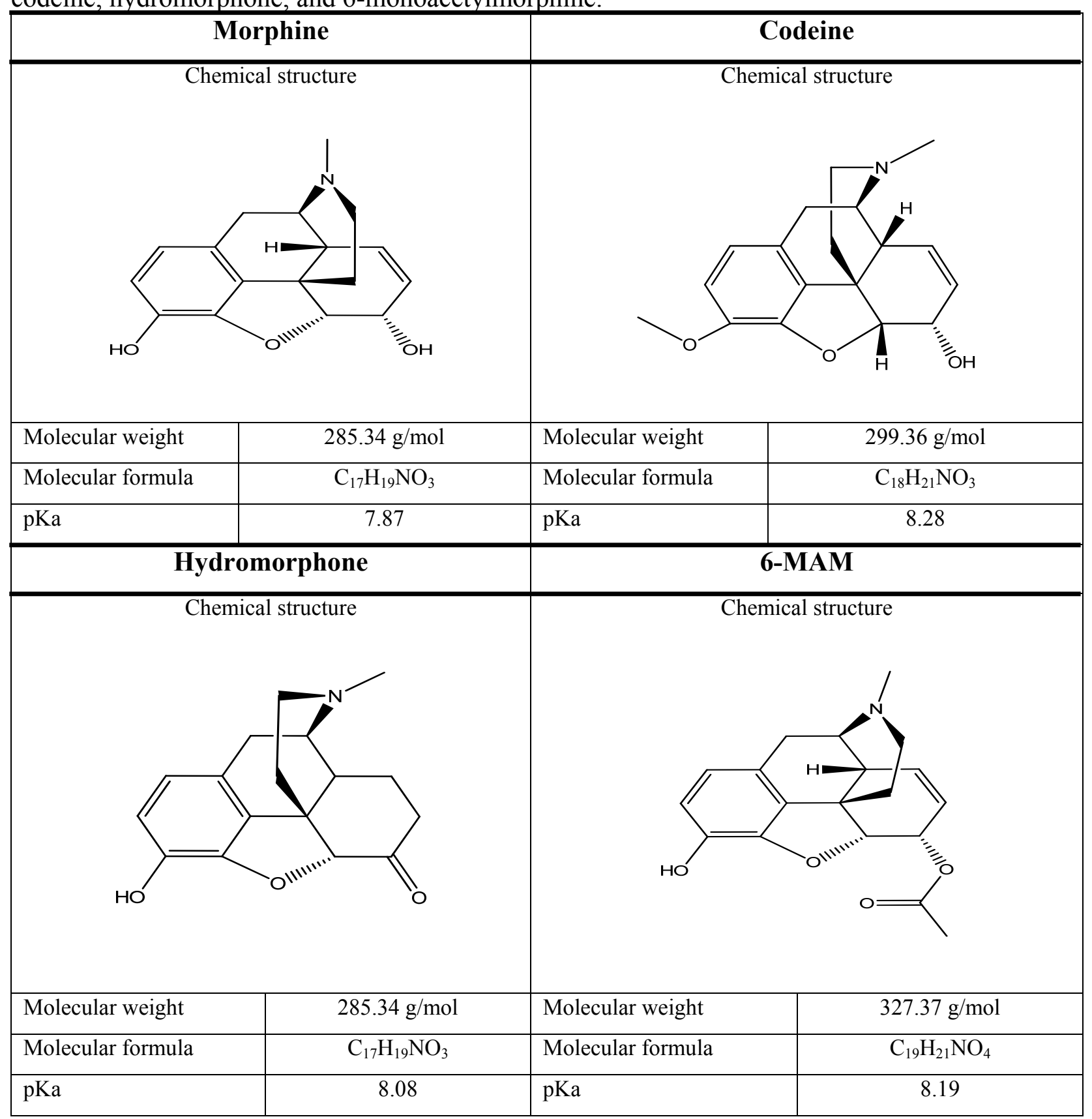




\subsection{History of Opiate Detection in Meconium}

The detection of opiates in meconium has been the focus of far fewer research efforts than that of cocaine. The detection of opiates in meconium is becoming increasingly important as prescription opiates such as morphine, codeine, and hydrocodone are being abused as a result of their widespread availability on the street and cheap cost. Analytical methodologies capable of detecting opiates in meconium have been largely based on GC/MS technologies due to the selectivity afforded by MS.

Moriya and co-workers successfully detected the presence of morphine in the stool of a 41 day old infant at a concentration of $1340 \mathrm{ng} / \mathrm{g}$ following derivatization with trifluoroacetic anhydride and GC/MS analysis[85]. In an effort to increase the selectivity and sensitivity of meconium analysis which had been largely performed using radioimmunoassays, ElSohly et al employed GC/MS for the determination of several drug classes, including opiates, in meconium[243]. Pichini and co-workers employed an ESI-LC/MS assay operating in SIM mode for the simultaneous determination of opiates and cocaine in meconium. Analytes were extracted using an initial liquid extraction into methanol or ammonium hydrogen carbonate buffer followed by a solid phase extraction for subsequent sample clean-up. Method validation included a linear dynamic range of $0.005-1.00 \mu \mathrm{g} / \mathrm{g}[217]$.

Coles and co-workers reported the first tandem mass spectrometry method for the determination of codeine, morphine, hydrocodone, hydromorphone, oxycodone, and 6acetylmorphine in meconium following liquid chromatographic separation. A decreased sample preparation time was achieved by eliminating the glucuronide hydrolysis step and employing a solid phase extraction in which the elution occurred directly into autosampler vials. LC-MS/MS 
provided enhanced selectivity for the detection of the six opiates with a decreased interference rate of $3.9 \%$ compared to $13.6 \%$ for a previously established GC/MS method[92].

To the best of the author's knowledge, this is first report of the simultaneous detection of codeine, morphine, hydromorphone and 6-monoacetylmorphine in meconium using UPLCMS/MS. The implementation of UPLC methodologies into routine meconium analysis will increase the speed and efficiency of chromatographic separations allowing for more rapid separation of various opiate derivatives without the need for complex mobile phases. Combining the chromatographic advantages of UPLC with the increased selectivity of tandem mass spectrometry will result in more rapid, accurate, and sensitive identifications of various opiate derivatives in human meconium.

\subsection{Experimental}

\subsubsection{Chemicals and reagents}

Codeine, morphine, hydromorphone, 6-monoacetylmorhine, codeine- $\mathrm{d}_{3}$, morphine- $\mathrm{d}_{3}$, hydromorphone- $\mathrm{d}_{3}$, and 6-monoacetylmorphine- $\mathrm{d}_{6}$ standards $(1 \mathrm{mg} / \mathrm{mL}$ in methanol) were obtained from Cerilliant (Round Rock, TX). ISOLUTE ${ }^{\circledR}$ HM-N supported liquid-liquid extraction columns were purchased from Biotage (Charlottesville, VA). All solvents were HPLC grade and obtained from Fisher Scientific (Pittsburgh PA).

\subsubsection{Calibration Curve Matrix}

Meconium specimens which had previously screened negative for opiates using a $50 \mathrm{ng} / \mathrm{g}$ cutoff at AIT laboratories (Indianapolis, IN) were collected and spiked with codeine, morphine, hydromorphone, and 6-monoacetylmorphine to give concentrations of $10 \mathrm{ng} / \mathrm{mL}(\mathrm{n}=5)$ and 500 $\mathrm{ng} / \mathrm{mL}(\mathrm{n}=5)$. Spiked meconium was then quantified using a calibration curve constructed in 
negative meconium and a calibration curve constructed in certified drug free whole blood.

Quantitative results obtained using the meconium calibration curve showed excellent correlation $(<15 \% \mathrm{CV})$ with those obtained using the calibration curve made up in negative blood and as a result, all subsequent method validation experiments were performed using calibration curves prepared in certified negative blood.

\subsubsection{Working Standards}

Calibration curves were constructed using eight calibration standards prepared in certified drug free whole blood and all sample volumes were $1 \mathrm{~mL}$. Two working standards were used to construct the calibration standards, working standard $1(500 \mathrm{ng} / \mathrm{mL})$ was prepared by combining $150 \mu \mathrm{L}$ of an amphetamine and methamphetamine stock solution $(10 \mu \mathrm{g} / \mathrm{mL})$ with $2850 \mu \mathrm{L}$ of negative blood. Working standard $2(50 \mathrm{ng} / \mathrm{mL})$ was prepared by combining $300 \mu \mathrm{L}$ of working standard 1 with $2700 \mu \mathrm{L}$ of negative blood. An internal standard solution $(500 \mathrm{ng} / \mathrm{mL})$ was prepared using $1 \mathrm{mg} / \mathrm{mL}$ standards of codeine- $\mathrm{d}_{3}$, morphine- $\mathrm{d}_{3}$, hydromorphone- $\mathrm{d}_{3}$, and 6monoacetylmorphine- $\mathrm{d}_{6}$.

\subsubsection{Calibration Curves}

Calibration curves for all experiments were prepared according to Table 24 .

Table 24: Preparation of codeine, morphine, hydromorphone, and 6-monacetylmorphine calibration curves.

\begin{tabular}{|c|c|c|}
\hline Standard Concentration $(\mathbf{n g} / \mathbf{m L})$ & Volume of Working Standard $(\boldsymbol{\mu} \mathbf{L})$ & Volume of Negative Blood $(\boldsymbol{\mu} \mathbf{L})$ \\
\hline 500 & $1000(\mathrm{Std})$ & 0 \\
\hline 250 & $500(\mathrm{Std})$ & 500 \\
\hline 100 & $200(\mathrm{Std})$ & 800 \\
\hline 50 & $1000(\mathrm{Std} 2)$ & 0 \\
\hline 25 & $500(\mathrm{Std})$ & 500 \\
\hline 10 & $200(\mathrm{Std} 2)$ & 800 \\
\hline 5 & $100(\mathrm{Std} 2)$ & 900 \\
\hline Negative & 0 & 1000 \\
\hline
\end{tabular}


$100 \mu \mathrm{L}$ of internal standard solution $(500 \mathrm{ng} / \mathrm{mL})$ was added to each sample including the blank and all samples were then vortexed for 30 seconds.

\subsubsection{Quality Control (QC) Standards}

Quality control standards (QC's) were prepared and analyzed with every batch to ensure the accurate identification and quantitation of validation specimens. A low QC (LQC) was prepared at a concentration of $10 \mathrm{ng} / \mathrm{mL}$ and a high QC (HQC) at a concentration of $500 \mathrm{ng} / \mathrm{mL}$. Quality control standards were injected immediately following the final point of the calibration curve and quantitation of each QC was required to be accurate within $20 \%$ of the expected nominal value to proceed with the analysis of validation specimens.

\subsubsection{Sample Preparation}

Meconium samples were accurately weighed and then diluted by a factor of $3(\mathrm{w} / \mathrm{v})$ with 50:50 methanol/water to assist with the sonication procedure. Samples were shaken and sonicated for 10-15 minutes. Following sonication, $1 \mathrm{~mL}$ of the meconium samples was added to appropriately labeled culture tubes. Codeine, morphine, hydromorphone, and 6monoacetylmorphine standards and deuterated internal standards were added to samples which were then block vortexed for 5 minutes. Samples were centrifuged for 5 minutes at $3500 \mathrm{rpm}$ after which the supernatants were transferred to appropriately labeled culture tubes (12x75). Samples were then diluted with $2 \mathrm{~mL}$ of deionized water and vortexed for $10-15$ seconds. Samples were loaded into 5mL ISOLUTE HM-N supported liquid extraction columns and left to sit for 10 minutes. Analytes were initially eluted with $5 \mathrm{~mL}$ of ethyl acetate and after a 3 minute waiting period a second elution step was performed with $3 \mathrm{~mL}$ of ethyl acetate. Samples were dried down under a gentle stream of nitrogen and reconstituted in $200 \mu \mathrm{L}$ of DI water. Samples were transferred to UPLC vials and injected. 


\subsubsection{Liquid Chromatography}

Liquid chromatographic separations were performed on a Waters ACQUITY ${ }^{\mathrm{TM}}$ ultra performance liquid chromatograph (UPLC ${ }^{\mathrm{TM}}$ ) (Waters Corp., Milford, MA, USA). Separations were achieved on an ACQUITY UPLC ${ }^{\text {TM }}$ HSS T3 column $(2.1 \times 50 \mathrm{~mm})$ packed with $1.8 \mu \mathrm{m}$ bridged ethyl hybrid $(\mathrm{BEH})$ particles and maintained at $35^{\circ} \mathrm{C}$. The mobile phase consisted of deionized water containing $0.1 \%$ formic acid (solvent A), and acetonitrile containing $0.1 \%$ formic acid (solvent B). Analyte elution was achieved using the following step-wise binary elution gradient: Initial mobile phase composition was 97:3 ( $\left.\mathrm{H}_{2} \mathrm{O}: \mathrm{ACN}\right)$. The composition of solvent B was increased to $20 \%$ over the first 2.50 mins and then rapidly increased to $99 \%$ over the next 0.05 mins where it was held constant for 0.45 mins, finally conditions were returned to their initial composition of 97:3 (H2O:ACN) over the final 0.01 mins of the chromatographic run. The total run time was 3.01 minutes (Figure 24). Samples were maintained at $7.5^{\circ} \mathrm{C}$ in the sample organizer and sample injection volumes were $5 \mu \mathrm{L}$ for all analyses. Flow rates remained constant at $0.6 \mathrm{~mL} / \mathrm{min}$ and all flow was directed into the ESI source of the mass spectrometer. 


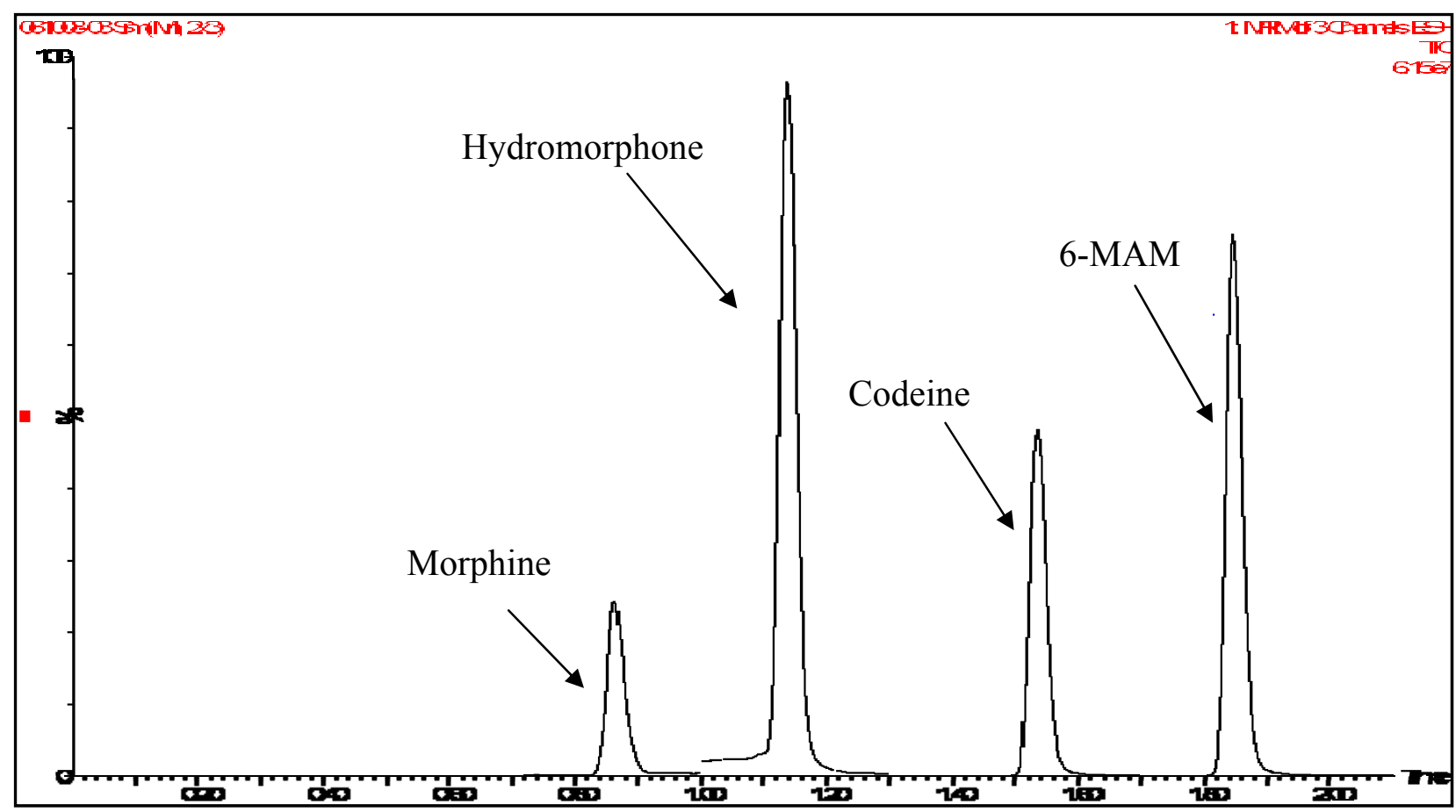

Figure 24: TIC from the UPLC separation of morphine, hydromorphone, codeine, and 6monoacetylmorphine (6-MAM).

\subsubsection{Mass Spectrometry}

Mass spectral conditions were as follows: capillary voltage $0.40 \mathrm{kV}$, cone voltage $48 \mathrm{~V}$, extractor voltage $3.1 \mathrm{~V}$, RF lens voltage $0.1 \mathrm{~V}$. The source temperature was $150^{\circ} \mathrm{C}$ while the desolvation temperature was set at $450^{\circ} \mathrm{C}$. Cone gas was set at a flow of $50 \mathrm{~L} / \mathrm{Hr}$ while the desolvation gas flow was $900 \mathrm{~L} / \mathrm{Hr}$. The collision gas flow was set to $0.18 \mathrm{~mL} / \mathrm{min}$. Nitrogen (99.995\% purity) was used as the desolvation gas, and ultra-pure argon (99.999\% purity) was used as the collision gas. Table 25 reports the mass transitions, dwell times, cone voltages, and collision energies for each of the analytes and their deuterated internal standards. 
Table 25: MS/MS parameters used for each analyte and their deuterated internal standards.

\begin{tabular}{|c|c|c|c|c|c|}
\hline Compound & Mass & Purpose & Cone (V) & Collision (V) & Dwell (secs) \\
\hline Codeine & $300.01>164.90$ & Quantifying ion & 45 & 40 & 0.02 \\
\hline Codeine & $300.01>215.00$ & Qualifying ion & 45 & 22 & 0.02 \\
\hline Codeine-d3 & $303.03>215.00$ & Quantifying ion & 50 & 26 & 0.02 \\
\hline Morphine & $285.97>164.90$ & Quantifying ion & 50 & 36 & 0.02 \\
\hline Morphine & $285.97>152.50$ & Qualifying ion & 50 & 46 & 0.02 \\
\hline Morphine-d3 & $288.89>200.90$ & Quantifying ion & 50 & 26 & 0.02 \\
\hline Hydromorphone & $285.98>184.90$ & Quantifying ion & 55 & 28 & 0.02 \\
\hline Hydromorphone & $285.98>156.80$ & Qualifying ion & 55 & 40 & 0.02 \\
\hline Hydromorphone-d3 & $288.97>184.90$ & Quantifying ion & 50 & 30 & 0.02 \\
\hline 6-monoacetlymorphine & $328.01>164.90$ & Quantifying ion & 55 & 36 & 0.02 \\
\hline 6-monoacetlymorphine & $328.01>210.90$ & Qualifying ion & 55 & 26 & 0.02 \\
\hline 6-monoacetlymorphine-d6 & $334.10>164.90$ & Quantifying ion & 55 & 36 & 0.02 \\
\hline
\end{tabular}


Figure 25 illustrates the fragmentation pathways for codeine, morphine, hydromorphone, and 6monoacetylmorphine under ESI conditions and the resulting product ions used for analyte detection.

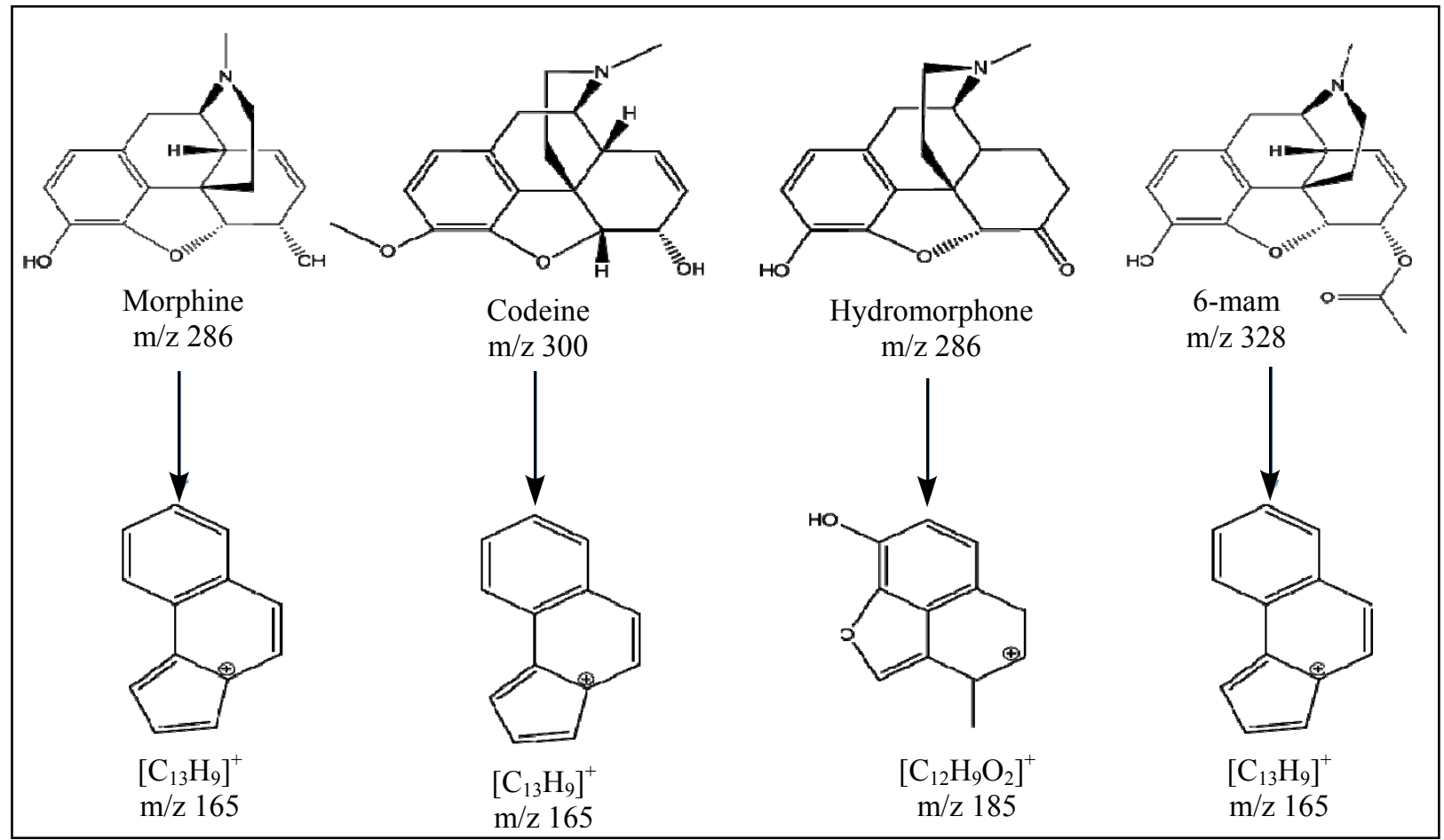

Figure 25: Fragmentation pathways for morphine, codeine, hydromorphone, and 6monoacetylmorphine during tandem mass spectrometry experiments[244].

\subsection{Results and Discussion}

\subsubsection{Method Validation}

Initial equivalence studies indicated that calibration curves prepared in certified drug-free blood were appropriate for the quantitation of codeine, morphine, hydromorphone, and 6monoacetylmorphine quality control standards prepared in negative meconium. Accurate quantification of QCs and a high degree of correlation between the calibration curves prepared in blood and those prepared in meconium indicate that curves prepared in drug-free blood will enable accurate quantitations of analytes in authentic meconium specimens (Table 26-29). Relative errors were calculated for all QC standards following quantitation with curves 
constructed in blood and those constructed in meconium. Relative errors did not exceed $9 \%$ for LQC (10 $\mathrm{ng} / \mathrm{mL})$ standards while the relative error associated with quantitating HQC (500 $\mathrm{ng} / \mathrm{mL}$ ) standards did not exceed $2.5 \%$ indicating a high degree of correlation for all four analytes. Calibration curves constructed in certified drug-free blood were employed for the remainder of validation experiments.

Table 26: Codeine equivalence studies using quantitative values obtained from calibration curves constructed in meconium (MC) and blood (BC).

\begin{tabular}{|c|c|c|c|}
\hline Codeine $\mathbf{S t d}$ & ${ }^{*} \mathbf{B C}(\mathbf{n g} / \mathbf{m L})$ & ${ }^{*} \mathbf{M C}(\mathbf{n g} / \mathbf{m L})$ & $\mathbf{\% R E}$ \\
\hline $\mathrm{LQC}$ & 10.6 & 9.8 & $8.2 \%$ \\
\hline $\mathrm{HQC}$ & 506.8 & 507.2 & $0.1 \%$ \\
\hline $\mathrm{NEG}(\mathrm{B})$ & 0.1 & 0 & $\mathrm{~N} / \mathrm{A}$ \\
\hline $\mathrm{NEG}(\mathrm{M})$ & 0.1 & 0 & $\mathrm{~N} / \mathrm{A}$ \\
\hline
\end{tabular}

*Reported concentrations are mean values of 5 replicate analyses.

Table 27: Morphine equivalence studies using quantitative values obtained from calibration curves constructed in meconium (MC) and blood (BC).

\begin{tabular}{|c|c|c|c|}
\hline Morphine Std & $* \mathbf{B C}(\mathbf{n g} / \mathbf{m L})$ & $* \mathbf{M C}(\mathbf{n g} / \mathbf{m L})$ & $\mathbf{\% R E}$ \\
\hline LQC & 9.0 & 9.2 & $2.2 \%$ \\
\hline HQC & 504.7 & 517.2 & $2.4 \%$ \\
\hline NEG(B) & 0 & 0 & N/A \\
\hline NEG(M) & 0 & 0 & N/A \\
\hline
\end{tabular}

*Reported concentrations are mean values of 5 replicate analyses.

Table 28: Hydromorphone equivalence studies using quantitative values obtained from calibration curves constructed in meconium (MC) and blood (BC).

\begin{tabular}{|c|c|c|c|}
\hline Hydromorphone Std & ${ }^{*} \mathbf{B C}(\mathbf{n g} / \mathbf{m L})$ & $* \mathbf{M C}(\mathbf{n g} / \mathbf{m L})$ & $\mathbf{\% R E}$ \\
\hline LQC & 9.5 & 9.3 & $2.2 \%$ \\
\hline HQC & 492.9 & 490.1 & $0.6 \%$ \\
\hline NEG(B) & 0 & 0 & N/A \\
\hline NEG(M) & 0 & 0 & N/A \\
\hline
\end{tabular}

*Reported concentrations are mean values of 5 replicate analyses. 
Table 29: 6-monoacetylmorphine (6-MAM) equivalence studies using quantitative values obtained from calibration curves constructed in meconium (MC) and blood (BC).

\begin{tabular}{|c|c|c|c|}
\hline 6-monoacetylmorphine Std & $* \mathbf{B C} \mathbf{( n g} / \mathbf{m L})$ & $* \mathbf{M C}(\mathbf{n g} / \mathbf{m L})$ & $\mathbf{\% R E}$ \\
\hline LQC & 9.8 & 9.1 & $7.7 \%$ \\
\hline HQC & 491.0 & 482.4 & $1.8 \%$ \\
\hline NEG(B) & 0 & 0 & N/A \\
\hline NEG(M) & 0 & 0 & N/A \\
\hline
\end{tabular}

*Reported concentrations are mean values of 5 replicate analyses.

The analytical methodology was deemed selective following the analysis of blank meconium specimens from five different sources and the accurate quantitation of five standards prepared at the LLOQ which had been spiked with various xenobiotics commonly encountered in forensic toxicology (Table 30).

Table 30: Exogenous compounds included in selectivity quality control standards.

\begin{tabular}{|c|l|}
\hline Standard & \multicolumn{1}{c|}{ Contents } \\
\hline Selectivity QC 2 & $\begin{array}{l}\text { Amitriptyline, bupropion, clomipramine, desipramine, doxepin, fluoxetine, imipramine, } \\
\text { norclomipramine, nordoxepin, norfluoxetine, norsertraline, nortriptyline, norvenlafaxine, } \\
\text { paroxetine, sertraline, venlafaxine }\end{array}$ \\
\hline Selectivity QC 3 & $\begin{array}{l}\text { Alprazolam, chlorpheniramine, citalopram, clonazepam, cyclobenzaprine, dextromethorphan, } \\
\text { duloxetine, fentanyl, flunitrazepam, haloperidol, mirtazapine, olanzapine, strychnine, zolpidem }\end{array}$ \\
\hline Selectivity QC 4 & $\begin{array}{l}\text { Chlordiazepoxide, chlorpromazine, clozapine, diazepam, lorazepam, midazolam, norclozapine, } \\
\text { nordiazepam, oxazepam, PMA, quetiapine, temazepam }\end{array}$ \\
\hline Selectivity QC 5 & $\begin{array}{l}\text { Amphetamine, benzoylecgonine, cocaethylene, cocaine, EDDP, ephedrine, MDMA, } \\
\text { methadone, methamphetamine, norpropoxyphene, nortramadol, PCP, propoxyphene, tramadol }\end{array}$ \\
\hline Selectivity QC 6 & $\begin{array}{l}\text { Alfentanil, diphenhydramine, doxylamine, meperidine, normeperidine, orphenadrine, } \\
\text { promethazine, propranolol, trazodone, trimipramine, verapamil }\end{array}$ \\
\hline
\end{tabular}

Blank meconium matrices failed to generate a response for any of the four analytes investigated indicating that the method is sufficiently selective to eliminate the possibility of matrix interferences generating false positives (Table 31-34). Accurate quantitation of QC standards prepared at the LLOQ which had been spiked with various exogenous interferences commonly encountered in forensic specimens indicated that the methodology is selective for the analytes of 
interest (Table 31-34). Quantitation accuracies ranged from $94 \%$ to $100 \%$ for codeine, $91 \%$ to $96 \%$ for morphine, $94 \%$ to $97 \%$ for hydromorphone, and $92 \%$ to $99 \%$ for 6 -

monoacetylmorphine. Quantitation accuracy at the LLOQ indicates a high degree of selectivity in the presence of exogenous interferences.

Table 31: Codeine selectivity studies using blank meconium from five different sources and blank meconium specimens spiked with various exogenous interferences.

\begin{tabular}{|c|c|c|c|c|}
\hline Codeine Std & Std Conc & $\begin{array}{c}\text { *Measured } \\
\text { Conc }\end{array}$ & Acceptable range & Pass/Fail \\
\hline Blank samples & 0 & 0 & $<10 \mathrm{ng} / \mathrm{mL}$ & Pass \\
\hline Spiked samples & 10 & 10.0 & $10+/-20 \%$ & Pass \\
\hline
\end{tabular}

*Reported concentrations are mean values of 5 replicate analyses.

Table 32: Morphine selectivity studies using blank meconium from five different sources and blank meconium specimens spiked with various exogenous interferences.

\begin{tabular}{|c|c|c|c|c|}
\hline Morphine Std & Std Conc & $\begin{array}{c}* \text { Measured } \\
\text { Conc }\end{array}$ & Acceptable range & Pass/Fail \\
\hline Blank samples & 0 & 0 & $<10 \mathrm{ng} / \mathrm{mL}$ & Pass \\
\hline Spiked samples & 10 & 9.3 & $10+/-20 \%$ & Pass \\
\hline
\end{tabular}

*Reported concentrations are mean values of 5 replicate analyses.

Table 33: Hydromorphone selectivity studies using blank meconium from five different sources and blank meconium specimens spiked with various exogenous interferences.

\begin{tabular}{|c|c|c|c|c|}
\hline Hydromorphone Std & Std Conc & $\begin{array}{c}\text { *Measured } \\
\text { Conc }\end{array}$ & Acceptable range & Pass/Fail \\
\hline Blank samples & 0 & 0 & $<10 \mathrm{ng} / \mathrm{mL}$ & Pass \\
\hline Spiked samples & 10 & 9.6 & $10+/-20 \%$ & Pass \\
\hline
\end{tabular}

*Reported concentrations are mean values of 5 replicate analyses.

Table 34: 6-monoacetylmorphine selectivity studies using blank meconium from five different sources and blank meconium specimens spiked with various exogenous interferences.

\begin{tabular}{|c|c|c|c|c|}
\hline 6-monoacetylmorphine Std & Std Conc & Measured Conc & Acceptable range & Pass/Fail \\
\hline Blank samples & 0 & 0 & $<10 \mathrm{ng} / \mathrm{mL}$ & Pass \\
\hline Spiked samples & 10 & 10.1 & $10+/-20 \%$ & Pass \\
\hline
\end{tabular}

*Reported concentrations are mean values of 5 replicate analyses.

Accuracy of the methodology was investigated by analyzing five replicates over three concentrations spanning the calibration range. Accuracy was investigated at concentrations of 
500,50 , and $10 \mathrm{ng} / \mathrm{mL}$ for all analytes. Mean values of $487.1 \mathrm{ng} / \mathrm{mL}, 50.2 \mathrm{ng} / \mathrm{mL}$, and $9.7 \mathrm{ng} / \mathrm{mL}$ reflecting accuracies of $97.4 \%, 99.6 \%$, and $97 \%$ were obtained for codeine (Table 35), while analysis of morphine standards yielded mean values of $478.6 \mathrm{ng} / \mathrm{mL}, 46.5 \mathrm{ng} / \mathrm{mL}$, and $9.9 \mathrm{ng} / \mathrm{mL}$ corresponding to accuracies of $95.7 \%, 93 \%$, and 99\% (Table 36). High accuracies were also observed through the analysis of hydromorphone standards which produced mean values of $493.9 \mathrm{ng} / \mathrm{mL}, 49.8 \mathrm{ng} / \mathrm{mL}$, and $9.8 \mathrm{ng} / \mathrm{mL}$ corresponding to accuracies of $98.8 \%, 99.6 \%$, and 98\% (Table 37). Analysis of 6-monoacetlymorphine QC standards prepared at concentrations of 500,50 , and $10 \mathrm{ng} / \mathrm{mL}$ yielded mean values of $515.7 \mathrm{ng} / \mathrm{mL}, 52.0 \mathrm{ng} / \mathrm{mL}$, and $9.8 \mathrm{ng} / \mathrm{mL}$ corresponding to accuracies of $96.9 \%, 96 \%$, and 98\% (Table 38). Quantitation accuracy over the calibration range was greater than $93 \%$ for all four analytes.

Table 35: Codeine accuracy over three concentrations spanning the calibration range.

\begin{tabular}{|c|c|c|c|}
\hline Codeine Std & Std Conc & *Measured value & \%RE \\
\hline High point & 500 & 487.1 & $2.6 \%$ \\
\hline Midpoint & 50 & 50.2 & $0.4 \%$ \\
\hline LLOQ & 10 & 9.7 & $3.0 \%$ \\
\hline
\end{tabular}

*Reported concentrations are mean values of 5 replicate analyses.

Table 36: Morphine accuracy over three concentrations spanning the calibration range.

\begin{tabular}{|c|c|c|c|}
\hline Morphine Std & Std Conc & $*$ Measured value & \%RE \\
\hline High point & 500 & 478.6 & $4.3 \%$ \\
\hline Midpoint & 50 & 46.5 & $7.0 \%$ \\
\hline LLOQ & 10 & 9.9 & $1.0 \%$ \\
\hline
\end{tabular}

*Reported concentrations are mean values of 5 replicate analyses.

Table 37: Hydromorphone accuracy over three concentrations spanning the calibration range.

\begin{tabular}{|c|c|c|c|}
\hline Hydromorphone Std & Std Conc & *Measured value & \%RE \\
\hline High point & 500 & 493.9 & $1.2 \%$ \\
\hline Midpoint & 50 & 49.8 & $0.4 \%$ \\
\hline LLOQ & 10 & 9.8 & $2.0 \%$ \\
\hline
\end{tabular}

*Reported concentrations are mean values of 5 replicate analyses. 
Table 38: 6-monoacetylmorphine accuracy over three concentrations spanning the calibration range.

\begin{tabular}{|c|c|c|c|}
\hline 6-monoacetylmorphine Std & Std Conc & $*$ Measured value & \%RE \\
\hline High point & 500 & 515.7 & $3.1 \%$ \\
\hline Midpoint & 50 & 52.0 & $4.0 \%$ \\
\hline LLOQ & 10 & 9.8 & $2.0 \%$ \\
\hline
\end{tabular}

*Reported concentrations are mean values of 5 replicate analyses.

Intra- and inter-batch precision was investigated during method validation. Five replicates prepared at three different concentrations spanning the calibration range were analyzed and mean values were calculated for each concentration to determine the precision associated with the methodology. Quality control standards were prepared in negative meconium at concentrations of 10,50 , and $500 \mathrm{ng} / \mathrm{mL}$ for each analyte. Both intra- and inter-batch precision studies indicated a high degree of method precision over the investigated concentration range with intra-batch CVs ranging from $1.4-9.5 \%$ with a mean value of $2.9 \%$ for codeine (Table 39), while intra-batch morphine studies produced CVs ranging from $1.2-10.7 \%$ with a mean value of 3.1\% (Table 40). Analysis of hydromorphone QCs produced intra-batch CVs ranging from 1.4$7.6 \%$ with a mean value of $3.3 \%$ (Table 41 ), while 6-monoacetylmorphine standards yielded CVs ranging from $1.3-10 \%$ with a mean value of 3.3\% (Table 42). Method precision was high over the entire calibration range including the LLOQ where individual CVs did not exceed 10.7\% (Tables 39-42). Inter-batch precision calculations were performed to assess method precision over a four day period. CVs were calculated based on four-day mean values for QCs at concentrations of 500,50 , and $5 \mathrm{ng} / \mathrm{mL}$ and produced values of $3.3 \%, 4.5$, and $4.7 \%$ respectively for codeine, $3.1 \%, 4.0 \%$, and $4.7 \%$ for morphine, $3.1 \%, 2.3 \%$, and $6.1 \%$ for hydromorphone, and $1.4 \%, 0.5 \%$, and $5.2 \%$ for 6-monoacetlymorphine (Tables $39-42$ ). 
Table 39: Intra- and inter-batch precision for codeine.

\begin{tabular}{|c|c|c|c|c|c|c|}
\hline Codeine Std & *Day 1 value & *Day 2 value & *Day 3 value & *Day 4 value & 4 day mean & 4 day CV \\
\hline Highpoint & 487.1 & 505.8 & 507.6 & 527.8 & 507.1 & $3.3 \%$ \\
\hline CV & $1.4 \%$ & $1.9 \%$ & $1.7 \%$ & $1.8 \%$ & & \\
\hline Midpoint & 50.2 & 53.3 & 50.0 & 54.7 & 52.0 & $4.5 \%$ \\
\hline CV & $1.6 \%$ & $2.6 \%$ & $1.5 \%$ & $2.9 \%$ & & \\
\hline LLOQ & 9.7 & 10.5 & 9.6 & 10.5 & 10.1 & $4.7 \%$ \\
\hline CV & $4.9 \%$ & $2.3 \%$ & $2.6 \%$ & $9.5 \%$ & & \\
\hline
\end{tabular}

*Reported concentrations are mean values of 5 replicate analyses.

Table 40: Intra- and inter-batch precision for morphine.

\begin{tabular}{|c|c|c|c|c|c|c|}
\hline Morphine Std & *Day 1 value & *Day 2 value & *Day 3 value & *Day 4 value & 4 day mean & 4 day CV \\
\hline Highpoint & 478.6 & 499.9 & 466.3 & 494.0 & 484.7 & $3.1 \%$ \\
\hline CV & $1.4 \%$ & $1.8 \%$ & $1.5 \%$ & $2.7 \%$ & & \\
\hline Midpoint & 46.5 & 45.5 & 45.0 & 49.2 & 46.6 & $4.0 \%$ \\
\hline CV & $2.7 \%$ & $2.6 \%$ & $3.0 \%$ & $5.5 \%$ & & \\
\hline LLOQ & 9.9 & 9.2 & 9.1 & 8.9 & 9.3 & $4.7 \%$ \\
\hline CV & $1.2 \%$ & $2.6 \%$ & $1.6 \%$ & $10.7 \%$ & & \\
\hline
\end{tabular}

*Reported concentrations are mean values of 5 replicate analyses.

Table 41: Intra- and inter-batch precision for hydromorphone.

\begin{tabular}{|c|c|c|c|c|c|c|}
\hline Hydromorphone & *Day 1 value & *Day 2 value & *Day 3 value & *Day 4 value & 4 day mean & 4 day CV \\
\hline Highpoint & 493.9 & 499.3 & 508.2 & 529.2 & 507.7 & $3.1 \%$ \\
\hline CV & $2.5 \%$ & $2.7 \%$ & $4.4 \%$ & $2.7 \%$ & & \\
\hline Midpoint & 49.8 & 48.6 & 47.7 & 50.2 & 49.1 & $2.3 \%$ \\
\hline CV & $2.0 \%$ & $1.4 \%$ & $3.4 \%$ & $2.7 \%$ & & \\
\hline LLOQ & 9.8 & 8.9 & 9.2 & 10.2 & 9.5 & $6.1 \%$ \\
\hline CV & $2.6 \%$ & $3.8 \%$ & $3.4 \%$ & $7.6 \%$ & & \\
\hline
\end{tabular}

*Reported concentrations are mean values of 5 replicate analyses.

Table 42: Intra- and inter-batch precision for 6-monoacetylmorphine (6-MAM).

\begin{tabular}{|c|c|c|c|c|c|c|}
\hline 6-MAM Std & *Day 1 value & *Day 2 value & *Day 3 value & *Day 4 value & 4 day mean & 4 day CV \\
\hline Highpoint & 515.7 & 525.9 & 528.0 & 534.0 & 525.9 & $1.4 \%$ \\
\hline CV & $1.3 \%$ & $3.0 \%$ & $2.2 \%$ & $2.9 \%$ & & \\
\hline Midpoint & 52.0 & 52.3 & 52.2 & 52.6 & 52.3 & $0.5 \%$ \\
\hline CV & $2.2 \%$ & $3.0 \%$ & $4.1 \%$ & $3.6 \%$ & & \\
\hline LLOQ & 9.8 & 10.6 & 10.5 & 9.6 & 10.1 & $5.2 \%$ \\
\hline CV & $1.9 \%$ & $2.4 \%$ & $2.6 \%$ & $10.0 \%$ & & \\
\hline
\end{tabular}

*Reported concentrations are mean values of 5 replicate analyses. 
Analyte recovery was investigated over three concentrations by comparing the detector response of analyte at three different concentrations which had been added to, and extracted from, the meconium matrix. Analyte recovery was investigated by comparing peak areas of extracted samples at concentrations of 10,50 , and $500 \mathrm{ng} / \mathrm{mL}$ with unextracted samples which represented $100 \%$ recovery. Mean analyte recoveries over the three concentrations investigated were $64.3 \%, 52.3 \%, 55.9 \%$, and $76.3 \%$ for codeine, morphine, hydromorphone, and 6 monacetylmorphine respectively. Mean recoveries from triplicate analysis at the three concentrations investigated ranged from $61-69 \%$ for codeine (Figure 26), 47-61\% for morphine (Figure 27), 48-64\% for hydromorphone (Figure 28), and 68-84\% for 6-monoacetylmorphine (Figure 29).

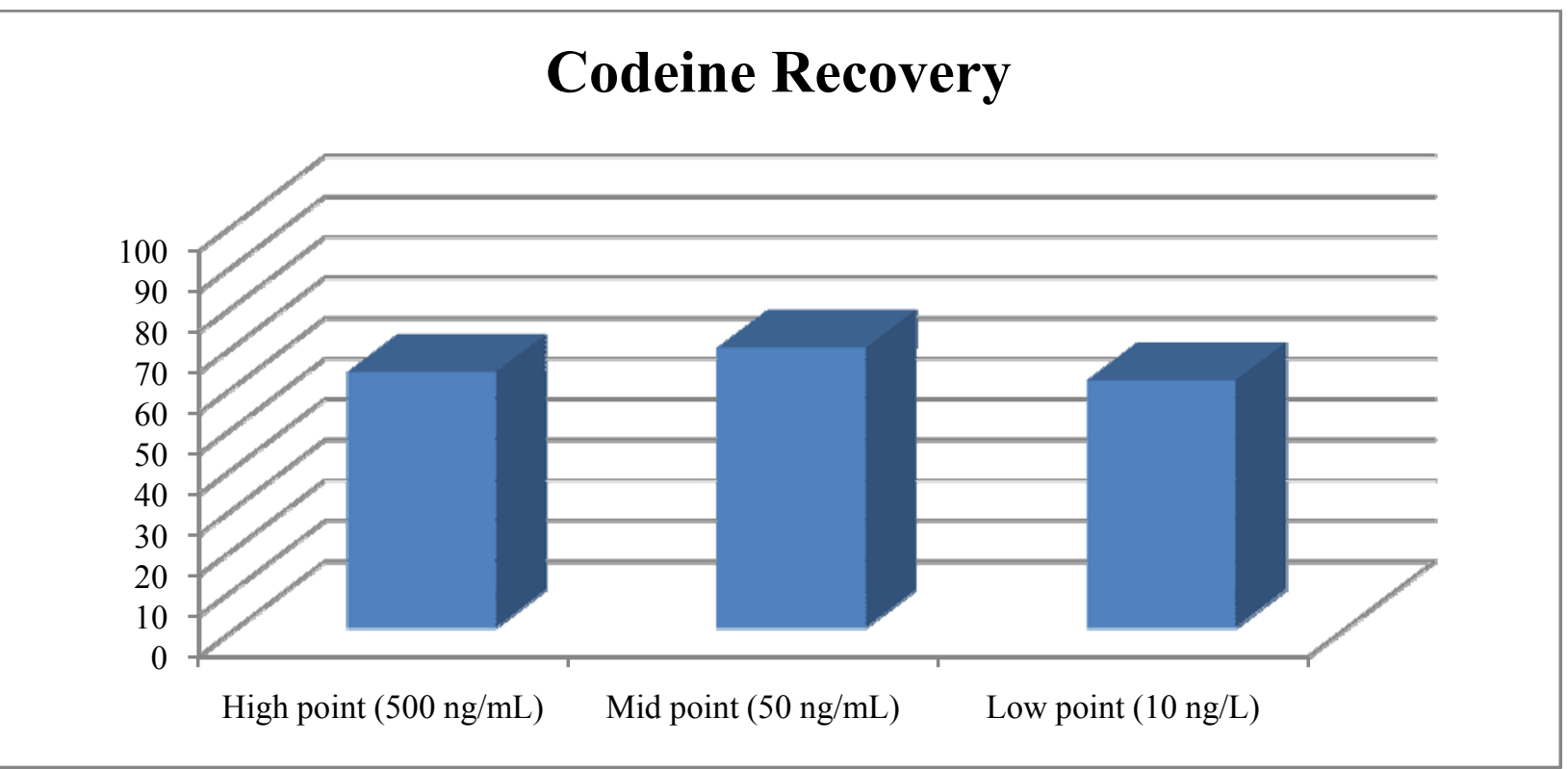

Figure 26: Codeine extraction recovery. 


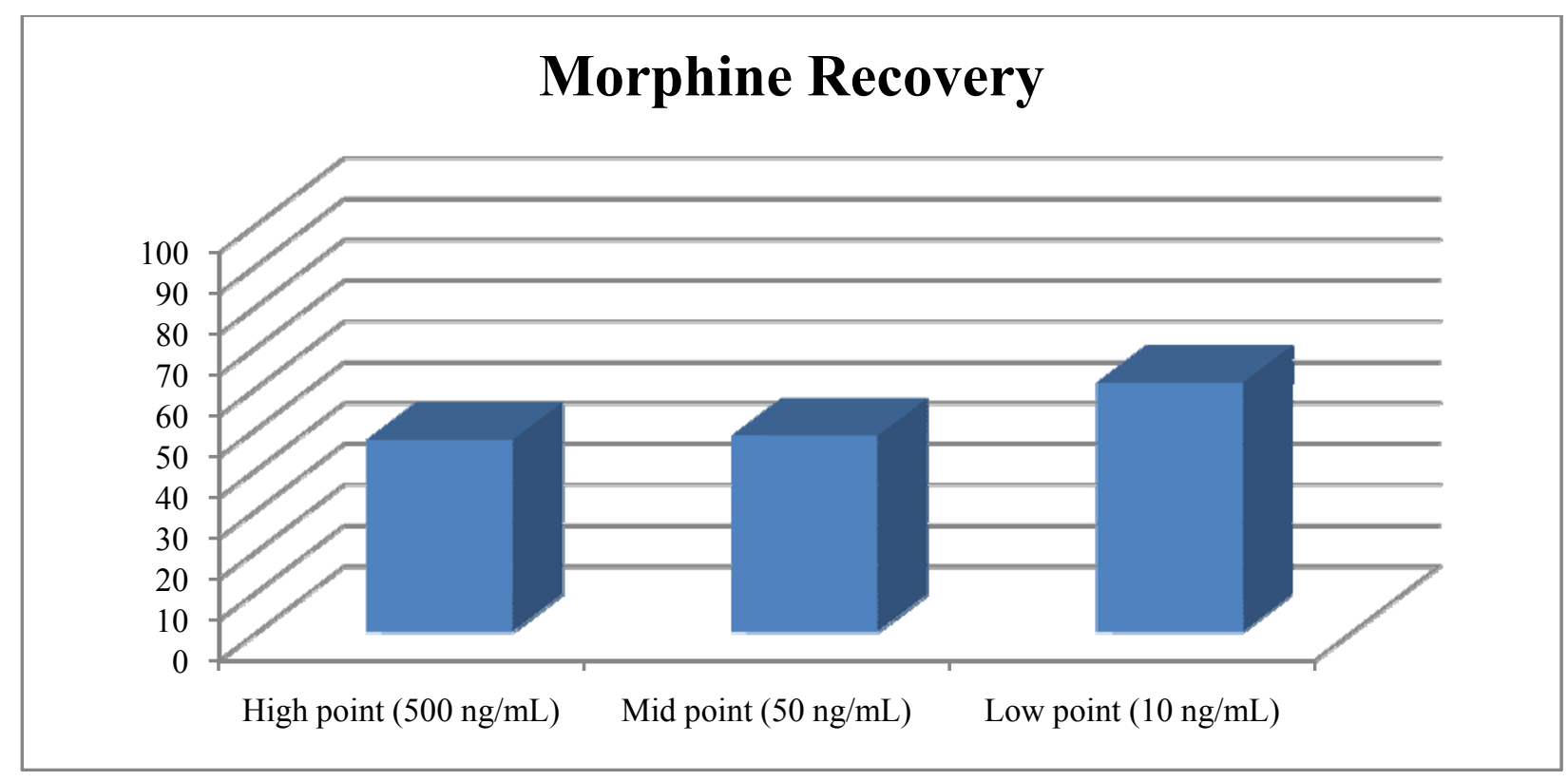

Figure 27: Morphine extraction recovery.

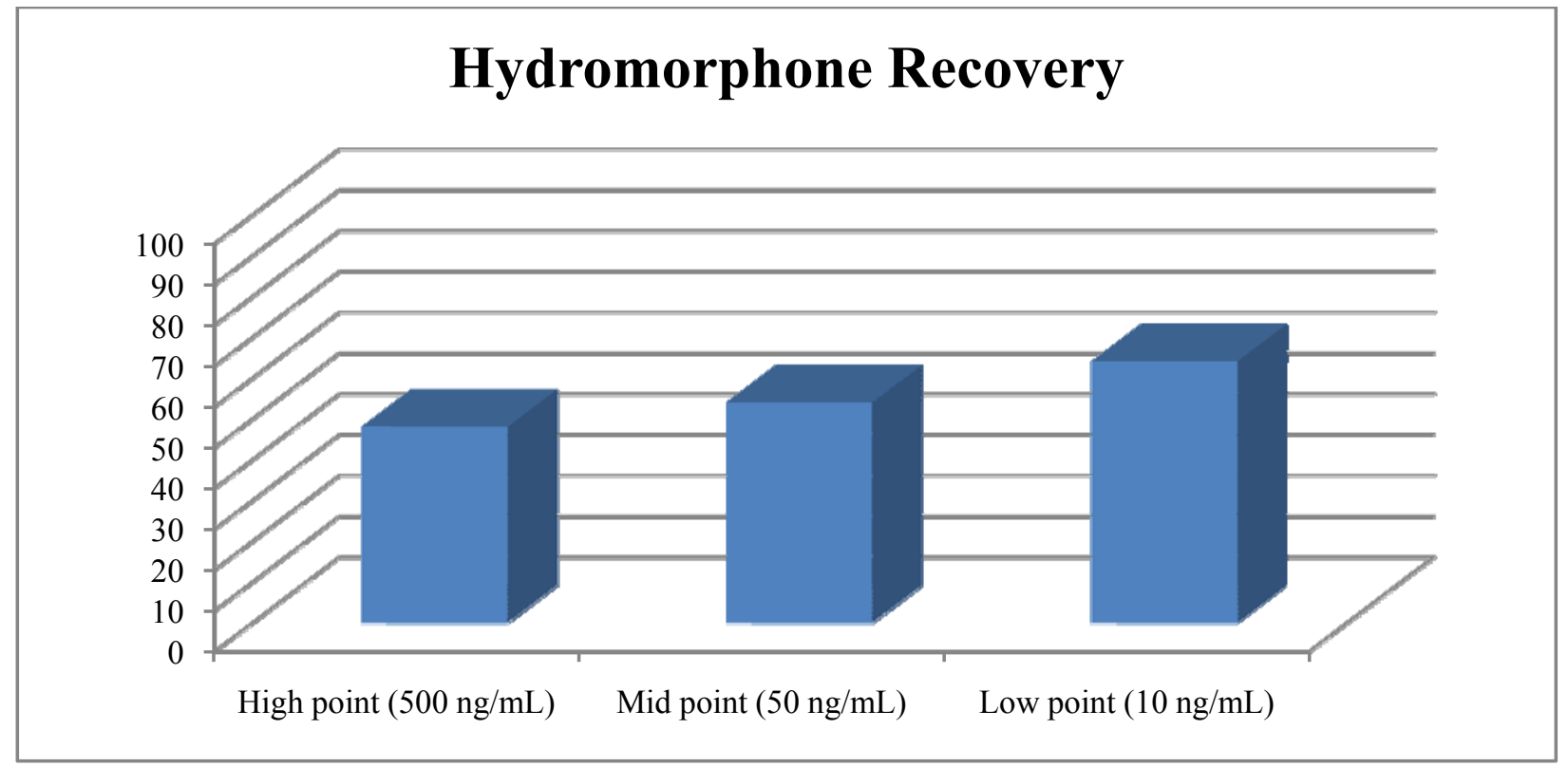

Figure 28: Hydromorphone extraction recovery. 


\section{6-monoacetylmorphine Recovery}

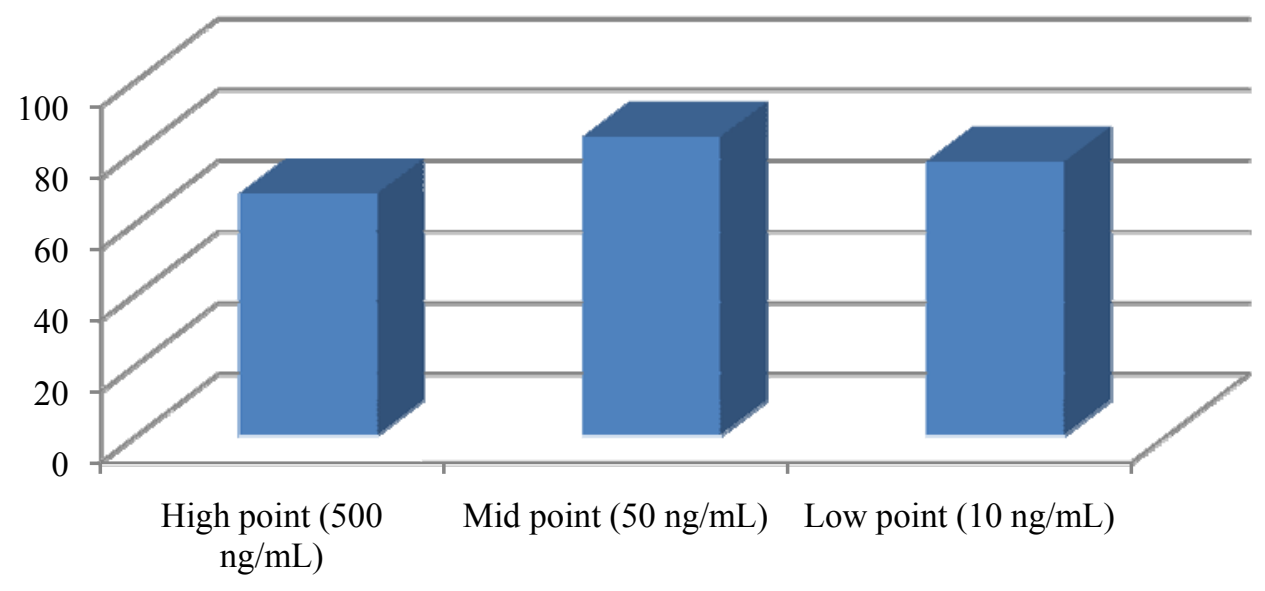

Figure 29: 6-MAM extraction recovery.

The limit of detection was $2.5 \mathrm{ng} / \mathrm{mL}$ for all four analytes corresponding to a concentration of 10 $\mathrm{ng} / \mathrm{g}$ in the original meconium specimen. Limits of detection were calculated based on acceptance criteria for retention times and ion ratios. At concentrations below $2.5 \mathrm{ng} / \mathrm{mL}$ unequivocal identification was not possible due to inaccurate ion ratios. Stability studies indicated that all four analytes were stable in the meconium matrix when stored long-term at $4^{\circ} \mathrm{C}$. High and low QC standards corresponding to the calibration range were subject to a 72 hour freeze-thaw cycle and analyzed following thawing on the third day. Quantitation accuracies during stability studies were greater than 97\%, and 94\% for codeine LQCs and HQCs respectively, greater than 98\%, and 92\% for morphine low and high QCs, greater than 98\%, and 99\% for hydromorphone low and high QCs and greater than $96 \%$, and $99 \%$ for $6-$ monoacetylmorphine low and high QCs respectively. Accurate quantitations during stability studies indicate that storage temperature and conditions are suitable for authentic meconium 
specimens and any accurate quantitation of the four analytes following thawing should be feasible.

\subsection{Conclusions}

Development, optimization and validation of a UPLC-ESI-MS/MS method allowed for the accurate, selective, precise, and sensitive determination of morphine, codeine, hydromorphone, and 6-monoacetylmorphine following supported liquid extraction from meconium specimens. Extraction of all four analytes from the meconium matrix using ISOLUTE HM-N supported liquid extraction columns significantly reduced sample preparation time while the rapid separations afforded by UPLC significantly reduced analysis time, allowing for increased sample throughput. The increased separation efficiency afforded by UPLC, combined with the inherent selectivity and sensitivity of tandem mass spectrometric detection is well suited to low level, multi-analyte determination in complex biological matrices such as meconium. Rapid UPLC separations combined with certain automated features of the Waters TQD instrument, significantly reduces method development and validation time, making the instrumental combination well suited to high throughput toxicology laboratories. Two MRM transitions were monitored for each analyte and quantifications were made using deuterated internal standards and seven point calibration curves. Detection limits for all four analytes was $2.5 \mathrm{ng} / \mathrm{mL}$ in the homogenized extract, and injection volumes of $5 \mu \mathrm{L}$ allowed for the linear quantification of analytes up to $500 \mathrm{ng} / \mathrm{mL}$. To the author's knowledge, this is the first validated UPLC-MS/MS methodology for the quantification of morphine, codeine, hydromorphone, and 6monoacetylmorphine in meconium specimens. 


\section{Chapter 7: Simultaneous Quantification of Amphetamine and Methamphetamine in Meconium Using ISOLUTE ${ }^{\circledR}$ HM-N Supported Liquid Extraction Columns and UPLC-MS/MS}

\subsection{Abstract}

A procedure for the rapid extraction and quantification of amphetamine and methamphetamine from meconium using ISOLUTE HM-N supported liquid extraction columns and ultra performance liquid chromatography coupled with tandem mass spectrometry (UPLCMS/MS) is described. Due to the matrix complexity of meconium samples, extraction and sample preparation prior to instrumental analysis can prove difficult and time consuming. The present study introduces a novel sample preparation technique for the simultaneous quantification of amphetamine and methamphetamine in meconium using UPLC-MS/MS.

Ultra performance liquid chromatography (UPLC) is an emerging analytical technique which draws upon the principles of chromatography to run separations at higher flow rates for increased speed, while simultaneously achieving superior resolution and sensitivity.

Extraction of both analytes was achieved using ISOLUTE HM-N supported liquid extraction columns containing a modified form of diatomaceous earth. Subsequent separation and quantification using ultra UPLC-MS/MS was achieved in less than 3 minutes. Limits of detection for amphetamine and methamphetamine were $4 \mathrm{ng} / \mathrm{g}$ and $1000 \mathrm{pg} / \mathrm{g}$ respectively. The lower limit of quantitation (LLOQ) was $20 \mathrm{ng} / \mathrm{g}$. The methodology exhibited high intra run precision with $\mathrm{CV}$ values ranging from $1-9 \%$ for amphetamine and $1-6 \%$ for methamphetamine. Inter run precision experiments produced CV values ranging from 3-7\% for amphetamine and 1$6 \%$ for methamphetamine. 
The reported methodology proved suitable for the accurate quantification of amphetamine and methamphetamine in meconium samples and greatly reduced sample preparation time normally required for traditional solid phase extraction. Development and validation of such analytical methodologies will prove beneficial for the identification of prenatal substance abuse which.

\subsection{Introduction}

Due to the increasing abuse and synthesis of amphetamine like stimulants, there is a desire among analytical chemists for sensitive methodologies capable of detecting low levels of these drugs in meconium. Such methodologies would aid in further understanding the effects of fetal exposure on newborns. Clefting, cardiac anomalies, and fetal growth reduction deficits have all been seen in infants exposed to amphetamines during pregnancy [245]. Animal studies involving prenatal exposure to amphetamines have allowed for the same observations and methamphetamine has been shown to cross the placenta within thirty seconds of intraperitoneal injection [246]. Methamphetamine is the most widely abused amphetamine and animal studies observed increased maternal and offspring mortality, retinal eye defects, cleft palate, rib malformations, decreased rate of physical growth, and delayed motor development associated with prenatal methamphetamine exposure [247-250]. Although peak concentrations are lower on the fetal side, slower elimination of the amphetamines means that the fetus is subject to prolonged exposure which can significantly impact neonatal health and development [246].

In the first large scale investigation into the prevalence of methamphetamine use during pregnancy in areas of the United States where methamphetamine is a notable concern, it was found that $5.2 \%$ of the 1632 subject mothers used methamphetamine at some point during their pregnancy [251]. The authors concluded that the methamphetamine exposed group was 3.5 times 
more likely to be small for gestational age than the unexposed group [252]. Prenatal exposure to amphetamines has also been associated with behavioral problems, cranial abnormalities, increased incidence of premature delivery, placental abruption, increased rates of fetal distress, biliary atresia, and decreased growth parameters (weight, head circumference, length)[253, 254]. Table 43 provides the chemical structures of methamphetamine and amphetamine along with certain physicochemical properties such as molecular weights, and pka's.

Table 43: Chemical structures, molecular weights, molecular formulas and $\mathrm{pKa}$ 's of amphetamine and methamphetamine.

\begin{tabular}{|l|l|l|l|}
\hline \multicolumn{2}{|c|}{ Amphetamine } & Methamphetamine \\
\hline Chemical structure & Molecular weight \\
\hline Molecular weight & $135.21 \mathrm{~g} / \mathrm{mol}$ & Molecular formula & $149.23 \mathrm{~g} / \mathrm{mol}$ \\
\hline Molecular formula & $\mathrm{C}_{9} \mathrm{H}_{13} \mathrm{~N}$ & $\mathrm{pKa}$ & 10.1 \\
\hline pKa & 9.80 & $\mathrm{C}_{10} \mathrm{H}_{15} \mathrm{~N}$ \\
\hline
\end{tabular}

\subsection{Mechanism of Action}

The amphetamines are similar to cocaine in their ability to modify the actions and levels of catecholamines. Amphetamines acts to stimulate the sympathomimetic nervous system both centrally and peripherally. This again is achieved through increasing levels of dopamine and norepinephrine, however, the mechanism by which the amphetamines achieve this differs slightly from other stimulants such as cocaine. Amphetamines are chemically similar to dopamine and norepinephrine allowing them to enter the presynaptic terminal assisted by protein molecules that would normally transport dopamine and norepinephrine back into the nerve 
terminal from the synaptic cleft. Once in the presynaptic terminal, amphetamines acts to release dopamine and norepinephrine from vesicles resulting in increased levels of free catecholamines in the nerve ending. Amphetamines also inhibit monoamine oxidase (MAO), an enzyme responsible for the deactivation of free catecholamines in the presynaptic terminal. As a result, excess levels of dopamine and norepinephrine are transported out of the presynaptic terminal and into the synapse where they produce feelings of pleasure and euphoria.

\subsection{Effect of Prenatal Exposure}

Clefting, cardiac anomalies, fetal growth reduction deficits have all been seen in infants exposed to amphetamines during pregnancy, increased maternal and offspring mortality, retinal eye defects, cleft palate, rib malformations, decreased rate of physical growth, and delayed motor development have all been associated with prenatal exposure to amphetamines. Although peak concentrations are lower on the fetal side, slower elimination of the amphetamines means that the fetus is subject to prolonged exposure which can significantly impact neonatal health and development [245-250].

\subsection{History of Amphetamine Analysis in Meconium (Literature Review)}

Meconium has become the specimen of choice for the detection of prenatal exposure to several drugs of abuse $[255,256]$. There are several reasons for this, including the relatively simple and non-invasive procedure used to collect meconium samples, making it more successful than urine collection [70]. Meconium analysis also extends the window of drug detection to approximately the last 20 weeks of gestation as well as extending the window for specimen collection, as it is not fully evacuated until 125 hours post-natally [213, 257, 258]. 
Due to the complexity of the meconium matrix, analysis can prove difficult as sample preparation may require additional laborious steps to efficiently extract the desired analytes from the non-homogenous sample [259]. Ostrea et al. [260] employed a two stage extraction procedure for the detection of illicit drugs and other xenobiotics in newborn infants. Such procedures involve an initial liquid extraction from the meconium after which the organic layer is evaporated and reconstituted in phosphate buffer in preparation for solid-phase extraction. Conventional SPE columns require multi-step conditioning and subsequent aspiration before the sample can be introduced onto the column. Most SPE extraction procedures involve 2-3 sequential washes before analytes are eluted with a suitable solvent made fresh daily. The combination of a two stage extraction involving a multi wash SPE procedure with the need to prepare elution solvents daily can prove very laborious in high throughput laboratories.

ElSohly et al [243] achieved limits of detection of $50 \mathrm{ng} / \mathrm{g}$ for amphetamine and methamphetamine employing a multi-step liquid extraction procedure and GC/MS. Additional sample cleanup was achieved by incorporating a back extraction for the purpose of eliminating neutral molecules present in the matrix. Researchers at the National Institute on Drug Abuse (NIDA) recently developed the first reported tandem mass spectrometry method for the detection of 10 amphetamine-, methamphetamine- and 3,4-methylenedioxymethamphetamine-related (MDMA) analytes in human meconium. Specimens were homogenized and subject to solid phase extraction prior to chromatographic separation and mass spectrometric detection. The authors employed a triple quadrupole mass spectrometer equipped with an APCI source. Selective determination of the 10 analytes was achieved by monitoring two specific MRM transitions. The enhanced sensitivity of LC-MS/MS analysis relative to more traditional GC/MS analysis allowed for lower limits of quantitation in the range of 1.25 to $40 \mathrm{ng} / \mathrm{ng}$ while upper 
limits of quantitation were set at 10,000 ng/g. Successful detection of phydroxymethamphetamine, norephedrine and 4-hydroxy-3-methoxymethamphetamine in the meconium of a methamphetamine-exposed neonate was reported for the first time[93]. While such methodologies have allowed for the selective determination of amphetamines in meconium, sample preparation remains a time costly and limiting factor in the analysis of meconium. Laborious solid phase extractions following homogenization and initial liquid-liquid extractions can prove very time inefficient and greatly affect the turnaround time of high throughput toxicology laboratories.

The aim of the present study was to develop a time and cost effective methodology for the preparation of meconium samples which would allow for the rapid and simultaneous quantification of amphetamine and methamphetamine in meconium. In this report, we describe the first application of supported liquid extraction columns for the preparation of meconium specimens prior to analysis and quantification by UPLC-MS/MS. ISOLUTE HM-N supported

liquid extraction columns require no column conditioning and once the sample is introduced onto the column, elution is achieved with two washes of ethyl acetate. This is the first report of the use of UPLC-MS/MS for the determination of amphetamine and methamphetamine in meconium.

\subsection{Experimental}

\subsubsection{Chemicals and reagents}

Amphetamine, methamphetamine, amphetamine- $\mathrm{d}_{6}$, and methamphetamine- $\mathrm{d}_{9}$ standards $\left(1 \mathrm{mg} / \mathrm{mL}\right.$ in methanol) were obtained from Cerilliant (Round Rock, TX). ISOLUTE ${ }^{\circledR}$ HM-N 
supported liquid-liquid extraction columns were purchased from Biotage (Charlottesville, VA). All solvents were HPLC grade and obtained from Fisher Scientific (Pittsburgh PA).

\subsubsection{Calibration Curve Matrix}

Meconium specimens which had previously screened negative for amphetamines using a $50 \mathrm{ng} / \mathrm{g}$ cutoff at AIT laboratories (Indianapolis, IN) were collected and spiked with both amphetamine and methamphetamine to give concentrations of $10 \mathrm{ng} / \mathrm{mL}(\mathrm{n}=5)$ and $500 \mathrm{ng} / \mathrm{mL}$ $(n=5)$. Spiked meconium was then quantified using a calibration curve constructed in negative meconium and a calibration curve constructed in certified drug free whole blood. Quantitative results obtained using the meconium calibration curve showed excellent correlation $(<15 \% \mathrm{CV})$ with those obtained using the calibration curve made up in negative blood and as a result, all subsequent method validation experiments were performed using calibration curves prepared in certified negative blood.

\subsubsection{Working Standards}

Calibration curves were constructed using eight calibration standards prepared in certified drug free whole blood and all sample volumes were $1 \mathrm{~mL}$. Two working standards were used to construct the calibration standards, working standard $1(500 \mathrm{ng} / \mathrm{mL})$ was prepared by combining $150 \mu \mathrm{L}$ of an amphetamine and methamphetamine stock solution $(10 \mu \mathrm{g} / \mathrm{mL})$ with $2850 \mu \mathrm{L}$ of negative blood. Working standard $2(50 \mathrm{ng} / \mathrm{mL})$ was prepared by combining $300 \mu \mathrm{L}$ of working standard 1 with $2700 \mu \mathrm{L}$ of negative blood. An internal standard solution $(500 \mathrm{ng} / \mathrm{mL})$ was prepared using $1 \mathrm{mg} / \mathrm{mL}$ standards of amphetamine- $\mathrm{d}_{6}$ and methamphetamine- $\mathrm{d}_{9}$.

\subsubsection{Calibration Curves}

Calibration curves for all experiments were prepared according to Table 44 . 
Table 44: Preparation of amphetamine and methamphetamine calibration curves.

\begin{tabular}{|c|c|c|}
\hline Standard Concentration $(\mathbf{n g} / \mathbf{m L})$ & Volume of Working Standard $(\boldsymbol{\mu L})$ & Volume of Negative Blood $(\boldsymbol{\mu L})$ \\
\hline 500 & $1000(\operatorname{Std} 1)$ & 0 \\
\hline 250 & $500(\operatorname{Std} 1)$ & 500 \\
\hline 100 & $200(\operatorname{Std} 1)$ & 800 \\
\hline 50 & $1000(\operatorname{Std} 2)$ & 0 \\
\hline 25 & $500(\operatorname{Std} 2)$ & 500 \\
\hline 10 & $200(\operatorname{Std} 2)$ & 800 \\
\hline 5 & $100(\operatorname{Std} 2)$ & 900 \\
\hline Negative & 0 & 1000 \\
\hline
\end{tabular}

$100 \mu \mathrm{L}$ of internal standard solution $(500 \mathrm{ng} / \mathrm{mL})$ was added to each sample including the blank and all samples were then vortexed for 30 seconds.

\subsubsection{Quality Control (QC) Standards}

Quality control standards (QC's) were prepared and analyzed with every batch to ensure the accurate identification and quantitation of validation specimens. A low QC (LQC) was prepared at a concentration of $5.0 \mathrm{ng} / \mathrm{mL}$ and a high QC (HQC) at a concentration of $500 \mathrm{ng} / \mathrm{mL}$. Quality control standards were injected immediately following the final point of the calibration curve and quantitation of each QC was required to be accurate within $20 \%$ of the expected nominal value to proceed with the analysis of validation specimens.

\subsubsection{Sample Preparation}

Meconium samples were accurately weighed and then diluted by a factor of $3(\mathrm{w} / \mathrm{v})$ with 50:50 methanol/water to assist with the sonication procedure. Samples were shaken and sonicated for 10-15 minutes. Following sonication, $1 \mathrm{~mL}$ of the meconium samples was added to appropriately labeled culture tubes. Amphetamine and methamphetamine standards and deuterated internal standards were added to samples which were then block vortexed for 5 minutes. Samples were centrifuged for 5 minutes at $3500 \mathrm{rpm}$ after which the supernatants were transferred to appropriately labeled culture tubes $(12 \times 75)$. Sample recovery was optimized by 
adding $500 \mu \mathrm{L}$ of $2.0 \mathrm{M} \mathrm{NaOH}$ to ensure that the analyte was present in its basic state. Samples were then diluted with $2 \mathrm{~mL}$ of deionized water and vortexed for 10-15 seconds. Samples were loaded into 5mL ISOLUTE HM-N supported liquid extraction columns and left to sit for 10 minutes. Analytes were initially eluted with $5 \mathrm{~mL}$ of ethyl acetate and after a 3 minute waiting period a second elution step was performed with $3 \mathrm{~mL}$ of ethyl acetate. $200 \mu \mathrm{L}$ of $1 \% \mathrm{HCl}$ was added to all eluates to ensure formation of the hydrochloride salts to reduce the possibility of analyte loss during evaporation steps. Samples were dried down under a gentle stream of nitrogen and reconstituted in $200 \mu \mathrm{L}$ of DI water. Samples were transferred to UPLC vials and injected.

\subsubsection{Liquid Chromatography}

Liquid chromatographic separations were performed on a Waters ACQUITY ${ }^{\mathrm{TM}}$ ultra performance liquid chromatograph (UPLC ${ }^{\mathrm{TM}}$ ) (Waters Corp., Milford, MA, USA). Separations were achieved on an ACQUITY UPLC ${ }^{\text {TM }}$ phenyl column (2.1x 50mm) packed with $1.7 \mu \mathrm{m}$ bridged ethyl hybrid (BEH) particles and maintained at $35^{\circ} \mathrm{C}$. The mobile phase consisted of deionized water containing $0.1 \%$ formic acid (solvent A), and acetonitrile containing $0.1 \%$ formic acid (solvent B). Analyte elution was achieved using the following step-wise binary elution gradient: Initial mobile phase composition was 99:1 $\left(\mathrm{H}_{2} \mathrm{O}: \mathrm{ACN}\right)$. The composition of solvent B was increased to $2 \%$ over the first 0.10 mins after which time it was linearly increased to $10 \%$ over 2.9 mins followed by an increase to $100 \%$ over 0.50 mins. Conditions were returned to their initial composition of 99:1 (H2O:ACN) over 0.50 mins and held for 1 min to equilibrate the column before the next injection. The total run time was 5 mins. Samples were maintained at $10^{\circ} \mathrm{C}$ in the sample organizer and sample injection volumes were $1 \mu \mathrm{L}$ for all analyses. Flow rates 
remained constant at $0.5 \mathrm{~mL} / \mathrm{min}$ and all flow was directed into the ESI source of the mass spectrometer.

\subsubsection{Mass Spectrometry}

Mass spectrometry conditions were as follows: capillary voltage $0.60 \mathrm{kV}$, cone voltage $22 \mathrm{~V}$, extractor voltage $3.1 \mathrm{~V}$, RF lens voltage $0.1 \mathrm{~V}$. The source temperature was $150^{\circ} \mathrm{C}$ while the desolvation temperature was set at $350^{\circ} \mathrm{C}$. Cone gas was set at a flow of $50 \mathrm{~L} / \mathrm{Hr}$ while the desolvation gas flow was $900 \mathrm{~L} / \mathrm{Hr}$. The collision gas flow was set to $0.18 \mathrm{~mL} / \mathrm{min}$. Nitrogen (99.995\% purity) was used as the desolvation gas, and ultra-pure argon (99.999\% purity) was used as the collision gas. Table 45 reports the mass transitions, dwell times, cone voltages, and collision energies for each of the analytes and their deuterated internal standards.

Table 45: MS/MS parameters used for each analyte and deuterated internal standard.

\begin{tabular}{|c|c|c|c|c|c|}
\hline Compound & Mass transition & Purpose & Cone (V) & Collision (V) & Dwell (secs) \\
\hline Amphetamine & $135.97>90.90$ & Quantifying ion & 20 & 14 & 0.02 \\
\hline Amphetamine & $135.97>119.0$ & Qualifying ion & 20 & 10 & 0.02 \\
\hline Amphetamine-d6 & $141.94>93.00$ & Quantifying ion & 20 & 16 & 0.02 \\
\hline Methamphetamine & $149.97>90.90$ & Quantifying ion & 25 & 16 & 0.02 \\
\hline Methamphetamine & $149.97>119.0$ & Qualifying ion & 25 & 12 & 0.02 \\
\hline Methamphetamine-d9 & $159.03>92.90$ & Quantifying ion & 25 & 18 & 0.02 \\
\hline
\end{tabular}

Figure 30 illustrates the fragmentation pathways for amphetamine and methamphetamine under ESI conditions and the resulting product ions for analyte detection in MRM experiments. 
Amphetamine

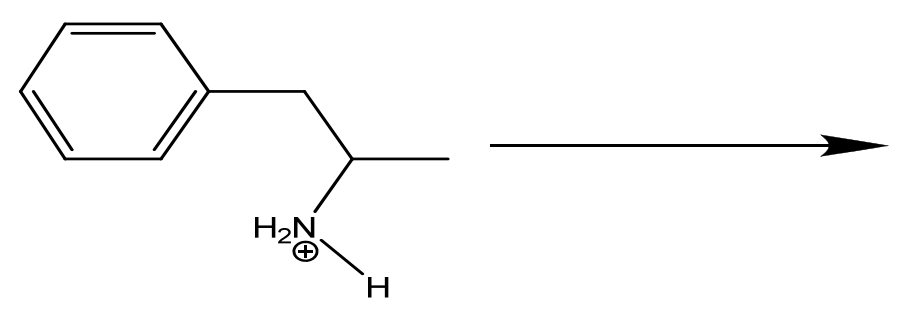

Chemical Formula: $\mathrm{C}_{9} \mathrm{H}_{14} \mathrm{~N}^{+}$

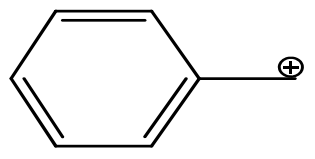

Chemical Formula: $\mathrm{C}_{7} \mathrm{H}_{7}^{+}$

Molecular Weight: 91.13

Molecular Weight: 136.21

Methamphetamine
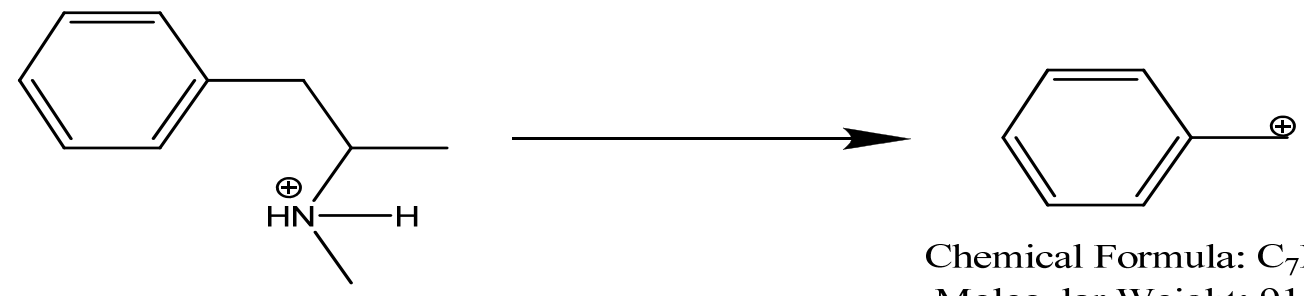

Chemical Formula: $\mathrm{C}_{7} \mathrm{H}_{7}^{+}$

Chemical Formula: $\mathrm{C}_{10} \mathrm{H}_{16} \mathrm{~N}^{+}$

Molecular Weight: 91.13

Molecular Weight: 150.24

Figure 30: Fragmentation pathways for amphetamine and methamphetamine during tandem mass spectrometry experiments[261].

\subsection{Results and Discussion}

During direct infusion experiments, the most abundant product ion for both amphetamine and methamphetamine was identified as the $\mathrm{m} / \mathrm{z} 90.90$ ion (Figure 30 ). Subsequently, the mass transition from 135.97>90.90 was monitored as the quantifying trace for amphetamine and the mass transition from $149.97>90.90$ was monitored as the quantifying trace for methamphetamine. In order to monitor these mass transitions, MS1 was set to pass masses of 135.97 and 149.97 along with the parent masses of the deuterated internal standards. Following collision induced dissociation of the protonated molecular ions into their characteristic product ions, MS2 was set to transmit only product ions with $\mathrm{m} / \mathrm{z} 90.90$. To eliminate the potential effects of cross-talk between the identical fragment ions of amphetamine and methamphetamine, attempts were made at developing a chromatographic run capable of baseline resolving the two 
analytes. Due to their structural similarities, amphetamine and methamphetamine exhibit almost identical physicochemical properties. Initial UPLC experiments indicated that the baseline resolution of the two compounds could only be achieved with a non-traditional step-wise gradient.

\subsubsection{Chromatographic Separation of Amphetamine and Methamphetamine}

Preliminary chromatographic experiments aimed to chromatographically resolve amphetamine and methamphetamine to eliminate the possibility of ion 'cross talk' which can complicate the quantitation process. The initial elution gradient profile was as follows: $0-0.10$ $\min , 25 \% \mathrm{~B} ; 0.10-3.0 \mathrm{~min}, 25-100 \% \mathrm{~B} ; 3.0-3.5 \mathrm{~min}$, return to initial conditions; $3.5-5.0$ min, equilibration of the column. The chromatographic separation under these conditions exhibited poor resolution (Figure 31). 


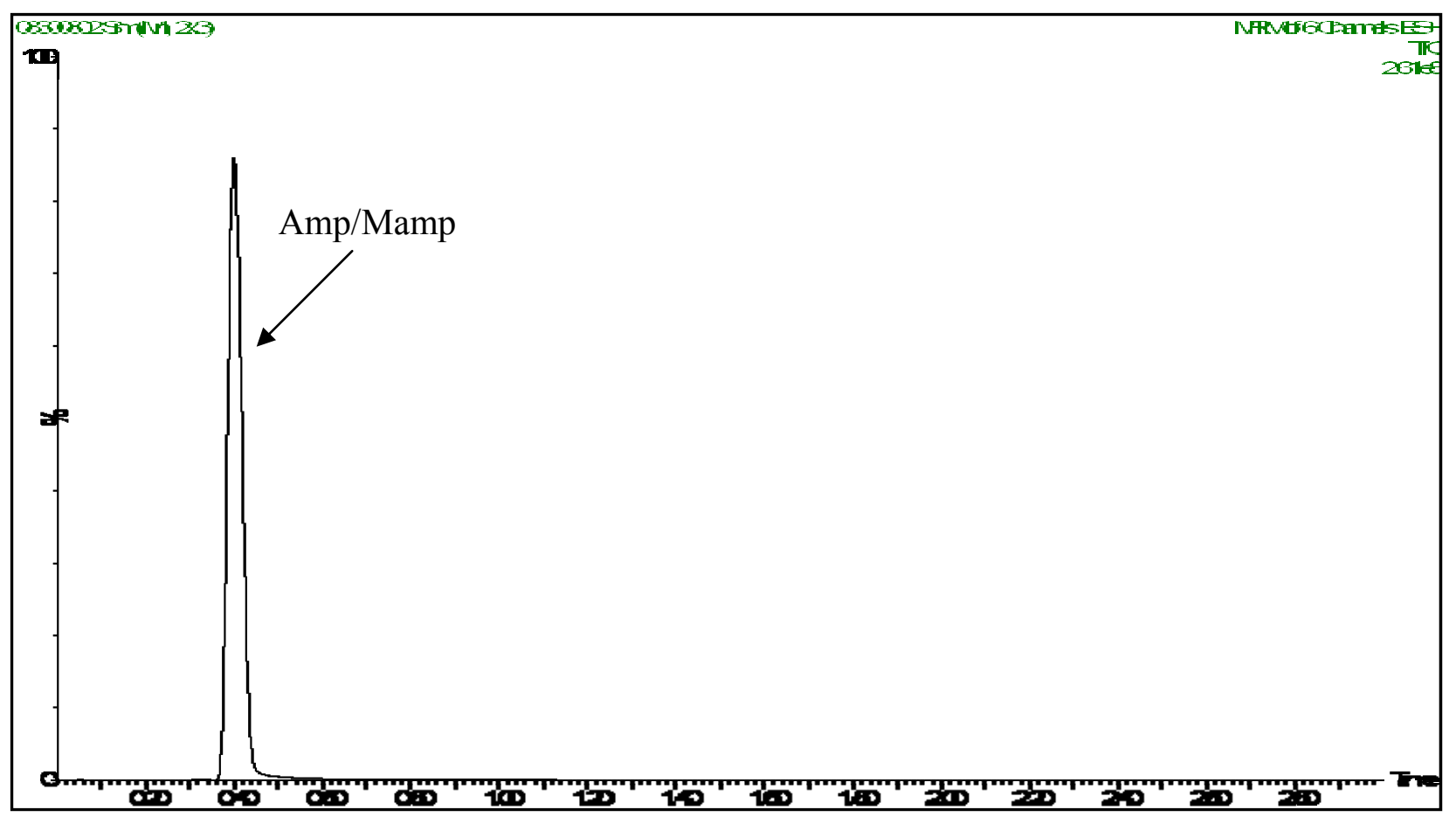

Figure 31: TIC of UPLC separation \#1. Elution gradient failed to resolve amphetamine (Amp) and methamphetamine (Mamp).

In order to achieve baseline resolution of amphetamine from methamphetamine, a second gradient profile was evaluated. The elution gradient profile was as follows: $0-0.10 \mathrm{~min}, 15 \% \mathrm{~B}$; $0.10-3.0 \mathrm{~min}, 15-100 \% \mathrm{~B} ; 3.0-3.5 \mathrm{~min}$, return to initial conditions; $3.5-5.0 \mathrm{~min}$, equilibration of the column. The chromatographic separation of amphetamine and methamphetamine improved under these conditions however significant co-elution meant that quantitation could still prove inaccurate as a result of ion 'cross talk' (Figure 32). 


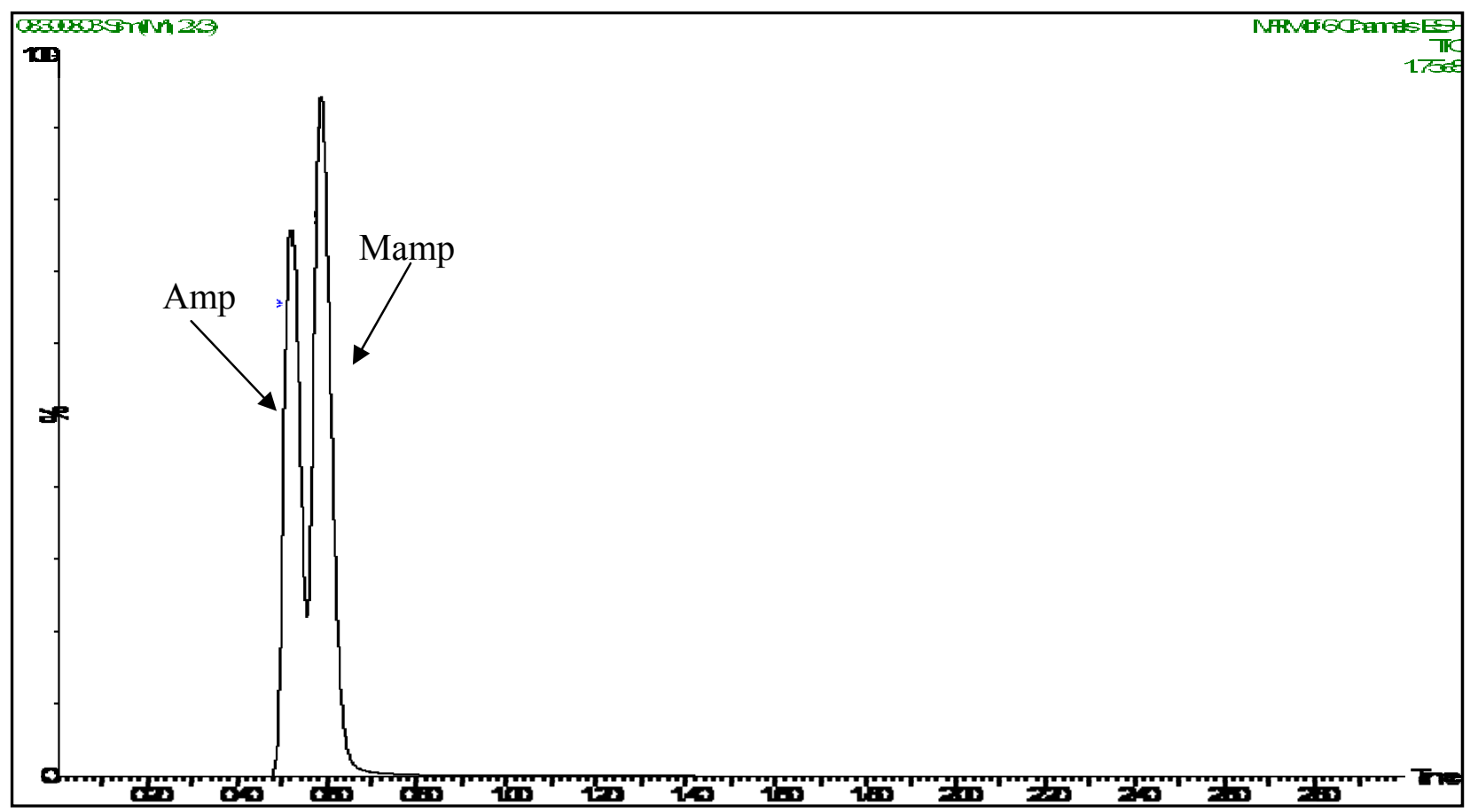

Figure 32: TIC of UPLC separation \#2. Elution gradient resulted in the co-elution of amphetamine (Amp) and methamphetamine (Mamp).

Chromatographic resolution was improved using the second elution gradient profile in which the initial aqueous component was increased. In order to investigate the effect of increasing the aqueous component further, a third gradient profile was evaluated. The elution gradient profile was as follows: $0-0.10 \mathrm{~min}, 5-10 \% \mathrm{~B} ; 0.10-3.0 \mathrm{~min}, 10-100 \% \mathrm{~B} ; 3.0-3.5 \mathrm{~min}$, return to initial conditions; $3.5-5.0 \mathrm{~min}$, equilibration of the column. Chromatographic resolution was not significantly improved using an increased aqueous component (Figure 33). 


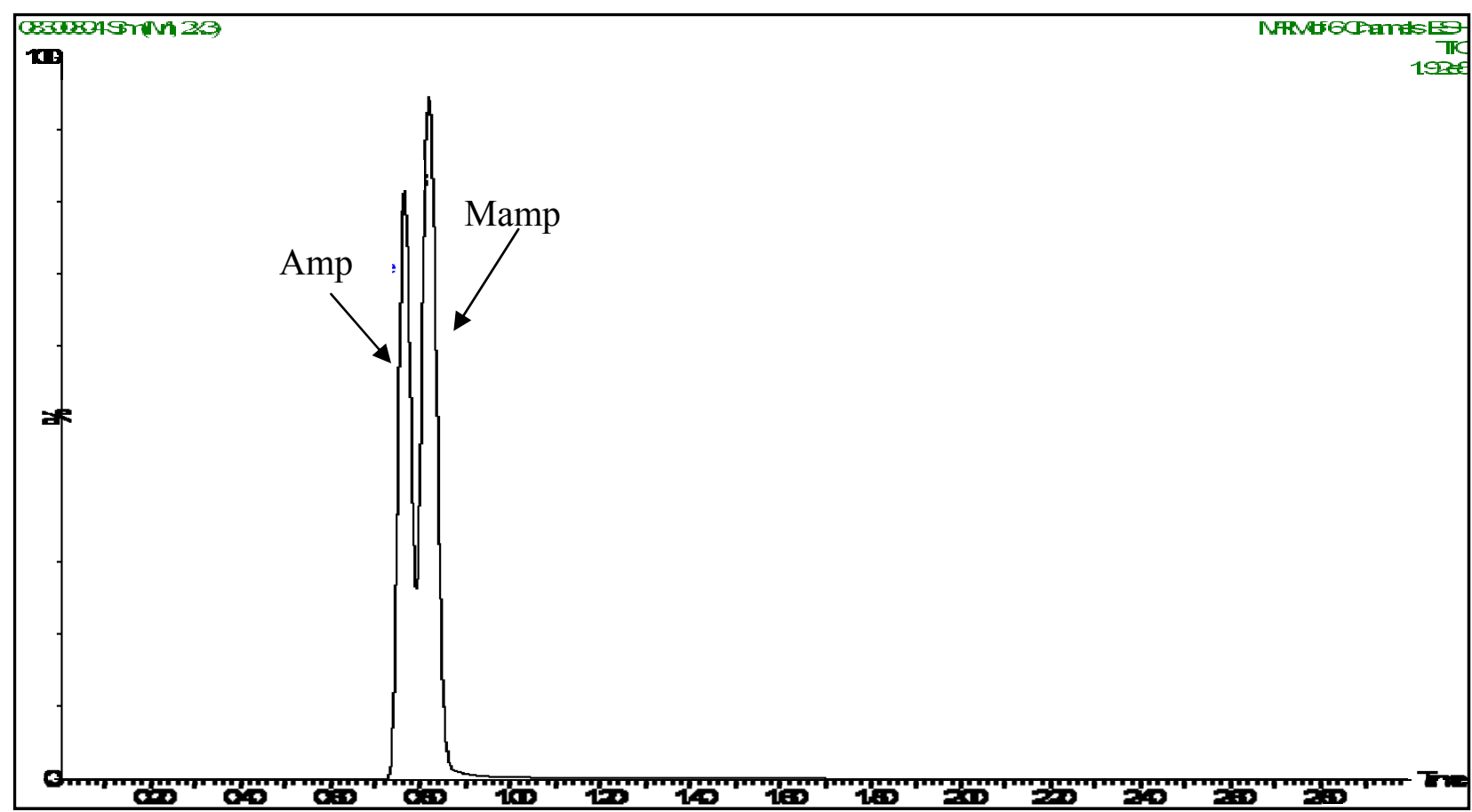

Figure 33: TIC of UPLC separation \#3. Elution gradient was unable to resolve amphetamine (Amp) and methamphetamine (Mamp).

As an increase in the initial aqueous component of the gradient profile did not significantly improve the chromatographic resolution, the effect of sustaining a slightly higher aqueous component in the early stage of the separation was investigated. The elution gradient profile was as follows: $0-0.10 \mathrm{~min}, 1 \% \mathrm{~B} ; 0.10-3.0 \mathrm{~min}, 1-100 \% \mathrm{~B} ; 3.0-3.5 \mathrm{~min}$, return to initial conditions; $3.5-5.0 \mathrm{~min}$, equilibration of the column. The higher aqueous composition again shifted the retention times of both analytes but did not significantly improve the chromatographic separation (Figure 34). 


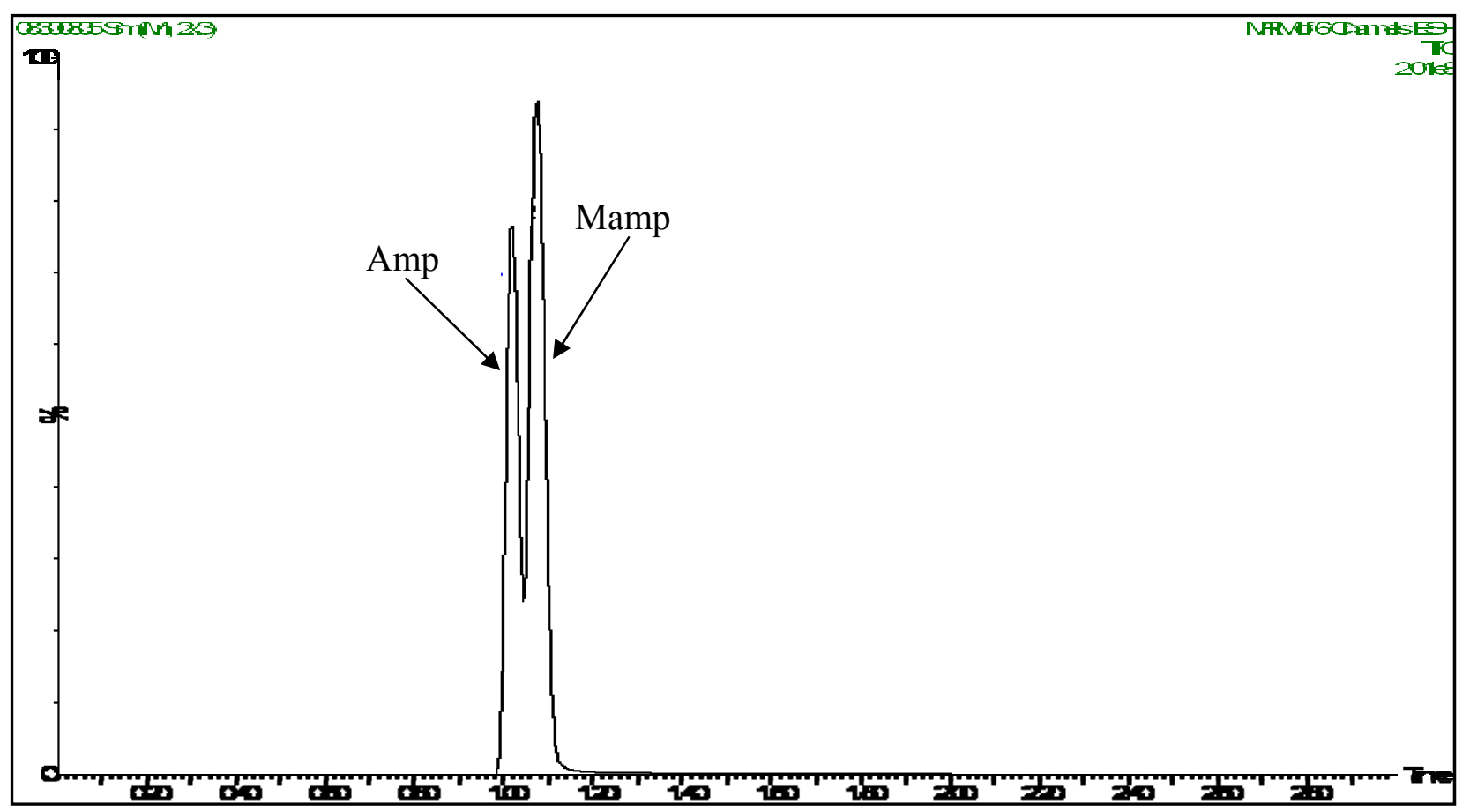

Figure 34: TIC of UPLC separation \#4. Elution gradient was unable to resolve amphetamine (Amp) and methamphetamine (Mamp).

In order to evaluate the separation power of a largely aqueous mobile phase, the elution gradient profile was modified to sustain a largely aqueous composition not only during the early stages of the separation but also throughout and beyond analyte elution. The elution gradient profile was as follows: $0-0.10 \mathrm{~min}, 1-5 \% \mathrm{~B} ; 0.10-3.0 \mathrm{~min}, 5-10 \% \mathrm{~B} ; 3.0-3.5 \mathrm{~min}, 10-90 \% \mathrm{~B} ; 3.50$ - 4.50 min, return to initial conditions; $4.5-5.5 \mathrm{~min}$, equilibration of the column. While chromatographic resolution of amphetamine and methamphetamine was achieved using the highly aqueous mobile phase, significant peak broadening, especially in the case of methamphetamine, meant that quantitation was unreliable and often inaccurate (Figure 35). 


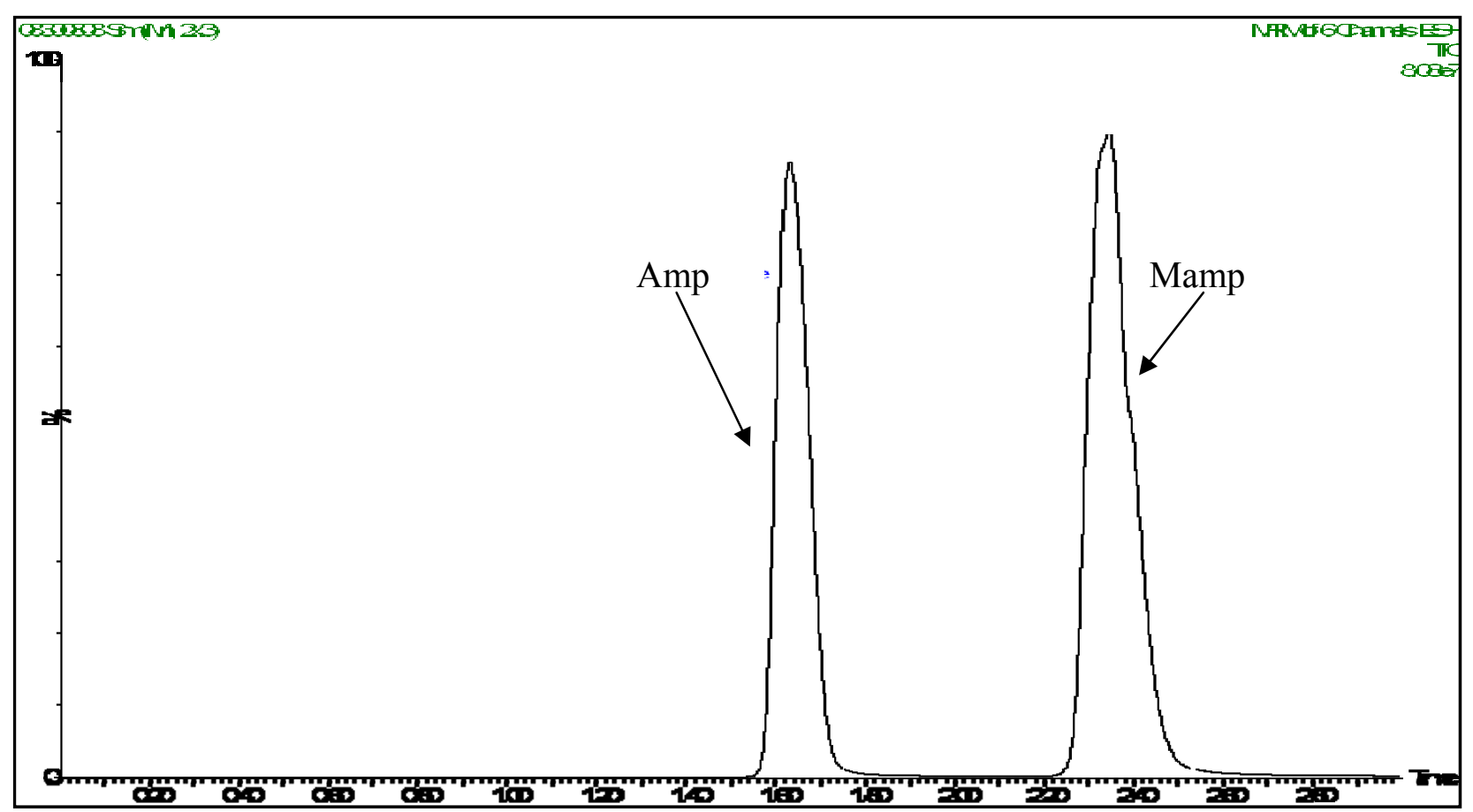

Figure 35: TIC of UPLC separation \#5. Elution gradient resulted in significant peak broadening.

In order to minimize peak broadening while still achieving baseline resolution, the following elution gradient profile was evaluated: $0-0.10 \mathrm{~min}, 1-2 \% \mathrm{~B} ; 0.10-3.0 \mathrm{~min}, 2-10 \% \mathrm{~B} ; 3.0-$ $3.5 \mathrm{~min}, 10-100 \% \mathrm{~B} ; 3.50-4.0 \mathrm{~min}$, return to initial conditions; $4.0-5.0 \mathrm{~min}$, equilibration of the column. The elution gradient maintained a higher aqueous component during the initial stage of the separation and increased the organic component during the ramp between 3.0 and 3.5 minutes. Band broadening was significantly reduced without significantly altering retention times (Figure 36). Both analytes eluted in less than three minutes and the total run time was five minutes (Figure 36). This elution gradient profile was employed for the remainder of validation experiments. 


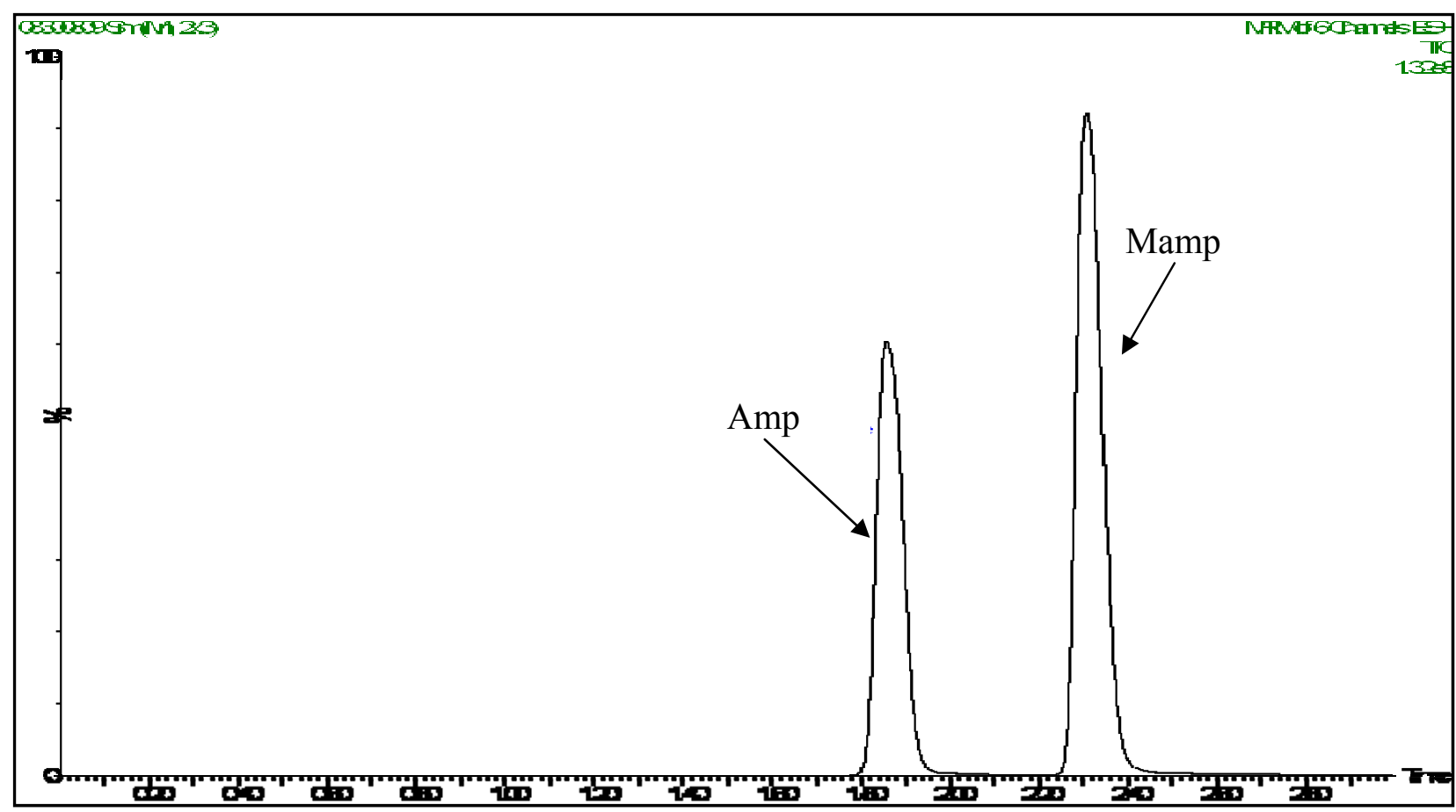

Figure 36: TIC of UPLC separation \#6. Elution gradient allowed for the baseline resolution of amphetamine (Amp) and methamphetamine (Mamp).

\subsubsection{Method Validation}

Equivalence studies were performed to investigate the accuracy of employing calibration curves constructed in certified drug-free blood for the purpose of quantifying analytes in the meconium matrix. Five LQC and five HQC standards were prepared and quantified using a calibration curve constructed in negative whole blood, and a calibration curve constructed in meconium which had previously screened negative for the analytes of interest. The relative error of the two quantitative values was calculated for each QC and used to determine the level of agreement between the two calibration curves. Relative error did not exceed $2 \%$ indicating a high degree of correlation between calibration curves constructed in blood and calibration curves constructed in meconium for the purpose of quantifying amphetamine and methamphetamine in the meconium matrix (Table 46 and 47). Calibration curves constructed in negative blood appear 
to generate accurate quantitations when applied to the meconium matrix which is encouraging.

Calibration curves constructed in certified drug-free blood were deemed suitable for the quantitation of amphetamine and methamphetamine in the meconium matrix and were subsequently employed for the remainder of validation experiments.

Table 46: Amphetamine equivalence studies using quantitative values obtained from calibration curves constructed in meconium (MC) and blood (BC).

\begin{tabular}{|c|c|c|c|}
\hline Amphetamine Std & $* \mathbf{B C}(\mathbf{n g} / \mathbf{m L})$ & $* \mathbf{M C}(\mathbf{n g} / \mathbf{m L})$ & $\mathbf{\% R E}$ \\
\hline LQC & 10.0 & 10.1 & $1.0 \%$ \\
\hline HQC & 513.4 & 506.7 & $1.3 \%$ \\
\hline NEG(B) & 0 & 0 & N/A \\
\hline NEG(M) & 0 & 0 & N/A \\
\hline
\end{tabular}

*Reported concentrations are mean values of 5 replicate analyses.

Table 47: Methamphetamine equivalence studies using quantitative values obtained from calibration curves constructed in meconium (MC) and blood (BC).

\begin{tabular}{|c|c|c|c|}
\hline Methampheatmine Std & ${ }^{*} \mathbf{B C}(\mathbf{n g} / \mathbf{m L})$ & $\left.{ }^{*} \mathbf{M C} \mathbf{( n g} / \mathbf{m L}\right)$ & $\mathbf{\% R E}$ \\
\hline LQC & 9.8 & 9.4 & $4.3 \%$ \\
\hline HQC & 538.7 & 503.3 & $7.0 \%$ \\
\hline NEG(B) & 0 & 0 & N/A \\
\hline NEG(M) & 0 & 0 & N/A \\
\hline
\end{tabular}

*Reported concentrations are mean values of 5 replicate analyses.

The analytical methodology was deemed selective following the analysis of five meconium blanks and five QC standards prepared at the LLOQ which had been spiked with various exogenous interferences commonly encountered in forensic specimens (Table 48). Blank meconium specimens were analyzed to ensure minimal analyte response was generated from any endogenous matrix components. Analysis of five LLOQ specimens which had been spiked with potential interfering species was performed to ensure selectivity at the low end of the calibration range and to eliminate the possibility of false positives generated from exogenous interferences. 
Table 48: Exogenous compounds included in selectivity quality control standards.

\begin{tabular}{|c|l|}
\hline Standard & \multicolumn{1}{|c|}{ Contents } \\
\hline Selectivity QC 1 & $\begin{array}{l}\text { Carbamazepine, carbamazepine epoxide, felbamate, gabapentin, lamotrigine, } \\
\text { levetiracetam, mephobarbital, naproxen, oxcarbazepine, phenytoin, primidone, topiramate, } \\
\text { valproic acid, zonisamide }\end{array}$ \\
\hline Selectivity QC 2 & $\begin{array}{l}\text { Amitriptyline, bupropion, clomipramine, desipramine, doxepin, fluoxetine, imipramine, } \\
\text { norclomipramine, nordoxepin, norfluoxetine, norsertraline, nortriptyline, norvenlafaxine, } \\
\text { paroxetine, sertraline, venlafaxine }\end{array}$ \\
\hline Selectivity QC 3 & $\begin{array}{l}\text { Alprazolam, chlorpheniramine, citalopram, clonazepam, cyclobenzaprine, } \\
\text { dextromethorphan, duloxetine, fentanyl, flunitrazepam, haloperidol, mirtazapine, } \\
\text { olanzapine, strychnine, zolpidem }\end{array}$ \\
\hline
\end{tabular}

Analysis of blank meconium specimens indicated that endogenous matrix components are capable of generating only minimal detector responses which correspond to concentrations well below the LLOQ. Analyte responses during the analysis of blank samples did not exceed $0.2 \mathrm{ng} / \mathrm{mL}$ for either analyte (Tables 49 and 50). Results indicate that false positives arising from endogenous matrix interferences are unlikely. Analysis of QC standards prepared at the LLOQ, which had been spiked with various exogenous interferences, indicated that accurate and selective identification of amphetamine and methamphetamine was possible even in the presence of various other xenobiotics. Quantitation accuracies of spike QC standards ranged from $96 \%$ to $100 \%$ for amphetamine and $94 \%$ to $98 \%$ for methamphetamine (Table $49 \& 50$ ). Accurate quantitations at the LLOQ for both analytes were possible due to the high selectivity of the tandem mass spectrometric methodology. Selectivity studies indicate that false positives due to matrix interferences or potentially interfering exogenous species are not likely. 
Table 49: Amphetamine selectivity studies using blank meconium from five different sources and blank meconium specimens spiked with various exogenous interferences.

\begin{tabular}{|c|c|c|c|c|}
\hline Amphetamine Std & $\begin{array}{c}\text { Std Conc } \\
\text { (ng/mL) }\end{array}$ & $*$ Measured Conc (ng/mL) & Acceptable range & Pass/Fail \\
\hline Blank samples & 0 & 0.1 & $<5 \mathrm{ng} / \mathrm{mL}$ & Pass \\
\hline Spiked samples & 5 & 5.0 & $5+/-20 \%$ & Pass \\
\hline
\end{tabular}

*Reported concentrations are mean values of 5 replicate analyses.

Table 50: Methamphetamine selectivity studies using blank meconium from five different sources and blank meconium specimens spiked with various exogenous interferences.

\begin{tabular}{|c|c|c|c|c|}
\hline Methamphetamine Std & Std Conc & ${ }^{*}$ Measured Conc (ng/mL) & Acceptable range & Pass/Fail \\
\hline Blank samples & 0 & 0.2 & $<5 \mathrm{ng} / \mathrm{mL}$ & Pass \\
\hline Spiked samples & 5 & 5.2 & $5+/-20 \%$ & Pass \\
\hline
\end{tabular}

*Reported concentrations are mean values of 5 replicate analyses.

The accuracy of the analytical method was investigated by analyzing five replicate QC standards over three different concentrations spanning the calibration range. Accuracy was assessed by calculating the closeness of the mean test results to the known standard concentration. Mean values were determined using five replicates prepared at concentrations of 500, 50, and $5 \mathrm{ng} / \mathrm{mL}$. Mean values of $491.1 \mathrm{ng} / \mathrm{mL}, 50.8 \mathrm{ng} / \mathrm{mL}$, and $4.8 \mathrm{ng} / \mathrm{mL}$ were obtained from replicate analysis of amphetamine standards at concentrations of 500, 50, and $5 \mathrm{ng} / \mathrm{mL}$ respectively, representing accuracies of $98.2 \%, 98.4 \%$, and $96 \%$ (Table 51 ). Replicate analysis of methamphetamine QCs prepared at concentrations of 500, 50, and $5 \mathrm{ng} / \mathrm{mL}$ produced mean test values of $530.9 \mathrm{ng} / \mathrm{mL}, 53.2 \mathrm{ng} / \mathrm{mL}$, and $4.7 \mathrm{ng} / \mathrm{mL}$ respectively, representing accuracies of 93.8\%, 93.6\%, and 94\% (Table 52). 
Table 51: Amphetamine accuracy studies.

\begin{tabular}{|c|c|c|c|}
\hline Amphetamine Std & Std Conc (ng/mL) & ${ }^{*}$ Measured value (ng/mL) & \%RE \\
\hline High point & 500 & 491.1 & $1.8 \%$ \\
\hline Midpoint & 50 & 50.8 & $1.6 \%$ \\
\hline LLOQ & 5 & 4.8 & $4.0 \%$ \\
\hline
\end{tabular}

*Reported concentrations are mean values of 5 replicate analyses.

Table 52: Methamphetamine accuracy studies.

\begin{tabular}{|c|c|c|c|}
\hline Methamphetamine Std & Std Conc $(\mathbf{n g} / \mathbf{m L})$ & ${ }^{*}$ Measured value $(\mathbf{n g} / \mathbf{m L})$ & \%RE \\
\hline High point & 500 & 530.9 & $6.2 \%$ \\
\hline Midpoint & 50 & 53.2 & $6.4 \%$ \\
\hline LLOQ & 5 & 4.7 & $6.0 \%$ \\
\hline
\end{tabular}

*Reported concentrations are mean values of 5 replicate analyses.

Precision of the analytical methodology was investigated by analyzing five replicate QC standards at concentrations of 500,50 , and $5 \mathrm{ng} / \mathrm{mL}$ over four consecutive days. Both intra- and inter-batch studies indicated high method precision $(\mathrm{CVs}<8.5 \%)$. Intra-batch precision studies yielded CVs ranging from $1.4 \%-8.5 \%$ for amphetamine and $0.7 \%-5.4 \%$ for methamphetamine indicating high intra-batch precision over the entire calibration range. (Table $53 \& 54)$. Interbatch precision studies were also promising with four-day CVs of $3.9 \%, 2.0 \%$, and $6.2 \%$ for amphetamine QCs prepared at concentrations of 500, 50, and $5 \mathrm{ng} / \mathrm{mL}$, while analysis of methamphetamine standards over the four day time period produced CVs of $1.9 \%, 3.7 \%$, and $5.3 \%$ for the 500,50 , and $5 \mathrm{ng} / \mathrm{mL}$ QCs respectively (Table $53 \& 54$ ).

Table 53: Intra- and inter-batch precision studies for amphetamine.

\begin{tabular}{|c|c|c|c|c|c|c|}
\hline Amphetamine & *Day 1 value & *Day 2 value & *Day 3 value & *Day 4 value & 4 day mean & 4 day CV \\
\hline Highpoint & 491.1 & 494.7 & 530.1 & 523.8 & 509.9 & $3.9 \%$ \\
\hline CV & $1.6 \%$ & $3.2 \%$ & $2.7 \%$ & $1.4 \%$ & & \\
\hline Midpoint & 50.8 & 49.8 & 51.3 & 48.9 & 50.2 & $2.0 \%$ \\
\hline CV & $1.8 \%$ & $1.9 \%$ & $1.4 \%$ & $5.3 \%$ & & \\
\hline LLOQ & 4.8 & 4.6 & 5.1 & 5.3 & 5.0 & $6.2 \%$ \\
\hline CV & $5.6 \%$ & $8.5 \%$ & $4.5 \%$ & $2.6 \%$ & & \\
\hline
\end{tabular}

*Reported concentrations are mean values of 5 replicate analyses. 
Table 54: Intra- and inter-batch precision studies for methamphetamine.

\begin{tabular}{|c|c|c|c|c|c|c|}
\hline Mamp & *Day 1 value & *Day 2 value & *Day 3 value & *Day 4 value & 4 day mean & 4 day CV \\
\hline Highpoint & 530.9 & 507.7 & 519.9 & 524.9 & 520.9 & $1.9 \%$ \\
\hline CV & $1.6 \%$ & $1.6 \%$ & $0.7 \%$ & $3.2 \%$ & & \\
\hline Midpoint & 53.2 & 49.7 & 52.3 & 49.3 & 51.1 & $3.7 \%$ \\
\hline CV & $0.9 \%$ & $1.6 \%$ & $2.7 \%$ & $4.3 \%$ & & \\
\hline LLOQ & 4.7 & 4.6 & 4.9 & 5.2 & 4.9 & $5.3 \%$ \\
\hline CV & $3.8 \%$ & $5.4 \%$ & $2.7 \%$ & $2.9 \%$ & & \\
\hline
\end{tabular}

*Reported concentrations are mean values of 5 replicate analyses.

Recovery of amphetamine and methamphetamine from the meconium matrix using the HM-N supported-liquid extraction technique was investigated to determine the efficiency of the extraction. Extraction efficiency was assessed by comparing the detector response for unextracted standards prepared at concentrations of 500,50 , and $5 \mathrm{ng} / \mathrm{mL}$ with the detector response for extracted standards prepared at the same concentrations. Standards were prepared in triplicate and peak area responses for unextracted standards represented $100 \%$ recovery. Peak area responses for extracted standards were then used to calculate recovery. Mean analyte recoveries over the three concentrations investigated were $52 \%$ for amphetamine and $52.7 \%$ for methamphetamine (Figure $37 \& 38$ ). Mean analyte recoveries for triplicate standards at each of the three concentrations ranged from $49-57 \%$ for amphetamine and $47-58 \%$ for methamphetamine. Analyte recovery was extremely consistent and precise and recoveries of greater than $50 \%$ ensure acceptable method sensitivity. 


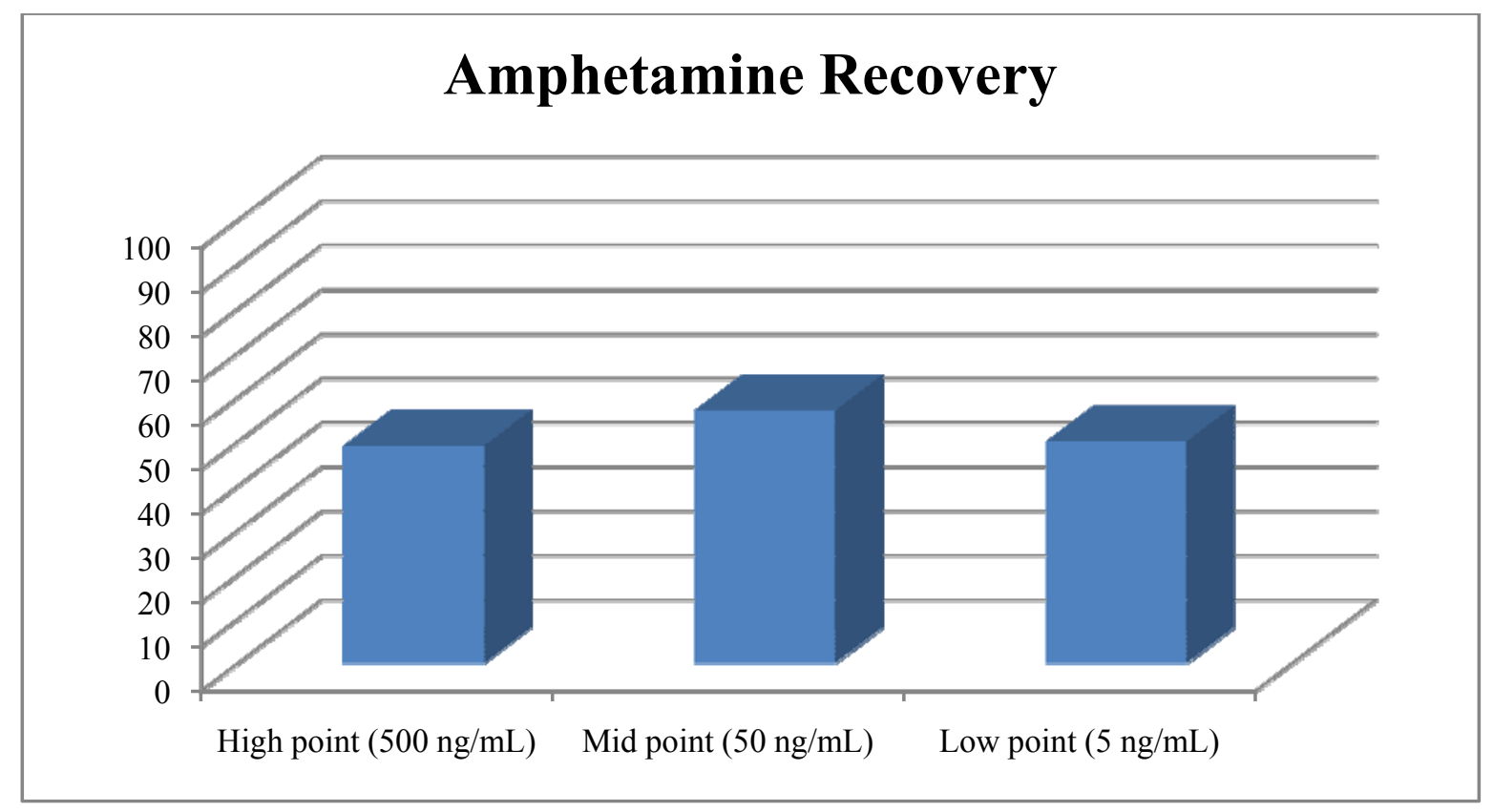

Figure 37: Amphetamine recovery.

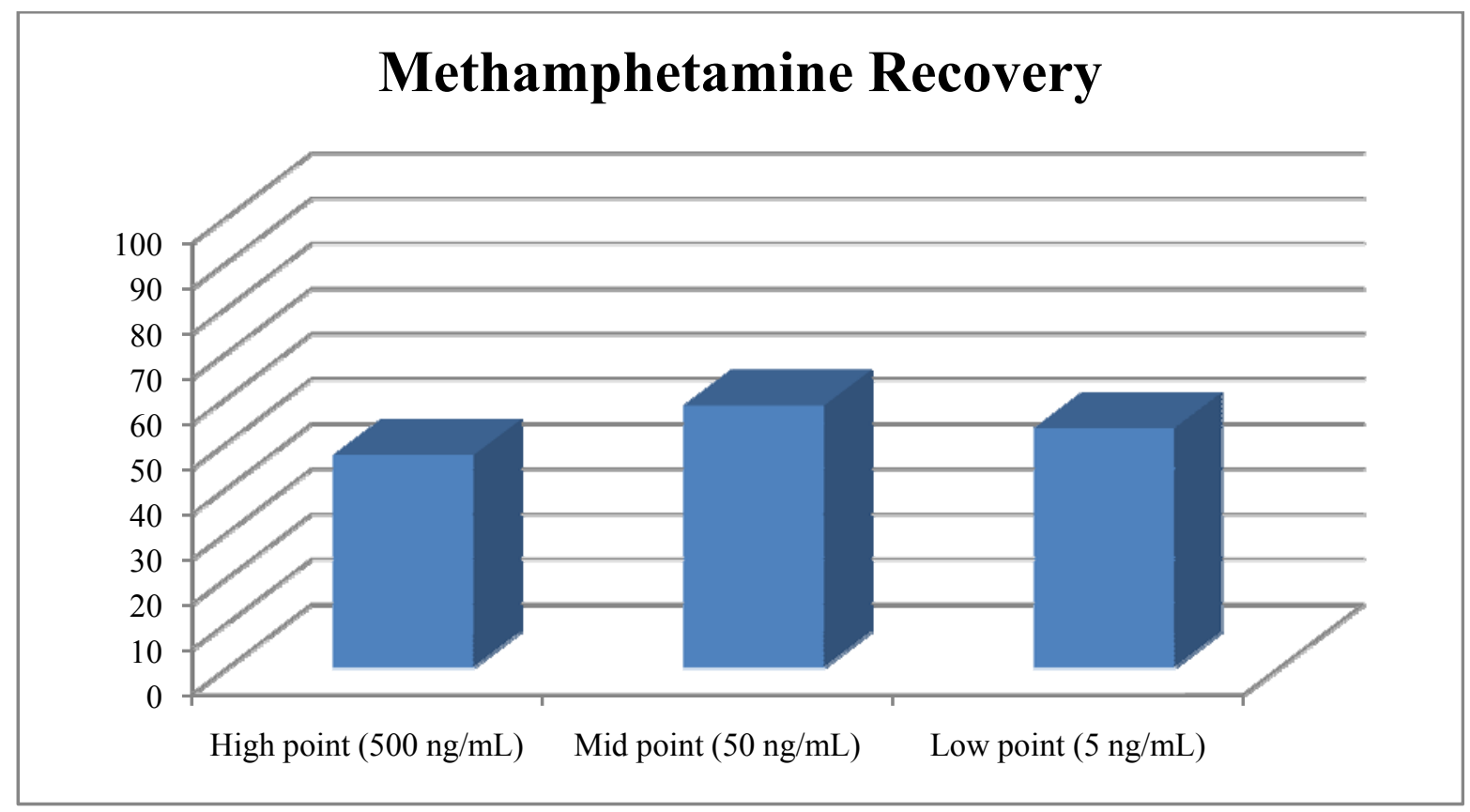

Figure 38: Methamphetamine recovery. 
The limit of detection was $1.0 \mathrm{ng} / \mathrm{mL}$ for amphetamine and $0.250 \mathrm{ng} / \mathrm{mL}$ for methamphetamine corresponding to concentrations of $4.0 \mathrm{ng} / \mathrm{g}$ and $1 \mathrm{ng} / \mathrm{g}$ in the meconium specimen prior to sonication. Limits of detection were calculated based on acceptance criteria for retention times and ion ratios. At concentrations below $1 \mathrm{ng} / \mathrm{mL}$ for amphetamine and $0.250 \mathrm{ng} / \mathrm{mL}$ for methamphetamine, unequivocal identification was not possible due to inaccurate ion ratios. Stability studies indicated that amphetamine and methamphetamine are both stable in the meconium matrix when stored long-term at $4^{\circ} \mathrm{C}$. Stability studies were performed by preparing High and low QC standards which were subsequently subject to a 72 hour freeze-thaw cycle and analyzed following thawing on the third day. Quantitation accuracies during stability studies were greater than $98 \%$ and $99 \%$ for amphetamine LQCs and HQCs respectively, and greater than $98 \%$, and $99 \%$ for methamphetamine low and high QCs respectively. High accuracy and precision during stability studies indicated that current storage temperature and conditions are appropriate for the accurate analysis of authentic meconium specimens.

\subsection{Conclusions}

Meconium is a complex biological matrix that can indicate prenatal exposure to drugs of abuse. Unfortunately, meconium specimens require extensive sample pre-treatment before they are suitable for instrumental analysis. Extensive sample preparation can prove detrimental to sample turnaround time in high throughput toxicology laboratories. ISOLUTE HM-N supported liquid-liquid extraction columns provide an attractive sample pre-treatment technique for the extraction of amphetamine and methamphetamine from meconium specimens. ISOLUTE HM-N columns are designed to deal with difficult matrices such as sonicated meconium specimens which can sometimes cause traditional SPE columns to plug due to sample turbidity. Rapid and selective extraction of both analytes was possible with the columns and subsequent separation 
and quantitation was achieved in less than 5.0 min using UPLC-MS/MS. Two MRM transitions were monitored for each analyte and quantifications were made using deuterated internal standards and seven point calibration curves. Limits of detection were $1 \mathrm{ng} / \mathrm{mL}$ and $250 \mathrm{pg} / \mathrm{mL}$ for amphetamine and methamphetamine, respectively. Injection volumes of $1 \mu \mathrm{L}$ allowed for the linear quantitation of analytes over the range $5 \mathrm{ng} / \mathrm{mL}-500 \mathrm{ng} / \mathrm{mL}$. To the author's knowledge, this is the first validated UPLC-ESI-MS/MS methodology for the quantitation of amphetamine and methamphetamine in meconium specimens. The proposed methodology could greatly reduce the sample preparation time for meconium testing which is becoming an extremely important tool for the identification of prenatal exposure to drugs of abuse. The validated methodology was implemented at AIT Laboratories (Indianapolis, IN) for the purpose of confirming the presence of amphetamine and methamphetamine in authentic meconium specimens which had previously screened positive. From 07/01/08 - 03/06/2009 a total of 4036 meconium specimens were screened for amphetamine/methamphetamine. Of those samples, 138 screened positive representing $3.4 \%$ of the total specimens. 37 of the 138 specimens were confirmed positive using the UPLC-MS/MS method. 


\section{Chapter 8: Investigations into the Application of Desorption Electrospray Ionization (DESI) for the Rapid Screening of Meconium Specimens}

\subsection{Abstract}

The current study investigates the suitability of desorption electrospray ionization (DESI) for the purpose of screening human meconium samples for several cocaine, amphetamine, and opiate derivatives. DESI offers the advantage of ambient sampling meaning that specimens can be directly analyzed following limited sample preparation. Due to the complex nature of the human meconium matrix, significant sample preparation is often required before specimens can be screened using traditional immunoassay techniques. Traditional immunoassay techniques such as ELISA, EMIT, and RIA are designed for qualitatively detecting analytes in common biological matrices such as serum and urine. Direct application of such techniques to the analysis of more complex matrices, such as meconium, can result in inaccurate results and a high rate of false-positives. The suitability of the DESI-MS/MS method for screening meconium specimens was investigated by analyzing 21 authentic specimens that had previously screened positive for either cocaine or amphetamines using an immunoassay screen. Direct comparison of the immunoassay screening results with those obtained using the DESI-MS/MS method indicates that the mass spectral method greatly enhances selectivity and decreases the rate of false positives. Investigations into the suitability of DESI-MS/MS for screening meconium specimens aimed to reduce both the degree of sample preparation and also the number of false-positive results commonly observed with immunoassay techniques. 


\subsection{Introduction}

\subsubsection{Desorption Electrospray Ionization (DESI)}

Desorption electrospray ionization is achieved by directing a fine spray of electrically charged droplets at an ambient object of interest. When the fine spray of droplets hits the surface of interest, desolvated ions created from small organic molecules as well as large biomolecules are released. Analytes initially leave the surface of interest entrained within small, progeny droplets which subsequently evaporate to produce analyte ions. Desorbed ions are subsequently vacuumed through air into a conventional mass spectrometer (Figure 39)[262].

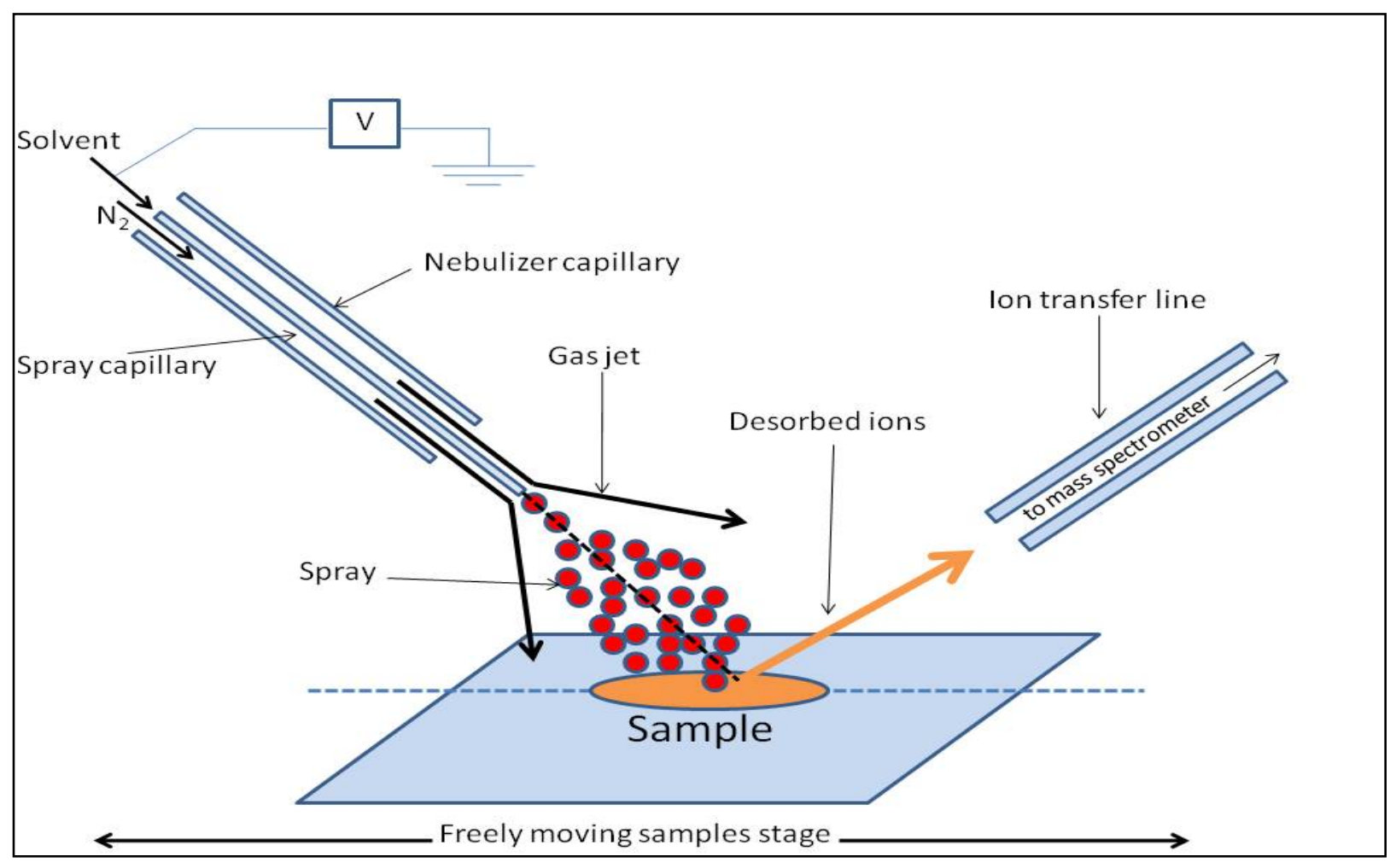

Figure 39: Schematic representation of desorption electrospray ionization (Adapted from Takats 2004). 
DESI was first described in 2004 by Cooks et al who employed the technique for the ionization of peptides and proteins from metal, polymer, and mineral surfaces[263]. The distinguishing feature of DESI is that is allows for the collection of mass spectral data under ambient conditions. Approximately two decades prior to the introduction of DESI by Cooks and co-workers, two new ionization methods were introduced almost simultaneously and have since had an enormous impact on the utilization of mass spectrometry in biology and the life sciences[264]. One of these methods, employed primarily for the analysis of solids, is a desorption ionization (DI) technique known as matrix assisted laser desorption ionization (MALDI)[265]. The second technique, applicable to the analysis of solutions is electrospray ionization (ESI)[266]. Although ionization techniques based upon matrix-assisted laser desorption and electrospray ionization have origins dating back some thirty years, the recognition of their potential for the analysis of large biomolecules has only been realized recently[267]. Both MALDI and ESI are soft ionization techniques meaning that ions produced using either method will possess low internal energies and thus undergo little fragmentation[138]. Both methods exhibit very high ionization efficiencies and allow for very precise $\mathrm{M}_{\mathrm{r}}$ measurements. DESI combines experimental features of both ESI (see section 2.7.6) and MALDI (see section 8.2.2) and a thorough understanding of both techniques is required to fully recognize the potential applications of DESI in the fields of biology, chemistry, forensics, and toxicology.

\subsubsection{Matrix Assisted Laser Desorption Ionization (MALDI)}

Matrix assisted laser desorption ionization (MALDI) was first described in 1985 by Karas et al. who employed the technique for the ionization of organic molecules while simultaneously studying the influence of the wavelength on laser desorption[268]. Since its introduction some 
twenty years ago, MALDI, along with ESI has become the heart of virtually all proteomic experiments due to its ability to provide the key tools essential for the analysis of proteins[138]. Although the exact mechanism of ionization and desorption during MALDI experiments remains debatable, it is widely accepted that the transfer of energy to an organic matrix causes rapid thermal heating of molecules and eventually leads to desorption of both matrix and analyte ions into the gas phase (Figure 40)[138].

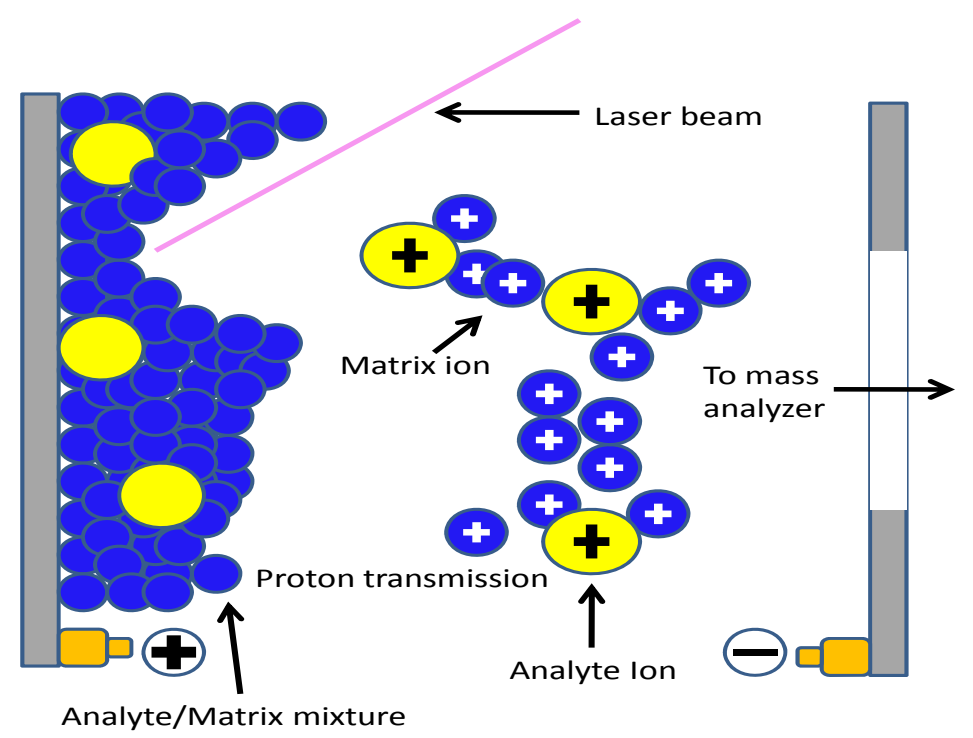

Figure 40: Schematic representation of MALDI ionization region (Adapted from www.magnet.fsu.edu/.../ionization_maldi.html).

While theories regarding the mechanistic pathway of MALDI remain largely divergent, significant convergence in one area has resulted in the widely accepted theory that MALDI ionization occurs via a two step process, involving initial matrix (primary) ionization followed by analyte (secondary) excitation and ionization. 
MALDI is achieved by co-crystallizing a sample with a thousand-fold excess (minimum) of an organic matrix on a metal target. A pulsed laser is then focused onto the surface of the matrix-analyte solid solution[269]. The organic matrix is chosen based on its ability to absorb energy at the wavelength corresponding to the laser being employed[270]. Frequency coupling between the incoming laser and the matrix chromaphore causes rapid vibrational excitation leading to localized disintegration of the solid solution. Laser ablative induced disintegration of the solid solution surface results in the ejection of 'clusters' composed of analyte molecules surrounded by matrix (primary) ions[271]. Following evaporation of matrix molecules, free gasphase analyte molecules occupy the ablation plume where they undergo ionization through proton transfer reactions with the photo-excited matrix molecules[271]. It is these analyte (secondary) ions which are then extracted and separated in the mass spectrometer based on their mass to charge ratios[272].

Considerable time and effort has been expended into the further development and application of MALDI, however, minimal progress has been made in the understanding of its mechanistic principles. Without a thorough mechanistic understanding of the processes behind MALDI analysts may be unequipped to handle the difficulties often associated with absolute sensitivity, variable response factors, range of applicability and reproducibility[273]. This lack of mechanistic understanding is evident in the literature by the predominately empirical approach toward MALDI research and utilization. While the empirical approach to MALDI continues to dominate in fields such as proteomics and biomolecular analysis, others have concentrated research efforts on further understanding experimental variables. One study aims to quantitatively predict or interpret observed mass spectra as a function of experimental variables such as, matrix choice, analyte physical and chemical properties, concentrations, preparation 
method, laser characteristics, local environment and ion extraction method, by drawing on the mechanistic principles behind the technique[273]. Studies exploring mechanistic theory and evaluating proposed models of MALDI will aid in its practical applications and provide a better understanding of factors capable of affecting relative and absolute signal intensities[273].

MALDI has quickly found notoriety as an analytical tool for the characterization of peptides and proteins. Coupled to the appropriate mass analyzer, MALDI allows for the accurate mass analysis of low-picomole amounts of biomolecules with molecular weights exceeding 100 $\mathrm{kDa}$. MALDI offers the inherent advantage of generating low charge state ions from samples containing appreciable levels of commonly encountered buffers and salts which can significantly affect ionization efficiencies when employing alternative techniques. The use of a pulsed laser during MALDI experiments results in the production of packets of ions rather than a continuous beam as in the case of ESI[138].

The mass spectra of ions generated by MALDI are typically measured using a time-offlight mass spectrometer. This is primarily due to the fact that time-of-flight mass analyzers are immediately compatible with the pulsed generation of high-energy ions and provide a theoretically unlimited mass range[270]. Each pulse of laser radiation generates packets of ions in a relatively short $(\approx 5 \mathrm{~cm})$ source region. An electrical potential contained in the source region then accelerates the ions to a fixed kinetic energy before allowing them to traverse the field-free drift region of the TOF mass spectrometer. Ions ultimately arrive at the detector following a drift time that is dependent on their mass. The drift time or TOF of each packet of MALDI generated ions is measured by an internal clock which is triggered by the laser pulse, making time-of-flight mass analysis perfectly suited to the pulsed ionization process utilized in MALDI [269, 270, 274]. 


\subsubsection{History and Background of Meconium Screening}

Screening techniques employed for the purpose of identifying drugs of abuse in meconium have largely utilized immunoassay analysis. The large majority of meconium studies published in the scientific literature have utilized either radioimmunoassay or enzyme immunoassay techniques. Immunoassays are based on an interaction between a drug (antigen) and specific antibody. When the drug of interest is recognized by the antibody, the subsequent interaction produces an antigen-antibody complex which is then separated from the remainder of the reaction mixture. The endpoint of the reaction, whether it be radioactivity in the case of radioimmunoassays or enzymatic activity in the case of enzyme immunoassays, is then measured and provides an indication of the quantity of drug present[39].

Radioimmunoassays (RIA) are performed by first generating a known quantity of radiolabeled antigen. This is normally achieved using radioactive isotopes of iodine attached to tyrosine. The radioactive labeled antigen then competes with the wild antigen (the drug of interest) for a limited number of binding sites in an antibody[39]. As the concentration of drug in the sample increases, the amount of bound radioactive antigen decreases due to fewer available binding sites in the antibody. The concentration of the drug can be estimated using a doseresponse curve generated by analyzing standards of known concentrations[39]. RIA has been widely employed for the detection of drugs of abuse in meconium and the vast majority of methods published prior to 1990 relied heavily in this technique[243]. The main drawback associated with RIA is the hazard and cost of preparing, handling, and disposing of the radioactive labeled antigen. RIA still finds widespread use in clinical laboratories, however, the majority of studies involving the detection of drugs of abuse in meconium published in the last fifteen years have utilized enzyme immunoassay. 
Enzyme linked immunosorbent assay (ELISA) is the most commonly employed enzyme immunoassay for various reasons including its inherent sensitivity, ability to handle multiple samples, and safe reagents. ELISA operates on the same principles as RIA in that the drug of interest competes with an enzyme labeled antigen for a limited number of binding sites in the antibody. Antigen labels are non-radioactive and are linked to an enzyme while the antibody is immobilized into a solid support[39]. The enzyme used to label the antigen is chosen based on its ability to produce a colored product from a colorless substrate. This color reaction between the enzyme and the substrate is the endpoint of the assay and can be used to estimate the concentration of drug in the sample. Due to the competitive binding nature of the assay, the concentration of the drug is inversely proportional to the concentration of the enzyme labeled antigen that is bound to the antibody[39].

Enzyme multiplied immunoassay technique (EMIT) also finds widespread use as a screening tool for drugs of abuse in biological specimens. EMIT is similar to ELISA in that the antigen is again linked to an enzyme however it is the activity of this enzyme that indicates the presence or absence of a drug class. The enzyme used to label the antigen is inactivated upon binding with the antibody meaning that enzymatic activity will be higher in the presence of the drug due to competitive binding mechanisms. The total amount of drug present in the sample is directly proportional to the enzymatic activity which is measured as the product of a reaction. Enzymatic activity is the endpoint of the assay and indicates the total amount of drug in the sample[39].

Unlike the National Institute on Drug Abuse protocol regulating urine testing, meconium analysis is not government regulated and many of the testing techniques have been directly adapted from pre-existing urine assays with the assumption that specific analysis procedures 
have no significant impact on results[275]. Due to the complex nature of the meconium matrix and the lack of research detailing its composition, the presence of endogenous compounds capable of generating false positives must be considered. False positive results arise when endogenous or exogenous components of the sample are erroneously recognized as the drug of interest by the testing technique. Recognition of the interfering compound as the drug of interest generates a positive result in the true absence of the drug. Specimens that screen positive should be subject to subsequent confirmation testing utilizing mass spectrometry where feasible.

The superior selectivity of mass spectrometry provides the analyst with structural information for the drug of interest, eliminating the possibility of false positives. Incorrect recognition of interferences as the drug of interest may be due to similarities in chemical structure or simply to the lack of selectivity afforded by most immunoassay techniques. Situations in which the drug of interest is present but not detected by the technique are referred to as false negative results and must also be considered when adapting pre-existing testing techniques to the analysis of complex matrices such as meconium. False negative results must also be considered when adapting a pre-existing extraction technique to a new matrix. Many of the extraction techniques employed for the confirmation testing of drugs in meconium have been directly adapted from pre-existing blood assays. Inappropriate or inefficient extraction of drugs from meconium can result in a negative confirmation result through analyte loss.

Although many studies involving the detection of drugs of abuse in meconium by way of immunoassay techniques appeared in the literature in the late 1980s and early 1990s, it was not until 1995 that the issue of false-positive and false-negative rates was addressed by Moore et al. from the US Drug Testing Laboratories in Chicago. Moore and coworkers investigated the rates of both false-negative confirmation results arising from inefficient extraction of drugs, and false- 
positive results arising from screen-only testing[275]. Experiments were aimed at determining whether differences exist between published screening procedures and to determine whether reporting screen-only data is reliable and acceptable. The authors compared three published procedures for the determination of abused drugs in meconium to determine the false-negative rates associated with various screening methods. Following this, additional experiments were performed with the aim of calculating the rate of false-positives by determining the actual number of immunoassay screen-positive samples which were subsequently confirmed negative by GC-MS. False-positive rates were investigated to assess the accuracy of screen-only results which are often the only results generated by hospital laboratories. The use of screen-only results for decision making is unethical and in the case of meconium analysis, can have devastating consequences not only for mothers, but also for children and other family members [275]. Although all negative specimens were correctly screened negative, results indicated that the three screening techniques differed substantially in their ability to detect the drugs of abuse in predetermined positive samples. Only $19.6 \%$ of the positive samples were identified using one method while the remaining two methods had success rates of $54.5 \%$, and $100 \%$. Results indicate that considerable time and effort is required during method development and optimization to minimize the potential for false-negative screen results. Experiments aimed at determining the rate of false-positives illustrated the importance of confirmation testing as only $53.3 \%$ of samples which screened positive were subsequently confirmed positive, indicating a false-positive rate of $46.7 \%[275]$. The greatest discrepancies between screen and confirmation data occurred with the amphetamine drug class in which only $25.7 \%$ of positive screens were confirmed positive by GC-MS. Of the 228 specimens that screened positive for cocaine metabolites, only $59.2 \%$ were confirmed positive. The opiate drug class had a similar false- 
positive rate with $56.7 \%$ of positive screens confirming positive[275]. To some degree, high false-positive results can be attributed to the presence of various over the counter (OTC) and prescription medications which contain cross-reacting substances, however, confirmation testing to distinguish these compounds from drugs of abuse is essential to make ethical and accurate decisions[275].

The suitability of immunoassay screening kits for the detection of drugs of abuse in meconium was also investigated by ElSohly and coworkers in 1999[243]. The authors aimed to develop and validate methods for meconium sample preparation prior to screening and confirmation testing while simultaneously evaluating the suitability of two different immunoassays for the purpose of screening for various drugs of abuse in meconium[243]. Experiments involved subjecting ninety five meconium specimens to the screening procedures and confirming any presumptive positives using GC-MS to determine the rate of false-positives. False-negative rates were also investigated by subjecting seventy meconium specimens for each drug class to confirmation analysis regardless of screening results. While immunoassay technologies did not generate any false-negative results, rates of false-positives were again alarming and ranged from $15 \%$ for opiates, to $100 \%$ for amphetamines[243]. The authors highlight the importance of carrying out GC-MS confirmation of positive screens prior to reporting results due to the high rate of false-positives.

Rates of false-positive results in authentic meconium specimens were also monitored at AIT Laboratories (Indianapolis, IN) for the cocaine and amphetamine drug classes. Following the implementation of UPLC-MS/MS methodologies for meconium confirmation analysis (see chapters $5 \& 7$ ), the rate of false-positives was monitored over an eight month period $(07 / 01 / 2008-03 / 01 / 2009)$. All specimens were screened using ELISA and the false-positive rate 
was determined by calculating the percentage of positive screens that were subsequently confirmed negative. During the eight month time period a total of 4036 meconium specimens were screened for amphetamines and cocaine. Of the 303 samples that screened positive for cocaine/benzoylecgonine, 71 were confirmed positive representing a false-positive rate of $76 \%$. Of the 138 samples that screened positive for amphetamines, only 37 were confirmed positive representing a false-positive rate of $73 \%$.

Based on results from the reported studies investigating the suitability of immunoassays for the purpose of screening meconium, it is evident that reporting screen-only results is both unethical and inaccurate due to the high rate of false-positive results. False-positive results not only complicate decision making processes for health care professionals, they also result in additional costs for the toxicology laboratory through unnecessary confirmation analysis. While the confirmation analysis is of extreme importance in the case of false-positives, such testing requires expensive reagents for suitable sample preparation and additional expenses associated with labor. Confirmation analysis is an expensive procedure for laboratories utilizing state of the art technologies such as LC/MS/MS for the purpose of providing enhanced sensitivity. Traditional confirmation techniques such as GC-MS require substantial sample preparation such as liquid-liquid or solid phase extraction of the drug from the meconium matrix, purification of the extract, and derivatization of the analyte, all of which require expensive chemical reagents. In addition to the cost of reagents, sample extraction and preparation prior to instrumental analysis can be very labor intensive consuming both time and labor costs for the toxicology laboratory.

False-positive results associated with immunoassays are inevitable due to the inferior selectivity associated with the technique and the complexity of the biological samples being analyzed. Laboratories employing confirmation testing for the purpose of unequivocally 
identifying the drug of interest recognize the potential for false-positives, however, the ability of immunoassays to screen out a large majority of negative samples in a cost effective manner somewhat offsets this disadvantage. High throughput production laboratories view immunoassay screening kits as a cheap, quick, reliable and effective way to screen biological samples for drugs of abuse, however, the potential for high rates of false-positive results means that laboratories must carefully monitor the time and cost associated with performing such assays. From a production standpoint, there is little value in dedicating resources to performing immunoassay screening tests that generate a large number of false-positive results requirin expensive confirmation testing.

While it is widely accepted that screen testing will never possess the selectivity of confirmation analysis, laboratories must continue to monitor the effectiveness of screening techniques with respect to their intended purpose. An immunoassay screen test generating a $100 \%$ false-positive rate is obviously not serving its purpose as an effective way to screen samples, and careful monitoring of the costs associated with avoidable confirmations relative to the effectiveness of the screen will allow the laboratory to assess the overall value of the technique.

Sample preparation prior to immunoassay analysis can be laborious depending on the specific screening technique, the analyte of interest and the biological matrix in which it exists. ElSohly et al. proposed a procedure for the preparation of meconium extracts for immunoassay analysis[243]. The authors investigated several procedures for the extraction of drugs from the meconium matrix prior to immunoassay analysis. Many of these methods were deemed to be inefficient, inconsistent, impractical, or generally problematic and therefore useless for meconium extractions[243]. Reduced sensitivity, maximal background signals leading to invalid 
results, low recoveries, and colored extracts unsuitable for analysis were some of the problems encountered throughout investigations into multiple sample preparation techniques. The final extraction method was developed based on the observations from previous trials and is outlined in Figure 41. 


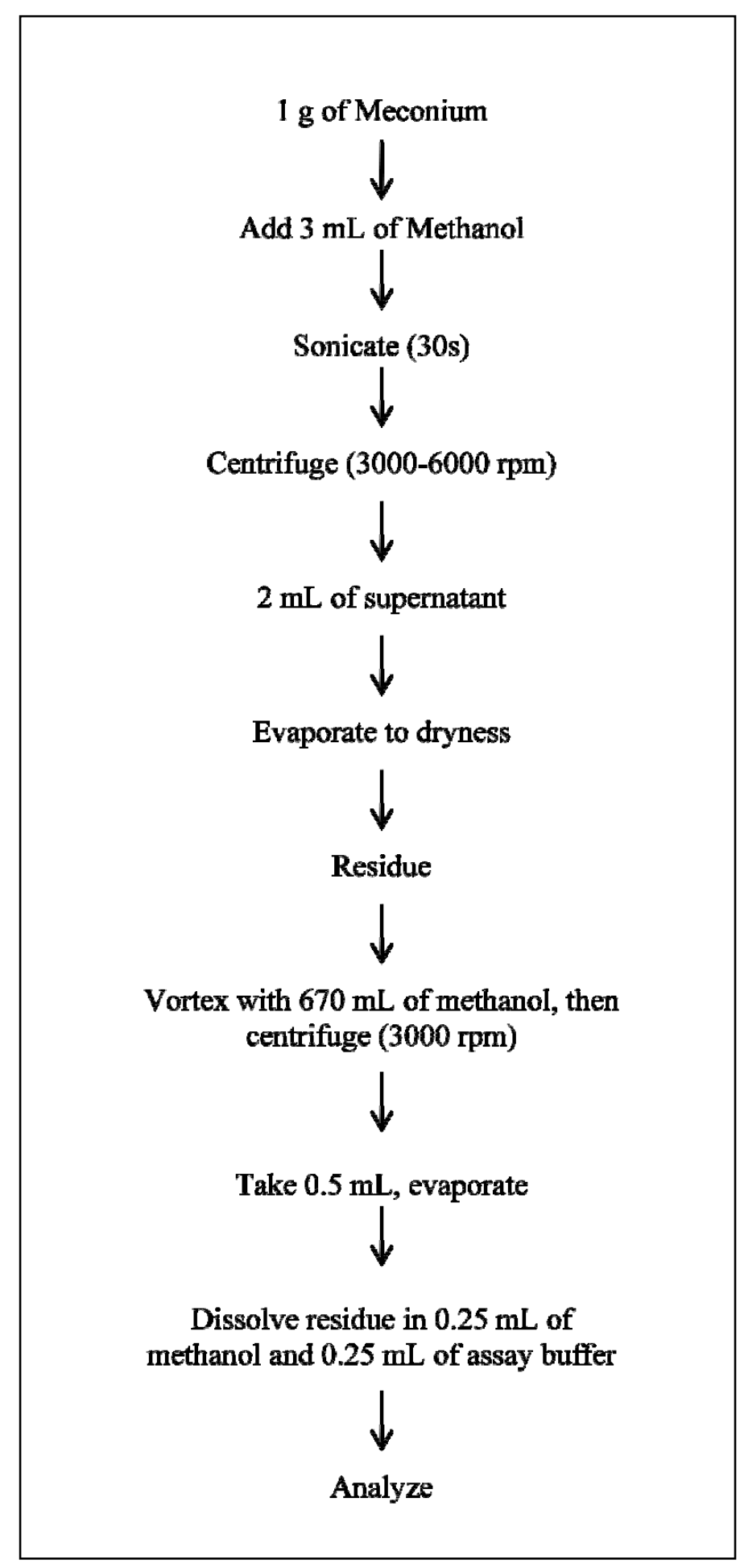

Figure 41: Proposed procedure for the preparation of meconium extracts for immunoassay analysis (Adapted from ElSohly et al., 1999).

The procedure proposed by ElSohly and coworkers for the preparation of meconium extracts for immunoassay analysis is laborious and a represents a significant sample work up. The 
performance of an immunoassay test requiring such extensive sample preparation should be monitored closely to ensure that unnecessary confirmations as a results of false-positives are minimal. The value of an immunoassay screening test which requires significant sample preparation and generates a large number of false-positives is arguably negligible, and investigations into alternate screening techniques more suited to the analysis at hand may prove beneficial for laboratories experiencing this problem.

Commercially available immunoassay screening kits are designed for the detection of drugs in blood and/or urine and care must be taken when adapting such methodologies to alternate matrices. Alternate toxicology samples such as amniotic fluid, tissue, fly larvae, and meconium are complex biological matrices rich in interfering species of both endogenous and exogenous origin. The suitability of immunoassay based testing for such specimens must be carefully evaluated as the potential for false-positive results will be much higher in the complex matrix. More alarming than the possibility of false-positive results which generate additional costs for the toxicology laboratory is the possibility of false-negative results.

False-negative results arise when screening kits are unable to detect a drug of interest, and can arise due to matrix complexity or sensitivity issues. False-negative results are a major concern for toxicology laboratories as testing is normally discontinued following a negative screen. In the case of a false-positive, subsequent confirmation testing utilizing mass spectrometry will indicate the true absence of the drug, however, in the case of a false-negative, there is no confirmation test. Following a negative screen, specimens are simply reported negative and discarded following a standard storage period. As a result, laboratories must ensure that screening techniques employed for the purpose of identifying drugs of abuse are suitable for the analysis at hand. While false-positive results generate additional costs for the laboratory 
through unnecessary confirmation testing, false-negatives mean that drug use goes undetected. In the case of meconium specimens, false-negative results mean that maternal drug use goes undetected and the drug-exposed child is denied the treatment and care that is normally provided following authoritative intervention in such cases.

Due to the increasing utilization of alternate toxicological specimens for the determination of drugs of abuse, the use of alternate screening techniques more suited to such complex biological matrices must be investigated. Immunoassay based technologies are likely to generate higher rates of false-positives when used for the analysis of more complex species due to the lack of selectivity associated with the technique. As the number of endogenous interfering species in the matrix increases, the accuracy of the immunoassay is likely to decrease as the inferior selectivity of the technique will be exploited to a greater extent than with traditional blood or urine based specimens. The use of mass spectrometry for screening purposes would alleviate many of the problems associated with immunoassay based techniques by affording inherent selectivity and eliminating the possibility of false-positive results.

\subsubsection{Mass Spectrometry as a Screening Tool}

Investigations into the use of mass spectrometry as a screening tool for drugs of abuse in biological specimens date back to the late 1970s. Thompson described one of the earliest GC-MS methodologies developed as a screening tool for the detection of anticonvulsant drugs in urine[276]. Sample preparation involved evaporating a small aliquot of urine (50-200 $\mu \mathrm{L})$, and permethylating the residue with methylsulfinylmethide carbanion and methyl iodide. Product mixtures were resolved using GC allowing the authors to identify drug metabolites, mono-, di-, and trisaccharides, and organic acids, including fatty acids and glucuronides based on mass spectral data. 
Wilson and coworkers described a GC-MS methodology employing chemical ionization mass fragmentography for the purposes of simultaneously identifying seven secondary and tertiary tricyclic antidepressants in plasma[277]. Sample preparation involved a one step liquidliquid extraction into hexane which was subsequently injected onto the chromatograph. The authors present the methodology as a suitable technique for monitoring therapeutic concentrations and for screening plasma samples in suspected overdose cases where the drug of abuse has not been identified.

In 1980, Dugal et al detailed the methodological aspects of a computerized system for the gas chromatographic screening and primary identification of several CNS stimulants and narcotic analgesics in human urine[278]. Sample preparation involved a relatively simple liquidliquid extraction followed by sample derivatization. Analytes were screened using selective nitrogen detection. The authors conclude that the methodology is suitable for the purposes of screening several drugs and their respective metabolites in a short amount of time.

Broad based screening methodologies utilizing gas chromatography coupled to one of several detection systems continued to appear in the literature in the years following this early work. The vast majority of methodologies employed mass spectrometry for the purpose of unequivocally identifying analytes based on EI fragmentation patterns and library matching. While GC-MS methodologies offer fast analysis times and mass spectrometric detection for the screening of acidic, basic, and neutral compounds, and sample preparation can prove laborious and reduce the overall effectiveness of the screening technique.

Due to the nature of the column packing in gas chromatographs, samples must be introduced onto the column in an organic solvent of minimal polarity. Many methodologies 
described in the literature satisfied this requirement by buffering the sample matrix with a base and extracting basic analytes into organic solvents suitable for direct injection. In addition, many compounds are too polar and/or do not possess sufficient volatility for direct GC-MS analysis. To alleviate such problems, many compounds must be chemically derivatized prior to GC-MS analysis. Sample derivatization is performed to decrease the polarity and/or increase the volatility of an analyte which does not naturally lend itself to analysis by GC-MS. The incompatibility of GC-MS with aqueous solvents combined with the need for chemical derivatization complicates sample preparation and means that significant sample preparation/clean up is required to simply screen the specimen. While sample preparation for broad based GC-MS screening can be laborious due to the nature of the chromatographic system, the advantage of obtaining mass spectral screening data often offsets this disadvantage. As a result, many laboratories employ GC-MS for the purpose of screening for general unknowns. Figure 42 describes a typical sample preparation procedure for a broad based GC-MS screen.

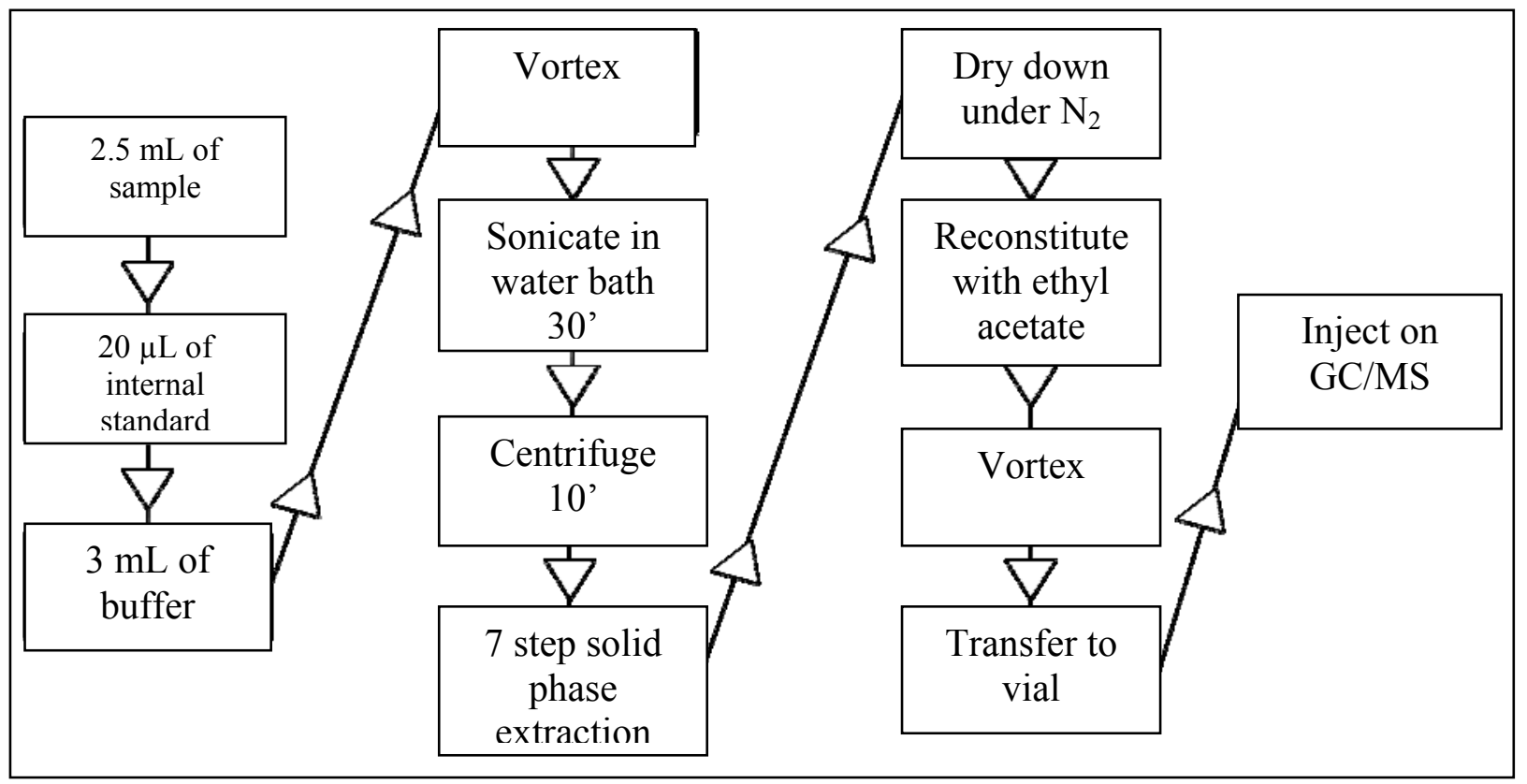

Figure 42: Extraction flow chart for a broad based GC-MS screen. 
Investigations into the use of LC-MS and LC-MS/MS for the purpose of general unknown screening began to appear in the scientific literature in the early 1980s. One of the earliest reports described the use of a triple quadrupole mass spectrometer for the rapid screening and confirmation of up to fifty analytes in a single sample of equine blood, serum, and urine in less than two minutes[279]. The enhanced sensitivity afforded by the tandem mass spectrometer enabled the authors to achieve detection limits in the $\mathrm{ng} / \mathrm{mL}$ to $\mu \mathrm{g} / \mathrm{mL}$ range for most analytes. The authors conclude that the use of LC-MS/MS technology for the purpose of screening provides a fast, sensitive technique for the accurate determination of selected drugs and metabolites in blood urine and serum.

Chromatographic resolution is not always necessary when employing mass spectrometry for the purpose of screening, provided the instrument possesses the capability to operate in SIM, SRM, or MRM mode. As a result, technologies such as GC/MS and LC/MS/MS have the capability to simultaneously screen for large numbers of drugs without the need for baseline resolution by monitoring selected ion transitions. Monitoring the presence or absence of compounds based on an ion trace rather than retention time means that rapid chromatographic separations are possible as extracted ion traces provide accurate qualitative data even in cases of chromatographic co-elution. Mass spectrometers coupled to liquid chromatographs offer additional advantages over GC/MS technologies as aqueous extracts of biological samples can be analyzed directly due to the column chemistry of the separation and the sophisticated nature of the LC/MS interface.

The ability of LC/MS and LC/MS/MS technologies to simultaneously screen for large numbers of drugs following minimal sample preparation and rapid LC separation was the driving force for the large number of methodologies appearing in the literature in the following years. 
The majority of methods described rapid, broad based screens for drugs of abuse in biological samples. In 1987, Straub and coworkers described the use of tandem mass spectrometric techniques for the purpose of rapid structural elucidation and metabolite quantification in complex sample matrices[280]. The authors report the ability of tandem mass spectrometers to serve as separation devices as well as tools for structural elucidation. Studies employed desorption ionization techniques and primary metabolites along with drug conjugates were identified using an appropriate combination of neutral loss and precursor ion scans[280]. Jones and colleagues from the facility of advanced instrumentation employed tandem mass spectrometry for the purpose of screening mercapturates in urine following ionization in the negative ion mode[281]. The authors employed neutral loss scanning to screen urine samples for the presence of mercapturates in the low nanogram per milliliter range. Identifications were made based on the simple daughter ion spectra of the deprotonated molecular ions. In the same year, Brzezinka and colleagues also employed the neutral loss scanning mode of a tandem mass spectrometer for the purpose of screening serum samples for the presence of barbiturates[282]. Subsequent confirmation testing and quantification was performed using MRM and limits of detection were reported to be better than one microgram per milliliter for most analytes.

Weinmann and Svoboda employed solid phase extraction followed by flow injection analysis (FIA) with ionspray-ionization and tandem mass spectrometry for the simultaneous and quantitative screening of illicit drugs in serum and urine[283]. Experiments were performed on an API 3000 triple quadrupole instrument and MS/MS analysis was performed by sequentially isolating the precursor ions of the analytes and their deuterated standards with subsequent fragmentation and monitoring of one fragment ion for each substance[283]. The authors conclude that the increased speed, sensitivity, selectivity, and accuracy afforded by the tandem 
mass spectrometer offers many advantages over traditional screening methodologies for the simultaneous determination of different drugs and metabolites in biological samples. Nordgren and co-authors evaluated the usefulness of LC-MS/MS for the purpose of screening urine samples for the designer amphetamines MDA, and MDMA[284]. Qualitative identifications were based on the detection of selected positive ions following APCI and studies were performed to assess the accuracy of the technique compared to immunochemical methodologies. Studies involved the analysis of 1000 clinical patient samples of which the LC-MS/MS methodology was able to identify four times as many positive samples as the immunochemical technique. The authors conclude that LC-MS/MS offers an attractive alternative to immunochemical techniques for drugs of abuse screening[284].

In 2004, Eichhorst and colleagues published one of the earliest studies aimed at directly comparing LC-MS/MS with more traditional screening techniques such as GC-MS and ELISA for the purpose of detecting methylphenidate in urine samples[285]. The use of LC-MS/MS for screening urine samples was investigated in the hope of replacing less reliable, more expensive, and time consuming screening techniques such as GC/MS with a combined one-step screening and confirmation LC-MS/MS method. Implementation of the rapid LC-MS/MS method enabled the authors to achieve enhanced sensitivity, selectivity and reliability while simultaneously reducing the cost per sample.

In 2005, Mueller and co-workers described a broad based multi-target screening (MTS) procedure for the detection of over 300 forensically relevant drugs in blood and urine using a hybrid triple-quadrupole linear ion trap mass spectrometer (QTrap)[197]. This was one of the earliest studies employing the various scanning capabilities of a tandem mass spectrometer for the purpose of screening such a large number of drugs in a single LC-MS/MS run. A multiple 
reaction monitoring $(\mathrm{MRM})$ scan and an enhanced product ion (EPI) scan were performed in an information-dependant acquisition (IDA) experiment. The MRM survey scan contained 298 transitions for the 301 selected substances and three deuterated internal standards. In order to perform the EPI scan, Q3 was used as a linear ion trap to scan product ions. Drug identification was carried out by a library search with a newly developed MS/MS library based on EPI spectra[197]. Authors conclude that the developed methodology is useful for the rapid detection or exclusion of over 300 drugs in a blood, plasma, or serum extract. Limitations to the methodology include the cycle time required to perform both MRM and EPI experiments and multiple collision energies and the limited number of data points obtained across chromatographic peaks with $5 \mathrm{~ms}$ dwell times.

Nordgren and coworkers also employed a triple quadrupole mass spectrometer to simultaneously screen for 23 analytes in urine samples by monitoring the presence or absence of a single ion transition in the multiple reaction monitoring mode[286]. In a study of 3000 human urine samples, the methodology correctly identified the presence of at least one substance $65 \%$ of the time. Authors conclude that while the LC-MS/MS methodology offers a sensitive, robust alternative to more traditional screening techniques, future studies should consider incorporating additional ion transitions into the criteria for a positive result to decrease the number of false positive results obtained when monitoring only a single transition. Allen and coworkers investigated the usefulness of LC-MS/MS for the purpose of screening oral fluid samples for opiates, cocaine, methadone, and benzodiazepines and directly compared the method with the pre-existing ELISA method[287]. Individual analytes were again identified using multiple reaction monitoring and analysis of 72 patient samples indicated that the LC-MS/MS method compares favorably with the more traditional immunoassay based techniques. The authors 
conclude that the tandem mass spectrometric method offers a more flexible, selective and sensitive alternative for screening oral fluid samples than ELISA due to the large number of individual drugs and metabolites that can be detected from a single sample injection.

In the years to follow, several reports appeared in the literature describing the use of tandem mass spectrometers; mainly triple quadrupoles and quadrupole - ion trap hybrids (QTrap), for the purpose of screening large numbers of drugs and metabolites in biological specimens[168, 170,183, 288-291]. Several research groups investigated the applications of exact mass measurements for the purposes of forensic drug screening [292-294]. Studies involved the use of time-of-flight (TOF) mass spectrometry coupled with either LC or UPLC for the purpose of measuring exact mass. While the exact mass measuring capabilities of TOF mass spectrometry have long been utilized in new drug discovery and ADME studies, several early reports describing the use of TOF for forensic drug screening indicate the widespread applicability of the technique for general unknown screening. Time of flight mass spectrometry provides high resolution exact mass measurements while minimizing both sample preparation time and cost of analysis when compared to GC/MS or ELISA technologies. Development of analyte libraries based on exact mass and retention time provides the analyst with a powerful technique for the purpose of general unknown screening. Screening techniques based on triple quadrupole technologies such as TQDs or QTraps only possess the capability to screen for analytes which exist in a predetermined MRM library. This is due to the fact that MRM studies must first be performed on standard materials to identify characteristic fragmentation patterns suitable for analyte identification. Once the information has been experimentally determined, parameters can be incorporated into the pre-existing MS/MS method to screen for the new analyte. Addition of new analytes to pre-existing TQD or QTrap methodologies directly affects 
sensitivity as dwell times must be minimized to obtain sufficient data points across chromatographic peaks. While screening technologies based on TOF mass spectrometry may also utilize analyte library searches for the purpose of analyte identification, additional analytes which do not exist in the library may be identified based on exact mass. While analyte identifications based on LC-TOF or UPLC-TOF are generally made based on a combination of retention time and exact mass, qualitative data based solely on exact mass can be extracted postrun from data which has already been analyzed.

Over the past twenty years, the potential applications of mass spectrometry as a screening tool have been investigated by many research groups. While the vast majority of methods have utilized triple quadrupole technologies or hybrid tandem mass spectrometers, recent studies has paved inroads into the use of alternate mass spectrometric methods requiring minimal sample preparation and little, if any, chromatographic separation. Mass spectrometric techniques requiring minimal sample preparation and no chromatographic separation will undoubtedly be the subject of rigorous future research efforts as the potential to reduce analysis time and increase the number of samples screened per unit time offers many advantages for high throughput toxicology laboratories.

\subsubsection{Principles of Desorption Electrospray Ionization (DESI)}

Desorption electrospray ionization allows ambient sampling for mass spectrometric analysis by using an electrospray of aqueous droplets which act to desorb analytes from a surface of interest. Analyte leaves the surface of interest entrained in small, progeny droplets (solvated) which subsequently evaporate resulting in analyte ion production. Desorbed gas-phase ions are then transferred to a mass spectrometer through an atmospheric pressure ion-transfer tube[263]. DESI is a multistep process which is initiated by producing primary charged droplets through an 
electrospray source. Primary droplets are then projected toward the surface of interest where they collide and produce smaller droplets through collision-induced breakup. Secondary charged droplets, which are produced during this interaction, are then transported, along with ablated materials and free ions, through an atmospheric pressure interface and into a mass spectrometer (Figure 39)[295].

DESI eliminates the need to introduce the sample into a region of vacuum or an inaccessible region closely coupled to a vacuum system meaning that the sample can be moved continuously or reoriented in space while collecting mass spectral data[263]. As previously mentioned, DESI experiments combine features of ESI with those of desorption ionization methods such as MALDI. An electrospray emitter, similar to those employed in traditional ESI experiments, is used to generate gas phase solvent ions, ionic clusters, and charged microdroplets which are directed as the surface of the sample. Several kilovolts of electrical charge are applied to the spray solution and pneumatic nebulization is used to assist with solvent desolvation. Electrosprayed microdroplets of the aqueous spray solution, normally containing additives which ensure maximal analyte ionization, act as projectiles and subsequently generate gas-phase ions when directed at the surface of interest through electrostatic and pneumatically assisted desorption mechanisms[263, 264]. The interaction of the spray with the surface of interest results in the ionization of analytes present on the sample exterior.

Analyte ions are then transported from ambient pressure conditions into a standard atmospheric pressure interface mass spectrometer[295]. The interrogating solution often consists of a water-alcohol mixture but may be modified with reagents such as acids or bases to enhance analyte ionization[295]. This spray solution is then electrohydrodynamically nebulized and directed at the surface of interest[295]. Unlike electrospray ionization (ESI) where the sample is 
dissolved in the spray solvent, analytes to be analyzed using DESI are present in solid materials or liquids which have been deposited onto suitable surfaces allowing for direct analysis without the need for extensive sample preparation[295].

The DESI spray solvent is an extremely important variable which can significantly affect the intensity and stability of analyte signal. Spray solvent composition requires careful consideration during method development as analytes must be somewhat soluble in the spray and its composition must be such that adequate droplet desorption can occur[296, 297]. Manicke and coworkers investigated the effect of spray solvent composition on signal intensity and stability by directing various sprays at a sample surface and monitoring the resultant ion current for the molecular ion. The authors directly compared five spray solvents comprised of different proportions of water and methanol[298]. The authors discovered that signal decay occurred more slowly with time as the proportion of water in the spray solvent increased. The increased rate of signal decay when using higher proportions of organic solvent can be attributed to the higher solubility of the analyte in the spray. In the presence of increased methanol content, more analyte is likely to be soluble in the spray leading to more efficient desorption from the surface and maximum ion signal. Increased sample solubility in the methanol rich solvent leads to maximum signal intensity at early time points with subsequent large decreases in signal due to depleted analyte concentrations at the exterior of the sample[298]. Maximum signal intensities were not significantly different for spray solvents consisting of $100 \%$ methanol, $75 \%$ methanol, and $50 \%$ methanol suggesting that additional factor others than analyte solubility are responsible for maximal desorption from the sample surface. This is in fact the case as increases in the organic component of the spray causes changes in solvent properties which work to negate the effect of increased analyte solubility. An example of these phenomena is the effect which increasing 
organic solvent has on spot size which represents the area of analyte that is sampled. As the concentration of methanol increases, the spot size and therefore the area of analyte sampled decreases due to the lower surface tension of the liquid. Experiments also indicate that when the amount of water in the spray solvent exceeds $50 \%$ there is a significant decrease in the maximum signal intensity. This is consistent with the theory that the analyte will be less soluble in the aqueous spray, however, poor droplet desorption due to unfavorable viscosity may also contribute to decreased signal intensity[298]. The authors conclude that a 1:1 methanol/water (vol/vol) solution typically provided the best combination of signal intensity and stability for the analytes of interest[298].

The exact composition of the solvent spray can be varied significantly depending on the analytical application of the experiment. Spray solvents consisting of high water content are often employed for $\mathrm{MS}^{\mathrm{n}}$ experiments which require more stable, longer lasting signal to complete mass spectral data acquisition. Similarly, high contents of methanol may be incorporated into the spray solvent for DESI experiments requiring high spatial resolution, such as molecular imaging. Spray solvents with higher fractions of methanol are more suited to such analyses as the high organic content reduces the spot size and provides greater spatial resolution[298]. Physical characteristics of the spray have also been investigated using phase Doppler anemometry (PDA) and experiments indicate that droplet sizes average 2-4 $\mu \mathrm{m}$, and at a distance of $2 \mathrm{~mm}$ from the spray source, velocities range between 100-200 $\mathrm{ms}^{-1}[295,297]$.

Venter and coworkers employed PDA for the purpose of determining the size and velocities of droplets involved in DESI experiments to further understand the likely ionization mechanisms behind the technique[295]. In contrast to desorption techniques which involve the production and acceleration of particles in the vacuum region of a mass spectrometer, such 
massive cluster bombardment (MCI), droplet impact/secondary ion mass spectrometry (EDI), and secondary ion mass spectrometry (SIMS), DESI occurs at atmospheric pressure. Ionization mechanisms are therefore likely to be very different to vacuum techniques as fast moving droplets will be slowed by aerodynamic drag forces due to their interaction with surrounding gas molecules[295]. It is for this same reason that DESI if finding an increasing number of applications in the forensic and clinical toxicology laboratory.

Samples analyzed by DESI are done so in the open environment of the laboratory which enables easy access during analysis for manipulation or higher throughput. Samples are also analyzed directly with little to no sample preparation, further alleviating problems associated with sample preparation for traditional instrumental analysis. While investigating the physical properties of droplets generated in DESI experiments, Venter and coworkers note that the kinetic energies per impacting water molecule are quite low and indicate that sputtering through momentum transfer during collisions or ionization via alternate electronic processes is unlikely[295]. The authors evaluate the validity of several proposed ionization mechanisms including droplet pick-up, chemical sputtering, evaporation followed by gas-phase ionization, and shockwave ionization by measuring the size and velocities of both inbound electrosprayed droplets and droplets leaving the sample exterior. Experimental evidence acquired throughout the study indicates that the droplet pick-up model may be the major process leading to the ionization of analytes during DESI experiments. The droplet pick-up model is believed to take place during the brief contact time that exists when impinging droplets collide with the sample surfaces and analytes are extracted into the departing droplet or droplet fragments. Following the salvation of condensed phase analytes by impinging droplets, typical electrospray ionization processes are thought to occur[295]. 
The DESI ion source is comprised of two main parts, the sprayer assembly and the surface assembly which are both mounted on the base of the ion source (Figure 43).

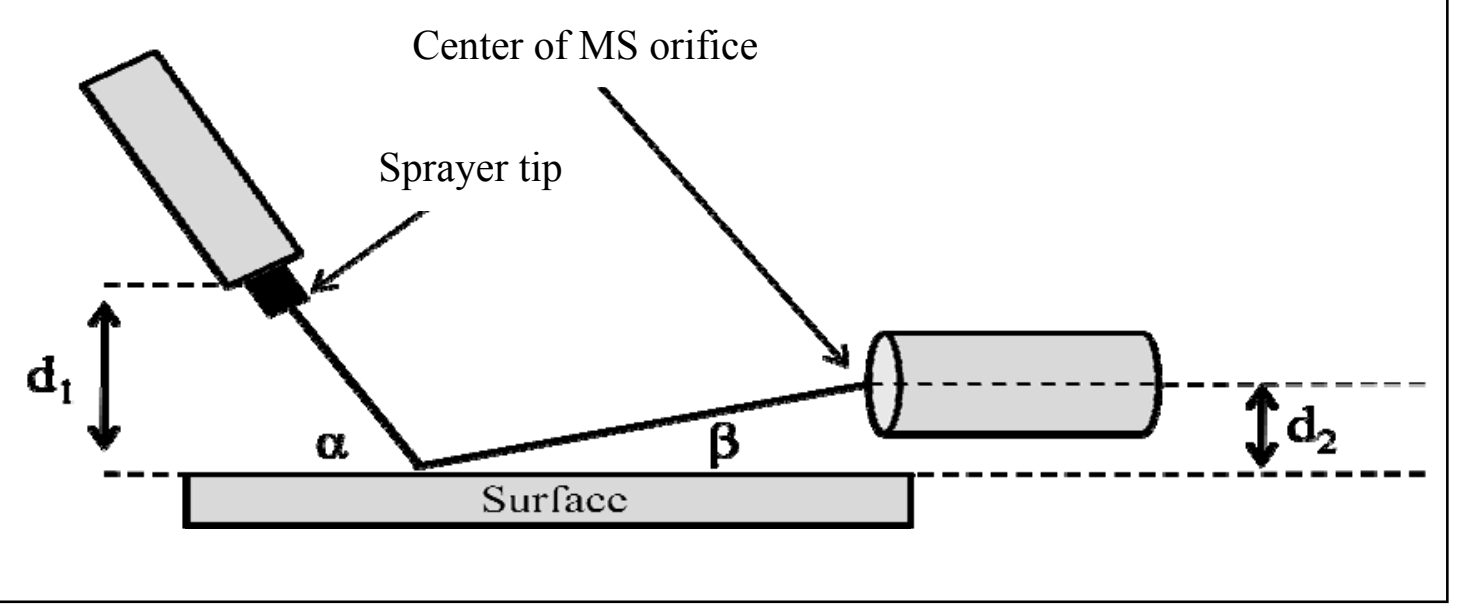

Figure 43: Schematic representation of the DESI ion source illustrating geometric parameters $\alpha$ (incident angle), $\beta$ (collection angle), d1 (tip-to-surface distance), and d2 (MS inlet-to-surface distance) (Adapted from Takats et al. 2005).

The sprayer is mounted to, and controlled from, a vertical rotating stage which is mounted onto a three dimensional linear moving stage. Linear motion is used to modify the sprayer-to-MS or sprayer-to-surface distance and to compensate for the different angles at which the sprayer is used[264].

Parameters associated with the geometry of the ion source, the solvent spray, and the sample surface, all affect the analytical performance of DESI and each require consideration and optimization during method development. Of greatest importance are the geometric parameters $\left(\alpha, \beta, \mathrm{d}_{1}, \mathrm{~d}_{2} ;\right.$ Fig 45$)$, gas and liquid flow rates, voltages, solvent composition, deposition solvent, and surface composition, temperature, and potential[264]. The geometric parameters, $\alpha$, and $\mathrm{d}_{1}$ have a direct effect on the efficiency of the ionization process, while parameters, $\beta$ and $\mathrm{d}_{2}$ have important effects of the collection efficiency, and therefore the sensitivity of the method. Cooks and coworkers indicate that the optimal settings for $\beta$ and $\mathrm{d}_{2}$ are generally $5-10^{\circ}$ and $0-2 \mathrm{~mm}$, 
respectively[264]. Generally, low $\mathrm{d}_{1}$ values are preferred and are normally combined with large $\alpha$ values, although a dramatic increase in chemical noise is observed when $\alpha$ exceed $80^{\circ}$ [264].

The design of DESI instruments is complicated by the challenge of providing efficient ion transfer from the ion source to the fore vacuum region of the mass spectrometer without modifying the pumping capacity of the vacuum system[264]. Metal capillaries are most widely employed for the transfer of ions to the mass spectrometer as capillaries containing insulators can experience significant charge buildup on the inner surface resulting in poor ion transmission. While metal capillaries do not experience this same phenomenon, extensive neutralization of analyte ions can occur which leads to poor transmission efficiencies.

Spectral characteristics of DESI are very similar to those of ESI in that spectra feature multiply charged ions, adducts, complexes, and abundant protonated molecular ions. Strong similarities between the two techniques are attributed to the similarities in the latter stages of ion formation. The drop pick-up ionization mechanism thought to occur in DESI experiments assumes that following analyte solvation by impinging droplets, analyte is incorporated into droplets and ionization occurs via traditional ESI mechanisms involving analyte-containing charged droplets[264].

\subsection{Experimental}

\subsubsection{Chemicals and Reagents}

All drug standards and deuterated internal standards $(1 \mathrm{mg} / \mathrm{mL}$ in methanol) were obtained from Cerilliant (Round Rock, TX). ISOLUTE ${ }^{\circledR}$ HM-N supported liquid-liquid extraction columns were purchased from Biotage (Charlottesville, VA). All solvents were HPLC 
grade and obtained from Fisher Scientific (Pittsburgh PA). All DESI experiments were performed using Omni Slides ${ }^{\mathrm{TM}}$ from Prosolia (Indianapolis, IN).

\subsubsection{Electrospray Solvent Preparation}

The spray solvent used for all experiments was an acetonitrile/water (80:20) mixture containing $0.1 \%(\mathrm{v} / \mathrm{v})$ formic acid.

\subsubsection{DESI Source and Mass Spectrometry Conditions}

All experiments were performed on the Thermo TSQ Quantum Discovery MAX triple quadrupole mass spectrometer equipped with the Omni Spray ${ }^{\circledR}$ ion source (Prosolia, Inc., Indianapolis, IN). Omni Spray® ion source operating parameters were as follows: Nitrogen was used as the nebulizing gas and was applied to the spray head at a regulated pressure of $125 \mathrm{psi}$. The solvent was sprayed under the influence of $5 \mathrm{kV}$ at a flow rate of $5 \mu \mathrm{L} / \mathrm{min}$. The gas jet was composed of electrosprayed aqueous microdroplets, and free gas-phase ions were directed onto the surface at an incident angle of $55^{\circ}$ to the normal. The vertical distance from the end of the solvent capillary to the top of the disposable surface was $2 \mathrm{~mm}$. The inlet of the mass spectrometer was maintained at a temperature of $300^{\circ} \mathrm{C}$. Total analysis time for each sample was approximately 13 seconds with a surface scan rate of $200 \mu \mathrm{m} / \mathrm{s}$.

\subsubsection{Sample Preparation}

All samples were spotted directly onto Omni Slides ${ }^{\mathrm{TM}}$ using a $2 \mu \mathrm{L}$ Eppendorf pipette.

Samples were left to dry under ambient conditions and analyzed directly using the DESI source.

\subsubsection{DESI Analysis of Standards}

Preliminary DESI experiments involved the analysis of amphetamine, methamphetamine, cocaine, benzoylecgonine, morphine, codeine, and hydromorphone standards $(1 \mathrm{mg} / \mathrm{mL}$ in 
methanol) for the purpose of developing and optimizing mass spectrometric conditions. The DESI-MS/MS instrument used for all experiments is analogous to the triple quadrupole instrument described previously except that the electrospray ionization source is replaced by the DESI source which allows for ambient sampling. Precursor ions for MRM experiments are first generated by the DESI source before entering the vacuum region of the triple quadrupole mass spectrometer where they undergo collision induced dissociation in Q2 while Q1 and Q3 are used as mass filters. In order to develop and optimize the MS/MS method, drug standards $(1 \mathrm{mg} / \mathrm{mL}$ in methanol) were spotted onto Omni Slides ${ }^{\mathrm{TM}}$ and analyzed directly. These preliminary studies are analogous to infusion experiments using the ESI-MS/MS in which the collision gas is turned off to allow the precursor ions generated by the DESI source to reach the detector and produce a recordable signal. Precursor ions for all seven analytes were protonated molecular ions generated by the soft DESI technique (Table 55).

Following identification of the molecular ion signal, an auto tune was completed for each analyte which involved adjusting collision energies to maximize the signal for the product ions generated in the collision cell. The mass transition from the protonated molecular ion $[\mathrm{M}+\mathrm{H}]^{+}$to the most abundant product ion was designated the qualifying ion transition and was used for all positive identifications (Table 55). Following auto tuning of each analyte, the optimized parameters were used to construct the MS/MS method which was then used to acquire data in the MRM mode. Table 55 reports the mass transitions, collision energies and tube lens voltages for each of the seven analytes. 
Table 55: Optimized MS/MS parameter employed for all DESI experiments.

\begin{tabular}{|l|c|c|c|c|}
\hline \multicolumn{1}{|c|}{ Analyte } & Parent mass $(\mathrm{m} / \mathrm{z})$ & Product ion $(\mathrm{m} / \mathrm{z})$ & Collision energy (V) & Tube lens (V) \\
\hline Amphetamine & 136 & 91 & 26 & 82 \\
\hline Methamphetamine & 150 & 91 & 22 & 81 \\
\hline Cocaine & 304 & 182 & 30 & 128 \\
\hline Benzoylecgonine & 290 & 168 & 79 & 118 \\
\hline Codeine & 300 & 165 & 30 & 128 \\
\hline Morphine & 286 & 152 & 20 & 124 \\
\hline Hydromorphone & 286 & 185 & 24 & 134 \\
\hline
\end{tabular}

Figure 44 shows the mass spectral responses for all seven analytes which were spotted in consecutive channels of the Omni Slide ${ }^{\mathrm{TM}}$.

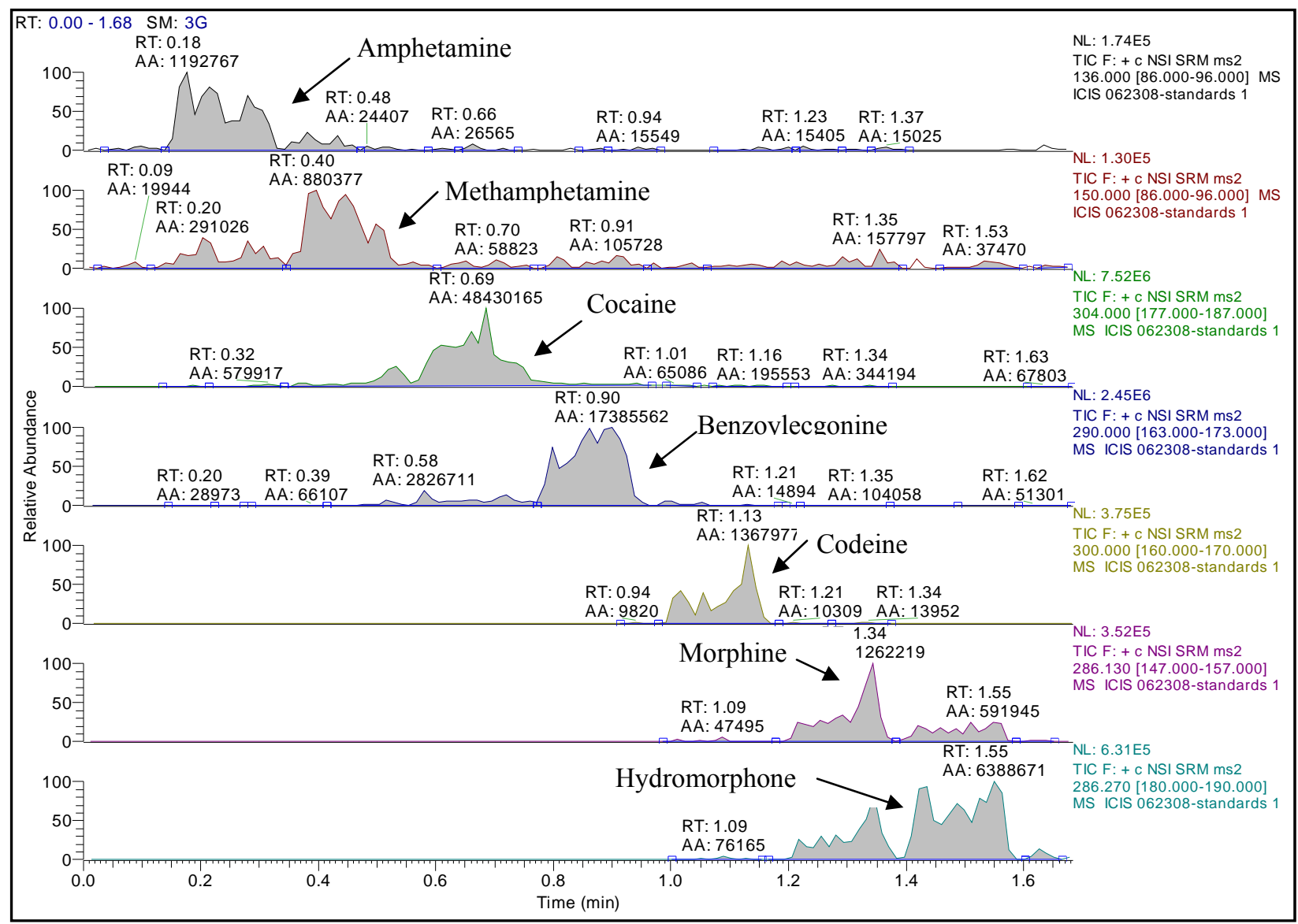

Figure 44: Mass spectral responses for all seven analytes using DESI-MS/MS. 
In order to determine the suitability of the DESI-MS/MS method for the analysis of authentic meconium specimens, several spiked samples were prepared and analyzed.

\subsubsection{Direct Analysis of Spiked Meconium Specimens}

Several meconium specimens which had undergone no sample preparation other than the initial homogenization step were spiked with appropriate analytes and analyzed directly using the DESI-MS/MS method. Experiments aimed to investigate the suitability of the DESI-MS/MS method for the direct analysis of meconium specimens having undergone minimal sample preparation.

\subsubsection{Sample Preparation}

Meconium specimens which had previously screened negative at AIT Laboratories (Indianapolis, IN) were spiked with varying amounts of analyte to give concentrations corresponding to the top point of calibration curves used in previous ESI-MS/MS experiments (Table 56). Concentrations were chosen arbitrarily for the purpose of evaluating the sensitivity of the DESI-MS/MS methodology. Prior to spiking, sample preparation of the meconium specimens was limited to the initial homogenization process required for immunoassay screening. Analytes of interest were then spiked directly into the homogenate at appropriate concentrations (Table 56).

Table 56: Analyte concentrations chosen for initial DESI-MS/MS experiments.

\begin{tabular}{|c|c|c|}
\hline Sample & Analytes & Concentration (ng/mL) \\
\hline 1 & morphine, codeine, hydromorphone & 500 \\
\hline 2 & amphetamine, methamphetamine & 500 \\
\hline 3 & cocaine, benzoylecgonine & 250 \\
\hline 4 & blank & $\mathrm{n} / \mathrm{a}$ \\
\hline
\end{tabular}


Negative meconium specimens $(1 \mathrm{~mL})$ were spiked with appropriate volumes of analyte, vortexed and spotted directly onto Omni Slides ${ }^{\mathrm{TM}}$. Samples were left to dry under ambient conditions and analyzed directly.

\subsubsection{Preliminary Results}

Extremely poor analyte signals were observed for all analytes prepared in the blank meconium matrix and analyzed directly (Figure 45).

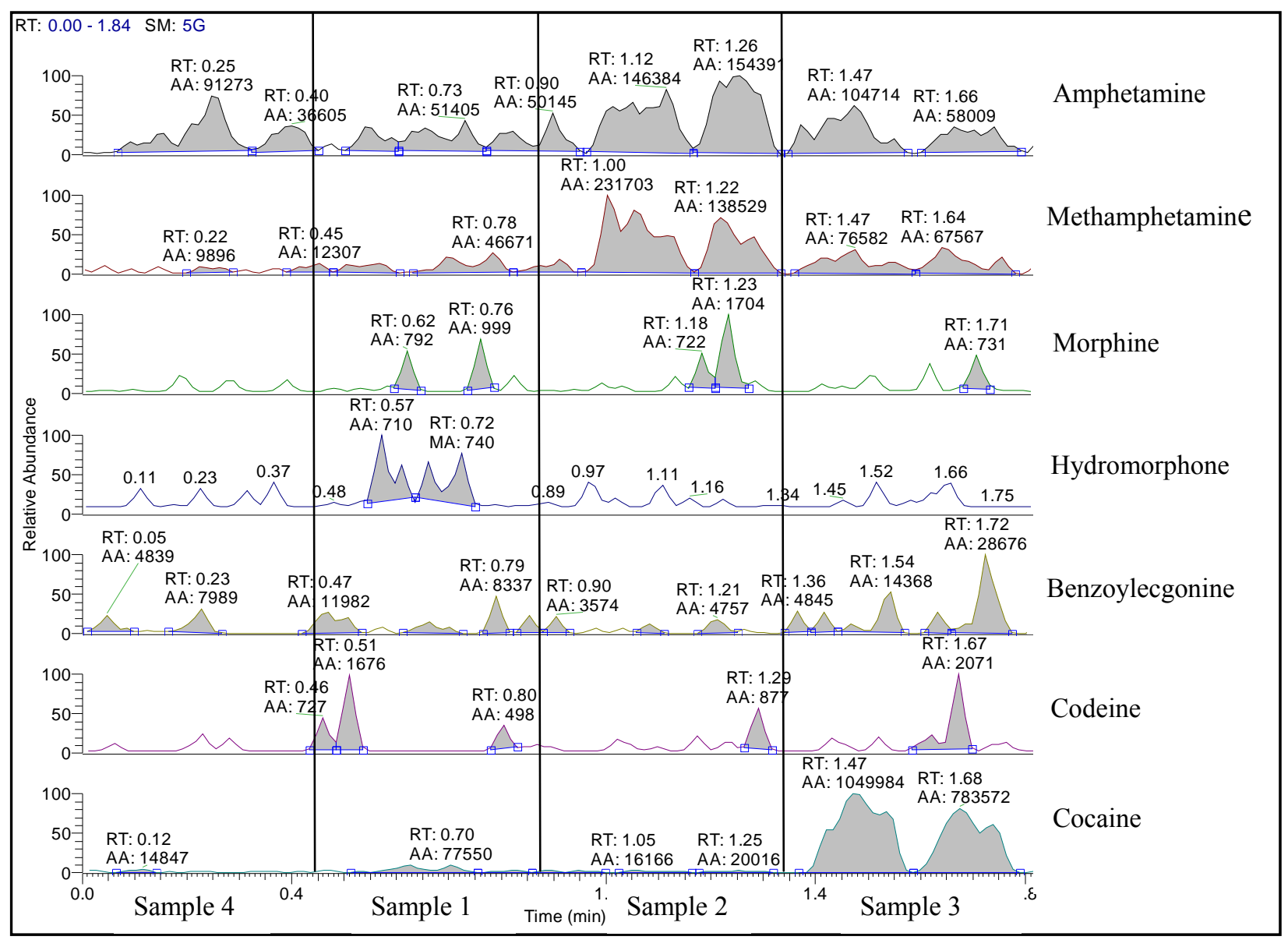

Figure 45: Analyte signals following direct analysis of spiked meconium samples.

Preliminary experiments were aimed at evaluating the usefulness of DESI-MS/MS for the purpose of screening meconium specimens which had undergone little to no sample pre- 
treatment. While the use of mass spectrometry for screening offers several advantages over traditional techniques, sample pre-treatment must be kept minimal in order for the technique to prove both time and cost effective. Poor signal intensities (Figure 45) indicated that significant matrix effects were likely suppressing mass spectral signal and that further sample clean up would be required to achieve the desired screening sensitivity. In order to evaluate the matrix effect on signal intensity, DESI spectra of neat drug standards were directly compared to samples prepared in blank meconium (Figures 46-48) at the same concentration. Experiments were performed by directly spotting neat drug standards on the first four channels of the Omni Slide ${ }^{\mathrm{TM}}$ and the meconium matrix containing the same concentration of analyte on the last four channels. By comparing analyte signal intensities from the neat drug standards relative to the spiked meconium specimens, the degree of signal suppression due to matrix effects was more apparent. 


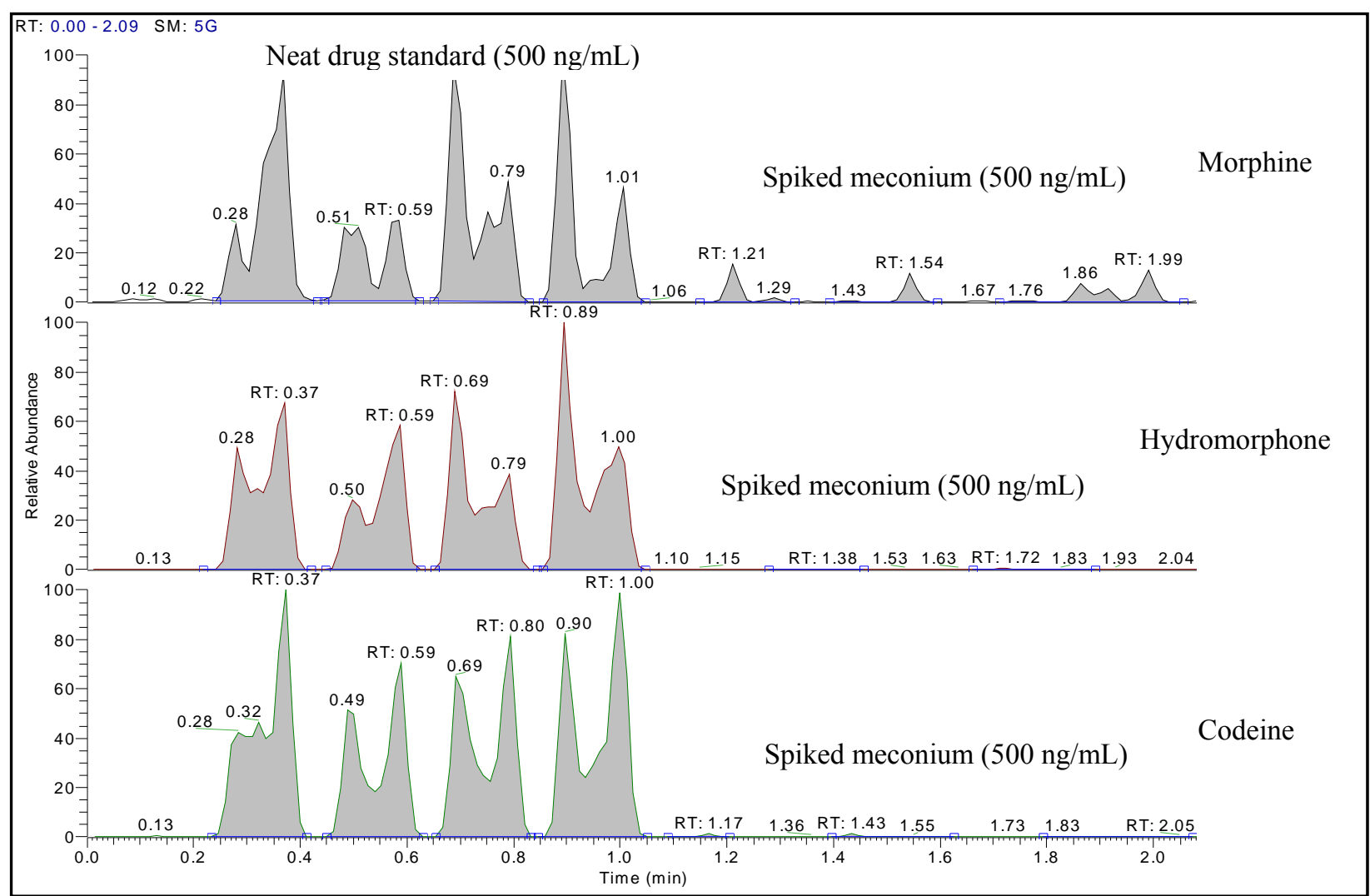

Figure 46: Effect of meconium matrix on morphine, hydromorphone, and codeine signal. Mass spectral signals for neat drug standards prepared at $500 \mathrm{ng} / \mathrm{mL}$ can be seen in channels 1-4 while analyte signals for spiked meconium standards containing $500 \mathrm{ng} / \mathrm{mL}$ of each analyte can be seen in channels 5-8. 


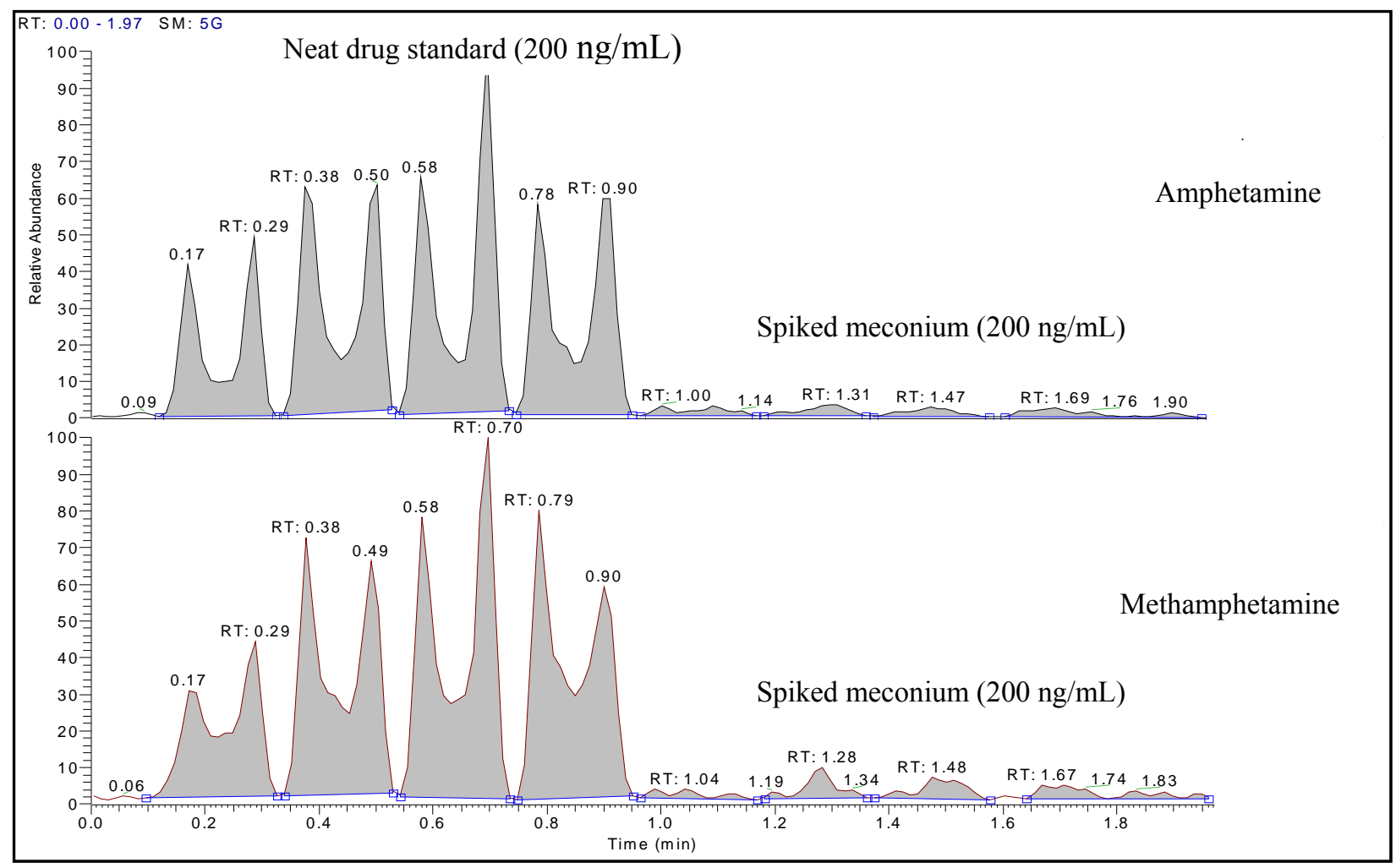

Figure 47: Effect of meconium matrix on amphetamine and methamphetamine signal. Mass spectral signals for neat drug standards prepared at $200 \mathrm{ng} / \mathrm{mL}$ can be seen in channels 1-4 while analyte signals for spiked meconium standards containing $200 \mathrm{ng} / \mathrm{mL}$ of each analyte can be seen in channels 5-8. 


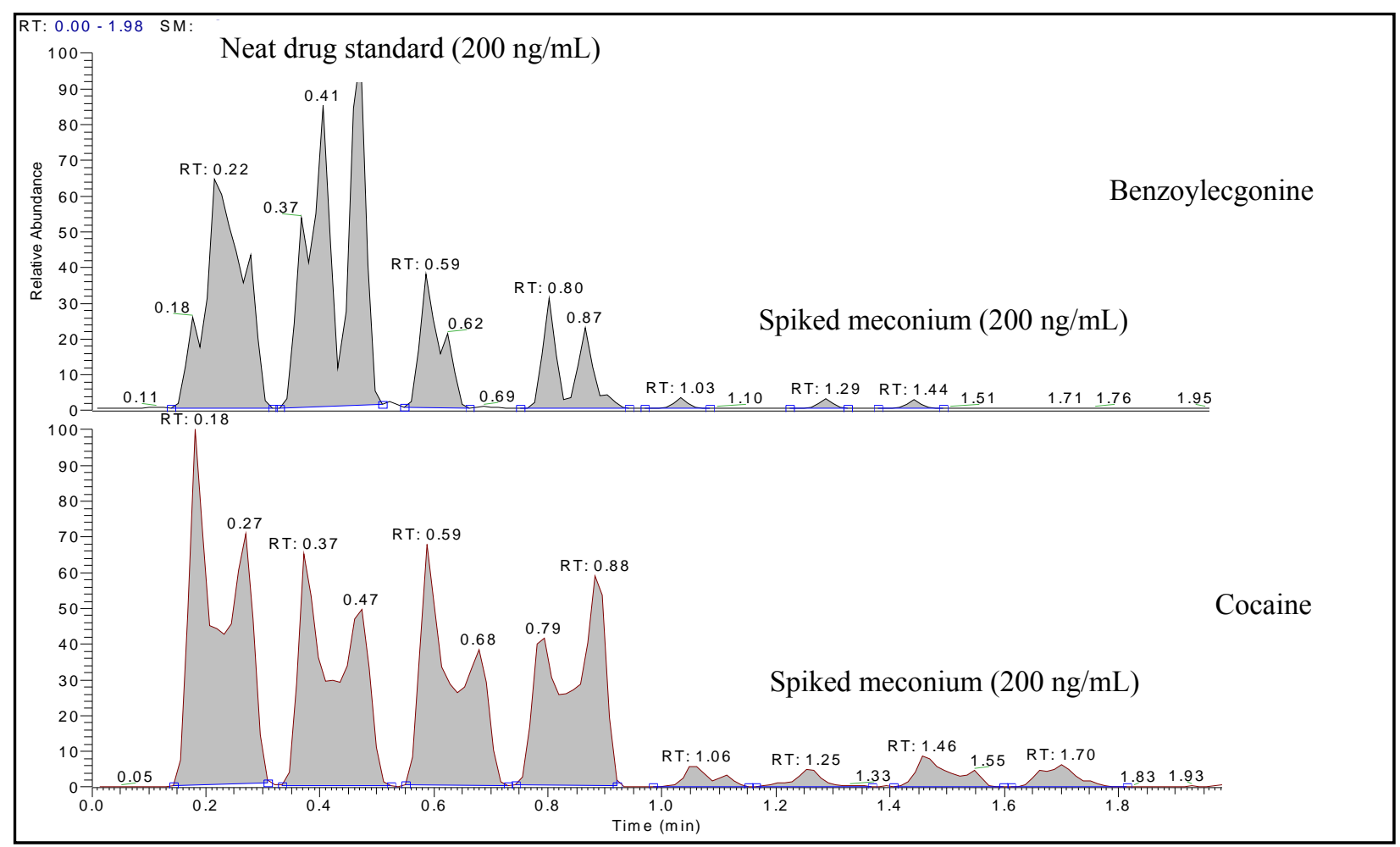

Figure 48: Effect of meconium matrix on cocaine and benzoylecgonine signal. Mass spectral signals for neat drug standards prepared at $200 \mathrm{ng} / \mathrm{mL}$ can be seen in channels 1-4 while analyte signals for spiked meconium standards containing $200 \mathrm{ng} / \mathrm{mL}$ of each analyte can be seen in channels 5-8.

Figures 46-48 clearly illustrate the effect of the complex meconium matrix on the analyte signal. While signal intensities were reasonable for all analytes sampled as dilute drug standards, signals all but diminished when analytes were sampled at the same concentrations in the meconium matrix. The negative effect of the meconium matrix on analyte signal intensity meant that additional sample preparation/cleanup was required to achieve the desired screening sensitivity. Multiple sample preparation techniques were investigated in the hope that mass spectral responses for all analytes could be significantly enhanced without the need for extensive sample preparation. 


\subsubsection{Meconium Sample Preparation Method \#1}

Meconium specimens employed for all DESI-MS/MS experiments were accurately weighed and diluted by a factor of $3(\mathrm{w} / \mathrm{v})$ with $50: 50$ methanol/water to assist with the sonication procedure required for immunoassay screening. In an attempt to clean up the sample and increase sensitivity by eliminating some of the matrix effects, the following sample preparation procedure was applied to blank meconium specimens that had been spiked with appropriate analytes:

1. Combine $1 \mathrm{~mL}$ of the meconium homogenate with $2 \mathrm{~mL}$ of cold acetonitrile

2. Vortex sample for 45 seconds

3. Centrifuge sample for 2 minutes at $3000 \mathrm{rpm}$

4. Transfer $500 \mu \mathrm{L}$ of the organic layer to clean test tube and evaporate to dryness

5. Reconstitute in $50 \mu \mathrm{L}$ of $\mathrm{ACN}$ and spot $2 \mu \mathrm{L}$ directly onto Omni Slide ${ }^{\mathrm{TM}}$ for analysis

Following sample preparation, reconstituted samples were directly spotted onto the surface of the Omni Slide ${ }^{\mathrm{TM}}$. Resulting analyte signals for the amphetamines and cocaine derivatives can be seen in Figures 49 and 50. 


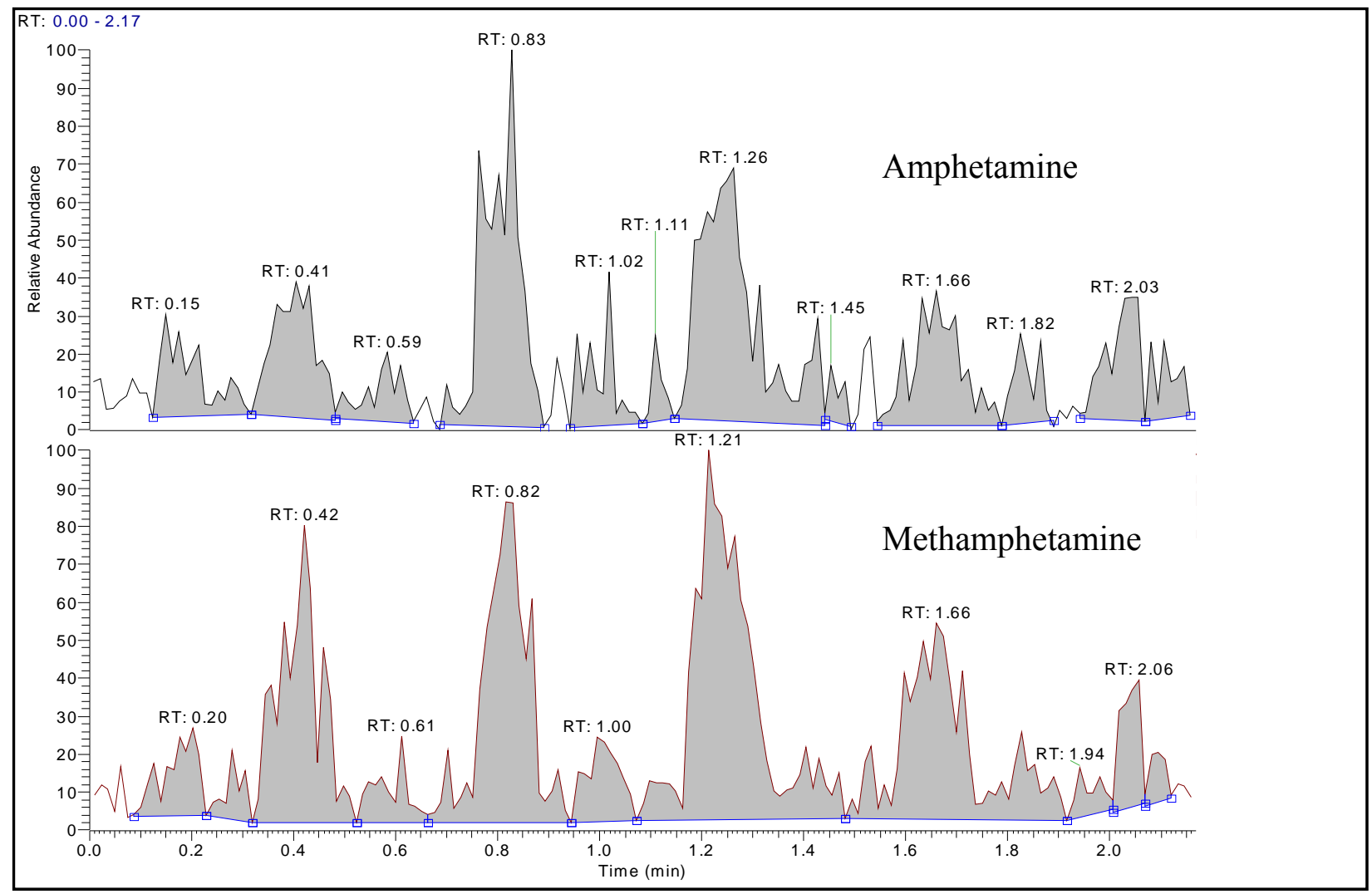

Figure 49: DESI-MS/MS signal for the amphetamines following limited sample preparation according to sample preparation method \#1. 


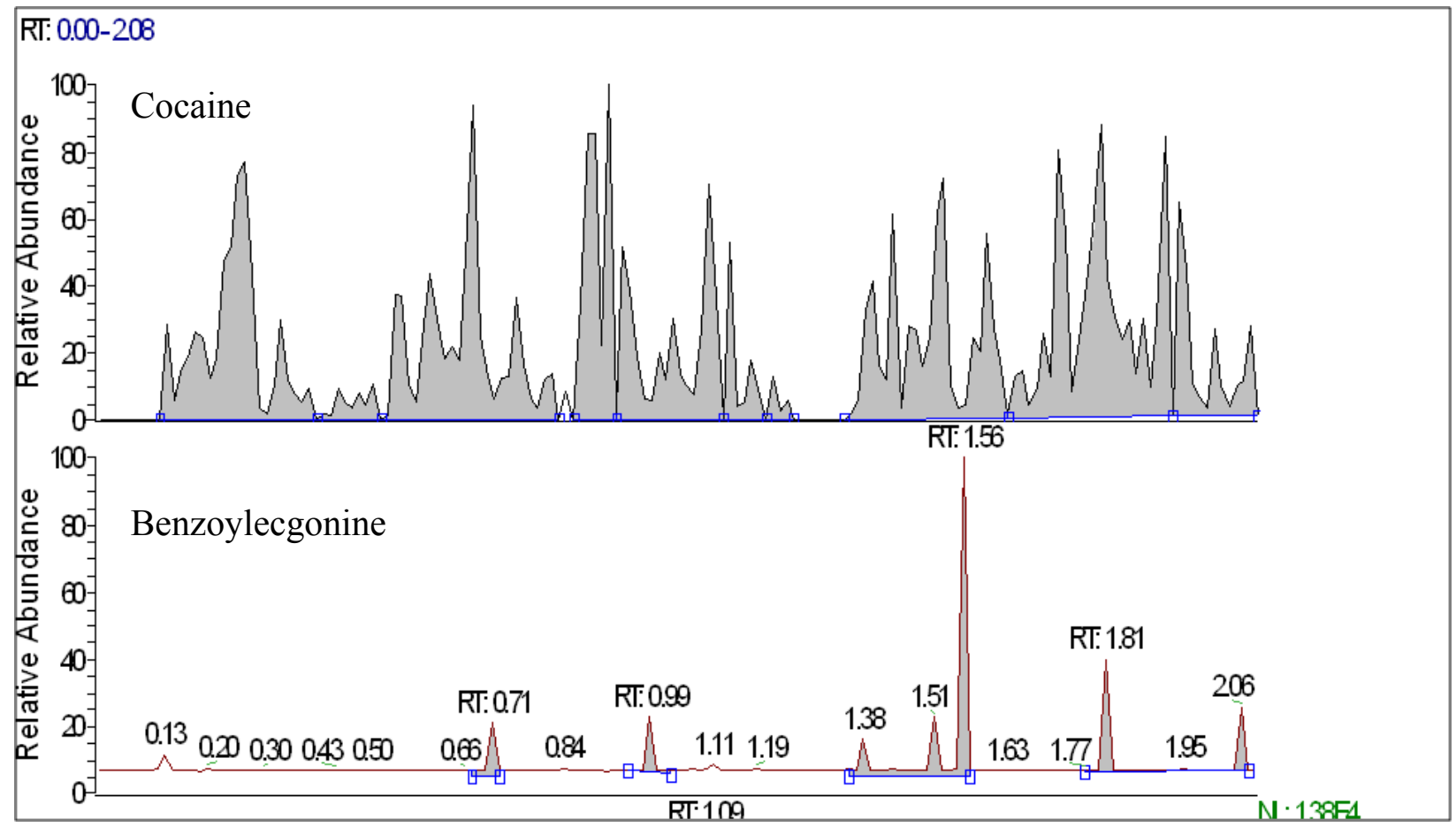

Figure 50: DESI-MS/MS signal for cocaine and benzoylecgonine following limited sample preparation.

Signal intensities for both amphetamine and methamphetamine were only marginally enhanced following the additional sample cleanup (Figure 49). The combination of precipitating out many of the endogenous proteins and concentrating the sample by drying down appears to have eliminated some of the matrix components responsible for signal suppression and slightly increased sensitivity. While the additional sample cleanup appears to slightly increase the mass spectral response for both analytes, the signal to noise $(\mathrm{S} / \mathrm{N})$ ratio is still very low making identification of the sample spots very difficult (Figure 49). The added step of concentrating the analyte has obvious advantages with regard to sensitivity, however, the process of drying down and reconstituting can be time consuming and laborious and must be kept minim completely eliminated, during screening procedures. Introducing such steps into screening procedures greatly reduces the efficiency of the screen by increasing time and labor costs 
associated with sample preparation. Screening techniques should provide accurate, qualitative information without the need for extensive sample preparation.

Although signal intensities for benzoylecgonine remained very poor (Figure 50), the additional sample clean up appears to have significantly enhanced the DESI-MS/MS response for cocaine. While sensitivity has long been recognized as a downfall associated with ambient pressure ionization techniques, preliminary data reveals yet another complicating factor which needs to be addressed when developing and optimizing experimental protocols for DESI. Figures 49 and 50 clearly illustrate the potential for sample redistribution leading to a continuous analyte response over the entire surface of the Omni Slide ${ }^{\mathrm{TM}}$. If sample spots remained confined to the sample wells on the surface of the Omni Slide ${ }^{\mathrm{TM}}$ a distinct number of analyte responses corresponding to the number of spotted samples would be observed. The continuous analyte response seen in Figure 50 indicates that the meconium matrix may be susceptible to redistribution on the surface of the Omni Slide ${ }^{\mathrm{TM}}$ leading to indistinguishable sample spectra.

During initial experiments involving the Omni Slides ${ }^{\mathrm{TM}}$, it was repeatedly noted that samples prepared in the meconium matrix did not adhere very well to the surface of the slide. This observation provides a likely explanation for the sample redistribution seen in Figure 50. When meconium based samples are spotted onto the Omni Slide ${ }^{\mathrm{TM}}$, they redistribute outside the boundaries of the sample channel. In addition to this phenomenon, spray solvents tend to dissolve on the surface on Omni Slides ${ }^{\mathrm{TM}}$ causing further sample redistribution which can lead to cross contamination from one sample channel to the next.

Poor signal intensities were again observed for codeine, morphine, and hydromorphone even following additional sample cleanup. This factor, combined with the significant signal 
enhancements noticed for amphetamine, methamphetamine, and cocaine following identical sample cleanup, suggest that the poor sensitivities associated with several morphine derivatives may be due to limitations of the ionization process itself rather than matrix components causing suppression.

Interestingly, sample redistribution leading to indistinguishable analyte responses did not appear to be in issue during the analysis of drugs standards prepared in methanol (Figure 44). This observation suggests that a more efficient sample preparation capable of removing additional matrix components may reduce the extent of sample redistribution on the hydrophobic surface of the Omni Slide ${ }^{\mathrm{TM}}$ which can lead to contamination in surrounding sample channels. Various techniques for reducing the degree of sample redistribution require investigation, however, significant enhancements in sensitivity need first be achieved before the DESI-MS/MS methodology can be considered useful for any practical screening applications. Meconium sample preparation method \#2 was developed for the purpose of increasing sensitivity and possibly reducing the degree of sample redistribution.

\subsubsection{Meconium Sample Preparation Method \#2}

Preliminary experiments indicate that the removal of complex matrix components causing signal suppression will not only increase sensitivity for all analytes but may also decrease sample redistribution on the hydrophobic surface of the Omni Slide ${ }^{\mathrm{TM}}$ which can lead to sample redistribution and indistinguishable analyte responses. In order to evaluate the use of an additional filtration step for sample cleanup, sample preparation method $\# 2$ was investigated. In addition to the protein crash employing cold acetonitrile it was hoped that by filtering the organic phase through $0.1 \mu \mathrm{m}$ Durapore PVDF filter units, additional sample cleanup would be achieved 
leading to greater signal intensities and less sample redistribution. Samples were prepared as follows:

1. Combine $1 \mathrm{~mL}$ of the meconium homogenate with $2 \mathrm{~mL}$ of cold acetonitrile

2. Vortex sample for 45 seconds

3. Centrifuge sample for 2 minutes at $3000 \mathrm{rpm}$

4. Filter organic layer through Durapore PVDF $0.1 \mu \mathrm{m}$ filter units

5. Spot $2 \mu \mathrm{L}$ directly onto Omni Slide ${ }^{\mathrm{TM}}$ for analysis

Four sample spots of meconium matrix prepared according to meconium sample preparation method \#2 were analyzed directly adjacent to neat drug standards (positive control) prepared at appropriate concentrations. Positive quality control (QC) specimens had been prepared by diluting neat drug standards $(1 \mathrm{mg} / \mathrm{mL}$ in methanol) to appropriate screening concentrations. Experiment were designed to evaluate the usefulness of analyzing positive controls prior to scanning authentic patient samples for the purpose of identifying true positive samples through direct comparison of analyte responses. Samples were spotted onto the Omni Slides ${ }^{\mathrm{TM}}$ according to Figure 51.

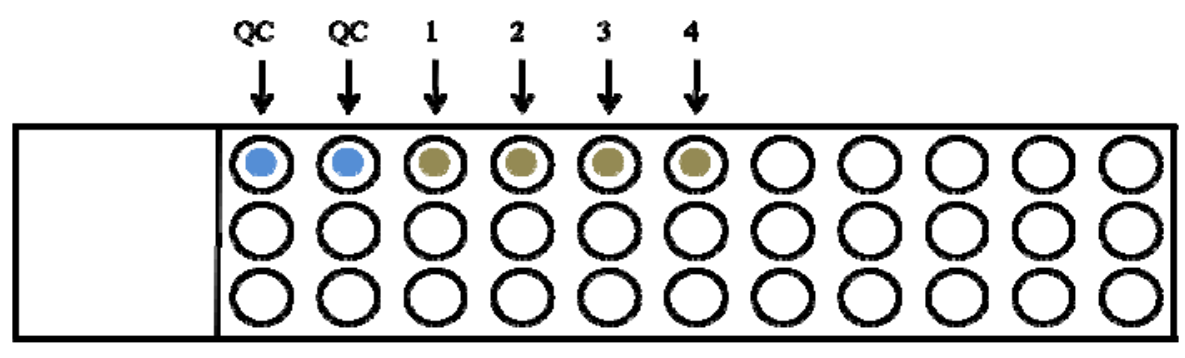

Figure 51: Previously prepared positive quality control (QC) samples were spotted in the first two sample channels to assist with identifying positive authentic samples spotted in channels 3-6. 
Figure 52 illustrates the analyte responses for a cocaine/benzoylecgonine quality control standard prepared at $50 \mathrm{ng} / \mathrm{mL}$ spotted in channels 1-2 and analyte responses for a blank meconium specimen spiked with cocaine and benzoylecgonine at a concentration of $250 \mathrm{ng} / \mathrm{mL}$ and spotted in sample channels 3-6. All positive quality control standards were prepared from neat drug standards ( $1 \mathrm{mg} / \mathrm{mL}$ in methanol) through serial dilutions.

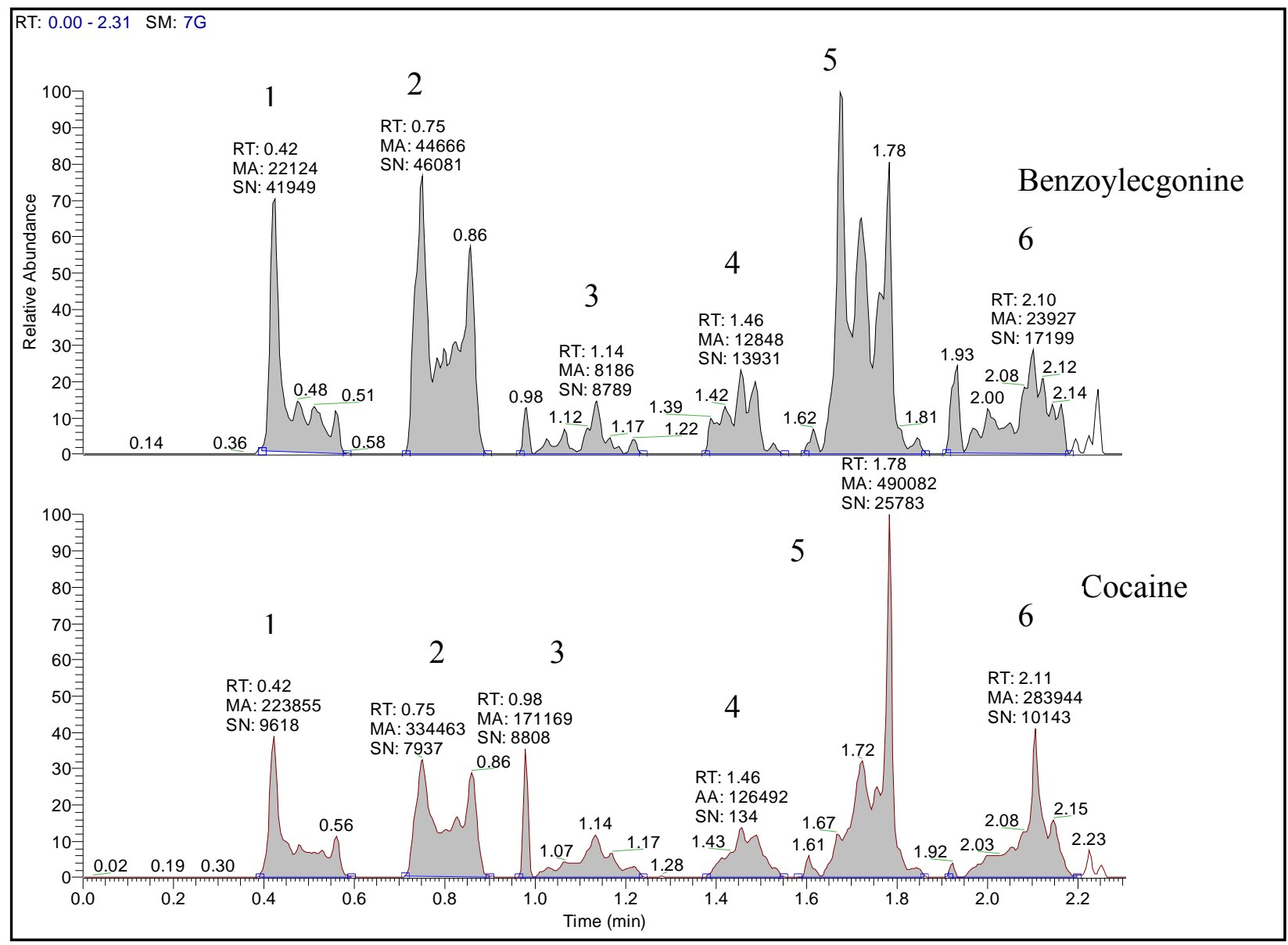

Figure 52: Sample channels 1-2 represent analyte responses for a cocaine and benzoylecgonine quality control (QC) standard $(50 \mathrm{ng} / \mathrm{mL})$ while sample channels 3-6 were spotted with spiked meconium samples $(250 \mathrm{ng} / \mathrm{mL})$.

Cocaine and benzoylecgonine signals appear to have been significantly enhanced using the additional filtration step described in sample preparation method \#2. The filtration removal of 
endogenous compounds in the meconium matrix has greatly enhanced the benzoylecgonine signal which was undetectable using sample preparation method \#1. While the non-linear response between the quality control standard $(50 \mathrm{ng} / \mathrm{mL})$ and the spiked meconium $(250 \mathrm{ng} / \mathrm{mL})$ indicates that there is still some degree of signal suppression, the additional filtration step appears to have reduced this effect and enhanced overall sensitivity. The additional preparation step has also appears to have reduced the degree of sample redistribution. This is evident by the distinct analyte responses corresponding to individual sample spots on the surface of the Omni Slide ${ }^{\mathrm{TM}}$.

Figure 53 shows the mass spectral response of a cocaine/benzoylecgonine positive control prepared at $50 \mathrm{ng} / \mathrm{mL}$ and spotted in channels 1-3 and analyte responses for a blank meconium specimen spotted in channels 4-6. 


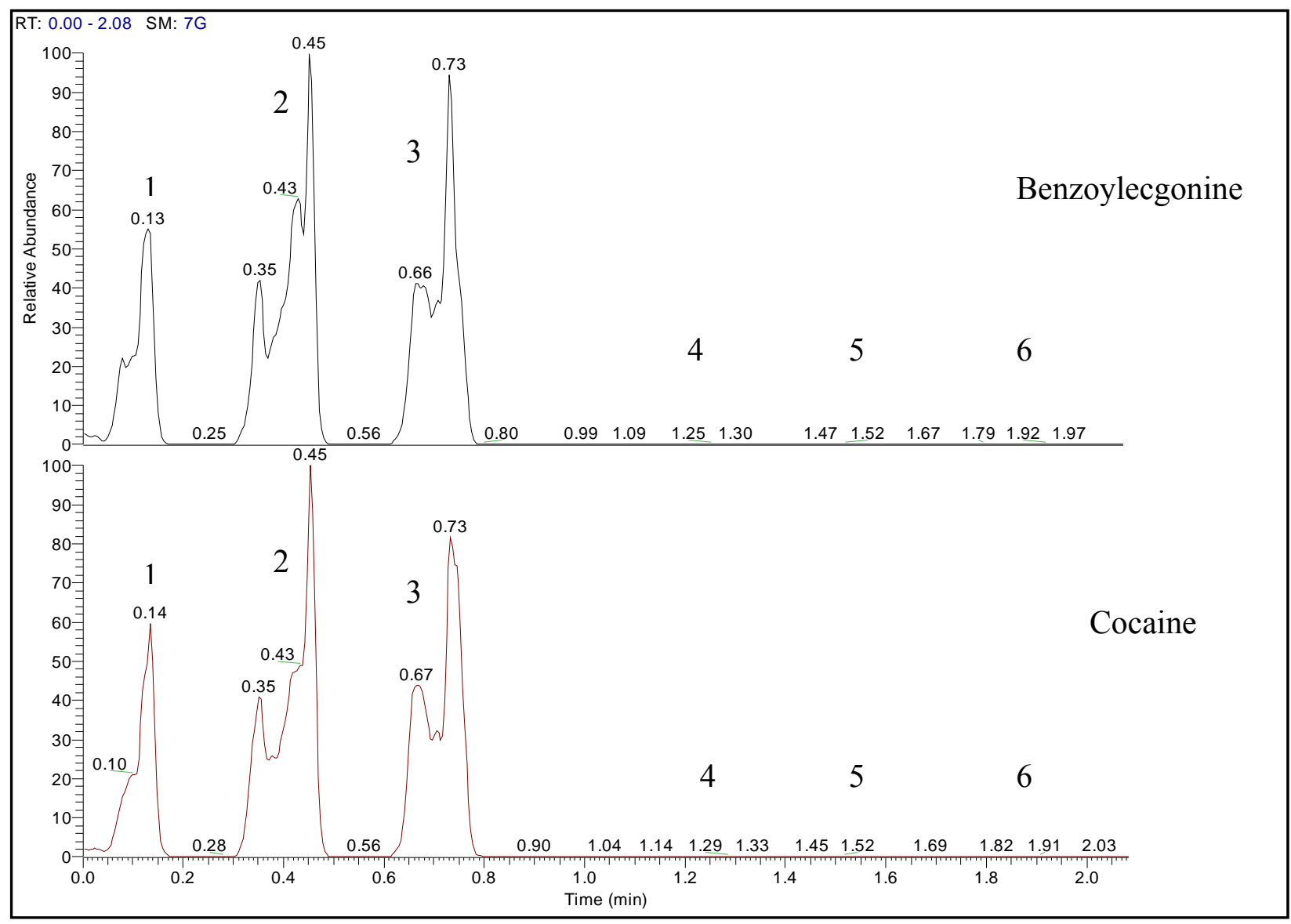

Figure 53: Mass spectral responses for a cocaine/benzoylecgonine positive control $(50 \mathrm{ng} / \mathrm{mL})$ in channels 1-3 and analyte responses for a blank meconium specimen spotted in channels 4-6.

Blank meconium specimens spotted in channels 4-6 were negative for cocaine and benzoylecgonine when scanned using the 2 MRM transition MS/MS method.

Figure 54 shows the mass spectral response of an amphetamine/methamphetamine positive control sample prepared at a concentration of $50 \mathrm{ng} / \mathrm{mL}$ and spotted in channels $1-2$ and analyte responses for a meconium specimen spiked with amphetamine and methamphetamine at a concentration of $500 \mathrm{ng} / \mathrm{mL}$ and spotted in channels 3-6. 


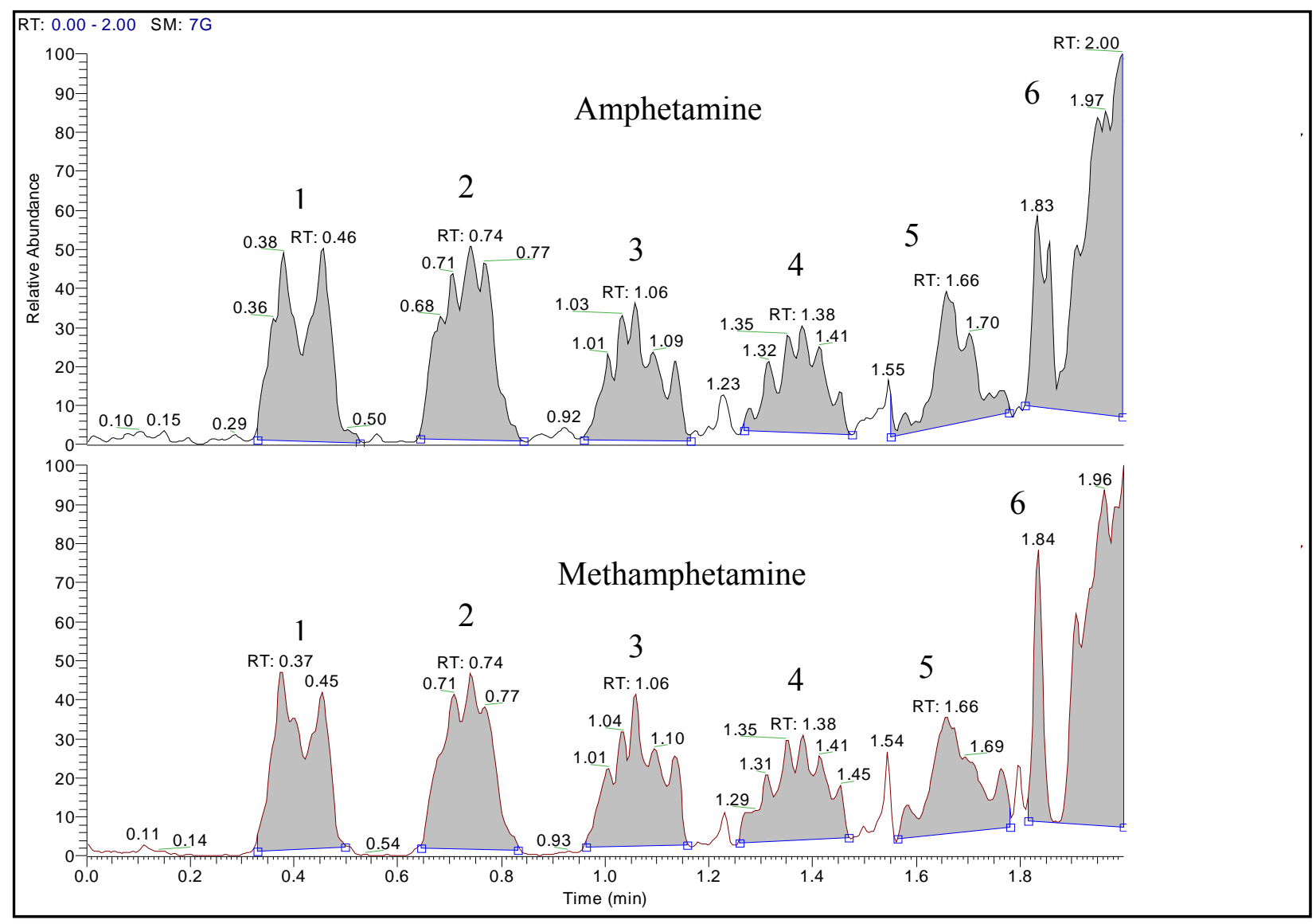

Figure 54: Mass spectral response of an amphetamine/methamphetamine positive control sample (50 ng/mL) spotted in channels 1-2 and analyte responses for a meconium specimen spiked with amphetamine and methamphetamine $(500 \mathrm{ng} / \mathrm{mL})$ in channels 3-6.

Meconium sample preparation method \#2 also enhanced the mass spectral response for amphetamine and methamphetamine in the meconium matrix (Figure 54). While both analytes were detected in the meconium matrix using the initial sample preparation method, sensitivities were poor and appear to have been greatly enhanced with the additional filtration step. The S/N ratio has been increased using sample preparation method $\# 2$ and the amphetamine signals are now more clearly distinguished from background noise. Distinct analyte signals for amphetamine and methamphetamine are also seen in Figure 54 indicating that sample redistribution has been minimized/eliminated using the new preparation method. Although analyte signals are 
significantly enhanced following the additional filtration step, direct comparison of the signals from the $50 \mathrm{ng} / \mathrm{mL}$ quality control and the $500 \mathrm{ng} / \mathrm{mL}$ spiked sample indicate that matrix effects still exist.

Figure 55 shows the mass spectral signals for an amphetamine/methamphetamine positive control $(50 \mathrm{ng} / \mathrm{mL})$ spotted in sample channels 1-2 and analyte responses for a blank meconium specimen prepared according to sample preparation method $\# 2$ and spotted in channels 3-6.

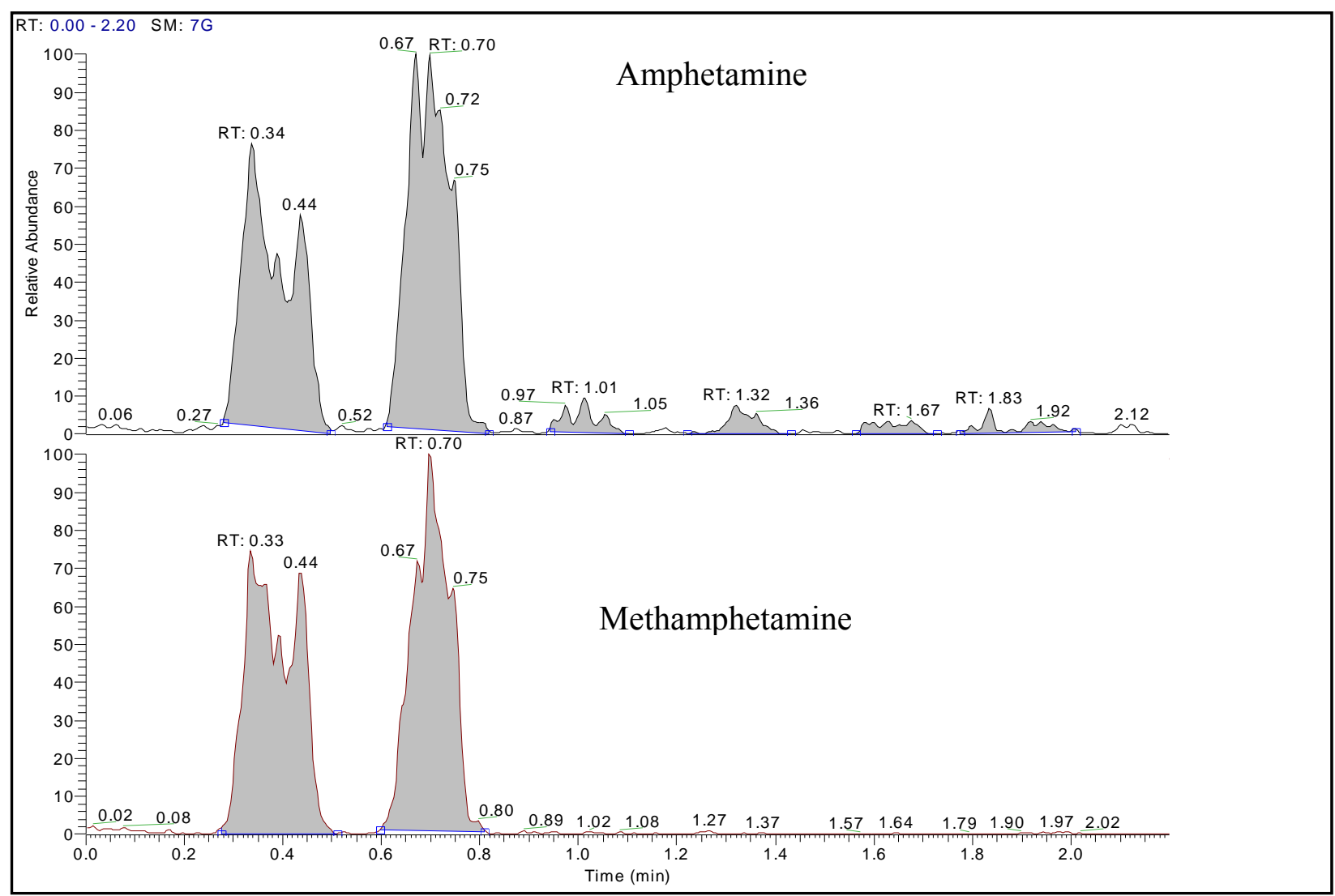

Figure 55: Mass spectral response of an amphetamine/methamphetamine positive control sample $(50 \mathrm{ng} / \mathrm{mL})$ spotted in channels $1-2$ and analyte responses for a blank meconium specimen spotted in channels 3-6. 
Blank meconium specimens spotted in sample channels 3-6 did not generate any appreciable analyte response for either amphetamine or methamphetamine (Figure 55).

Figure 56 shows the mass spectral response for an opiate positive control specimen (500 $\mathrm{ng} / \mathrm{mL}$ ) spotted in sample channels 1-2 and analyte responses for a blank meconium specimen spiked with morphine, codeine, and hydromorphone at a concentration of $500 \mathrm{ng} / \mathrm{mL}$.

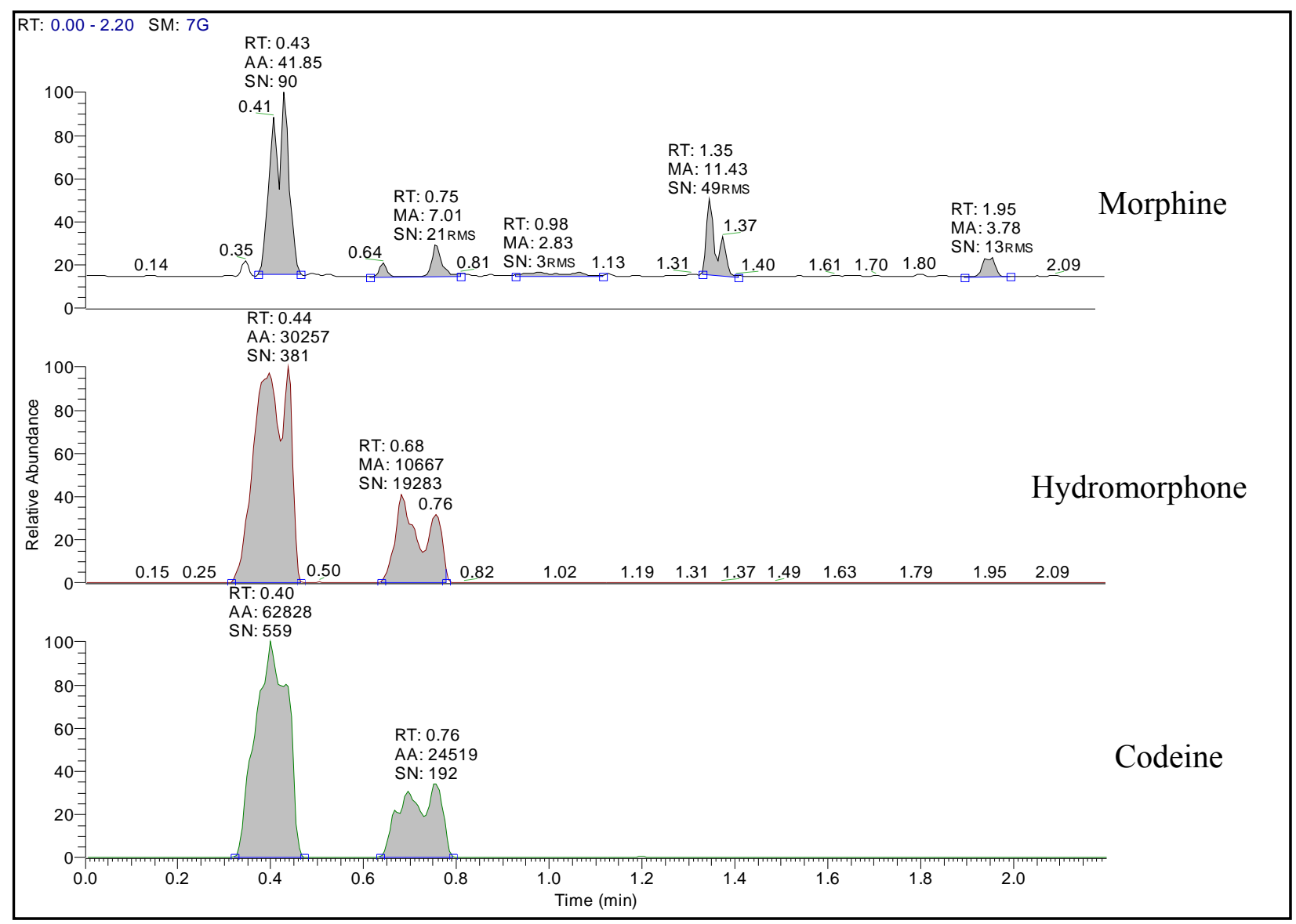

Figure 56: Mass spectral response of an opiate positive control sample $(500 \mathrm{ng} / \mathrm{mL})$ spotted in channels 1-2 and analyte responses for a meconium specimen spiked with opiates $(500 \mathrm{ng} / \mathrm{mL})$ and spotted in channels 3-6.

Extremely poor analyte signals were again observed for all opiate analytes in both the spiked meconium specimen and the positive quality control specimen. Poor analyte signals observed for 
the $500 \mathrm{ng} / \mathrm{mL}$ neat drug standard supported earlier hypotheses that chemical properties of the opiates combined with DESI mechanisms may be responsible for the low analyte signals as opposed to matrix effects. While evidence of matrix suppression does exist, the inferior sensitivity of the opiates relative to the amphetamine and cocaine derivatives suggests that the morphine derivatives may not readily lend themselves to DESI, leading to inefficient ion formation. Efficient extraction and desorption of the analyte from the surface of interest is vital for any DESI experiment. Without efficient extraction and desorption from the surface, analyte ionization will be limited leading to decreased sensitivities. Studies indicate that efficient recovery of opiate derivatives from the meconium matrix may not be feasible using DESI.

Although signal intensities were consistently poor for all opiate derivatives, significant signal enhancement for the amphetamine and cocaine derivatives indicated that the sample filtration step reduced signal suppression and increased overall sensitivity. In order to evaluate the increased sensitivity afforded by filtering the organic phase, meconium specimens were prepared with and without the filtration step and analyzed side by side. Positive quality control specimens prepared through serial dilutions of neat drug standards were again analyzed prior to authentic specimens to assist with positive identifications. All positive quality control specimens were spotted in sample channel 2 while channels 1 and 3 were left empty. Meconium samples prepared using sample preparation method \#1 were spotted in sample channels 4-6 and meconium samples prepared using the additional filtration step described in sample preparation method \#2 were spotted in sample channels 7-9. Figure 57 shows the mass spectral responses of amphetamine and methamphetamine standards (a-b) as well as benzoylecgonine and cocaine standards (c-d) which were spotted in channel 2. Channels 4-6 were spotted with meconium which had been spiked with amphetamine and methamphetamine and prepared according to 
sample preparation method \#1. Channels 7-9 were spotted with meconium which had been spiked with amphetamine and methamphetamine and prepared according to sample preparation method \#2. The MS/MS method consisted of four MRM transitions, one for each of the analytes. Side by side analyses were performed to determine the extent to which the additional filtration step increases overall sensitivity.

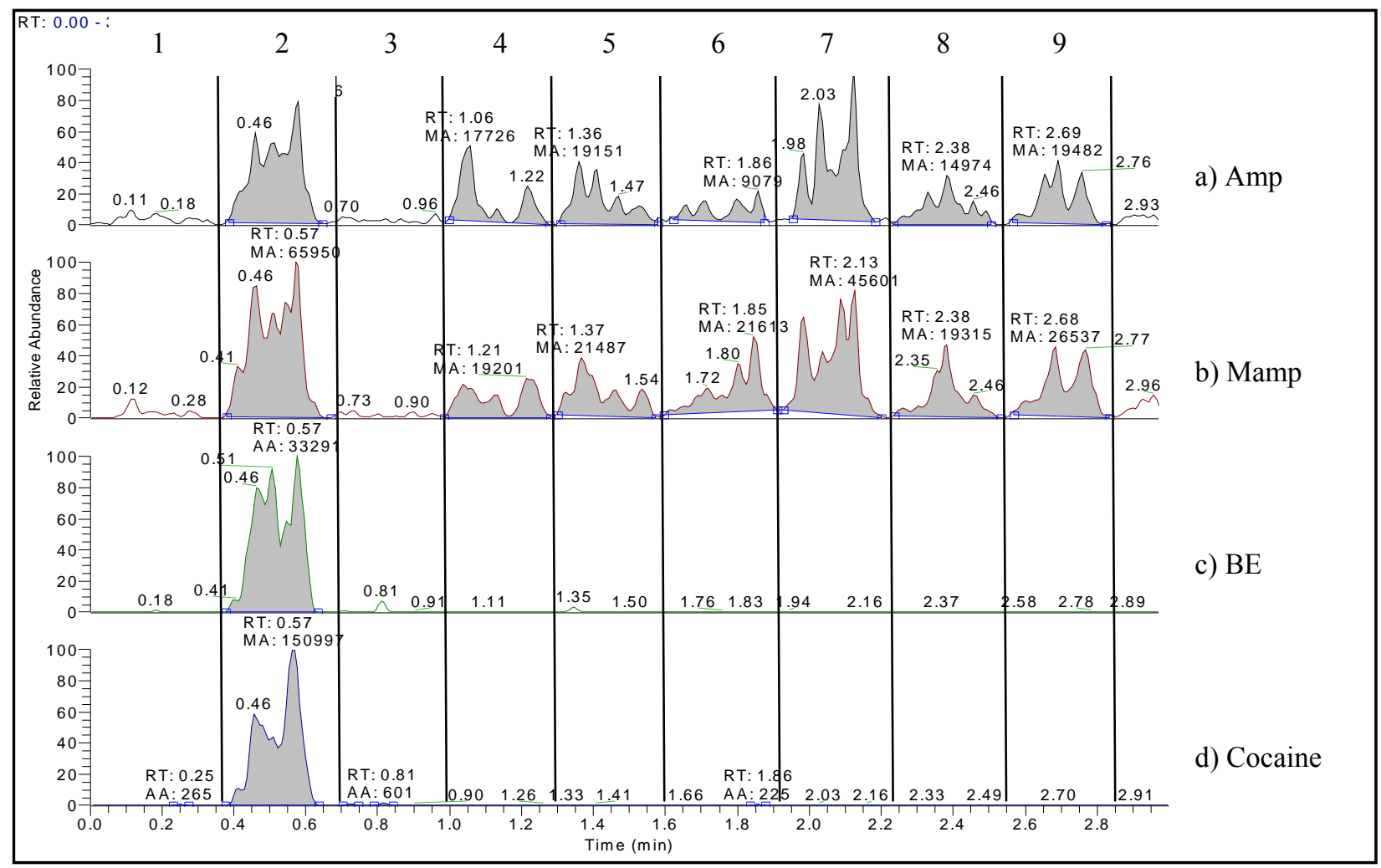

Figure 57: Mass spectral responses of amphetamine and methamphetamine standards (a-b) as well as benzoylecgonine and cocaine standards (c-d) spotted in channel 2. Mass spectral responses in channels 4-6 represent meconium samples which had been spiked with amphetamine and methamphetamine $(500 \mathrm{ng} / \mathrm{mL})$ and prepared according to sample preparation method \#1. Mass spectral responses in channels 7-9 represent meconium specimens which had been spiked with amphetamine and methamphetamine $(500 \mathrm{ng} / \mathrm{mL})$ and prepared according to sample preparation method \#2. 
Both the amphetamine and cocaine positive quality control specimens were prepared at a concentration of $25 \mathrm{ng} / \mathrm{mL}$. Rows a-b in Figure 57 show the mass spectral responses for the positive quality control specimen (sample channel 2) and a meconium specimen spiked with amphetamine and methamphetamine at a concentration of $500 \mathrm{ng} / \mathrm{mL}$ (channels 4-9). Samples spotted in channels 7-9 had undergone additional filtration to remove matrix components responsible for signal suppression. Only a small increase in signal intensity is seen for the filtered samples indicating that the time expended performing the filtration step may not provide significant increases in sensitivity to warrant its inclusion in the method.

In an attempt to further enhance sensitivity, the mass spectrometry method was separated into two separate methods each containing 2 MRM transitions. By separating the mass spectral method into two components, each consisting of 2 MRM transitions, it was hoped that dwell time, and therefore sensitivity, could be maximized. The MS/MS method was divided into two separate methods, one containing the MRM transitions for amphetamine and methamphetamine, the other containing MRM transitions for cocaine and benzoylecgonine. Meconium specimens which had been spiked with amphetamine and methamphetamine were first analyzed using the combined MS/MS method (Figure 57) and then using the specific amphetamine/methamphetamine method (Figure 58). Specimens which had been spiked with cocaine and benzoylecgonine were first analyzed using the combined MS/MS method (Figure 59) and then using the specific cocaine/BE method (Figure 60). It was hoped that by reducing the number of MRM transitions within each mass spectral method, improvements in sensitivity would be observed. Any gains in sensitivity would obviously come at the cost of analysis time, as samples would require analysis by two specific mass spectral methods as opposed to one combined method containing all four MRM transitions. 


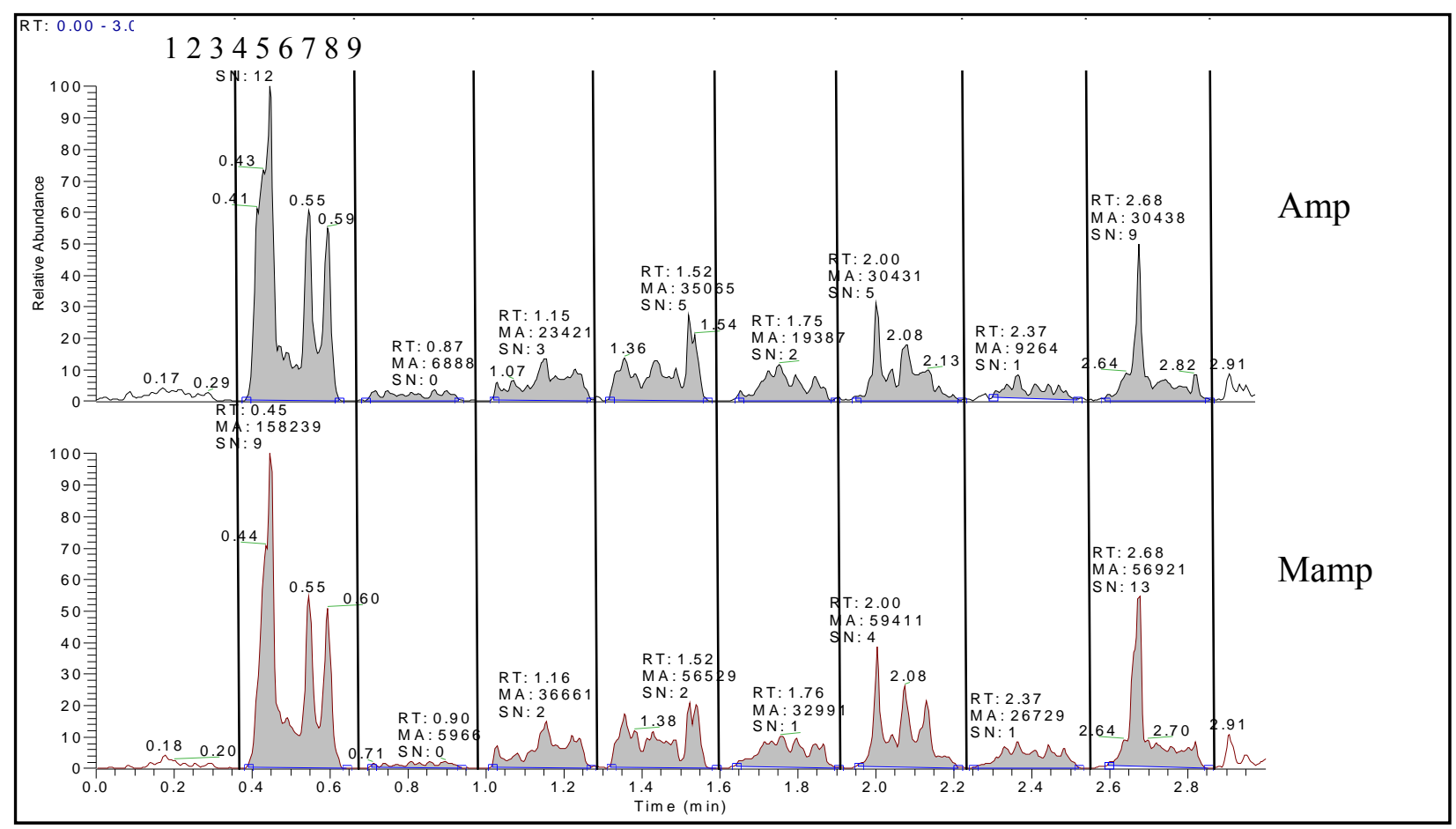

Figure 58: Mass spectral responses for amphetamine and methamphetamine with the amphetamine/methamphetamine specific MS/MS method containing 2 MRM transitions. Sample channel 2 contains a positive quality control specimen prepared at $25 \mathrm{ng} / \mathrm{mL}$. Sample channels 46 contain meconium matrix spiked with amphetamine and methamphetamine $(500 \mathrm{ng} / \mathrm{mL})$ and prepared according to sample preparation method \#1. Sample channels 7-9 contain meconium matrix spiked with amphetamine and methamphetamine $(500 \mathrm{ng} / \mathrm{mL})$ and prepared according to sample preparation method \#2.

Figure 59 shows the mass spectral responses for amphetamine and methamphetamine in both the positive quality control specimen and the spiked meconium matrix. Mass spectral responses were obtained using the amphetamine specific MS/MS method containing one MRM transition for each analyte. Small improvements in signal intensities were observed for amphetamine and methamphetamine when scanning with the $2 \mathrm{MRM}$ transition method. 


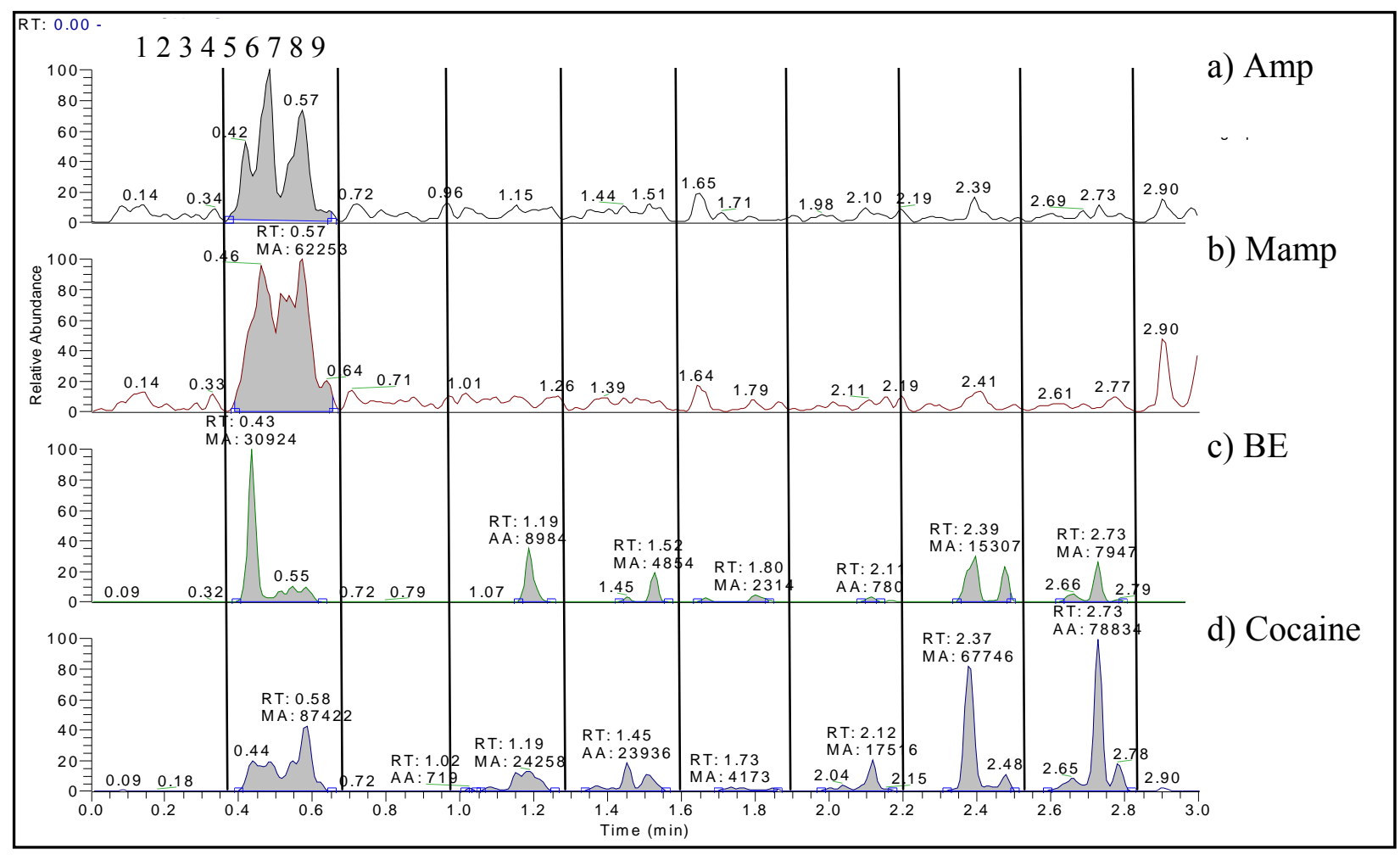

Figure 59: Mass spectral responses of amphetamine and methamphetamine standards (a-b) as well as benzoylecgonine and cocaine standards (c-d) spotted in channel 2. Mass spectral responses in channels 4-6 represent meconium samples which had been spiked with cocaine and benzoylecgonine $(250 \mathrm{ng} / \mathrm{mL})$ and prepared according to sample preparation method \#1. Mass spectral responses in channels 7-9 represent meconium specimens which had been spiked with cocaine and benzoylecgonine $(250 \mathrm{ng} / \mathrm{mL})$ and prepared according to sample preparation method \#2.

While mass spectral responses for cocaine and benzoylecgonine in the meconium matrix (sample channels 4-9) were relatively low in intensity, significant signal enhancement was observed for specimens which had been filtered through Durapore PVDF $0.1 \mu \mathrm{m}$ filter units (sample channels 7-9). Figure 59 indicates that filtration of the acetonitrile layer following protein precipitation may aid in the removal of endogenous compounds responsible for the suppression of cocaine and benzoylecgonine signals. Filtration of the organic layer did not appear to enhance the amphetamine/methamphetamine signal to the same degree, however, small 
increases in the cocaine/BE signals were more easily observed due to the complete absence of signal in unfiltered samples.

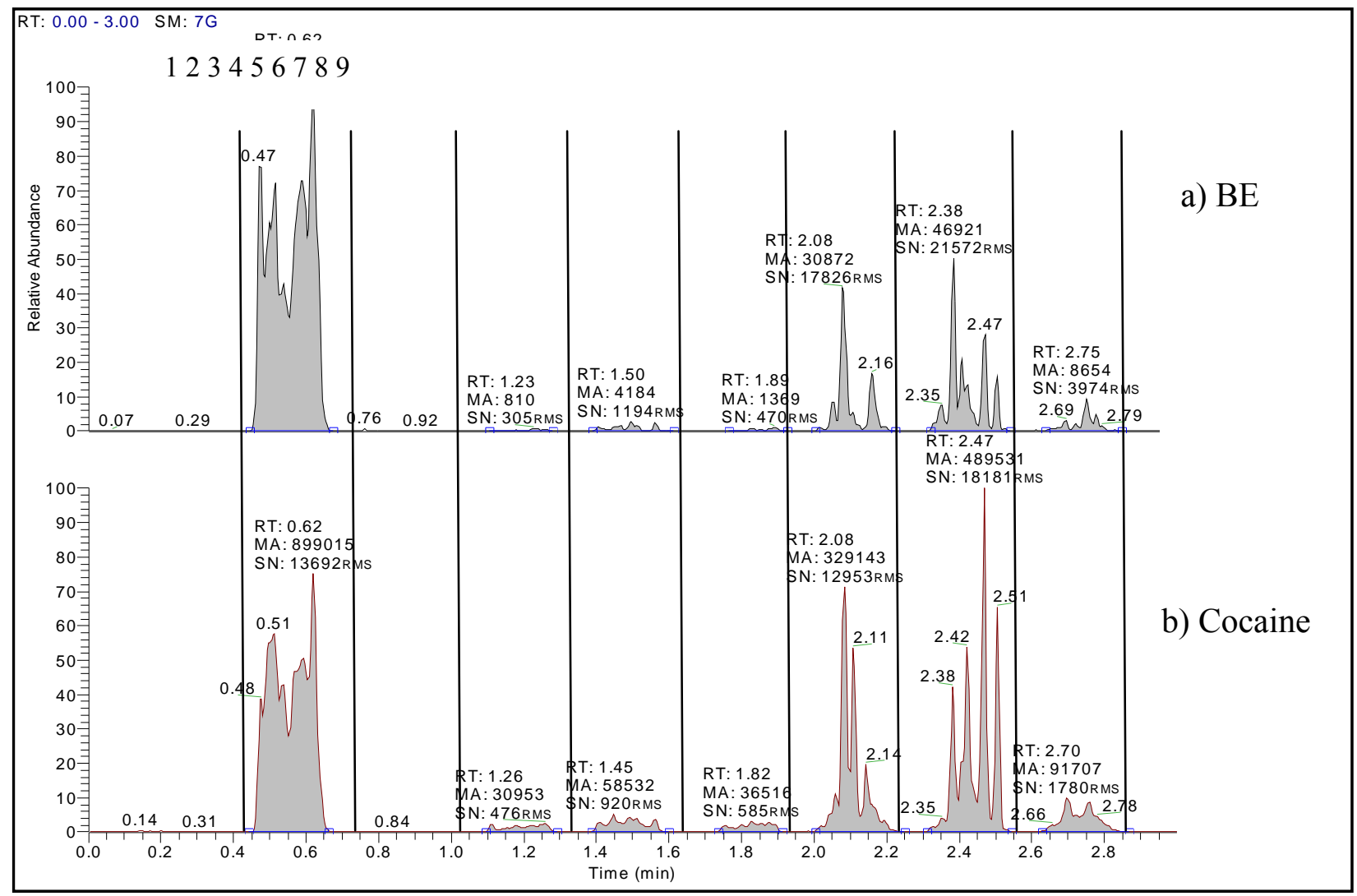

Figure 60: Mass spectral responses of benzoylecgonine and cocaine standards (a-b) spotted in channel 2. Mass spectral responses in channels 4-6 represent unfiltered meconium samples which had been spiked with cocaine and benzoylecgonine and analyzed using the cocaine/BE specific MS/MS method consisting of one MRM transition for each analyte. Mass spectral responses in sample channels 7-9 represent filtered meconium specimens analyzed by the same method.

The increased mass spectral responses observed in Figure 60 for cocaine and benzoylecgonine indicate that better sensitivities may be afforded by the 2 MRM transition method. Further studies into the extent of this signal enhancement are required to evaluate the suitability of employing multiple mass spectral methods when scanning authentic samples. While reducing the number of transitions in a mass spectral method allows for longer dwell times and 
greater sensitivity, separating a comprehensive MS/MS method into multiple methods containing fewer MRM transitions can be both time and cost ineffective. If a specimen is to be screened for the presence of amphetamines and cocaine, analysis time will be minimized using a mass spectral method that simultaneously screens for both drug classes. Separating this comprehensive method into two separate MS/MS methods would allow for slightly greater sensitivities, but would also mean that samples would need to be analyzed twice, once for each method. This would have obvious drawbacks in high throughput toxicology laboratories as analysis time would be essentially doubled. The advantage of scanning each sample spot twice would be the increase in sensitivity afforded by the MS/MS method comprising fewer MRM transitions.

Detailed experiments aimed at evaluating the disadvantages of increased analysis time versus the advantages of increases sensitivities must be performed before conclusions can be drawn. The analysis of dilute drug standards prior to authentic patient samples appears to significantly aid in the identification of true positives. Due to the complex nature of the meconium specimens, low level background signal is often observed when analyzing blank specimens. Differentiating between background signal attributable to endogenous matrix components and true positive samples can be difficult without the use of a positive quality control specimen to act as a reference. By analyzing positive quality control specimens prepared at appropriate concentrations prior to authentic patient samples, it is hoped that low level background signal will be easily distinguished from authentic analyte responses. 


\subsection{Qualitative Determination of Amphetamine, Methamphetamine, Cocaine, and Benzoylecgonine in Authentic Meconium Specimens using DESI-MS/MS.}

In order to evaluate the usefulness of DESI-MS/MS for screening authentic meconium specimens for drugs of abuse, direct comparative studies were performed with existing immunoassay techniques. While preliminary experiments involving spiked meconium specimens are satisfactory for 'proof of concept' studies, no insight into the overall applicability of the method can be gained until it is applied to authentic samples.

\subsubsection{Experimental Design}

Authentic meconium specimens $(n=21)$ which had previously screened positive at AIT Laboratories (Indianapolis, IN) for amphetamine and/or cocaine were selected for the studies aimed at directly comparing the screening accuracy of DESI-MS/MS to the pre-existing immunoassay technique. Immunoassay screening results are shown in Table 57. All specimens chosen for this study were leftover from routine analysis performed at AIT Laboratories. Samples were selected based solely on initial screening results and were given arbitrary identification numbers to ensure that all samples were blinded and could not be connected to individuals at any point during the investigation. 
Table 57: Immunoassay screening results for experimental specimens.

\begin{tabular}{|c|c|c|}
\hline Specimen \# & Amphetamine screen (ELISA) & Cocaine/BE screen (ELISA) \\
\hline 1 & Positive & Negative \\
\hline 2 & Positive & Negative \\
\hline 3 & Negative & Positive \\
\hline 4 & Negative & Positive \\
\hline 5 & Positive & Negative \\
\hline 6 & Negative & Positive \\
\hline 7 & Positive & Negative \\
\hline 8 & Negative & Positive \\
\hline 9 & Negative & Positive \\
\hline 10 & Negative & Positive \\
\hline 11 & Negative & Positive \\
\hline 12 & Positive & Negative \\
\hline 13 & Negative & Positive \\
\hline 14 & Positive & $\overline{\text { Negative }}$ \\
\hline 15 & Negative & Positive \\
\hline 16 & Positive & Negative \\
\hline 17 & Negative & Positive \\
\hline 18 & Positive & Negative \\
\hline 19 & Positive & Positive \\
\hline 20 & Positive & Positive \\
\hline 21 & Negative & Positive \\
\hline
\end{tabular}

Ten specimens screened positive for amphetamines using the immunoassay screening technique while thirteen of the meconium specimens screened positive for cocaine/benzoylecgonine (Table 57). All specimens $(n=21)$ were then screened using the DESIMS/MS methodology. 


\subsubsection{Sample Preparation for DESI-MS/MS Screening Experiments}

Sample preparation for DESI-MS/MS screening was as follows:

1. Combine $1 \mathrm{~mL}$ of the meconium homogenate with $2 \mathrm{~mL}$ of cold acetonitrile

2. Vortex sample for 45 seconds

3. Centrifuge sample for 2 minutes at $3000 \mathrm{rpm}$

4. Filter organic layer through Durapore PVDF $0.1 \mu \mathrm{m}$ filter units

5. Transfer and spot $1.5 \mu \mathrm{L}$ of the acetonitrile phase directly onto the Omni Slide ${ }^{\mathrm{TM}}$

By eliminating the dry down and reconstitution steps, sample preparation was significantly

reduced. Following sample preparation, samples were spotted onto the Omni Slides ${ }^{\mathrm{TM}}$ according to the Figure 61.

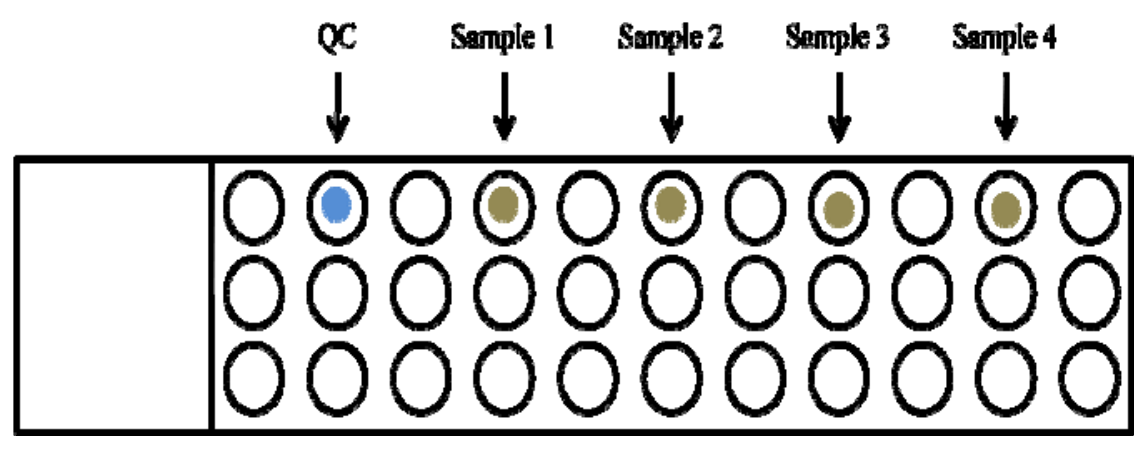

Figure 61: Positive quality control specimens were spotted in channel 2 and analyzed first to aid with positive identifications. Authentic meconium specimens were then spotted in every second sample channel to reduce the possibility of sample redistribution.

\subsubsection{Preparation of Positive Quality Control Specimens}

Positive quality control specimens were again employed for the purpose of aiding in positive identifications. In order to more accurately compare analyte signals from positive quality control specimens to authentic patient specimens, all positive controls were prepared in blank meconium and subjected to the same sample preparation as authentic specimens. 
Preparation of the amphetamine positive control involved spiking the blank meconium matrix with amphetamine and methamphetamine at concentrations of $25 \mathrm{ng} / \mathrm{mL}$. Similarly, the cocaine positive control was prepared by spiking the blank meconium matrix with cocaine and benzoylecgonine at a concentration of $25 \mathrm{ng} / \mathrm{mL}$. By subjecting the positive controls to the same sample preparation as authentic specimens, resulting analyte signals of positive controls will more accurately reflect drug concentrations in patient samples. While unextracted drug standards used in previous experiments were suitable for differentiating an analyte response from background noise, extracted positive QC standards prepared in the meconium matrix will provide more accurate indications as to the presence or absence of a drug in the matrix.

\subsubsection{DESI-MS/MS Parameters}

All DESI source and mass spectrometric parameters were identical to those described in sections 8.3.1 - 8.3.3. Optimized MS/MS parameters for amphetamine, methamphetamine, cocaine, and benzoylecgonine can be found in Table 55. Analysis time was approximately 15 seconds per sample.

\subsubsection{DESI-MS/MS Screening Results}

DESI-MS/MS screening results for all specimens are shown in Table 58. 
Table 58: DESI-MS/MS screening results for authentic meconium specimens $(n=21)$ screened for amphetamines and cocaine.

\begin{tabular}{|c|c|c|}
\hline Specimen \# & Amphetamine screen (DESI) & Cocaine screen (DESI) \\
\hline 1 & Positive & Negative \\
\hline 2 & Negative & Negative \\
\hline 3 & Negative & Negative \\
\hline 4 & Negative & Positive \\
\hline 5 & Positive & Negative \\
\hline 6 & Negative & Positive \\
\hline 7 & Negative & Negative \\
\hline 8 & Negative & Negative \\
\hline 9 & Negative & Negative \\
\hline 10 & Negative & Negative \\
\hline 11 & Negative & Positive \\
\hline 12 & Negative & Negative \\
\hline 13 & Negative & Negative \\
\hline 14 & Positive & Negative \\
\hline 15 & Negative & Negative \\
\hline 16 & Negative & Negative \\
\hline 17 & Negative & Negative \\
\hline 18 & Negative & Negative \\
\hline 19 & Negative & Negative \\
\hline 20 & Negative & Negative \\
\hline 21 & Negative & Positive \\
\hline
\end{tabular}

Fewer positive results were observed with the DESI-MS/MS screening method. Three specimens screened positive for amphetamines while only four specimens screened positive for cocaine/benzoylecgonine (Table 58). Specimens which screened positive using the DESI-

MS/MS method also screened positive on the immunoassay. In order to determine the accuracy of each screening method, all specimens were subject to confirmation analysis using UPLCMS/MS methods described in chapters $5 \& 7$. Confirming the presence or absence of each analyte using UPLC-MS/MS allowed for a more critical evaluation of each screening method with respect to accuracy, selectivity, and sensitivity. Tables 59 and 60 contrast the screening 
results the immunoassay based screen and the DESI-MS/MS screen with confirmed results, respectively.

Table 59: Immunoassay (ELISA) screening results for 21 authentic meconium specimens.

\begin{tabular}{|c|c|c|c|c|}
\hline & $\begin{array}{c}\text { Positive screen } \\
\text { (ELISA) }\end{array}$ & $\begin{array}{c}\text { Positive } \\
\text { confirmation }\end{array}$ & $\begin{array}{c}\text { Rate of false } \\
\text { positives }\end{array}$ & $\begin{array}{c}\text { Rate of false } \\
\text { negatives }\end{array}$ \\
\hline Amphetamines & 10 & 1 & $90 \%$ & $0 \%$ \\
\hline Cocaine & 13 & 4 & $69 \%$ & $0 \%$ \\
\hline
\end{tabular}

Table 60: DESI-MS/MS screening results for 21 authentic meconium specimens.

\begin{tabular}{|c|c|c|c|c|}
\hline & $\begin{array}{c}\text { Positive screen } \\
\text { (DESI) }\end{array}$ & $\begin{array}{c}\text { Positive } \\
\text { confirmation }\end{array}$ & $\begin{array}{c}\text { Rate of false } \\
\text { positives }\end{array}$ & $\begin{array}{c}\text { Rate of false } \\
\text { negatives }\end{array}$ \\
\hline Amphetamines & 3 & 1 & $67 \%$ & $0 \%$ \\
\hline Cocaine & 4 & 4 & $0 \%$ & $0 \%$ \\
\hline
\end{tabular}

All specimens were first screened for amphetamines and cocaine using immunoassay and DESI-MS/MS and then subject to confirmation analysis using UPLC-MS/MS to validate the reliability of each screening technique. Of the 21 meconium specimens making up the experimental sample set, only 5 were confirmed positive for either amphetamines or cocaine. Four specimens were confirmed positive for cocaine and/or benzoylecgonine while only one specimen was confirmed positive for amphetamine (Tables 59 and 60). The 5 positive samples screened positive using both the immunoassay technique and the DESI-MS/MS technique, indicating that neither method is susceptible to generating false negatives. While both methods successfully identified all true positives, the DESI-MS/MS method appeared to offer far greater selectivity than the immunoassay based screen. Of the 10 specimens that screened positive for amphetamines using immunoassay, only 1 specimen was confirmed positive representing a 90\% false positive rate. Of the 13 specimens that screened positive by immunoassay for cocaine, only four were confirmed positive representing a $69 \%$ false positive rate. When screened using DESI- 
MS/MS the false positive rates for amphetamines and cocaine were drastically reduced to $67 \%$ and $0 \%$, respectively. The enhanced selectivity afforded by the mass spectrometry based screen significantly reduced the number of positive screens. Only 3 of the 21 specimens screened positive for amphetamines using the DESI-MS/MS method compared to 10 positives seen with the immunoassay based screen (Table 59 and 60). Similarly, only 4 of the 21 specimens screened positive for cocaine using the DESI-MS/MS method compared to 13 positive seen with the immunoassay technique (Table 59 and 60). Of the 3 samples that screened positive for amphetamines using DESI-MS/MS, one was confirmed positive. All four specimens that screened positive for cocaine using DESI-MS/MS were confirmed positive. Sensitivity of the DESI-MS/MS method for amphetamine and methamphetamine in the meconium matrix was quite poor making identification of positive specimens difficult. Amphetamine and methamphetamine signal intensities in positive control specimens were not dissimilar to the signals observed in negative specimens (Figure 62). Regions between integrated signals in Figure 62 represent baseline signal. Amphetamine and methamphetamine signal intensities in positive control specimens were approximately equal to the baseline signal making the identification of positive samples extremely challenging. 


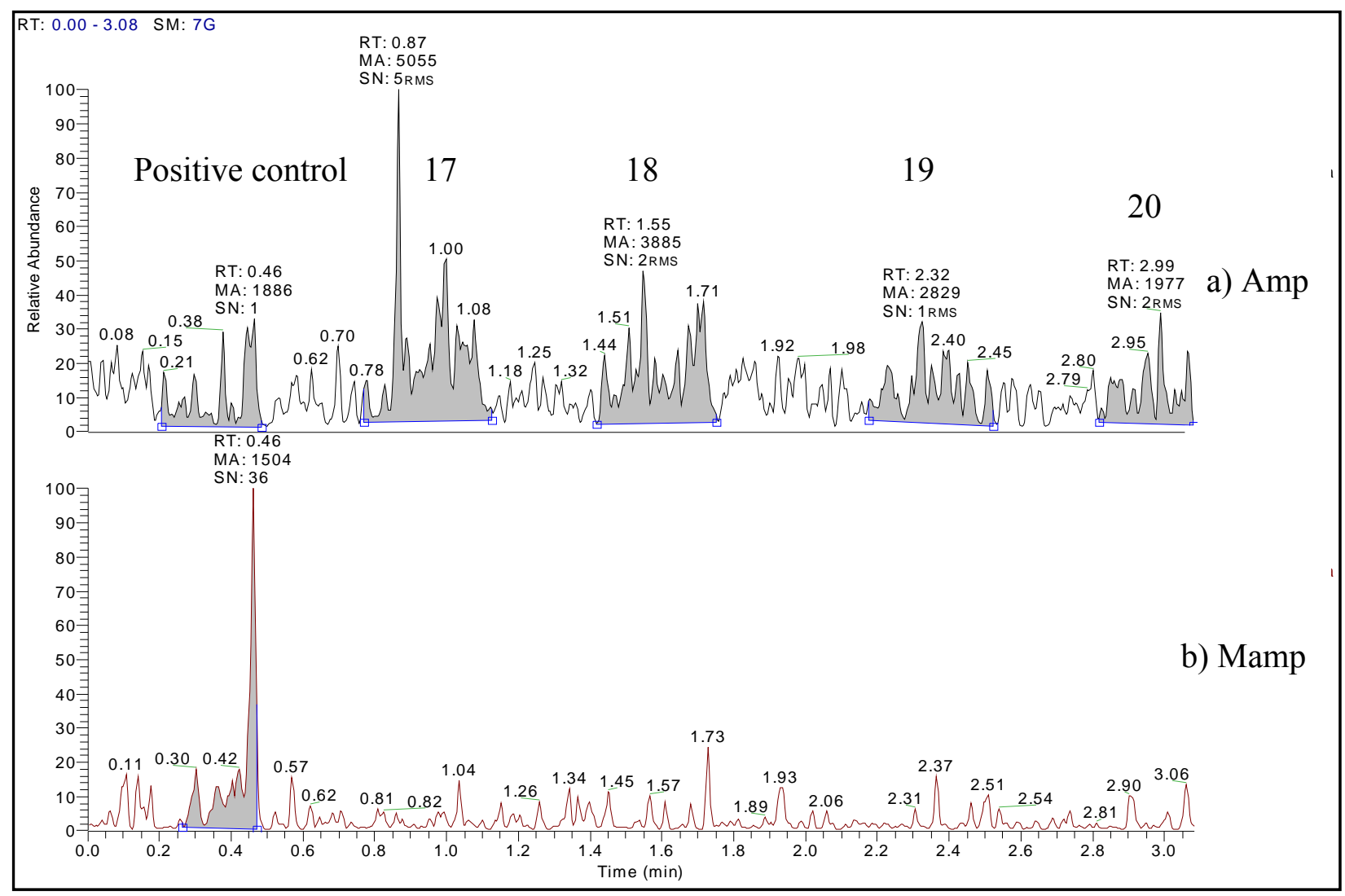

Figure 62: Show the mass spectral responses for amphetamine (a) and methamphetamine (b) in the positive quality control and four negative specimens. Signal intensities for the positive controls were not dissimilar to those of negative specimens.

Signal intensities for cocaine and benzoylecgonine in positive specimens were significantly greater than those observed for positive quality control specimens, allowing for easy identification (Figure 63). The signal to noise ratio for cocaine and benzoylecgonine in both positive quality control specimens and authentic specimens was far greater than those observed for the amphetamines. This increase in the $\mathrm{S} / \mathrm{N}$ ratio made identification of the cocaine derivatives much easier than the amphetamines. The sensitivity of the DESI-MS/MS method for cocaine and benzoylecgonine is encouraging and indicates that accurate and reliable screening is possible following limited sample preparation. 


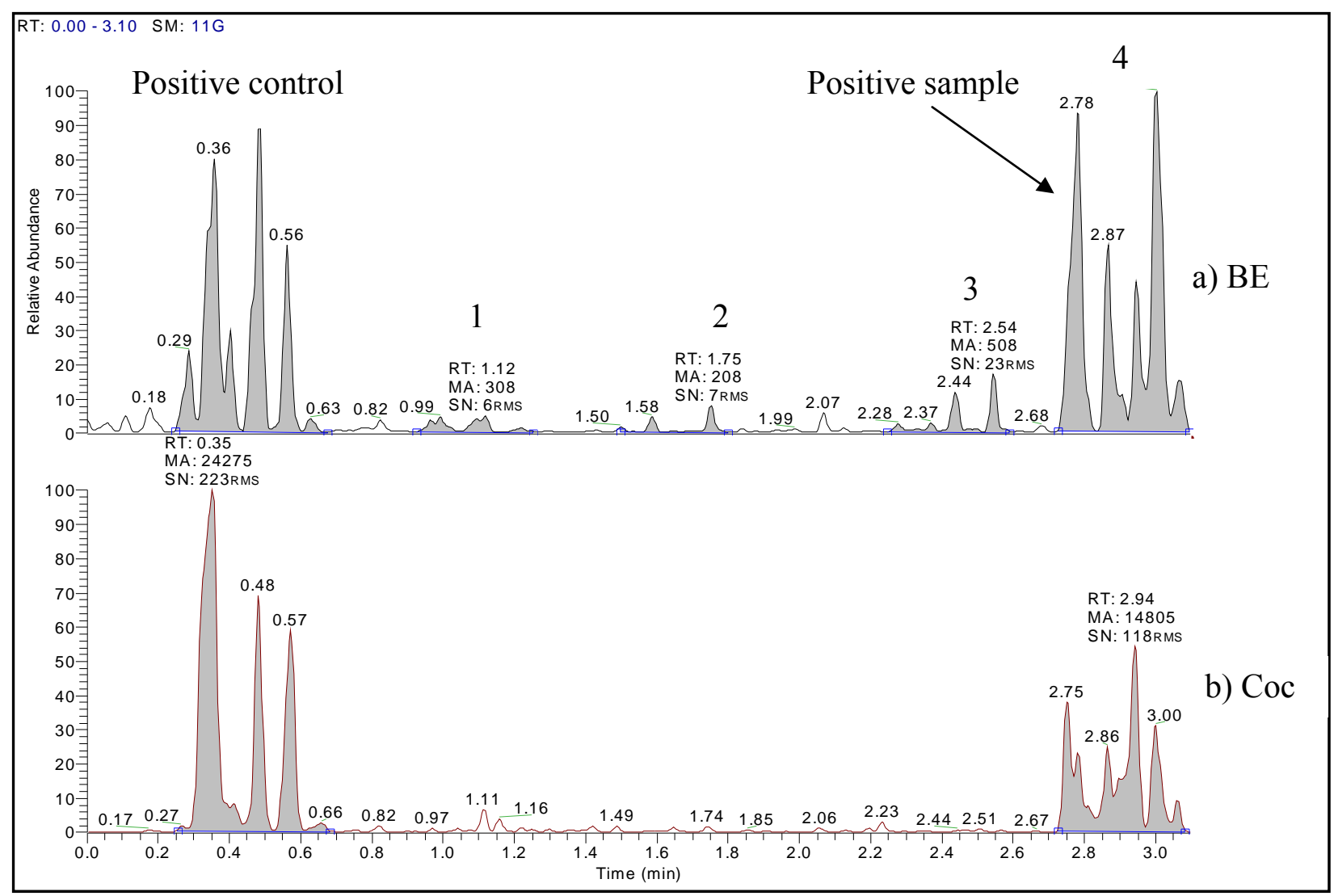

Figure 63: Shows the mass spectral responses for benzoylecgonine (a) and cocaine (b) in a positive quality control specimen, three negative specimens $(1,2$, and 3$)$ and a true positive specimen (4). Intense analyte signals in all true positives allowed for easy identification.

Studies indicate that mass spectrometry based screening techniques, such as the DESI-MS/MS method, could greatly reduce the number of false positive samples and prove more cost effective than traditional immunoassay techniques. The increased selectivity of the DESI-MS/MS screen is hardly surprising considering mass spectrometry has long been employed for confirmatory testing following a positive immunoassay screen. Confirmation analysis requires enhanced selectivity for the purpose of accurately identifying and quantifying individual analytes following a presumptive positive. The potential advantages of mass spectrometry based screening techniques have long been recognized, however, due to extensive sample preparation 
requirements, and cost of analysis, mass spectrometry is generally not well suited to high throughput screening. DESI-MS/MS appears to be more suited to high throughput screening as samples can be analyzed under ambient conditions following minimal sample preparation. While further studies are required to evaluate the sensitivity of DESI-MS/MS relative to immunoassay techniques, the methodology appears to offer superior selectivity without the need for extensive sample preparation. Such techniques would prove useful for the analysis of alternate biological matrices that have traditionally required significant sample preparation prior to instrumental analysis.

\subsection{Conclusions}

While further studies are required to evaluate the suitability of the DESI-MS/MS technique for the detection of analytes other than cocaine and amphetamine derivatives, preliminary studies indicate that the method may be well suited to the high throughput screening of meconium samples. Implementation of mass spectrometry based screening techniques such as DESI-MS/MS will greatly enhance screening selectivity and reduce the number of false positive results commonly observed with immunoassay based techniques.

Immunoassay screening techniques are well suited to the analysis of traditional biological specimens such as serum and urine, however, these techniques are limited in their capability to accurately identify target analytes in more complex matrices such as meconium. Furthermore, the turbid nature of meconium specimens often means that significant sample preparation is required before the matrix can be analyzed using immunoassay screening techniques. Development of a DESI-MS/MS method for screening meconium specimens aimed to alleviate existing problems surrounding immunoassay analysis by increasing screening selectivity and allowing for ambient sampling of meconium specimens following limited sample 
preparation. When applied to the analysis of 21 authentic meconium specimens, the DESIMS/MS method significantly reduced the rate of false positives compared to immunoassay analysis and allowed for the accurate identification authentic meconium specimens containing cocaine, benzoylecgonine, and amphetamines. 


\section{References}

1. $\quad$ World Drug Report 2000. 2000: Oxford. p. 1-172.

2. Results from the 2006 National Survey on Drug Use and Health: National Findings. 2006, Substance Abuse and Mental Health Services Administration.

3. Bar-Oz, B., et al., Comparison of meconium and neonatal hair analysis for detection of gestational exposure to drugs of abuse. Arch Dis Child Fetal Neonatal Ed, 2008. 88(2): p. F98-F100.

4. Rosen, T.S. and D.A. Bateman, Infants of addicted mothers. In Neonatal-Perinatal Medicine. 2001, Mosby: St Louis, MO. p. 661-673.

5. Koren, G., J. Hutson, and J. Gareri, Novel methods for the detection of drug and alcohol exposure during pregnancy: Implications for maternal and child health. Clin Pharmacol Ther, 2008. 83(4): p. 631-634.

6. Ostrea, E.M. and C.J. Chavez, Perinatal problems (excluding neonatal withdrawal) in maternal drug addiction: a study of 830 cases. J Pediatr, 1979. 94(2): p. 292-295.

7. Chasnoff, I.J., Drug use and women: establishing a standard of care. Ann N Y Acad Sci, 1989. 562: p. 208-210.

8. Ostrea, E.M., et al., Drug screening of newborns by meconium analysis: a large-scale, prospective, epidemiologic study. Pediatrics, 1992. 89(1): p. 107-113.

9. Lester, B.M., et al., The Maternal Lifestyle Study: drug use by meconium toxicology and maternal self-report. Pediatrics, 2001. 107(2): p. 309-317.

10. Forman, R., et al., Maternal and neonatal characteristics following exposure to cocaine in Toronto. Reprod Toxicol, 1993. 7(6): p. 619-622.

11. Birchfield, M., J. Scully, and A. Handler, Perinatal screening for illicit drugs: policies in hospitals in a large metropolitan area. J Perinatol, 1995. 15(3): p. 208-214.

12. Volpe, J.J., Effect of cocaine use on the fetus. N Engl J Med, 1992. 327(6): p. 399-407.

13. Chasnoff, I.J., et al., Cocaine / polydrug use in pregnancy: Two-year follow up. Pediatrics, 1992. 89: p. 284-289.

14. Fried, P.A. and B. Watkinson, 36- and 48-month neurobehavioral follow-up of children prenatally exposed to marijuana, cigarettes, and alcohol. J Dev Behav Pediatr, 1990. 11(2): p. 49-58.

15. Plessinger, M.A., Prenatal exposure to amphetamines. Risks and adverse outcomes in pregnancy. Obstet Gynecol Clin North Am, 1998. 25(1): p. 119-138.

16. Tabor, B.L., T. Smith-Wallace, and M.L. Yonekura, Perinatal outcome associated with PCP versus cocaine use. Am J Drug Alcohol Abuse, 1990. 16(3-4): p. 337-348.

17. Gillogley, K.M., et al., The perinatal impact of cocaine, amphetamine, and opiate use detected by universal intrapartum screening. Am J Obstet Gynecol, 1990. 163(5 pt 1): p. $1535-1542$.

18. Chasnoff, I.J. and D.R. Griffith, Cocaine: clinical studies of pregnancy and the newborn. Ann N Y Acad Sci, 1989. 562: p. 260-266.

19. Addis, A., et al., Fetal effects of cocaine: an updated meta-analysis. Reprod Toxicol, 2001. 15(4): p. 341-369.

20. Chasnoff, I.J., et al., Perinatal cerebral infarction and maternal cocaine use. J Pediatr, 1986. 108(3): p. 456-459.

21. Lopez, S.L., et al., Time of onset of necrotizing enterocolitis in newborn infants with known prenatal cocaine exposure. Clin Pediatr (Phila), 1995. 34(8): p. 424-429. 
22. Chasnoff, I.J., et al., Cocaine and pregnancy: clinical and toxicological implications for the neonate. Clin Chem, 1989. 35(7): p. 1276-1278.

23. Ryan, L., S. Ehrlich, and L. Finnegan, Cocaine abuse in pregnancy: effects on the fetus and newborn. Neurotoxicol Teratol, 1987. 9(4): p. 295-299.

24. Fulroth, R., B. Phillips, and D.J. Durand, Perinatal outcome of infants exposed to cocaine and/or heroin in utero. Am J Dis Child, 1989. 143(8): p. 905-910.

25. Franck, L. and J. Vilardi, Assessment and management of opioid withdrawal in ill neonates. Neonatal Netw, 1995. 14(2): p. 39-48.

26. Moore, C., A. Negrusz, and D. Lewis, Determination of drugs of abuse in meconium. J Chromatogr B Biomed Sci Appl, 1998. 713(1): p. 137-146.

27. Soo, V.A., Neonatal Drug Testing, in Advance for medical laboratory professionals. 2000. p. 19-23.

28. Araojo, R., S. McCune, and K. Feibus, Substance abuse in pregnant women: Making improved detection a good clinical outcome. Clin Pharmacol Ther, 2008. 83(4): p. 520522.

29. Administration, T.I.D.o., RFP-5-56 Section one: General information and requested products/services. 2004.

30. Marin, J.J.G., O. Briz, and M.A. Serrano, A Review on the Molecular Mechanisms Involved in the Placental Barrier for Drugs. Current Drug Delivery, 2004. 1(3): p. 275289.

31. Ganapathy, V., et al., Drugs of abuse and placental transport. Advanced Drug Delivery Reviews 1, 1999. 38(1): p. 99-110.

32. Gedeon, C. and G. Koren, Designing pregnancy centered medications: drugs which do not cross the human placenta. Placenta, 2006. 27(8): p. 861-868.

33. Heikkilä, A., O.V. Renkonen, and R. Erkkola, Pharmacokinetics and transplacental passage of imipenem during pregnancy. Antimicrob Agents Chemother, 1992. 36(12): p. 2652-2655.

34. Huestis, M.A. and R.E. Choo, Drug abuse's smallest victims: in utero drug exposure. Forensic Sci Int, 2002. 128(1-2): p. 20-30.

35. Minozzi, S., et al., Maintenance agonist treatments for opiate dependent pregnant women. Cochrane Database Syst Rev, 2008(2).

36. Weier, N., et al., Placental drug disposition and its clinical implications. Curr Drug Metab, 2008. 9(2): p. 106-121.

37. Nanovskaya, T.N., et al., Transfer of methadone across the dually perfused preterm human placental lobule. Am J Obstet Gynecol, 2008. 198(1): p. 126.e1-4.

38. Klieger, C., E. Pollex, and G. Koren, Treating the mother - protecting the unborn: The safety of hypoglycemic drugs in pregnancy. J Matern Fetal Neonatal Med, 2008. 21(3): p. 191-196.

39. Ostrea, E.M., Understanding drug testing in the neonate and the role of meconium analysis. J Perinat Neonatal Nurs, 2001. 14(4): p. 61-82.

40. Schonberg, S.K., Substance Abuse: A guide for health professionals. 1988, Elk Grove Village, IL: American Academy of Pediatrics and Center for Advanced Health Studies.

41. Halstead, A.C., et al., Timing of specimen collection is crucial in urine screening of drug dependent mothers and newborns. Clin Biochem, 1988. 21(1): p. 59-61.

42. Mandatory guidelines for federal workplace drug testing programs. 1988, Fed Regist. p. 11979-11989. 
43. Hicks, J.M., A. Morales, and S.J. Soldin, Drugs of abuse in a pediatric outpatient population. Clin Chem, 1990. 36(6): p. 1256-1257.

44. Casanova, O.Q., et al., Detection of cocaine exposure in the neonate. Analyses of urine, meconium, and amniotic fluid from mothers and infants exposed to cocaine. Arch Pathol Lab Med, 1994. 118(10): p. 988-993.

45. Ostrea, E.M., et al., Drug screening of meconium in infants of drug-dependent mothers: an alternative to urine testing. J Pediatr, 1989. 115(3): p. 474-477.

46. Moore, C.M., et al., The detection of hydrocodone in meconium: two case studies. J Anal Toxicol, 1995. 19(6): p. 514-518.

47. Callahan, C.M., et al., Measurement of gestational cocaine exposure: sensitivity of infants' hair, meconium, and urine. J Pediatr, 1992. 120(5): p. 763-768.

48. Ryan, R.M., et al., Meconium analysis for improved identification of infants exposed to cocaine in utero. J Pediatr, 1994. 125(3): p. 435-440.

49. Gourley, G.R., B. Kreamer, and R. Arend, Excremental studies in human neonates. Identification of zinc coproporphyrin as a marker for meconium. Gastroenterology, 1990. 99(6): p. 1705-1709.

50. Woods, J.R., "Significance of amniotic fluid meconium," Maternal fetal medicine: Principles and Practice. 1984, Philadelphia, PA: W.B Saunders.

51. Gallardo, E. and J.A. Queiroz, The role of alternative specimens in toxicological analysis. Biomedical Chromatography, 2008. 22: p. 795-821.

52. Browne, S.P., et al., Analysis of meconium for cocaine in neonates. J Chromatogr B Analyt Technol Biomed Life Sci, 1992. 575(1): p. 158-161.

53. Ostrea, E.M., P.M. Parks, and M.J. Brady, Rapid isolation and detection of drugs in meconium of infants of drug-dependent mothers. Clin Chem, 1988. 34(11): p. 2372-2373.

54. Maynard, E.C., L.P. Amoruso, and W. Oh, Meconium for drug testing. Am J Dis Child, 1991. 145(6): p. 650-652.

55. Ramage, H., A spectrographic analysis of the metallic content of meconium. Biochem J, 1933. 27(3): p. 674-677.

56. Rapoport, S. and D.J. Buchanan, The composition of Meconium; isolation of bloodgroup-specific polysaccharides; abnormal compositions of meconium in meconium ileus. Science, 1950. 112(2901): p. 150-153.

57. Emery, J.L., The tryptic activity and presence of cornified squames in meconium as a diagnostic aid in congenital intestinal obstruction. Arch Dis Child, 1952. 27(131): p. 6772.

58. Shahani, R.T., N. Shah, and S.S. Sabnis, Studies in immunization using blood group substances prepared from meconium. Indian J Pathol Bacteriol, 1961. 4: p. 214-226.

59. Kinsella, R.A.J., et al., Enteric excretion of metabolites of steroid hormones in the human. I. Isolation of estriol from meconium. J Biol Chem, 1956. 219(1): p. 265-271.

60. Gsell-Busse, M.A., Arch. exp. Path. u. Pharmakol., 1929. 139: p. 328.

61. Francis, F.E., N.C. Shen, and R.A.J. Kinsella, Enteric excretion of metabolites of steroid hormones in the human. II. Isolation of delta 5-androsten-3 beta-ol-17-one from meconium. J Biol Chem, 1960. 235: p. 1957-1959.

62. Menini, E. and E. Dicfalusy, Isolation and identification of estriol glucosiduronate in human meconium. Endocrinology, 1960. 67: p. 500-510.

63. Menini, E. and E. Dicfaluzy, Isolation and identification of sodium estriol-3-sulphate in human meconium. Endocrinology, 1961. 68(492-500): p. 492. 
64. Francis, F.E., N.H. Shen, and R.A.J. Kinsella, Enteric excretion of metabolites of steroid hormones in the human. III. Isolation of 3 alpha-hydroxy-5 beta-pregn-16-en-20-one (16pregnenolone) from meconium. Biochemistry, 1962. 1: p. 1184-1186.

65. Francis, F.E. and R.A.J. Kinsella, Enteric excretion of metabolites of steroid hormones in the human subject. IV. Isolation of 5-beta-pregnane-3-alpha, 20-alpha-diol from meconium. J Clin Endocrinol Metab, 1966. 26(2): p. 128-132.

66. Green, M.N. and H. Shwachman, Presumptive tests for cystic fibrosis based on serum protein in meconium. Pediatrics, 1968. 41(5): p. 989-992.

67. Luppi, A. and M. Iannaci, On the detection of Listeria monocytogenes in meconium. Preliminary note. G Mal Infett Parassit, 1966. 18(8): p. 562-563.

68. Miettinen, T.A. and T. Luukkainen, Gas-liquid chromatographic and mass spectrometric studies on sterols in vernix caseosa, amniotic fluid and meconium. Acta Chem Scand, 1968. 22(8): p. 2603-2612.

69. Ostrea, E.M.J., et al., The detection of heroin, cocaine and cannabinoid metabolites in the stools of infants of drug dependant mothers: clinical significance. Pediatr Res, 1987. 21(240).

70. Maynard, E., L. Amoruso, and W. Oh, Meconium for drug testing. Am J Dis Child, 1991. 145(6): p. 650-652.

71. Ostrea, E.M.J. and R.A. Welch, Detection of prenatal drug exposure in the pregnant women and her newborn infant. Clin Perinatol, 1991. 18(3): p. 629-645.

72. Dahlem, P., et al., Detection of drugs in meconium. Monatsschr Kinderheilkd, 1992. 140(6): p. 354-356.

73. Yawn, B.P., R.A. Yawn, and D.L. Uden, Substance abuse in rural Midwestern pregnant women. Arch Fam Med, 1992. 1(1): p. 83-88.

74. Dusick, A.M., et al., Risk of intracranial hemorrhage and other adverse outcomes after cocaine exposure in a cohort of 323 very low birth weight infants. J Pediatr, 1993. 122(3): p. 438-445.

75. Rosengren, S.S., et al., Meconium testing for cocaine metabolite: prevalence, perceptions, and pitfalls. Am J Obstet Gynecol, 1993. 168(5): p. 1449-1456.

76. Lombardero, N., et al., Measurement of cocaine and metabolites in urine, meconium, and diapers by gas chromatography/mass spectrometry. Ann Clin Lab Sci, 1993. 23(5): p. 385-394.

77. Wingert, W.E., et al., A comparison of meconium, maternal urine and neonatal urine for detection of maternal drug use during pregnancy. J Forensic Sci, 1994. 39(1): p. 150158.

78. Yawn, B.P., et al., Prenatal drug use in Minneapolis-St Paul, Minn. A 4-year trend. Arch Fam Med, 1994. 3(6): p. 520-527.

79. Moriya, F., et al., Testing for Drugs of Abuse in Meconium of Newborn Infants. Journal of Analytical Toxicology, 1994. 18(1): p. 41-45.

80. Fox, C.H., Cocaine use in pregnancy. J Am Board Fam Pract, 1994. 7(3): p. 225-228.

81. DiGregorio, G.J., et al., Determination of cocaine usage in pregnant women by a urinary EMIT drug screen and GC-MS analyses. J Anal Toxicol, 1994. 18(5): p. 247-250.

82. Franssen, R.M.E., et al., Analysis of morphine and amphetamine in meconium with immunoassay and HPLC-diode-array detection. J Anal Toxicol, 1994. 18(5): p. 294-295.

83. Browne, S., et al., Detection of cocaine, norcocaine, and cocaethylene in the meconium of premature neonates. J Forensic Sci, 1994. 39(6): p. 1515-1519. 
84. Bibb, K.W., et al., Drug screening in newborns and mothers using meconium samples, paired urine samples, and interviews. J Perinatol, 1995. 15(3): p. 199-202.

85. Moriya, F., et al., Detection of drugs-of-abuse in meconium of a stillborn baby and in stool of a deceased 41-day-old infant. J Forensic Sci, 1995. 40(3): p. 505-508.

86. Goosensen, M., L.M. Stolk, and B.J. Smit, Analysis of 11-nor-delta-9-THC-carboxylic acid in meconium with immunoassay and HPLC diode-array detection. J Anal Toxicol, 1995. 19(5): p. 330.

87. Oyler, J., et al., Cocaine disposition in meconium from newborns of cocaine-abusing mothers and urine of adult drug users. J Anal Toxicol, 1996. 20(6): p. 453-462.

88. Kwong, T.C. and R.M. Ryan, Detection of intrauterine illicit drug exposure by newborn drug testing. National Academy of Clinical Biochemistry. Clin Chem, 1997. 43(1): p. 235242.

89. Pichini, S., et al., Quantification of arecoline (areca nut alkaloid) in neonatal biological matrices by high-performance liquid chromatography/electrospray quadrupole mass spectrometry. Rapid Commun Mass Spectrom, 2003. 17(17): p. 1958-1964.

90. Choo, R.E., et al., Determination of methadone, 2-ethylidene-1,5-dimethyl-3,3diphenylpyrrolidine, 2-ethyl-5-methyl-3,3-diphenylpyraline and methadol in meconium by liquid chromatography atmospheric pressure chemical ionization tandem mass spectrometry. J Chromatogr B Analyt Technol Biomed Life Sci, 2005. 814(2): p. 369373.

91. Kato, K., et al., Quantifying Phthalate Metabolites in Human Meconium and Semen Using Automated Off-Line Solid-Phase Extraction Coupled with On-Line SPE and Isotope-Dilution High-Performance Liquid Chromatography-Tandem Mass Spectrometry. Analytical Chemistry, 2006. 78(18): p. 6651-6655.

92. Coles, R., et al., Simultaneous Determination of Codeine, Morphine, Hydrocodone, Hydromorphone, Oxycodone, and 6-Acetylmorphine in Urine, Serum, Plasma, Whole Blood, and Meconium by LC-MS-MS. Journal of Analytical Toxicology, 2007. 31(1): p. $1-14$.

93. Kelly, T., T.R. Gray, and M.A. Huestis, Development and validation of a liquid chromatography-atmospheric pressure chemical ionization-tandem mass spectrometry method for simultaneous analysis of 10 amphetamine-, methamphetamine- and 3,4methylenedioxymethamphetamine-related (MDMA) analytes in human meconium. J Chromatogr B Analyt Technol Biomed Life Sci, 2008. 867(2): p. 194-204.

94. Marin, S.J., R.M. Coles, M, and G.A. McMillin, Quantitation of Benzodiazepines in Urine, Serum, Plasma, and Meconium by LC-MS-MS. J Anal Toxicol, 2008. 32(7): p. 491-498.

95. Gray, T.R., D.M. Shakleya, and M.A. Huestis, Quantification of nicotine, cotinine, trans3'-hydroxycotinine, nornicotine and norcotinine in human meconium by liquid chromatography/tandem mass spectrometry. J Chromatogr B Analyt Technol Biomed Life Sci, 2008. 863(1): p. 107-114.

96. Kacinko, S.L., D.M. Shakleya, and M.A. Huestis, Validation and application of a method for the determination of buprenorphine, norbuprenorphine, and their glucuronide conjugates in human meconium. Anal Chem, 2008. 80(1): p. 246-252.

97. Haram-Mourabet, S., R.G. Harper, and R.A. Wapnir, Mineral composition of meconium. Journal of the american college of nutrition, 1998. 17(4): p. 356-360. 
98. Villanueva, M.E., et al., Detection of cytomegalovirus in the meconium of infected newborns by polymerase chain reaction. Infect Dis Obstet Gynecol, 2000. 8(3-4): p. 166171.

99. Ortega García, J.A., et al., Meconium and neurotoxicants: searching for a prenatal exposure timing. Arch Dis Child, 2006. 91(8): p. 642-646.

100. Karch, S.K., Postmortem toxicology of abused drugs. 2008, Boca Raton: CRC Press.

101. Shibamoto, T., Liquid chromatographic analysis. 1994, CRC Press.

102. Skoog, D.A., Principles of Instrumental Analysis. 3rd ed. 1985: Saunders College Publishing.

103. Negrusz, A., S. Jickells, and S. Dawling, Gas Chromatography, in Clarke's Analytical Forensic Toxicology, S. Jickell and A. Negrusz, Editors. 2008, Pharmaceutical Press. p. 469-511.

104. Van Deemter, J.J., F.J. Zuiderweg, and A. Klingengerg, J. Chem. Eng. Sci, 1956. 5: p. 272.

105. Neue, U.D., HPLC Columns: Theory, Technology and Practice. 1997, New York: Wiley$\mathrm{VCH}$.

106. Meloan, C.E., Chemical separation principles, in Techniques and Experiments. 1999, John Wiley \& Sons: Canada.

107. Swartz, M.E., Ultra Performance Liquid Chromatography (UPLC): An Introduction. Separation Science Redefined, 2005: p. 8-14.

108. Ultra Performance $L C^{T M}$ by design, 2004. Waters Corporation, USA, 720000880EN $L L \& L W-U L$.

109. Heftmann, E., Chromatography. 1961, New York: Reinhold Publishing Corporation.

110. Acquity Ultraperformance LC by design, 2007. Waters Corporation, USA, 720000880EN $L B-A C$.

111. Sherma, J., UPLC: Ultra-performance liquid chromatography. J AOAC Int, 2005. 88(3): p. 63A-67A.

112. Ultra Performance LC Separation Science Redefined, 2004. Waters Corporation, USA, 720001136EN.

113. MacNair, J.E., K.C. Lewis, and J.W. Jorgenson, Ultrahigh-Pressure Reversed-Phase Liquid Chromatography in Packed Capillary Columns. Anal Chem, 1997. 69(6): p. 983989.

114. Henry, C.M., Kicking Separation up a Notch. C\&EN, 2004. 82(47): p. 68-71.

115. Plumb, R., et al., Ultra-performance liquid chromatography coupled to quadrupoleorthogonal time-of-flight mass spectrometry. Rapid Commun Mass Spectrom, 2004. 18(19): p. 2331-2337.

116. Ho, C.S., et al., Electrospray ionization mass spectrometry: principles and clinical applications. Clin Biochem Rev, 2003. 24: p. 3-12.

117. McLafferty, F.W. and F. Turecek, Interpretation of mass spectra. 4th ed. 1993: Mill Valley: University science books.

118. Watson, J.T., Introduction to Mass Spectrometry. 1985, New York: Raven Press.

119. SOFT/AAFS, Society of Forensic Toxicologist and American Academy of Forensic Sciences: Forensic Toxicology laboratory guidelines 2006.

120. Maurer, H.H., Liquid chromatography-mass spectrometry in forensic and clinical toxicology. Journal of Chromatography B, 1998. 713: p. 3-25. 
121. Chung, A., Validation of an Ultra Performance Liquid Chromatography Tandem Mass Spectrometry(UPLC $\left.{ }^{\mathrm{TM}} / \mathrm{MS} / \mathrm{MS}\right)$ Method for Forensic Toxicological Analysis: Confirmation and Quantitation of Lysergic Acid Diethylamide (LSD) and its Congeners in Forensic Samples. 2006, University of Saskatchewan Saskatoon.

122. Lowinson, J.H., et al., Substance Abuse: A Comprehensive Textbook. 4th ed. 2004: Lippincott Williams \& Wilkins.

123. Careri, M., A. Mangia, and M. Musci, Applications of liquid chromatography-mass spectrometry interfacing systems in food analysis: pesticide, drug adn toxic substance residues. Journal of Chromatography A, 1996. 727: p. 153-184.

124. Hoja, H., et al., Applications of liquid chromatography-Mass spectrometry in analytical toxicology: A review. Journal of Analytical Toxicology, 1997. 21(2): p. 116-126.

125. Tal'roze, V.L., V.E. Skurat, and G.V. Karpov, 1969.

126. Niessen, W.M.A. and A.P. Tinke, Liquid chromatography-mass spectrometry General principles and instrumentation. Journal of Chromatography A, 1995. 703: p. 37-57.

127. van der Greef, J. and W.M.A. Neissen, Hyphenated methods in mass spectrometry. International journal of mass spectrometry and ion processes, 1992. 116: p. 857-873.

128. Burlingame, A.L., R.K. Boyd, and S.J. Gaskell, Mass Spectrometry. Anal. Chem, 1994. 66: p. 634R-683R.

129. Blakley, C.R. and M.L. Vestal, Thermospray interface for liquid chromatography/mass spectrometry. Anal. Chem, 1983. 55(750-754): p. 750.

130. Slobodnik, J., B.L.M. Van Baar, and U.A.T. Brinkman, Column liquid chromatographymass spectrometry: selected techniques in environmental applications for polar pesticides and related compounds. Journal of Chromatography A, 1995. 703: p. 81-121.

131. Gelpi, E., Biomedical and biochemical applications of liquid chromatography-mass spectrometry. Journal of Chromatography A, 1995. 703: p. 59-80.

132. Willoughby, R.C. and R.F. Browner, Monodisperse Aerosol Generation Interface for Combining Liquid Chromatography with Mass Spectroscopy. Anal. Chem, 1984. 56: p. 2625-2631.

133. Heller, D.N. and F.J. Schenck, Particle beam liquid chromatography/mass spectrometry with negative ion chemical ionization for the confirmation of ivermectin residue in bovine milk and liver. Biol Mass Spectrom, 1993. 22(3): p. 184-193.

134. Pacifici, R., et al., Nicotine, cotinine, and trans-3-hydroxycotinine levels in seminal plasma of smokers: effects on sperm parameters. Ther Drug Monit, 1993. 15(5): p. 358363.

135. Niessen, W.M.A., Advances in instrumentation in liquid chromatography-mass spectrometry and related liquid-introduction techniques. Journal of Chromatography A, 1998. 794: p. 407-435.

136. Niessen, W.M.A., State-of-the-art in liquid chromatography-mass spectrometry. Journal of Chromatography A, 1999. 856: p. 179-197.

137. Voyksner, R.D., Electrospray ionization mass spectrometry: Fundamentals, instrumentation and applications, R.B. Cole, Editor. 1997, Wiley: New York.

138. Guerrera, I.C. and O. Kleiner, application of mass spectrometry in proteomics. Bioscience reports, 2005. 25(1-2): p. 71-93.

139. Rosenberg, E., The potential of organic (electrospray- and atmospheric pressure chemical ionization) mass spectrometric techniques coupled to liquid-phase separation for speciation analysis. Journal of Chromatography A, 2003. 1000: p. 841-889. 
140. Mitchum, R.K., et al., Atmospheric pressure ionization mass spectrometry: the routine determination of 2,4,5-trichlorophenoxyacetic acid and its metabolites. Biomed Mass Spectrom, 1981. 8(11): p. 539-545.

141. Mitchum, R.K. and W.A. Korfmacher, Atmospheric pressure ionization mass spectrometry. Anal. Chem, 1983. 55: p. 1485A-1499A.

142. Byrdwell, W.C., Atmospheric pressure chemical ionization mass spectrometry for analysis of lipids. Lipids, 2001. 36: p. 327-346.

143. Bruins, A.P., Mechanistic aspects of electrospray ionization. Journal of Chromatography A, 1998. 794: p. 345-357.

144. Taflin, D.C., T.L. Ward, and E.J. Davis, Electrified droplet fission and the Rayleigh limit. Langmuir, 1989. 5: p. 376-384.

145. Dole, M., et al., Molecular beams of macroions. J Chem Phys, 1968. 49: p. 2240-2249.

146. Whitehouse, C.M., et al., Electrospray interface for liquid chromatographs and mass spectrometers. Anal. Chem, 1985. 57: p. 675-679.

147. Iribarne, J.V. and B.A. Thomson, On the evaporation of charged ions from small droplets. J Chem Phys, 1976. 64: p. 2287-2294.

148. Stephenson, J.L. and S.A. McLuckey, Ion/ion proton transfer reactions for protein mixture analysis. Anal. Chem, 1996. 68(22): p. 4026-4032.

149. Cech, N.B. and C.G. Enke, Practical implications of some recent studies in electrospray ionization fundamentals. Mass spectrometry reviews, 2001. 20: p. 362-387.

150. Kebarle, P., A brief overview of the present status of the mechanisms involved in electrospray mass spectrometry. J Mass Spectrom, 2000. 35(7): p. 804-817.

151. Amad, M.H., et al., Importance of gas-phase proton affinities in determining the electrospray ionization response for analytes and solvents. J Mass Spectrom, 2000. 35: p. 784-789.

152. Holcapek, M., L. Kolarova, and M. Nobilis, High performance liquid chromatographytandem mass spectrometry in the identification and determination of phase I and phase II drug metabolites. Anal Bioanal Chem, 2008. 391: p. 59-78.

153. Nobilis, M., et al., Identification and determination of phase II nabumetone metabolites by high-performance liquid chromatography with photodiode array and mass spectrometric detection. Journal of Chromatography A, 2004. 1031: p. 229-236.

154. de Hoffmann, E., Tandem mass spectrometry: a primer. Journal of mass spectrometry, 1996. 31: p. 129-137.

155. Griffiths, W.J., et al., Electrospray and tandem mass spectrometry in biochemistry. Biochem. J., 2001. 355: p. 545-561.

156. Yost, R.A. and C.G. Enke, Selected ion fragmentation with a tandem quadrupole mass spectrometer. Journal of the American Chemical Society, 1977. 100(7): p. 2274-2275.

157. Yang, Z. and S. Wang, Recent developments in application of high performance liquid chromatography-tandem mass spectrometry in therapeutic drug monitoring of immunosuppresants. Journal of Immunological methods, 2008. 336: p. 98-103.

158. Rossi, D.T., et al., Tandem-in-time mass spectrometry as a quantitative bioanalytical tool. Analytical Chemistry, 1997. 69(22): p. 4519-4523.

159. McMaster, M., LC/MS A practical user's guide. 2005, Wiley-Interscience: Hoboken, New Jersey.

160. March, R.E., An Introduction to Quadrupole Ion Trap Mass Spectrometry. Journal of mass spectrometry, 1997. 32: p. 351-369. 
161. Thomas, R., Practical Guide to ICP-MS 2003, CRC Press.

162. McLafferty, F.W., Tandem mass spectrometry, ed. F.W. McLafferty. 1983, New York: Wiley.

163. Waters TQ Detector Operators Guide, 2007. Waters Corporation, USA. (Revision B).

164. Waters TQ Detector Operator's Guide, 2007. Waters Corporation, USA.

165. Dominguesa, P., et al., Constant neutral loss scanning for the characterization of glycerol phosphatidylcholine phospholipids Journal of the American Society for Mass Spectrometry, 1998. 9(11): p. 1189-1195.

166. McLafferty, F.W., et al., Unknown identification using reference mass spectra. quality evaluation of databases Journal of the American Society for Mass Spectrometry, 1999. 10(12): p. 1229-1240.

167. Sauvage, F.L., et al., Screening of drugs and toxic compounds with liquid chromatography-linear ion trap tandem mass spectrometry. Clin Chem, 2006. 52(9): p. 1735-1742.

168. Herrin, G.L., H.H. McCurdy, and W.H. Wall, Investigation of an LC-MS-MS (QTrap) method for the rapid screening and identification of drugs in postmortem toxicology whole blood samples. J Anal Toxicol, 2005. 29(7): p. 599-606.

169. Marquet, P., et al., Comparison of a preliminary procedure for the general unknown screening of drugs and toxic compounds using a quadrupole-linear ion-trap mass spectrometer with a liquid chromatography-mass spectrometry reference technique. J Chromatogr B, 2003. 789: p. 9-18.

170. Miyagi, H., et al., [Improvement of a multi-target screening analysis for drugs using a QTRAP liquid chromatography/tandem mass spectrometry system]. Rinsho Byori, 2007. 55(4): p. 309-318.

171. Quattro Premier ${ }^{\mathrm{TM}}$ : ZSpray ${ }^{\mathrm{TM}}$ Robustness, 2004. Waters Corporation, USA, 720000916EN.

172. Herbert, C.G., R. Alexander, and W. Johnstone, Mass Spectrometry Basics. 2002: CRC Press.

173. Kruger, T.L., et al., Mixture analysis by mass-analyzed ion kinetic energy spectrometry. Anal Chem, 1976. 48(14): p. 2113-2119.

174. Hunt, D.F., J. Shabanowitz, and A.B. Giordani, Collision Activated Decompositions of Negative Ions in Mixture Analysis with a Triple Quadrupole Mass Spectrometer. Anal Chem, 1980. 52: p. 386-390.

175. Chakel, J.A., C.A. Myerholtz, and C.G. Enke, paper presented as ASMS meeting. Minneapolis, May, 1981.

176. Verweij, A.M., M.L. Hordijk, and P.J. Lipman, Quantitative liquid chromatographic thermospray-tandem mass spectrometric analysis of some analgesics and tranquilizers of the methadone, butyrophenone, or diphenylbutylpiperidine groups in whole blood. J Anal Toxicol, 1995. 19(2): p. 65-68.

177. Verweij, A.M., M.L. Hordijk, and P.J. Lipman, Liquid chromatographic-thermospray tandem mass spectrometric quantitative analysis of some drugs with hypnotic, sedative and tranquillising properties in whole blood. Journal of Chromatography B, 1996. 686: p. 27-34.

178. Cai, J. and J. Henion, On-line immunoaffinity extraction-coupled column capillary liquid chromatography/tandem mass spectrometry: trace analysis of LSD analogs and metabolites in human urine. Anal Chem, 1996. 68(1): p. 72-78. 
179. Cai, J. and J. Henion, Elucidation of LSD in vitro metabolism by liquid chromatography and capillary electrophoresis coupled with tandem mass spectrometry. J Anal Toxicol, 1996. 20(1): p. 27-37.

180. Constanzer, M., C. Chavez, and B. Matuszewski, Development and comparison of highperformance liquid chromatographic methods with tandem mass spectrometric and ultraviolet absorbance detection for the determination of

cyclobenzaprine in human plasma and urine. Journal of chromatography B, 1995. 666: p. 117126.

181. Kleinschnitz, M., M. Herderich, and P. Schreier, Determination of 1,4-benzodiazepines by high-performance liquid chromatography-electrospray tandem mass spectrometry. Journal of Chromatography B, 1996. 676: p. 61-67.

182. Maurer, H.H., Current role of liquid chromatography-mass spectrometry in clinical and forensic toxicology. Anal Bioanal Chem, 2007. 388: p. 1315-1325.

183. Hegstad, S., et al., Drug screening of hair by liquid chromatography-tandem mass spectrometry. J Anal Toxicol, 2008. 32(5): p. 364-372.

184. Concheiro, M., et al., Determination of illicit and medicinal drugs and their metabolites in oral fluid and preserved oral fluid by liquid chromatography-tandem mass spectrometry. Anal Bioanal Chem, 2008. 391(6): p. 2329-2338.

185. Gaulier, J.M., et al., Identification of acepromazine in hair: an illustration of the difficulties encountered in investigating drug-facilitated crimes. J Forensic Sci, 2008. 53(3): p. 755-759.

186. Ojanperä, I., et al., An epidemic of fatal 3-methylfentanyl poisoning in Estonia. Int J Legal Med, 2008.

187. Roman, M., et al., Quantitation of seven low-dosage antipsychotic drugs in human postmortem blood using LC-MS-MS. J Anal Toxicol, 2008. 32(2): p. 147-155.

188. Liu, W., et al., Simultaneous screening for 22 poisonous alkaloids in blood by liquid chromatography-tandem mass spectrometry with multiple-reaction monitoring. Fa Yi Xue Za Zhi, 2007. 23(5): p. 349-352.

189. Concheiro, M., et al., Determination of illicit drugs and their metabolites in human urine by liquid chromatography tandem mass spectrometry including relative ion intensity criterion. J Anal Toxicol, 2007. 31(9): p. 573-580.

190. Castaing, N., et al., Quantification of eight new antidepressants and five of their active metabolites in whole blood by high-performance liquid chromatography-tandem mass spectrometry. J Anal Toxicol, 2007. 31(6): p. 334-341.

191. de Castro, A., et al., High-throughput on-line solid-phase extraction-liquid chromatography-tandem mass spectrometry method for the simultaneous analysis of 14 antidepressants and their metabolites in plasma. J Chromatogr A, 2007. 1160(1-2): p. 312.

192. Quintela, O., et al., Liquid chromatography-tandem mass spectrometry for detection of low concentrations of 21 benzodiazepines, metabolites, and analogs in urine: method with forensic applications. Clin Chem, 2006. 52(7): p. 1346-1355.

193. Ballard, K.D., et al., An analytical strategy for quaternary ammonium neuromuscular blocking agents in a forensic setting using LC-MS/MS on a tandem quadrupole/time-offlight instrument. J Am Soc Mass Spectrom, 2006. 17(10): p. 1456-1468. 
194. Frison, G., et al., Quantification of citalopram or escitalopram and their demethylated metabolites in neonatal hair samples by liquid chromatography-tandem mass spectrometry. Ther Drug Monit, 2008. 30(4): p. 467-473.

195. Favretto, D., et al., Potentials of ion trap collisional spectrometry for liquid chromatography/electrospray ionization tandem mass spectrometry determination of buprenorphine and nor-buprenorphine in urine, blood and hair samples. Rapid Commun Mass Spectrom, 2006. 20(8): p. 1257-1265.

196. Gergov, M., I. Ojanperä, and E. Vuori, Simultaneous screening for 238 drugs in blood by liquid chromatography-ionspray tandem mass spectrometry with multiple reaction monitoring. J Chromatogr B, 2003. 795: p. 41-53.

197. Mueller, C.A., et al., Development of a multi-target screening analysis for 301 drugs using a QTrap liquid chromatography/tandem mass spectrometry system and automated library searching. Rapid Commun Mass Spectrom, 2005. 19: p. 1332-1338.

198. Peters, F.T., O.H. Drummer, and F. Musshoff, Validation of new methods. Forensic Science International, 2007. 165: p. 216-224.

199. FDA, Guidance for Industry Bioanalytical method validation. 2001.

200. Shabir, G.A., Validation of high-performance liquid chromatography methods for pharmaceutical analysis Understanding the differences and similarities between validation requirements of the US fodd and drug administration, the US pharmacopeia and the international conference on harmonization. Journal of Chromatography A, 2003. 987: p. 57-66.

201. Shah, V.P., et al., Bioanalytical method validation - A revisit with a decade of progress. Pharmaceutical research, 2000. 17(12): p. 1551-1557.

202. Boulanger, B., et al., An analysis of the SFSTP guide on validation of chromatographic bioanalytical methods: progresses and limitations. Journal of Pharmaceutical and Biomedical Analysis, 2003. 32: p. 753-765.

203. Armbruster, D.A., M.D. Tillman, and L.M. Hubbs, Limit of detection (LOD)/limit of quantitation (LOQ): comparison of the empirical and the statistical methods exemplified with GC-MS assays of abused drugs. Clin. Chem, 1994. 40(7): p. 1233-1238.

204. Karch, S.B., Karch's Pathology of Drug Abuse. 2002, Boca Raton, FL: CRC Press.

205. Dixon, S.D., Effects of transplacental exposure to cocaine and methamphetamine on the neonate. West J Med, 1989. 150(4): p. 436-442.

206. Ebrahim, S.H. and J. Gfroerer, Pregnancy-related substance use in the United States during 1996-1998. Obstet Gynecol Clin North Am, 2003. 101(2): p. 374-379.

207. Ostrea, E.M., A. Romero, and H. Yee, Adaption of the meconium drug test for mass screening. The Journal of Pediatrics, 1993. 122(1): p. 152-154.

208. Rogers, C., J. Hall, and J. Muto, Findings in newborns of cocaine-abusing mothers. Journal of Forensic Sciences, 1991. 36(4): p. 1074-1078.

209. Ross, D.L. and T.C. Chan, Sudden Deaths in Custody. 2006, Totowa, NJ: Humana Press.

210. Di Maio, T.G. and V.J.M. Di Maio, Excited Delirium Syndrome Cause of Death and Prevention. 2006, Boca Raton: Taylor \& Francis Group.

211. Ripple, M.G., et al., Detection of Cocaine and its Metabolites in Human Amniotic Fluid. Journal of Analytical Toxicology, 1992. 16(5): p. 328-331.

212. Sandberg, J.A. and G.D. Olsen, Cocaine and metabolite concentrations in the fetal guinea pig after chronic maternal cocaine administration. J Pharmacol Exp Ther, 1992. 260(2): p. 587-591. 
213. Callahan, C., et al., Measurement of gestational cocaine exposure: sensitivity of infants' hair, meconium, and urine. J Pediatr, 1992. 120(5): p. 763-768.

214. Clark, G.D., et al., The analysis of cocaine and benzoylecgonine in meconium. J Anal Toxicol, 1992. 16(4): p. 261-263.

215. Murphey, L.J., G.D. Olsen, and R.J. Konkol, Quantitation of benzoylnorecgonine and other cocaine metabolites in meconium by high-performance liquid chromatography. J Chromatogr B Analyt Technol Biomed Life Sci, 1993. 613(2): p. 330-335.

216. Xia, Y., et al., An LC-MS-MS method for the comprehensive analysis of cocaine and cocaine metabolites in meconium. Anal Chem, 2000. 72(4): p. 764-771.

217. Pichini, S., et al., Development and validation of a liquid chromatography-mass spectrometry assay for the determination of opiates and cocaine in meconium. $\mathrm{J}$ Chromatogr B Analyt Technol Biomed Life Sci, 2003. 794(2): p. 281-292.

218. Pichini, S., et al., Application of a validated high-performance liquid chromatographymass spectrometry assay to the analysis of $m$ - and p-hydroxybenzoylecgonine in meconium. J Chromatogr B Analyt Technol Biomed Life Sci, 2005. 820(1): p. 151-156.

219. Wang, P. and M.G. Bartlett, Collision-induced Dissociation Mass Spectra of Cocaine, and its Metabolites and Pyrolysis

Products. Journal of mass spectrometry, 1998. 33(10): p. 961-967.

220. Drummer, O.H., The forensic pharmacology of drugs of abuse. 2001, London: Arnold.

221. Martin, W.R., et al., The effects of morphine- and nalorphine- like drugs in the nondependent and morphine-dependent chronic spinal dog. J. Pharmacol. Exp. Ther. , 1976. 197: p. 517-532.

222. Lord, J.A.H., et al., Endogenous opioid peptides: multiple agonists and receptors. Nature, 1977. 267(5611): p. 495-499.

223. Di Chiara, G. and R.A. North, Neurobiology of opiate abuse. Trends in Pharmacol Sci, 1992. 13(5): p. 185-193.

224. Kelly, H.W., Drug-induced pulmonary dieseases, in Pharmacotherapy: A pathophysiological approach. 1993, Norwalk: Appleton \& Lange. p. 482-493.

225. DeBoer, L., The opiates and their derivatives. Texas Medicine, 1973. 69: p. 47-53.

226. Serturner, F.W.A., J. Pharm. F. Arzte. Apoth. Chem., 1806. 14(47-93): p. 47.

227. Forti, R.J., Opiate overdose. Pediatr. Rev, 2008. 28: p. 35-36.

228. Anderson, R.N., et al., Deaths: Injuries, 2001. . National Vital Statistics Reports. Centers for Disease Control and Prevention, US Dept of Health and Human Services, 2004. 52(21): p. 1-87.

229. Minino, A.M., et al., Deaths: Injuries, 2002. National Vital Statistics Reports. Centers for Disease Control and Prevention, US Dept of Health and Human Services, 2006. 54(10): p. 1-125.

230. Drummer, O.H., Recent trends in narcotic deaths. Ther Drug Monit, 2005. 27(6): p. 738740.

231. Teoh, W.H., F. Ithnin, and A.T. Sia, Comparison of an equal-dose spinal anesthetic for cesarean section and for post partum tubal ligation. Int J Obstet Anesth, 2008. 17(3): p. 228-232.

232. Ducarme, G., et al., Management of necrotizing pancreatitis in the third trimester of pregnancy. Arch Gynecol Obstet, 2008.

233. Carvalho, B., Respiratory depression after neuraxial opioids in the obstetric setting. Anesth Analg, 2008. 107(3): p. 956-961. 
234. Joranson, D.E., et al., Trends in medical use and abuse of opioid analgesics. JAMA, 2008. 283(13): p. 1710-1714.

235. Wisniewski, A.M., C.H. Purdy, and R.D. Blondell, The epidemiologic association between opioid prescribing, non-medical use, and emergency department visits. J Addict Dis, 2008. 27(1): p. 1-11.

236. Cone, E.J., et al., Evidence of morphine metabolism to hydromorphone in pain patients chronically treated with morphine. Journal of Analytical Toxicology, 2006. 30: p. 1-5.

237. Cone, E.J., et al., Evidence that morphine is metabolized to hydromorphone but not to oxymorphone. Journal of Analytical Toxicology, 2008. 32: p. 319-323.

238. Verispan, V. Top 200 generic drugs by units in 2005. Drug Topics 2006 [cited 2008 Septmber16];Available

from:http://www.drugtopics.com/drugtopics/data/articlestandard/drugtopics/102006/3112 93/article.pdf.

239. Baselt, R.C., The disposition of toxic drugs and chemicals in man. 8th ed. 2008, Foster City: Biomedical Publications.

240. Wallage, H.R. and J.P. Palmentier, Hydromorphone-related fatalities in ontario. J Anal Toxicol, 2006. 30(3): p. 202-209.

241. ElSohly, M.A., S. Feng, and T.P. Murphy, Analysis of Methadone and its Metabolites in Meconium by Enzyme Immunoassay (EMIT) and GC-MS. Journal of Analytical Toxicology, 2001. 25(1): p. 40-44.

242. Le, N.L., et al., The Detection of Oxycodone in Meconium Specimens. Journal of Analytical Toxicology, 2005. 29(1): p. 54-57.

243. ElSohly, M.A., et al., Immunoassay and GC-MS Procedures for the Analysis of Drugs of Abuse in Meconium. Journal of Analytical Toxicology, 1999. 23(6): p. 436-445.

244. Zhengxiang, Z., et al., Fragmentation pathways of heroin-related alkaloids revealed by ion trap and quadrupole time-of-flight tandem mass spectrometry. Rapid Commun. Mass Spectrom, 2008. 22(18): p. 2851-2862.

245. Plessinger, M., Prenatal exposure to amphetamines. Risks and adverse outcomes in pregnancy. Obstet Gynecol Clin North Am, 1998. 25(1): p. 119-138.

246. Burchfield, D., et al., Disposition and pharmacodynamics of methamphetamine in pregnant sheep. JAMA, 1991. 265(15): p. 1968-1973.

247. Acuff-Smith, K., et al., Preliminary evidence for methamphetamine-induced behavioral and ocular effects in rat offspring following exposure during early organogenesis. Psychopharmacology, 1992. 109(3): p. 255-263.

248. Yamamoto, Y., et al., Teratogenic effects of methamphetamine in mice. Nihon Hoigaku Zasshi, 1992. 46(2): p. 126-131.

249. Weissman, A. and S. Caldecott-Hazard, Developmental neurotoxicity to methamphetamines. Clin Exp Pharmacol Physiol, 1995. 22(5): p. 372-374.

250. Cho, D., et al., Behavioral teratogenicity of methamphetamine. J Toxicol Sci, 1991. 16(1): p. 37-49.

251. Arria, A., et al., Methamphetamine and other substance use during pregnancy: preliminary estimates from the Infant Development, Environment, and Lifestyle (IDEAL) study. Matern Child Health J, 2006. 10(3): p. 293-302.

252. Smith, L., et al., The infant development, environment, and lifestyle study: effects of prenatal methamphetamine exposure, polydrug exposure, and poverty on intrauterine growth. Pediatrics, 2006. 118(3): p. 1149-1156. 
253. Arria, A.M., et al., Methamphetamine and other substance use during pregnancy: preliminary estimates from the Infant Development, Environment, and Lifestyle (IDEAL) study. Matern Child Health J, 2006. 10(3): p. 293-302.

254. Smith, L.M., et al., The infant development, environment, and lifestyle study: effects of prenatal methamphetamine exposure, polydrug exposure, and poverty on intrauterine growth. Pediatrics, 2006. 118(3): p. 1149-1156.

255. Moore, C., et al., The detection of hydrocodone in meconium: two case studies. Journal of Analytical Toxicology, 1995. 19(6): p. 514-518.

256. Moore, C., A. Negrusz, and D. Lewis, Determination of drugs of abuse in meconium. J Chromatogr B Biomed Sci Appl, 1998. 713(1): p. 137-146.

257. Ryan, R., et al., Meconium analysis for improved identification of infants exposed to cocaine in utero. J Pediatr, 1994. 125(3): p. 435-440.

258. Gourley, G., B. Kreamer, and R. Arend, Excremental studies in human neonates. Identification of zinc coproporphyrin as a marker for meconium. Gastroenterology, 1990. 99(6): p. 1705-1709.

259. Moriya, F., et al., Detection of drugs-of-abuse in meconium of a stillborn baby and in stool of a deceased 41-day-old infant. Journal of Forensic Science, 1995. 40(3): p. 505508.

260. Ostrea, E.M., et al., Spectrum of gestational exposure to illicit drugs and other xenobiotic agents in newborn infants by meconium analysis. J Pediatr, 1998. 133(4): p. 513-515.

261. Apollonio, L., et al., Product ion mass spectra of amphetamine-type substances, designer analogues, and ketamine using ultra-performance liquid chromatography/tandem mass spectrometry. Rapid Commun. Mass Spectrom., 2006. 20(15): p. 2259-2264.

262. Cooks, R.G., et al., Ambient Mass Spectrometry. Science, 2006. 311: p. 1566-1570.

263. Takats, Z., et al., Mass spectometry sampling under ambient conditions with desorption electrospray ionization. Science, 2004. 306: p. 471-473.

264. Takats, Z., J.M. Wiseman, and R.G. Cooks, Ambient mass spectrometry using desporption electrospray ionization (DESI): instrumentation, mechanisms and applications in forensics, chemistry, and biology. Journal of mass spectrometry, 2005. 40: p. 1261-1275.

265. Karas, M. and F. Hillenkamp, laser desorption ionization of proteins with molecular masses exceeding 10000 daltons. Anal. Chem, 1988. 60: p. 2299-2301.

266. Fenn, J.B., et al., electrospray ionization for mass-spectrometry of large biomolecules. Science, 1989. 246: p. 64.

267. Smith, R.D., et al., New developments in biochemical mass spectrometry: Electrospray ionization. Anal. Chem, 1990. 62: p. 882-899.

268. Karas, M., D. Bachmann, and F. Hillenkamp, Influence of the wavelength in highirradiance ultraviolet laser desorption mass spectrometry of organic molecules. Anal. Chem, 1985. 57: p. 2935-2939.

269. Zaluzec, E.J., D.A. Gage, and J.T. Watson, matrix-assisted laser desorption ionization mass spectrometry: applications in peptide and protein characterization. Protein expression and purification, 1995. 6: p. 109-123.

270. Costello, C.E., Time, life and mass spectrometry. Biophysical Chemistry, 1997. 68(1-3): p. 173-188.

271. Gates, P. Matrix assisted laser desorption ionization (MALDI). 2004 [cited 2008 07/22/2008]. 
272. Dreisewerd, K., The desorption process in MALDI. Chem. Rev, 2003. 103: p. 395-425.

273. Knochenmuss, R., Ion formation mechanisms in UV-MALDI. Analyst, 2006. 131: p. 966986.

274. Fitzgerald, M.C. and L.M. Smith, mass spectrometry of nucleic acids: the promise of matrix-assisted laser desorption-ionization (MALDI) mass spectrometry. Annu. Rev. Biophys. Biomol. Struct., 1995. 24: p. 117-140.

275. Moore, C., D. Lewis, and J. Leikin, False-positive and false-negative rates in meconium drug testing. Clin Chem, 1995. 41(11): p. 1614-1616.

276. Thompson, R., Identification of drugs, drug metabolites, and other compounds in urine by permethylation and gas-phase analysis. Res Commun Chem Pathol Pharmacol, 1977. 16(1): p. 145-154.

277. Wilson, J.M., L.J. Williamson, and V.A. Raisys, Simultaneous measurement of secondary and tertiary tricyclic antidepressants by GC/MS chemical ionization mass fragmentography. Clin Chem, 1977. 23(6): p. 1012-1017.

278. Dugal, R., et al., An integrated methodological approach to the computer-assisted gas chromatographic screening of basic drugs in biological fluids using nitrogen selective detection. J Anal Toxicol, 1980. 4(1): p. 1-12.

279. Brotherton, H.O. and R.A. Yost, Rapid screening and confirmation for drugs and metabolites in racing animals by tandem mass spectrometry. Am J Vet Res, 1984. 45(11): p. 2436-2440.

280. Straub, K.M., P. Rudewicz, and C. Garvie, 'Metabolic mapping' of drugs: rapid screening techniques for xenobiotic metabolites with m.s./m.s. techniques. Xenobiotica, 1987. 17(3): p. 413-422.

281. Jones, A.D., et al., Analysis of mercapturic acid conjugates of xenobiotic compounds using negative ionization and tandem mass spectrometry. Biol Mass Spectrom, 1993. 22(1): p. 68-76.

282. Brzezinka, H., P. Bold, and H. Budzikiewicz, A screening method for the rapid detection of barbiturates in serum by means of tandem mass spectrometry. Biol Mass Spectrom, 1993. 22(6): p. 346-350.

283. Weinmann, W. and M. Svoboda, Fast screening for drugs of abuse by solid-phase extraction combined with flow-injection ionspray-tandem mass spectrometry. J Anal Toxicol, 1998. 22(4): p. 319-328.

284. Nordgren, H.K. and O. Beck, Direct screening of urine for MDMA and MDA by liquid chromatography-tandem mass spectrometry. J Anal Toxicol, 2003. 27(1): p. 15-19.

285. Eichhorst, J., et al., Urinary screening for methylphenidate (Ritalin) abuse: a comparison of liquid chromatography-tandem mass spectrometry, gas chromatography-mass spectrometry, and immunoassay methods. Clin Biochem Rev, 2004. 37(3): p. 175-183.

286. Nordgren, H.K., et al., Application of direct urine LC-MS-MS analysis for screening of novel substances in drug abusers. J Anal Toxicol, 2005. 29(4): p. 234-239.

287. Allen, K.R., et al., Replacement of immunoassay by LC tandem mass spectrometry for the routine measurement of drugs of abuse in oral fluid. Ann Clin Biochem, 2005. 42(Pt 4): p. $277-284$.

288. Allen, K.R., Interference by venlafaxine ingestion in the detection of tramadol by liquid chromatography linked to tandem mass spectrometry for the screening of illicit drugs in human urine. Clin Toxicol (Phila). 2006. 44(2): p. 147-153. 
289. Irving, R.C. and S.J. Dickson, The detection of sedatives in hair and nail samples using tandem LC-MS-MS. Forensic Sci Int, 2007. 166(1): p. 58-67.

290. Øiestad, E.L., U. Johansen, and A.S. Christophersen, Drug screening of preserved oral fluid by liquid chromatography-tandem mass spectrometry. Clin Chem, 2007. 53(2): p. 300-309.

291. Björnstad, K., A. Helander, and O. Beck, Development and clinical application of an LCMS-MS method for mescaline in urine. J Anal Toxicol, 2008. 32(3): p. 227-231.

292. Gergov, M., et al., Toxicological screening of urine for drugs by liquid chromatography/time-of-flight mass spectrometry with automated target library search based on elemental formulas. Rapid Commun Mass Spectrom, 2001. 15(8): p. 521-526.

293. Pelander, A., et al., Toxicological screening with formula-based metabolite identification by liquid chromatography/time-of-flight mass spectrometry. Anal Chem, 2003. 75(21): p. 5710-5718.

294. Ojanperä, L., et al., Application of accurate mass measurement to urine drug screening. $\mathrm{J}$ Anal Toxicol, 2005. 29(1): p. 34-40.

295. Venter, A., P.E. Sojka, and R.G. Cooks, Droplet dynamics and ionization mechanisms in desorption electrospray ionization mass spectrometry. Anal Chem, 2006. 78: p. 85498555.

296. Ifa, D.R., et al., Forensic analysis of inks by imaging desorption electrospray ionization (DESI) mass spectrometry. Analyst, 2007. 132: p. 461-467.

297. Costa, A.B. and R.G. Cooks, Simulation of atmospheric transport and droplet-thin film collisions in desorption electrospray ionization. Chem. Commun, 2007: p. 3915-3917.

298. Manicke, N.E., et al., Desorption electrospray ionization (DESI) mass spectrometry and tandem mass spectrometry (MS/MS) of phospholipids and sphingolipids: ionization, adduct formation, and fragmentation. J Am Soc Mass Spectrom, 2008. 19(4): p. 531-543. 